\title{
DEVELOPMENT AND GROUND TESTING OF DIRECT MEASURING SKIN FRICTION GAGES FOR HIGH ENTHALPY SUPERSONIC FLIGHT TESTS
}

\author{
by \\ Theodore Brooke Smith \\ Dissertation submitted to the Faculty of the \\ Virginia Polytechnic Institute and State University \\ In partial fulfillment of the requirements for the degree of
}

Doctor of Philosophy

in

Aerospace Engineering

\author{
APPROVED: \\ Dr. Joseph A. Schetz, Chairman \\ Dr. Bernard Grossman \\ Dr. Roger Simpson \\ Dr. William Devenport \\ Dr. Trong Bui
}

September 10, 2001

Blacksburg, VA

Keywords: skin friction, scramjet, hypersonic, Hyper-X, X-43A

Copyright 2001 


\title{
DEVELOPMENT AND GROUND TESTING OF DIRECT MEASURING SKIN FRICTION GAGES FOR HIGH ENTHALPY SUPERSONIC FLIGHT TESTS
}

\author{
Theodore B. Smith \\ Dr. Joseph Schetz, Chairman \\ Aerospace Engineering
}

\begin{abstract}
(ABSTRACT)
A series of direct-measuring skin friction gages were developed for a high-speed, high-temperature environment of the turbulent boundary layer in flows such as that in supersonic combustion ramjet (scramjet) engines, with a progression from free-jet ground tests to a design for an actual hypersonic scramjet-integrated flight vehicle. The designs were non-nulling, with a sensing head that was flush with the model wall and surrounded by a small gap. Thus, the shear force due to the flow along the wall deflects the head, inducing a measurable strain. Strain gages were used to detect the strain. The gages were statically calibrated using a direct force method. The designs were verified by testing in a well-documented Mach 2.4 cold flow. Results of the cold-flow tests were repeatable and within $15 \%$ of the value of $\mathrm{C}_{\mathrm{f}}$ estimated from simple theory. The first gage design incorporated a cantilever beam with semiconductor strain gages to sense the shear on the floating head. Cooling water was routed both internally and around the external housing in order to control the temperature of the strain gages. This first gage was installed and tested in a rocket-based-combined-cycle (RBCC) engine model operating in the scramjet mode. The free-jet facility provided a Mach 6.4 flow with $\mathrm{P}_{0}=$ 1350 psia $(9310 \mathrm{kPa})$ and $\mathrm{T}_{0}=2800^{\circ} \mathrm{R}\left(1555^{\circ} \mathrm{K}\right)$. Local wall temperatures were measured between 850 and $900^{\circ} \mathrm{R}\left(472-500^{\circ} \mathrm{K}\right)$. Output from the RBCC scramjet tests was reasonable and repeatable. A second skin friction gage was designed for and tested in a wind tunnel model of the Hyper-X flight vehicle scramjet engine. These unsuccessful tests revealed the need for a radically different skin friction gage design. The third and final skin friction gage was specifically developed to be installed on the Hyper-X flight vehicle. Rather than the cantilever beam and semiconductor strain gages, the third skin friction gage made use of a flexure ring and metal foil strain gages to sense
\end{abstract}


the shear. The water-cooling and oil-fill used on the previous skin friction sensors were eliminated. It was qualified for flight through a rigorous series of environmental tests, including pressure, temperature, vibration, and heat flux tests. Finally, the third skin friction gage was tested in the Hyper-X Engine Model (HXEM), a full-scale-partial-width wind tunnel model of the flight vehicle engine. These tests were conducted at Mach 6.5 enthalpy with $\mathrm{P}_{0}=555 \mathrm{psia}(3827 \mathrm{kPa})$ and $\mathrm{h}_{0}=900 \mathrm{Btu} / \mathrm{lb}_{\mathrm{m}}$ in a freejet facility. The successful testing in the wind tunnel scramjet model provided the final verification of the gage before installation in the flight vehicle engine. The development, testing, and results of all three skin friction gages are discussed. 


\section{ACKNOWLEDGEMENTS}

First and foremost, this work could not have been completed but by the grace of God and His Son, Jesus Christ. It is to God that I humbly give my greatest thanks for the finishing of this work, and beyond that, for my very life, here and hereafter.

God, in his providence, placed me under the advisement of Dr. Joseph A. Schetz, to whom I am in debt of gratitude. His knowledge, experience, wisdom, and dedication have been a constant, underlying all of the work done on this project. I also thank Dr. B. Grossman, Dr. R. Simpson, Dr. W. Devenport, and Dr. T. Bui for their service on my committee and the knowledge that they have imparted to me in and out of the classroom.

I would also like to recognize my wife, Julie, who has given faithful encouragement at every step of the graduate school process. I truly could not have finished this document without her diligent help every day.

My friends and fellow graduate students also deserve credit for sharing in trials, and providing support and comic relief. Among them, Andy Ko will be remembered for his dependable friendship throughout our time at Virginia Tech. Ross Stilling, Alex Sang, Matt Maclean, Samantha Magill, and Wade Pulliam were all members of the skin friction research group, and each helped out and added their own insights to the project at different times. The manufacturing of the gages, and the application and wiring of strain gages, along with other assorted wiring and machining jobs, were skillfully accomplished by the machine shop workers, Bruce Stanger, Greg Dudding, Mike Vaught, and Kent Morris, and our electrician, Gary Stafford.

Special thanks go to Karen Cabell of NASA Langley's Arc-Heated Scramjet Test Facility. Her accommodation of our schedules and hardware made the testing in the scramjet engine at NASA possible.

This research was supported by the National Aeronautics and Space Administration out of the Dryden Flight Research Center, with Dr. Trong Bui serving as contract monitor. 


\section{TABLE OF CONTENTS}

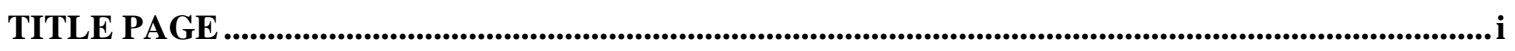

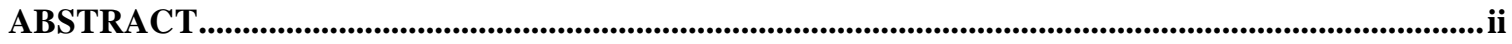

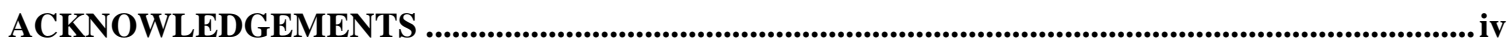

TABLE OF CONTENTS ........................................................................................................................................ v

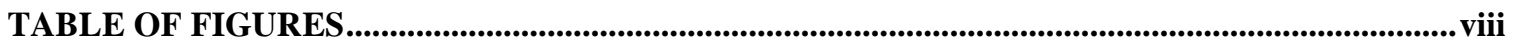

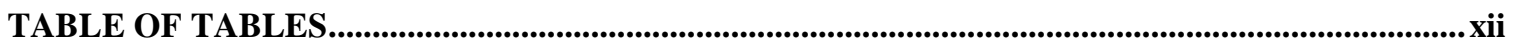

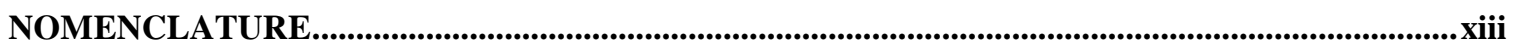

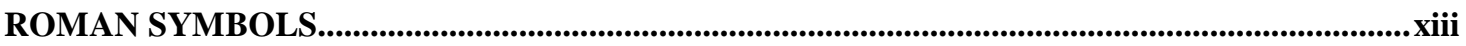

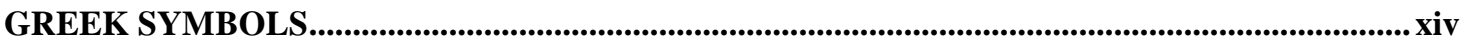

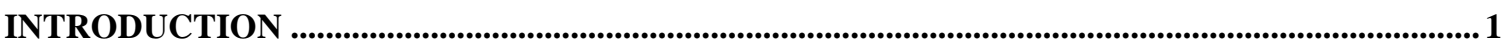

1.1 Importance of Measuring Skin Friction................................................................................................... 1

1.2 Specific Motivation: The Hyper-X ........................................................................................................ 3

1.3 Measurement Methods ................................................................................................................................... 4

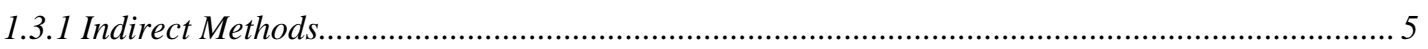

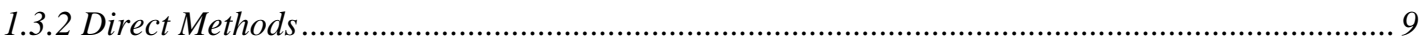

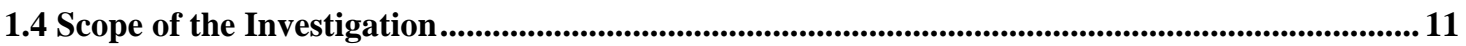

DESIGN OVERVIEW AND CONSTRAINTS .......................................................................................... 14

2.1 General Design Attributes.................................................................................................................. 14

2.2 Floating Head Design Attributes and Concerns ..................................................................................... 17

2.3 Strain Gage Theory and Practice ..........................................................................................................20

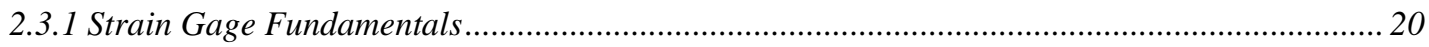

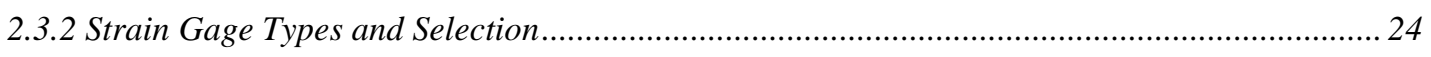

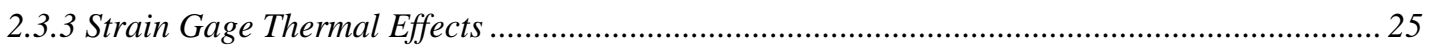

TEST APPARATUS AND INSTRUMENTATION ......................................................................................28

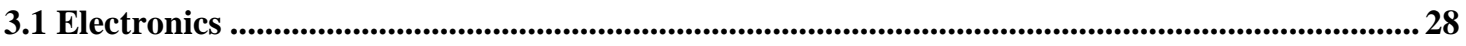

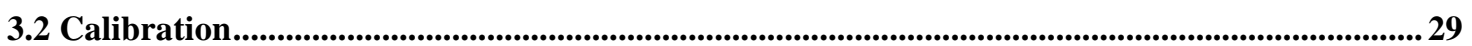

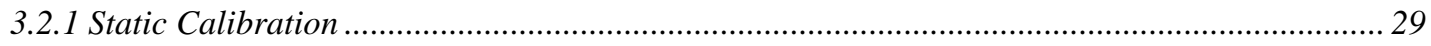

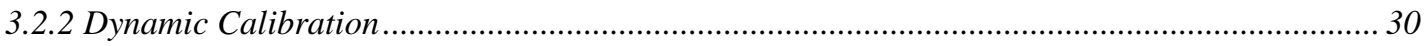

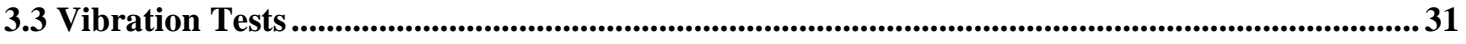

3.4 Virginia Tech 9-inch x 9-inch Supersonic Wind Tunnel ................................................................33

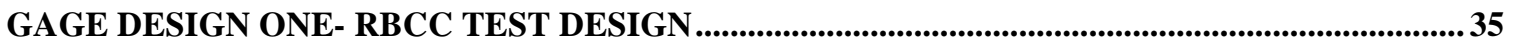

4.1 Gage Design ....................................................................................................................................................... 35 


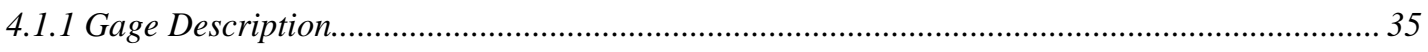

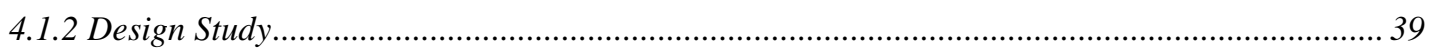

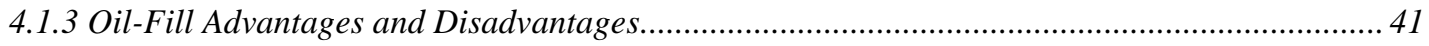

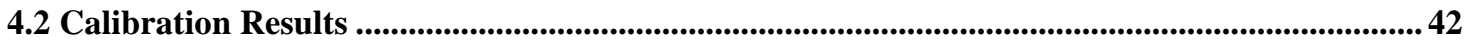

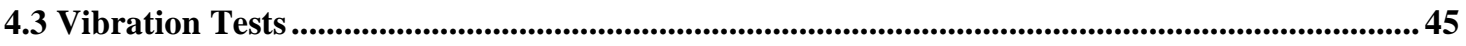

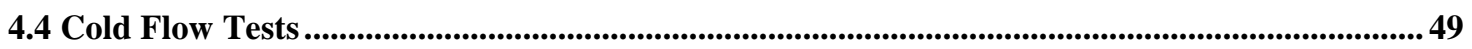

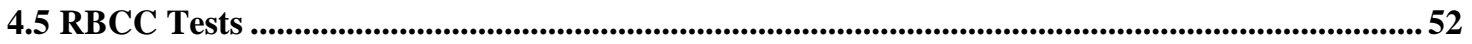

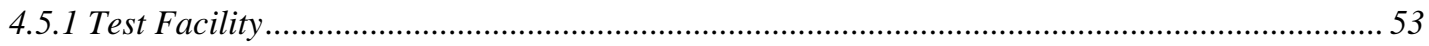

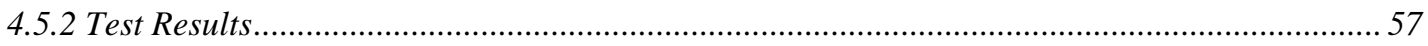

GAGE DESIGN TWO- HXEM TEST DESIGN............................................................................62

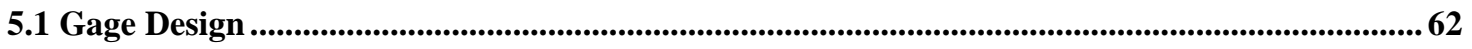

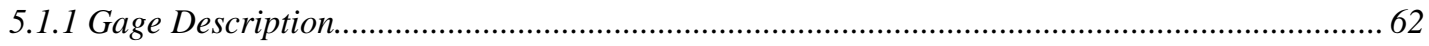

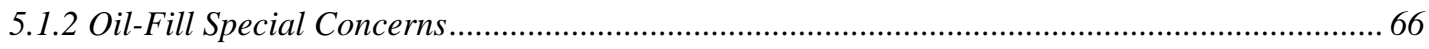

5.2 Calibration Results ..........................................................................................................................667

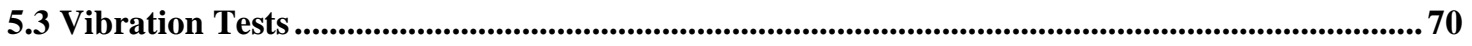

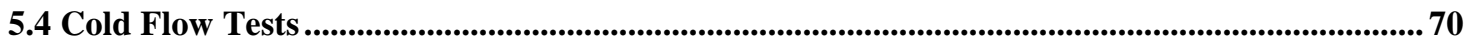

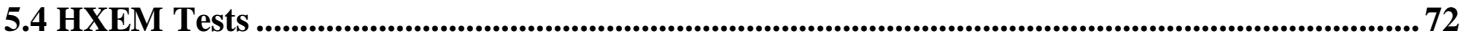

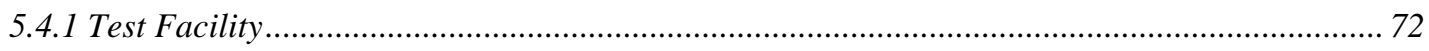

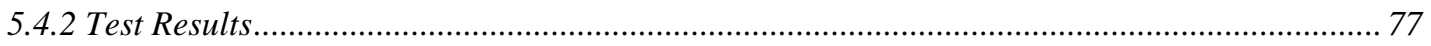

5.5 Concluding Remarks Concerning Gage Design Two................................................................... 79

GAGE DESIGN THREE- HYPER-X FLIGHT VEHICLE DESIGN ..............................................8 81

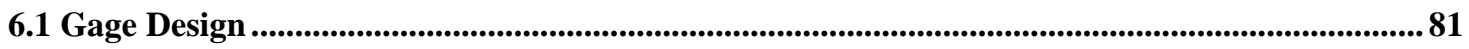

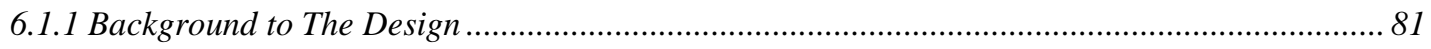

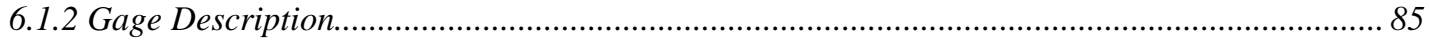

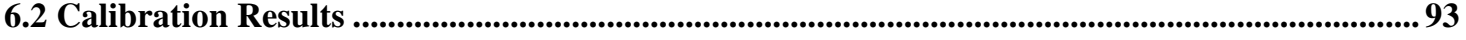

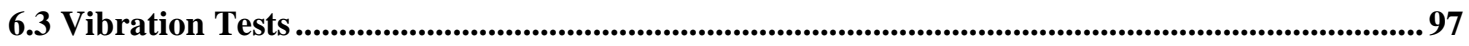

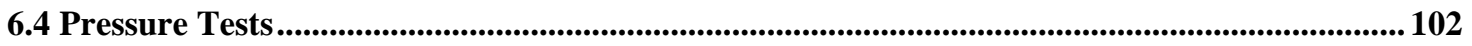

6.5 Temperature Tests ............................................................................................................... 103

6.6 Heat Load Tests......................................................................................................................107

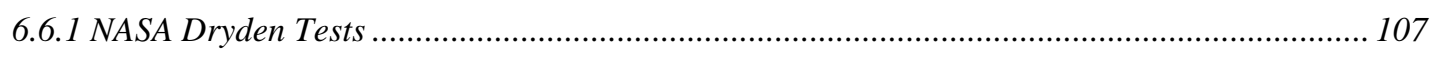

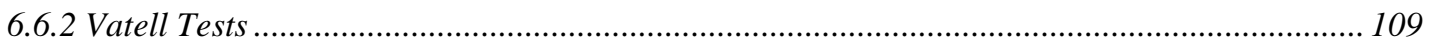

6.7 Cold Flow Tests ............................................................................................................................ 115

6.8 HXEM Tests ............................................................................................................................... 119

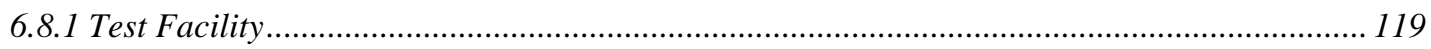

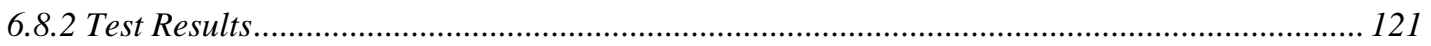

6.9 Concluding Remarks About Gage Design Three .......................................................................... 127

HEAT FLUX GAGE DESIGN FOR HYPER-X FLIGHT VEHICLE ................................................. 129 


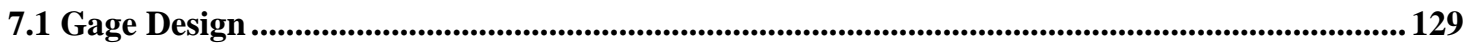

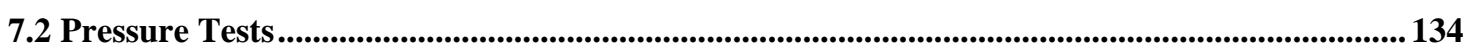

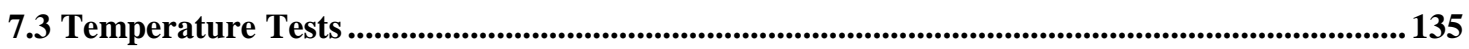

7.4 Heat Load Tests................................................................................................................................................ 137

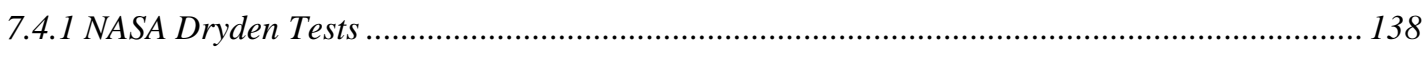

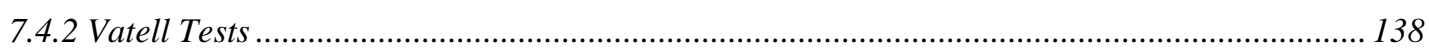

7.5 HXEM Tests ................................................................................................................................................... 143

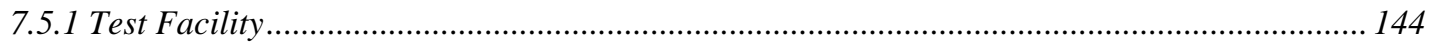

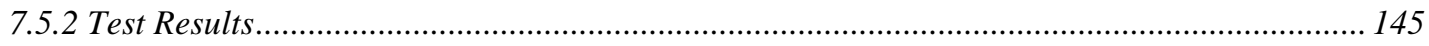

7.6 Concluding Remarks About The Heat Flux Gage ............................................................................... 154

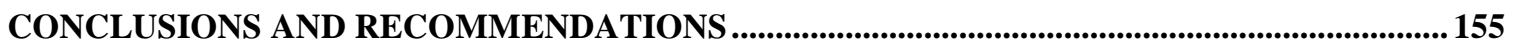

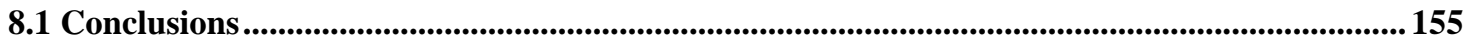

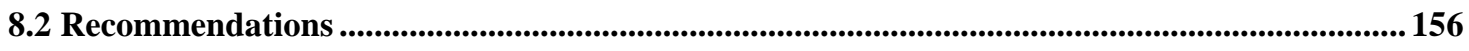

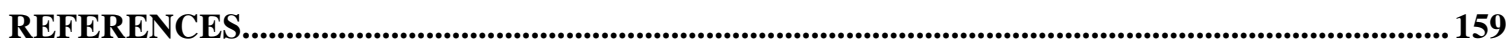

APPENDIX A: UNCERTAINTY ANALYSIS ................................................163

A.1 Calibration and Repeatability .................................................................163

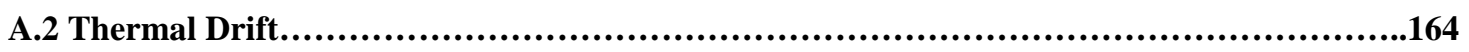

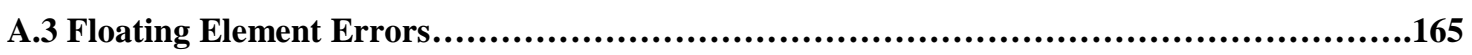

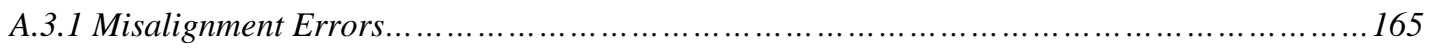

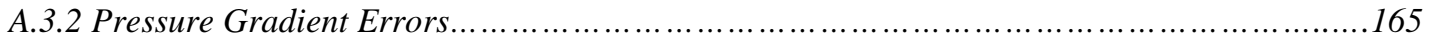

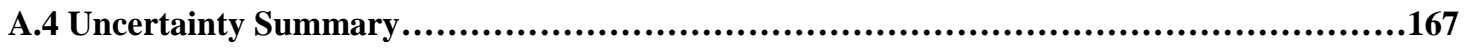

APPENDIX B: FORTRAN CODE FOR HEAT FLUX GAGE POST-PROCESSING...............168

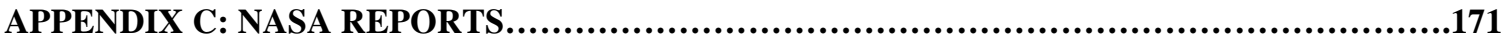

C.1 Calibration Test Report...................................................................172

C.2 Qualification Test Report...............................................................186

C.3 Handling and Cleanliness Report......................................................193

C.4 NASA Langley HXEM/AHSTF Skin Friction and Heat Flux Sensor Test Report..............200

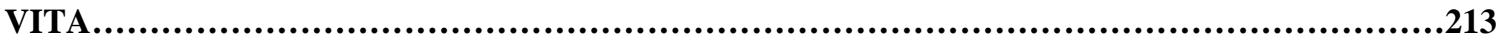




\section{TABLE OF FIGURES}

Figure 1: Artist's Sketch of Hyper-X Flight Vehicle...............................................................................4

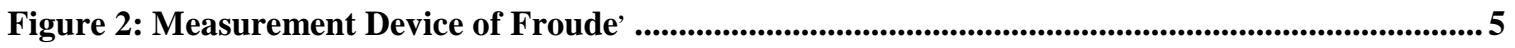

Figure 3: Indirect Skin Friction Measurement Techniques .................................................................6

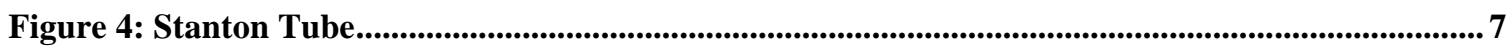

Figure 5: Dhawan's Skin Friction Balance ............................................................................... 10

Figure 6: Example of Nulling Skin Friction Gage of Kistler (from Paros) ......................................11

Figure 7: Acceleration Insensitive Skin Friction Balance for Flight Tests (Lyons) ................................. 12

Figure 8: Skin Friction Gage General Features ..................................................................................... 14

Figure 9: Mounting Hole for Hyper-X Skin Friction Gages ...................................................................... 16

Figure 10: Typical Floating Head Configuration.................................................................................... 17

Figure 11: Floating Head Geometric Parameters ...................................................................................... 18

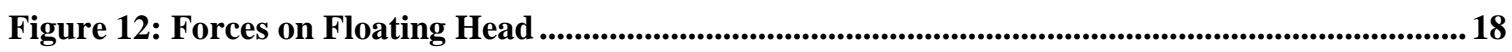

Figure 13: Wheatstone Bridge ......................................................................................................... 22

Figure 14: Static Calibration Method .......................................................................................................... 29

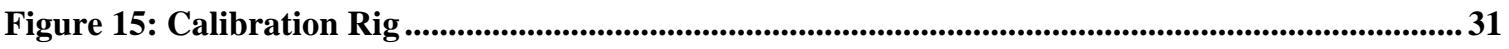

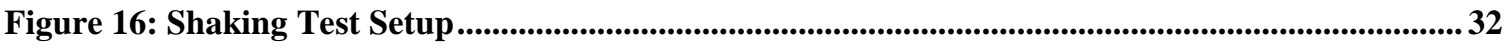

Figure 17: Virginia Tech 9-in. x 9-in. Supersonic Wind Tunnel a) Side View b) Top View................ 34

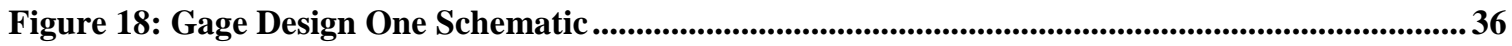

Figure 19: Design One Floating Element, Beam, Base, and Hypodermic Tubes ...............................38

Figure 20: Design One Completed Gage ............................................................................................................. 39

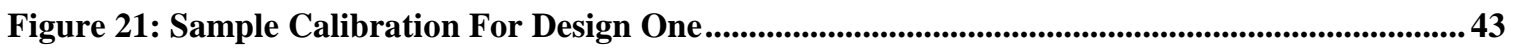

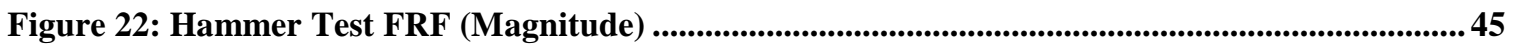

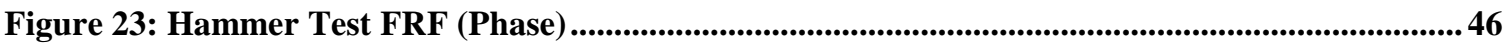

Figure 24: Shaking Test FRF (Magnitude)........................................................................................47

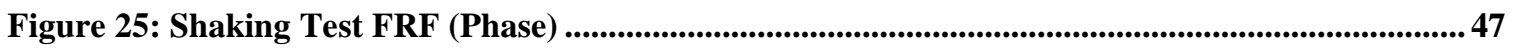

Figure 26: Shaking Tests Coherence .............................................................................................48

Figure 27: Shaking Tests Power Spectral Density ...............................................................................48

Figure 28: Supersonic Wind Tunnel Test Results: Run 3 ..................................................................50

Figure 29: Supersonic Wind Tunnel Test Results: Run4 ....................................................................50

Figure 30: GASL's Blowdown Test Facilities' ................................................................................53

Figure 31: GASL Leg IV Heating Systems .......................................................................................54

Figure 32: GASL Leg IV Test Section (not the same as used in RBCC tests) .......................................55

Figure 33: GASL Leg IV Operating Envelope .....................................................................................55

Figure 34: RBCC Engine Skin Friction Sensor Location ..........................................................................56

Figure 35: RBCC Test 21 ..............................................................................................................58

Figure 36: RBCC Test 22 ..........................................................................................................................5 59 


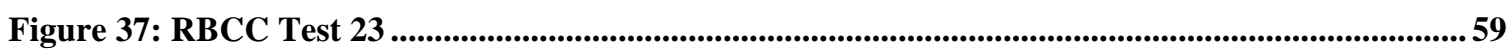

Figure 38: Gage Design Two Sketch ................................................................................................................63

Figure 39: Gage Design Two Internal Components.......................................................................................... 64

Figure 40: Design Two Housing and Internal Components ...............................................................................65

Figure 41: Three Assembled Gages (Design Two) ………….................................................................................65

Figure 42: Sample Static Calibration for Design Two.......................................................................................67

Figure 43: Dynamic Calibration Result- a) Raw Voltage b) Shear Level ................................................69

Figure 44: Supersonic Wind Tunnel Test Results: Run 3 ............................................................................. 71

Figure 45: Supersonic Wind Tunnel Test Results: Run 4 ............................................................................ 71

Figure 46: Schematic of NASA Langley AHSTF .............................................................................. 72

Figure 47: AHSTF Arc Heater and Plenum Chamber .........................................................................73

Figure 48: Flight Mach Number Simulation Technique .......................................................................... 74

Figure 49: AHSTF Operational Envelope ........................................................................................... 74

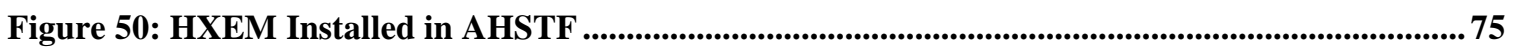

Figure 51: Wiring Diagram for Single Skin Friction Signal in AHSTF.................................................76

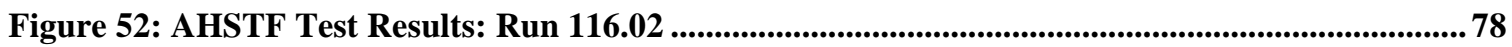

Figure 53: AHSTF Test Results: Run 117.01 ..................................................................................... 78

Figure 54: AHSTF Test Results: Run 117.01 Temperature ..............................................................79

Figure 55: Flexure Ring Concept and Nomenclature ........................................................................83

Figure 56: Sketch of a Flexure Ring-based Skin Friction Gage....................................................................... 84

Figure 57: Flexure Ring for Third Design .....................................................................................................85

Figure 58: Design Three Internal Components (Head, Adapter, Flexure Ring, Counterweight)......... 87

Figure 59: Design Three Internal Components..............................................................................87

Figure 60: Design Three Exploded View ...........................................................................................................90

Figure 61: Design Three Print ..................................................................................................................................91

Figure 62: Design Three Internal Components Mounted in "Housing b" .............................................92

Figure 63: Design Three Final Assembly ...........................................................................................93

Figure 64: Sample Calibration For Design Three...............................................................................................95

Figure 65: Angular Calibration Notation ..........................................................................................................96

Figure 66: Angular Calibration Output............................................................................................................96

Figure 67: Vibration Test Setup ...............................................................................................................97

Figure 68: Inspection of Broken Flexure Ring Constrained Within Housing ..........................................99

Figure 69: X-Axis FRF Magnitude................................................................................................................... 100

Figure 70: X-Axis FRF Phase ..................................................................................................................... 100

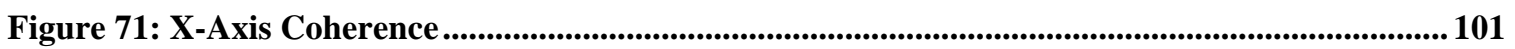

Figure 72: Pressure Environmental Qualification Test Results ...............................................................103

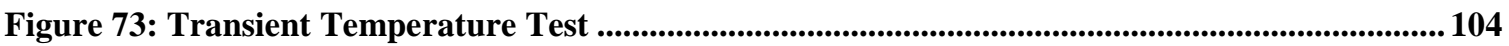


Figure 74: Steady State Temperature Test ..................................................................................................... 105

Figure 75: Single Temperature Response Profile............................................................................................... 106

Figure 76: Heat Load Test Setup Sketch ........................................................................................................... 108

Figure 77: Heat Load Test Setup: Skin Friction Gage Mounted in Copper Block and Coated with

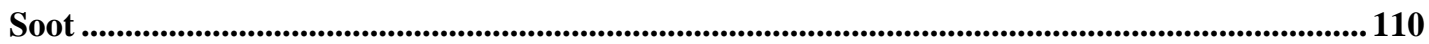

Figure 78: Heat Load Test Setup: a) Gage Withdrawn b) Gage Up Close to Heater Plate................. 111

Figure 79: Heat Load Test Setup: Shielded Gage During Heater Ramp-Up.........................................112

Figure 80: Heat Load Test Results: 30 Btu/ft ${ }^{2}$........................................................................................... 113

Figure 81: Heat Load Test Results: $70 \mathrm{Btu} / \mathrm{ft}^{2} \mathrm{~s}$.......................................................................114

Figure 82: Copper Insert for Supersonic Wind Tunnel Tests..............................................................116

Figure 83: Supersonic Wind Tunnel Test Results: Run 0 ........................................................................117

Figure 84: Supersonic Wind Tunnel Test Results: Run 3 .................................................................. 118

Figure 85: Modification for HXEM Tests.......................................................................................... 120

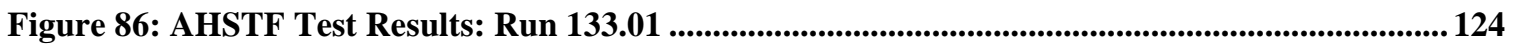

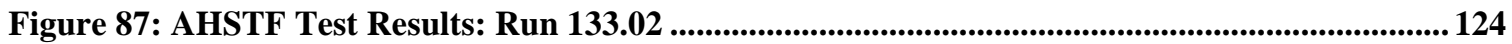

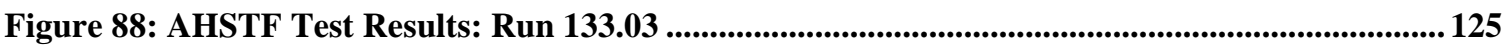

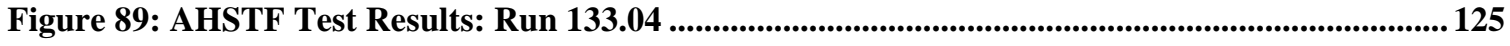

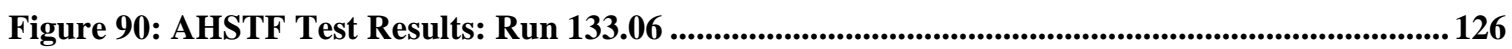

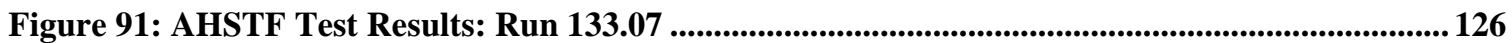

Figure 92: AHSTF Test Results: Gage Temperature History ..................................................................... 127

Figure 93: Heat Flux Copper Plug Sketch ........................................................................................................ 130

Figure 94: Coaxial Thermocouple Sketch (not to scale) ........................................................................130

Figure 95: Assembled Heat Flux Gage (Connectors Shown to Identify Thermocouples: Blue-Type T,

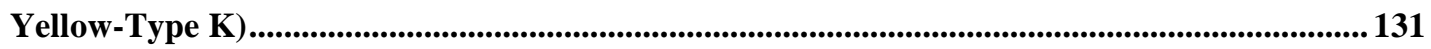

Figure 96: Wiring Schematic for Heat Flux Gage (From T. Bui).............................................................. 132

Figure 97: Pressure Environmental Qualification Test Results ............................................................... 135

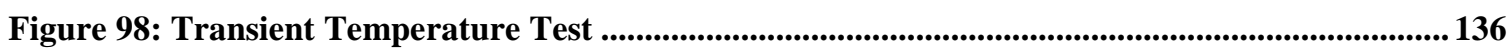

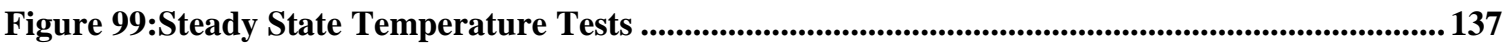

Figure 100: Heat Load Test Setup: Heat Flux Gage Mounted in Copper Block and Coated with Soot

Figure 101: Heat Load Test Setup: Shielded Gage During Heater Ramp-Up...................................... 140

Figure 102: Heat Load Test Results: 30 Btu/ft ${ }^{2} \mathrm{~s}$............................................................................................. 141

Figure 103: Heat Load Test Results: $70 \mathrm{Btu} / \mathrm{ft}^{2} \mathrm{~s}$......................................................................................... 142

Figure 104: Heat Load Test Results: 100 Btu/ft2s ................................................................................... 143

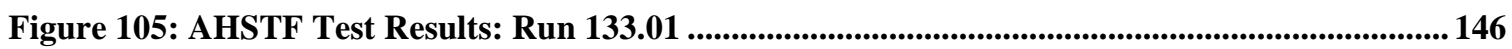

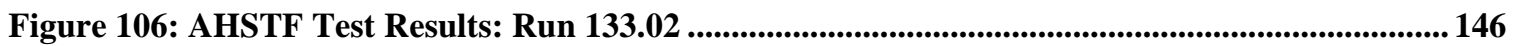

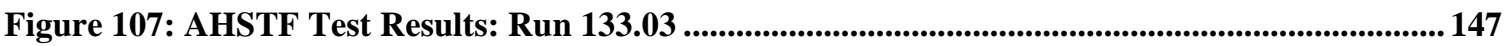


Figure 108: AHSTF Test Results: Run 133.04 .............................................................................................. 147

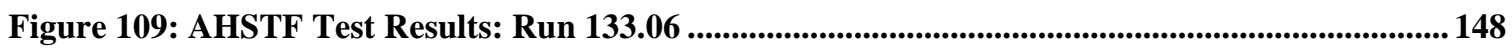

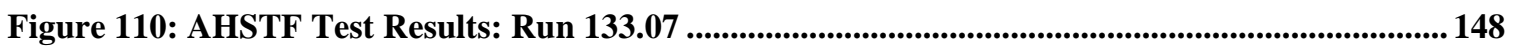

Figure 111: AHSTF Test Results: Gage Backside Temperature History ............................................... 149

Figure 112: AHSTF Heat Flux Estimate (Class 3): Run 133.01 ..................................................................151

Figure 113: AHSTF Heat Flux Estimate (Class 3): Run 133.02 ....................................................... 151

Figure 114: AHSTF Heat Flux Estimate (Class 3): Run 133.03 ........................................................152

Figure 115: AHSTF Heat Flux Estimate (Class 3): Run 133.04 .............................................................. 152

Figure 116: AHSTF Heat Flux Estimate (Class 3): Run 133.06 ............................................................... 153

Figure 117: AHSTF Heat Flux Estimate (Class 3): Run 133.07 .........................................................153 


\section{TABLE OF TABLES}

Table 1: Effect of Skin Friction on Specific Impulse of Scramjet Vehicle ................................................2

Table 2: Supersonic Wind Tunnel Resuults Summary ......................................................................................51

Table 3: RBCC Test Results Summary ...................................................................................................5

Table 4: Supersonic Wind Tunnel Results Summary ......................................................................116

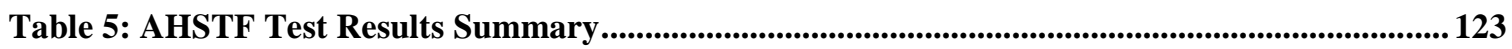

Table 6: AHSTF Test Results Summary ....................................................................................................149

Table 7: AHSTF Test Heat Flux Estimates ...................................................................................................150 


\section{NOMENCLATURE}

\section{ROMAN SYMBOLS}

$A_{\text {head }}$

$\mathrm{C}_{\mathrm{f} .} \ldots$

Floating Head Area

C.

c.

D

$\mathrm{D}$

$\mathrm{D}_{0}$

E.

$\mathrm{E}_{\mathrm{i}}$

$\mathrm{E}_{0}$

F.

G.

g.

GF

I. .......

If.

$\mathrm{k}$.

L.

$\mathrm{M}$

$\mathrm{m}$

P.

$\mathrm{P}_{0}$

Pr.

q.

$\mathrm{q}_{\mathrm{w}}$

$\mathrm{R}$

s.

St.

$\mathrm{T}$.

$\mathrm{T}_{0}$

TCGF.....

TCR

$\mathrm{U}_{\mathrm{e}}$

$\mathrm{u}^{*}$

$\mathrm{u}^{+}$

V.

$\mathrm{X}$.

$\mathrm{Y}$

$\mathrm{y}^{+}$

$\mathrm{Z}$

Skin Friction Coefficient

.Lip size, Specific Heat

Beam Width

Floating Element Diameter

.Internal Diameter of Beam

..Outer Diameter of Beam

..Modulus of Elasticity

..Excitation Voltage

.Output Voltage

Force

..Gap Size

Gravitational Acceleration Constant

Gage Factor

Area Moment of Inertia

Specific Impulse

Thermal Conductivity

.Length

.Mach Number, Moment

.Mass

.Point Load, Pressure ..Total Pressure ..Prandtl Number Dynamic Pressure ..Wall Heat Flux Resistance Side Length of a Square Stanton number Temperature

Total Temperature Temperature Coefficient of Gage Factor .Temperature Coefficient of Resistance Boundary Layer Edge Velocity .Friction Velocity Non-dimensional Velocity for Turbulent Boundary Layers Velocity Distance Deflection Non-dimensional Distance for Turbulent Boundary Layers Floating Head Protrusion 


\section{GREEK SYMBOLS}

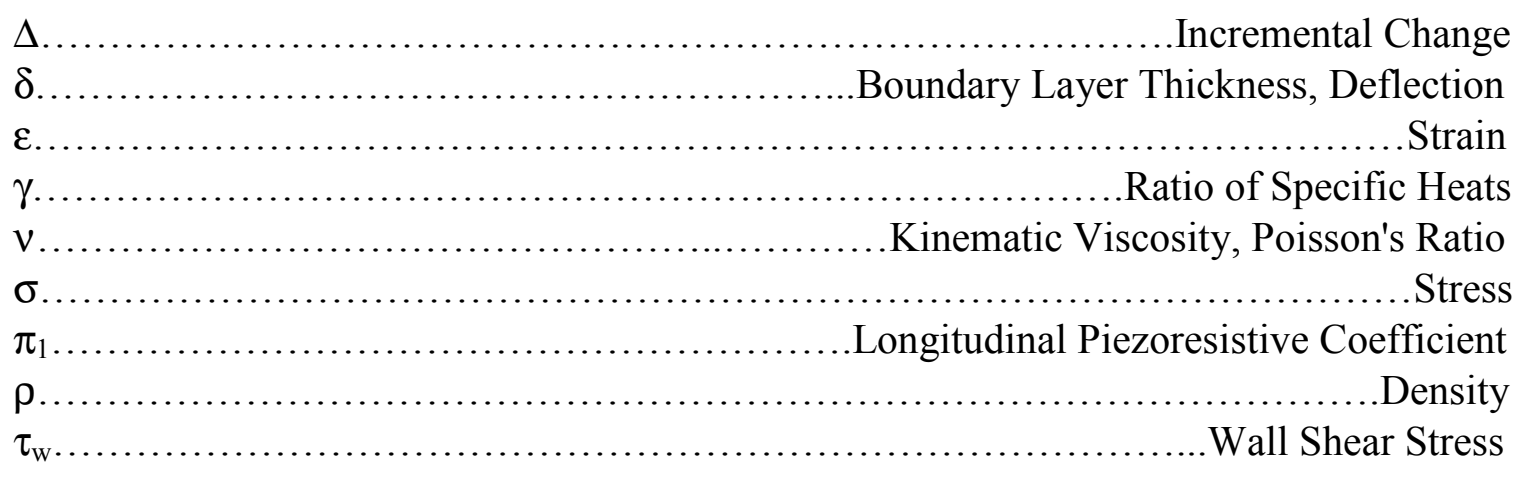




\section{CHAPTER ONE:}

\section{INTRODUCTION}

\subsection{Importance of Measuring Skin Friction}

The aerodynamic drag force on a body in a fluid flow can be separated into two components, pressure drag and friction drag. The pressure drag is the force necessary to accelerate the flow around the body, while the friction drag is caused by the viscous interaction between the fluid and the surface of the body ${ }^{1}$. The history of the prediction of pressure drag has resulted in mathematical and computational methods that achieve satisfactory accuracy in a wide variety of cases. Treating the viscous region near the surface of a body (the "boundary layer"), on the other hand, has required a much greater dependency on empirical data. Only the simplest cases, such as the laminar, incompressible, constant-property flow over a flat plate ${ }^{2}$, have been completely modeled analytically. It is very difficult to predict the behavior of complex, three-dimensional turbulent boundary layers, such as are often of practical interest to the engineer.

Therefore, measurements of skin friction have been vital to the understanding of complex flows and being able to evaluate and predict the performance of aerodynamic components $^{3}$. Scientifically, one of the fundamental correlating parameters of turbulent boundary layers is the friction velocity,

$$
u^{*}=\sqrt{\frac{\tau_{w}}{\rho}}=U_{e} \sqrt{\frac{C_{f}}{2}}
$$

\section{Equation 1}

in which the wall shear, $\tau_{\mathrm{w}}$, or its non-dimensional counterpart, the skin friction coefficient,

$$
C_{f}=\frac{\tau_{w}}{1 / 2 \rho U_{e}^{2}}
$$

\section{Equation 2}

appears, along with the fluid density, $\rho$, and the local boundary layer edge velocity, $U_{e}$. In turbulent boundary layers the friction velocity is used to non-dimensionalize velocity and distances, such as $\mathrm{u}^{+}=\mathrm{U} / \mathrm{u}^{*}$ and $\mathrm{y}^{+}=\mathrm{yu}^{*} / \mathrm{v}$ where $\mathrm{U}$ is the local tangential velocity, 
$\mathrm{y}$ is the distance from the surface, and $v$, is the kinematic viscosity of the fluid. These parameters are then used in defining the defect law and the law of the wall .

Many practical reasons for measuring or predicting skin friction abound. In highspeed flows in particular, skin friction drag can make up a large percentage of the overall drag on a vehicle. A specific example is in the combustor of a scramjet engine. In their review of the technological readiness of the design and use of hypersonic air-breathing engines for the National Aerospace Plane (NASP), Moszee and Snyder ${ }^{5}$ identified skin friction as an important, yet poorly understood, quantity. To illustrate the effects of skin friction on scramjet performance, Dr. F. S. Billig ${ }^{6}$ calculated the specific impulse of a generic Mach 10 hypersonic, scramjet-powered vehicle cruising at 94,000 $\mathrm{ft}(28,650 \mathrm{~m})$ with different values of $\mathrm{C}_{\mathrm{f}}$. Results are shown below in Table 1. Even small changes in $\mathrm{C}_{\mathrm{f}}$ cause significant changes in the specific impulse $\left(\mathrm{I}_{\mathrm{f}}\right)$. Heiser and $\operatorname{Pratt}^{7}$ indicate a similar result in their scramjet text. It is clear that accurate knowledge of skin friction is crucial in the design and testing of scramjet engines.

\begin{tabular}{|c|c|}
\hline $\mathbf{C}_{\mathbf{f}}$ & $\mathbf{I}_{\mathbf{f}}\left(\mathbf{l b}_{\mathbf{f}}{ }^{*} \mathbf{s} / \mathbf{l} \mathbf{b}_{\mathbf{m}}\right)$ \\
\hline 0.001 & 1670 \\
\hline 0.002 & 1540 \\
\hline 0.003 & 1410 \\
\hline 0.004 & 1280 \\
\hline 0.005 & 1160 \\
\hline 0.006 & 1030 \\
\hline 0.008 & 790 \\
\hline 0.010 & 560 \\
\hline
\end{tabular}

Table 1: Effect of Skin Friction on Specific Impulse of Scramjet Vehicle ${ }^{6}$

Although the working principle of the scramjet engine is simple, the flow-field in a scramjet is very complicated. The scramjet engine operates on the same principle as the ramjet, using the velocity of the aircraft to compress the air with no moving parts, unlike the rotating machinery found in a turbojet. The difference between the ramjet and the scramjet is that in the scramjet the flow remains supersonic throughout the engine, even in the combustor (hence, a Supersonic Combustion Ramjet $=>$ SCRamjet). In a typical scramjet, the flow is compressed in an inlet ramp and passes through an isolator before 
entering the combustor. Fuel is added in the combustor, and the hot gases are then expanded in a nozzle. With (fuel) injection, chemical reactions, shock-boundary layer interactions, and heat addition occurring, along with three-dimensional effects caused by the injection and the geometry of the injectors, the flow-field within the engine contains almost every complicating factor imaginable.

The tools available to predict skin friction in a scramjet engine become less and less reliable with increasing complexity. There have been some attempts at analytically extending existing correlations, such as those of Van Driest, Spalding and Chi, and White and Christoph, to high-enthalpy flows. Hazelton and Bowersox ${ }^{8}$ describe one method, applied to a scramjet inlet flow. They obtained results from one extension within $10 \%$ of the measured values of $\mathrm{C}_{\mathrm{f}}$, and only $20-25 \%$ in the other extensions. And, this was essentially for a $2 \mathrm{D}$, flat plate flow without any of the complications of the combustor (injection, mixing, separation, etc.). Computational methods and tools can be used with a fair amount of confidence to analyze incompressible and compressible laminar flows, and simple turbulent flows, but are increasingly being extended to analyze complex turbulent fluid flows that are hard to simulate. An accurate numerical prediction of such turbulent flows depends on good turbulence models. However, turbulence models developed to date still have much room for improvement, especially in the types of flows described above. Good experimental measurements of skin friction are therefore necessary to validate and/or refine future computational codes and their turbulence models ${ }^{9}, 10$.

\subsection{Specific Motivation: The Hyper-X}

Developing such tools is one of the primary goals of NASA's Hyper-X program (see Figure 1). This program is a scaled-back and more conservative carry-over from the cancelled NASP program. The program aims to validate the ground-based tools (windtunnel tests, CFD, heat and structural load simulations, etc.) for the design and analysis of hypersonic airframe-integrated scramjet vehicles by building a large database of information using those tools and then comparing them to actual flight data. The program will culminate in the flight-testing of three scramjet-powered vehicles, the first two at Mach 7, and the final flight at Mach $10^{11}$. The Hyper-X will be a key link between the decades of ground-based research into scramjet propulsion and the actual realization of scramjet powered flight ${ }^{12}$. 


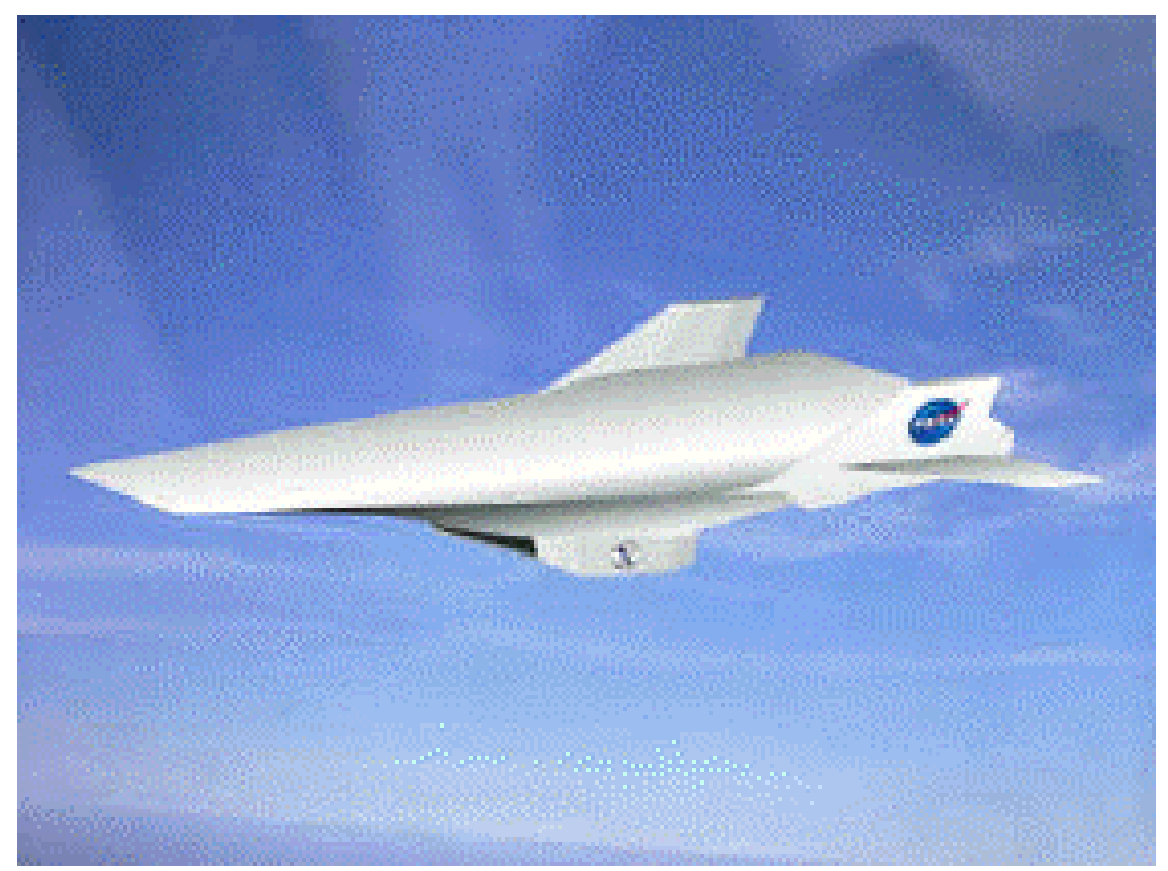

Figure 1: Artist's Sketch of Hyper-X Flight Vehicle ${ }^{13}$

The ground-based portion of the Hyper-X program has been going on since 1996. After an airframe-integrated scramjet vehicle design was selected, analysis was begun. A central portion of the ground-based testing consisted of wind tunnel tests of increasingly realistic models of the flight vehicle's scramjet engine. These tests are nearing completion for the Mach 7 configuration, with the first Mach 7 flight test attempted in June $2001^{11}$.

As stated above, skin friction is an important parameter in scramjet engines. It is therefore important to obtain measurements of skin friction both in the Hyper-X engine models tested on the ground, and in the actual flight vehicle, in order to compare with each other and with the tools used to predict it.

\subsection{Measurement Methods}

Skin friction has long been an item of practical concern to the scientist and engineer. Hence, Froude $^{14}$ performed the first well-documented experimental measurement of skin friction 130 years ago, by towing planks in a water tank at different speeds $^{15}$. The device he used is shown in Figure 2.

Since then, skin friction measurements have branched into two broad categoriesdirect measurements and indirect measurements. The local skin friction can be measured 
directly, by measuring the shear stress on the surface of the object, or indirectly, by measuring some other parameter such as pressure differences or heat flux and then relating those parameters to a value of skin friction through a correlating function. This section will briefly outline the differences, advantages and disadvantages of some of these methods.

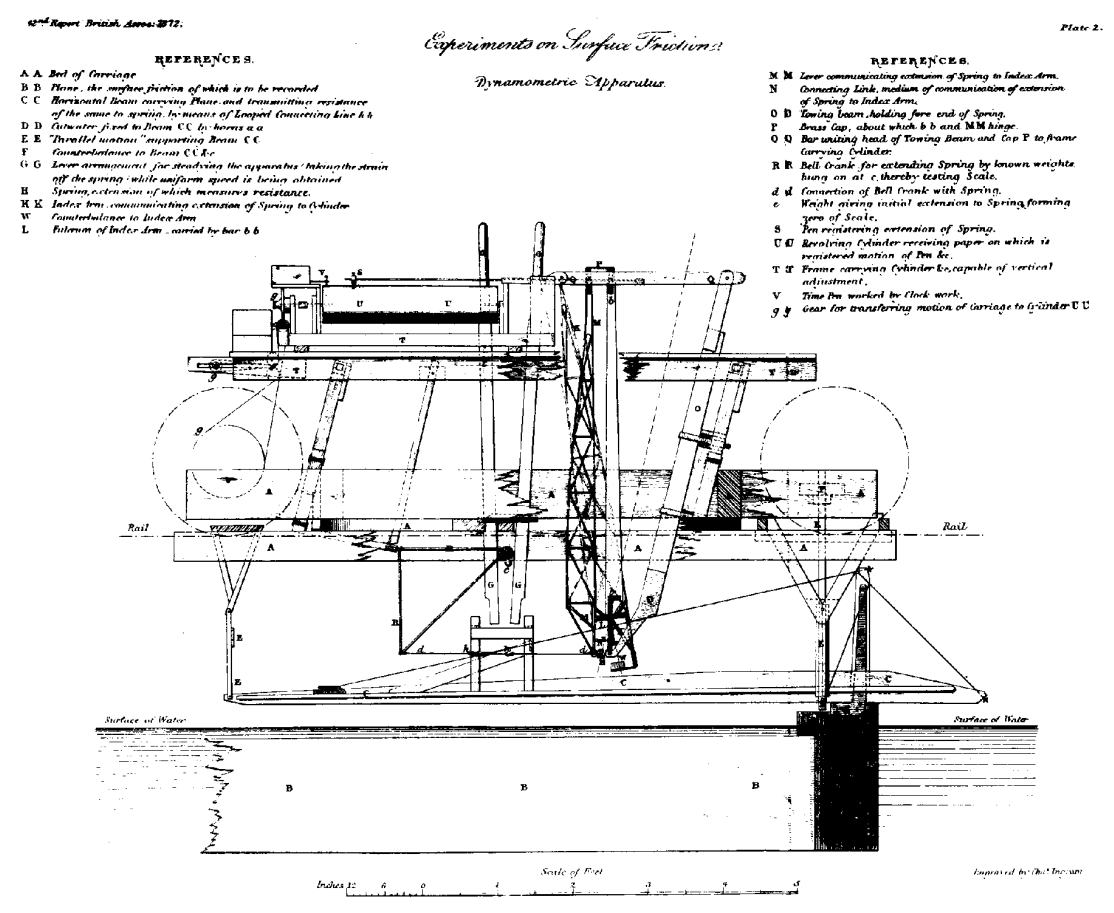

Figure 2: Measurement Device of Froude $\mathrm{e}^{14,15}$

\subsubsection{Indirect Methods}

Indirect methods of measuring skin friction use associating principles to relate skin friction to some other measured flow quantity. Nitsche, et al ${ }^{16}$ published a thorough review of several indirect methods. Figure 3, taken from that paper, graphically illustrates several of those methods and their respective correlating principles and calibrations.

The first of these in Figure 3a is representative of indirect methods that rely on analogy, in this case the Reynolds Analogy. The Reynolds Analogy relates skin friction to the heat transfer on the wall through the relation

$$
S t=\operatorname{Pr}^{-2 / 3} \frac{C_{f}}{2}
$$


where Pr is the Prandtl number, and St is the Stanton number. A surface hot-film is used in a constant-temperature anemometer circuit to relate the convective heat transfer to the shear stress through a calibration. The calibration of this instrument, as with many of the indirect methods, is dependent on flow conditions, particularly temperature ${ }^{16}$. A similar, but less popular, analogy relates the skin friction to mass transfer at the wall ${ }^{10,15}$.

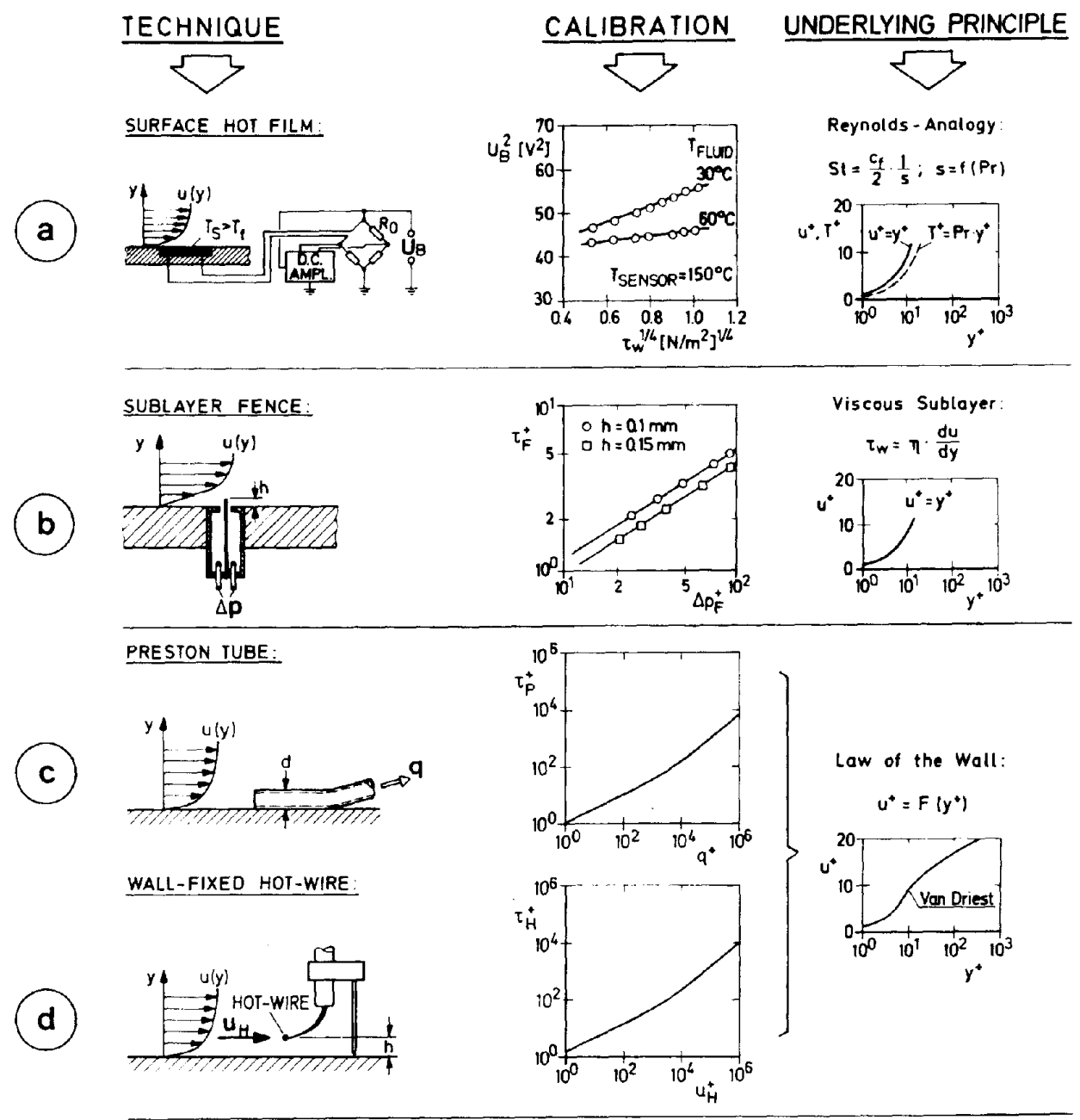

COMPUTATIONAL PRESTON TUBE:
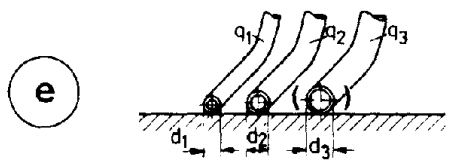

WALL-FIXED DOUBLE HOT-WIRE:
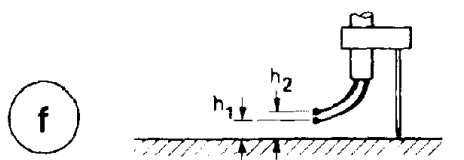

- NO CALIBRATION -

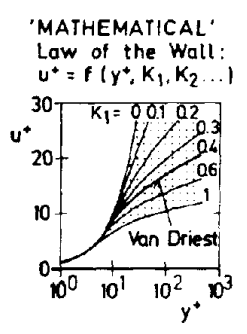

Figure 3: Indirect Skin Friction Measurement Techniques ${ }^{16}$ 
Flow around obstacles in the boundary layer is another major branch of indirect methods. These methods measure the pressure difference around an object in the boundary layer, and then relate it to the shear stress by using the similarity law of the viscous sub-layer:

$$
u^{+}=y^{+}, u^{+}=\frac{u}{u^{*}}, y^{+}=\frac{y u^{*}}{v}
$$

\section{Equation 4}

The pressure difference is then related to the shear stress by assuming a relationship between the pressure difference and the laminar sub-layer velocity profile. Figure $3 \mathrm{~b}$ shows one such technique, the sub-layer fence. Although the sub-layer fence can be used in flows with pressure gradients, and in compressible flows, its calibration requires foreknowledge of boundary layer parameters, and it is restricted to steady, twodimensional flows ${ }^{10}$.

The Stanton tube is another widely used device of this type. It has the advantage of being simple to construct, as it consists of a razor blade above a static pressure port. A schematic is shown in Figure 4. This creates a total pressure port that is able to be located within the laminar sub-layer, reducing compressibility and pressure gradient effects. The same caveats regarding the calibration of the sub-layer fence apply to the

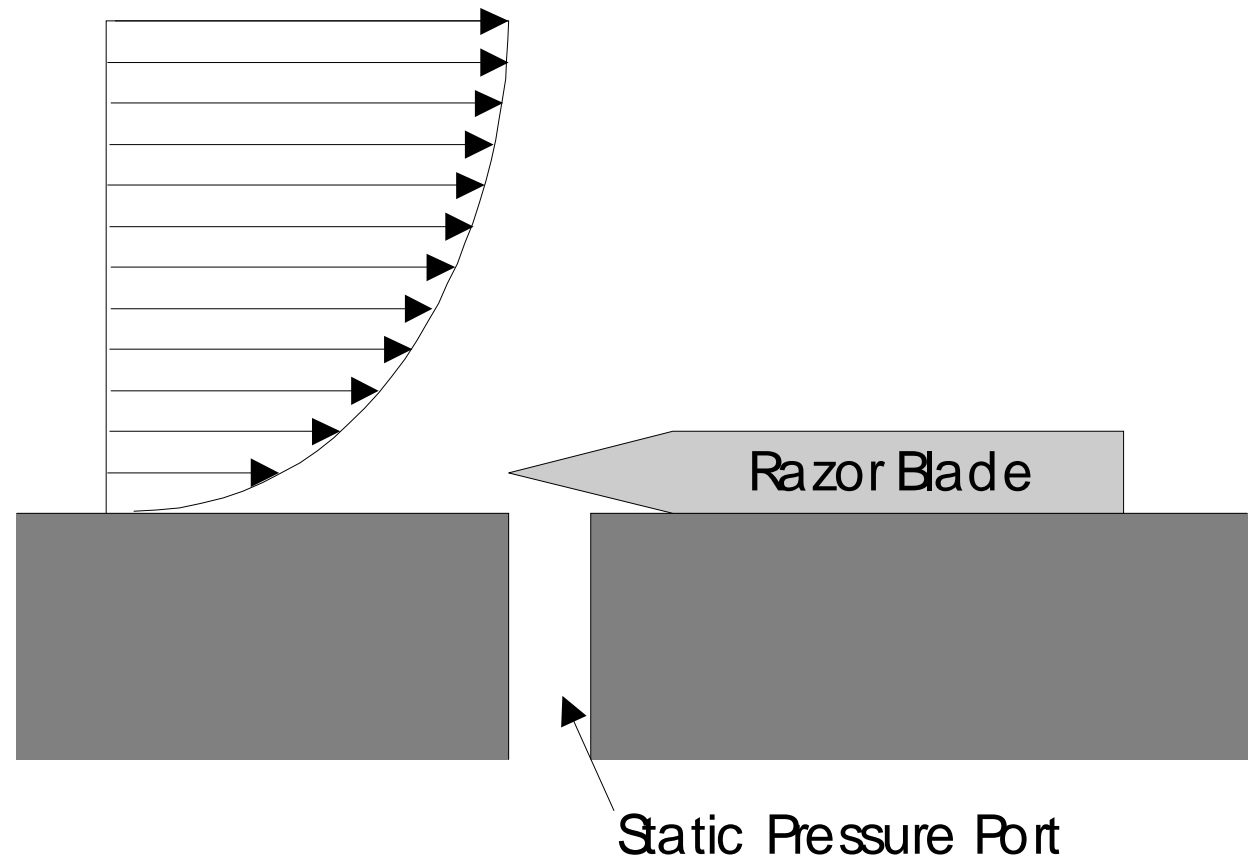

Figure 4: Stanton Tube ${ }^{10}$ 
Stanton tube, with additional concerns due to the size and positioning of the razor blade and the pressure orifice ${ }^{10}$.

Another class of indirect skin friction measuring methods consists of methods that measure the velocity profile in logarithmic region of the turbulent boundary layer. The velocity profile is then related to the skin friction through the law of the wall, which can be re-written as in Equation 5, such that $\mathrm{u} / \mathrm{U}$ can be written as a function of $\mathrm{yU} / \mathrm{v}$ with $u^{*} U=\left(C_{f} / 2\right)^{1 / 2}$ as a parameter. Plotting these lines produces a Clauser chart ${ }^{15}$.

$$
\frac{u}{u^{*}}=A \log \left(\frac{y u^{*}}{v}\right)+B=\frac{u}{U} \frac{U}{u^{*}}=A \log \left(\frac{y U}{v}\right)+A \log \left(\frac{u^{*}}{U}\right)+B
$$

\section{Equation 5}

While some techniques, like Laser Doppler Velocimetry, measure several velocities and use the Clauser chart, most of these techniques rather attempt to measure the velocity at a known distance from the wall ${ }^{10}$. The examples in Figure $3 \mathrm{c}-\mathrm{f}$ are of this type.

The Preston tube, which is probably the most widely used of all skin friction measurement techniques, is one such velocity profile method. The Preston tube consists of a circular Pitot tube resting on the wall. A calibration for the Preston tube is obtained that relates the pressure difference of the Pitot-static measurement, the diameter of the tube, and the fluid properties to the shear stress on the wall ${ }^{15}$. A Preston tube is sketched in Figure 3c.

All of these velocity profile methods are best suited for steady, two-dimensional, incompressible, turbulent flows, where the law of the wall is known to apply. In flows with transition, separation, compressibility, three-dimensionality, unsteadiness, or changing thermo-physical properties, or some combination of the above, these methods become less reliable. There have been attempts to extend these methods to some of these flows, most notably compressible and three-dimensional flows, with some success ${ }^{16}$. Yet the less that the law of the wall is known for these flows, the lower is the accuracy of these methods ${ }^{10,16}$.

A final type of indirect method is oil film interferometry. Although it may be considered to be a direct method of skin friction measurement, it is severely dependent on many flow factors, causing it to be categorized with indirect or "semi-direct" methods. 
With this method, a polished test article is coated with a thin film of oil, and laser interferometry is used to measure the thickness of the oil film. As the flow passes over the surface, the thickness of the oil film decreases, and the fringe pattern obtained from the interferometer is related to the thickness. The advantages of this method include good spatial resolution and a global measurement of skin friction, along with being nonintrusive to the flow. The disadvantages, however, are the sensitivity to temperature (due to the temperature dependence of the viscosity of the oil) and gradients in both temperature and pressure (due to the assumptions of the oil flow equations used). Also, it is necessary to align the oil film perpendicular to the streamlines of the flow, making it necessary to know flow direction beforehand. In addition, optical access to the test article is also necessary for the laser measurement. The oil film technique is useful in many simple cases, but cannot be used in complex, 3-dimensional, high-speed, and/or high-enthalpy flows. ${ }^{10}$

\subsubsection{Direct Methods}

Direct methods of measuring skin friction measure the tangential force due to the shearing of the flow by separating a small element, referred to as a floating element, from the wall. These techniques have several advantages over indirect methods. First, the device will measure the shear regardless of the flow parameters. Therefore no prior knowledge of the flow is necessary in order to substantiate a correlation or calibration for the technique. In principle, laminar, transitional, turbulent, or separating flows, with or without compressibility and/or chemical reactions could all be measured with the same device. Even flows with injection, suction, or shock-boundary layer interactions have been tested with this method. Direct measuring skin friction gages have also been successfully extended to three-dimensional flows ${ }^{3}$. In addition, a direct measurement, since it is flush with the wall, is non-intrusive to the flow.

The first known direct measuring skin friction sensor was that of Froude, shown earlier. Kempf (1929) and Schoenherr (1932) were the next most notable researchers to use the direct measuring method. Their work became the basis for the now common estimates for incompressible skin friction. However, the application of direct measurement techniques to high-speed flows really came into focus in 1953 with the

seminal work of Dhawan ${ }^{17}$ and Coles ${ }^{18}$. The floating element design of Dhawan has been 

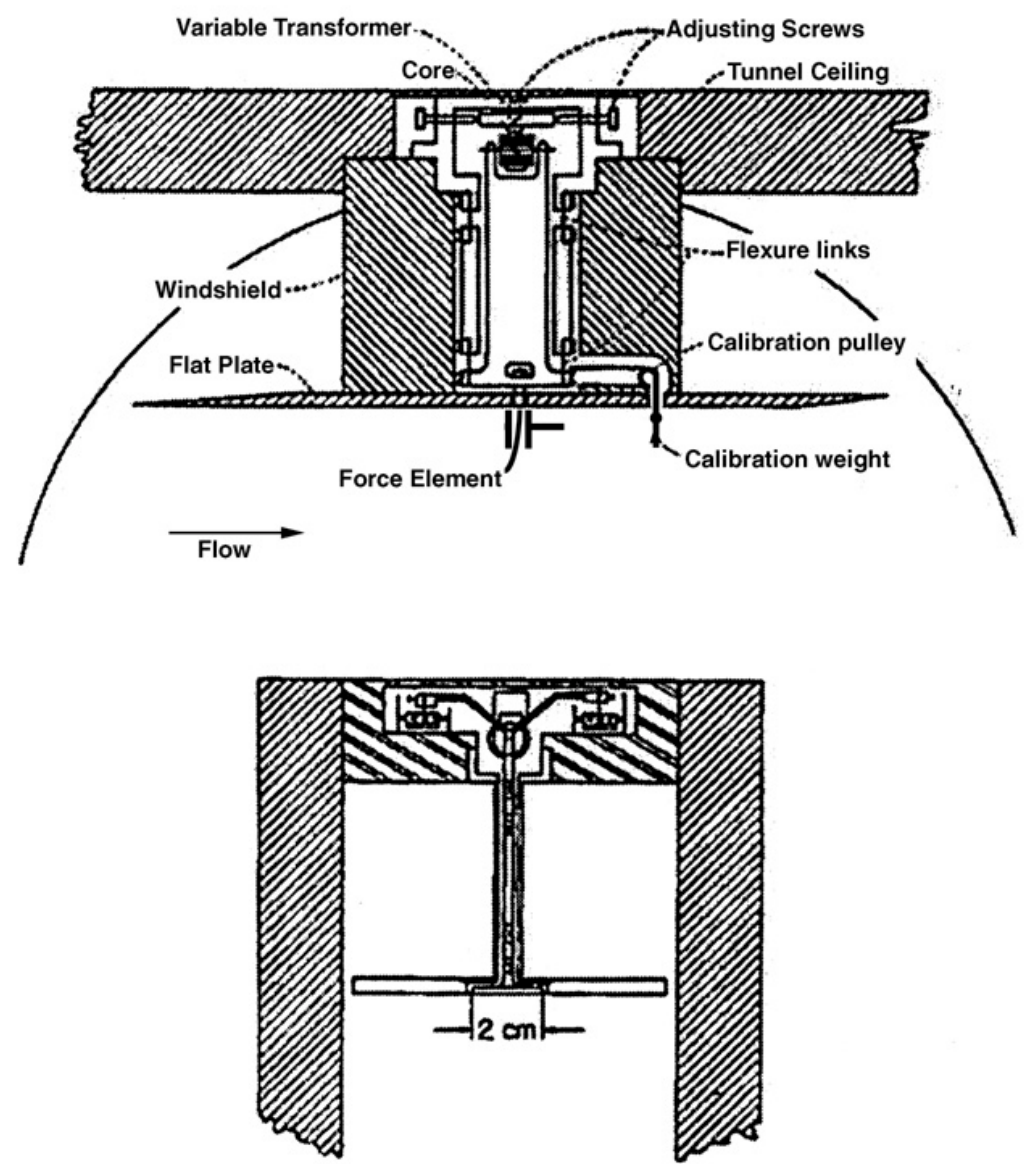

Figure 5: Dhawan's Skin Friction Balance

the basis of many direct skin friction balances, having identified most of the important design parameters. A schematic of his device is shown above in Figure 5. The work that followed, including that of Allen ${ }^{19,20}$, Voisinet ${ }^{21}$, and Roensch and Cadwell ${ }^{22}$, often used similarly complicated linkage mechanisms to support the floating element.

Many of these designs were of the nulling type, meaning that as the wall shear deflected the floating head, a restoring force was used to return the floating head to its null position in order to prevent errors due to misalignment. The restoring force was then monitored since it should be equal to the shear force acting on the floating element. These nulling types of instruments, while they reduce some uncertainty from the measurements, had the disadvantages of being complex, unreliable, and slow to respond to flow phenomena. Figure 6 shows an example of a nulling design.

Non-nulling skin friction gages allow the floating head to be deflected, and do not provide for a restoring force. This leads to smaller, simpler and more robust designs, and 


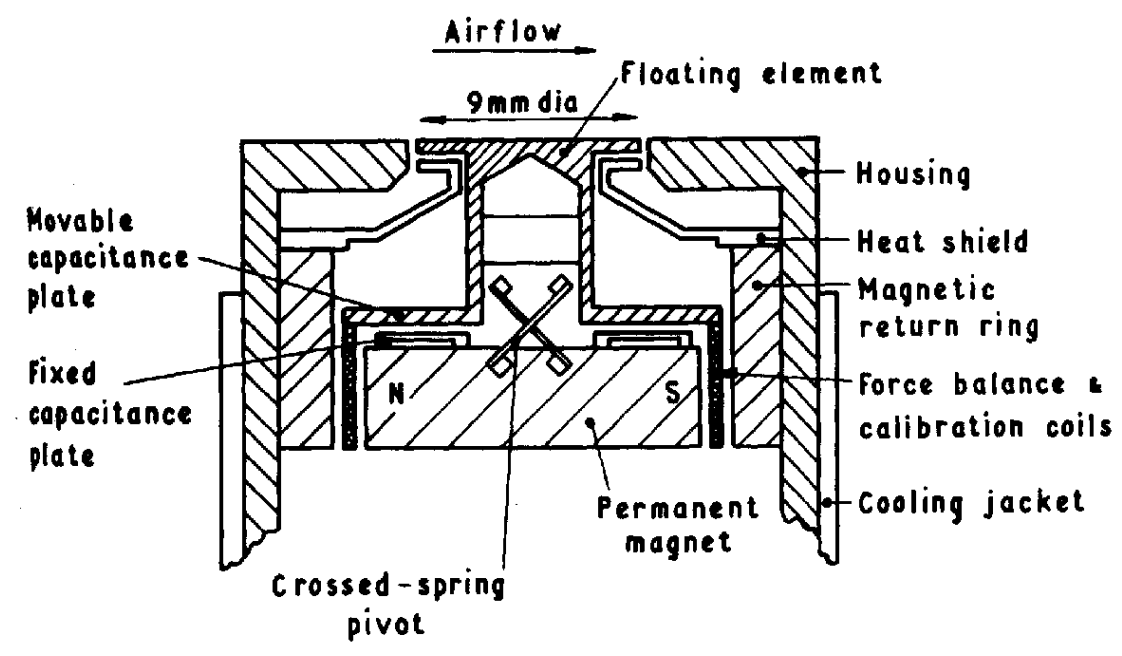

Figure 6: Example of Nulling Skin Friction Gage of Kistler (from Paros ${ }^{28}$ )

improves frequency response, at the cost of possible misalignment errors. Early designs of both the nulling and non-nulling type often used Linear Variable Differential Transformers (LVDTs) to measure the displacement of the floating element; in the nulling designs the signal was used to the determine the correct restoring force, while in non-nulling types the signal was used directly as the output. Skin friction sensors were made even more simple and small when strain gages, especially the semi-conductor type, began to be used as the displacement sensors, since they have greater sensitivity and a smaller size than LVDTs. By then being able to measure small deflections, and hence keeping the displacement of the floating element very small, the non-nulling method could be used without incurring much misalignment error. One of the first of these types was that of Schetz and Nerney ${ }^{23}$. Since then, they have been used in many different facilities, including high-enthalpy scramjet wind tunnel tests ${ }^{24,25}$, extremely shortduration impulse wind tunnel tests ${ }^{26,27}$, and transonic flight tests ${ }^{1}$.

Thorough histories of direct skin friction measurements are given by Winter ${ }^{15}$ (through the mid-1970s) and Magill ${ }^{29}$ (through 1999). Pulliam ${ }^{10}$ summarizes the general methods available for measuring skin friction discussed above, both direct and indirect, and lists the relative advantages and disadvantages of each.

\subsection{Scope of the Investigation}

Measurements of skin friction have been made in scramjet combustors in ground tests, as mentioned previously. Also, skin friction gages have been implemented on high- 
speed flight vehicles in the past. Among those is the instrument of Lyons ${ }^{30}$ (1957), which was made to be insensitive to the linear and angular accelerations that are inherent in a flight test. His gage was developed for testing on an Aerobee-Hi rocket, and is shown below in Figure 7. Skin friction measurements were also part of NASA's YF-12 experiments in the 1970s. Reports by Fisher ${ }^{31}$, as well as Quinn and Gong ${ }^{32}$, illustrate measurements made at Mach 3 on the surface of the YF-12 aircraft, and in a hollow cylinder suspended from the aircraft, respectively. Fisher compared the results of a direct-measuring balance with those of a Preston tube measuring in a nearby location. The more recent work of Sang ${ }^{1}$ produced a gage that was tested on a fixture suspended beneath an F-15 at transonic speeds.

The work that is described in the following chapters was aimed at developing direct measuring, non-nulling skin friction sensors that could be tested in the scramjet engine of the Hyper-X flight vehicle (X-43A). As discussed earlier, methods of predicting skin friction need to be verified by experimental measurements in scramjet engines, and indirect methods become unreliable in very complex flows. This implies the

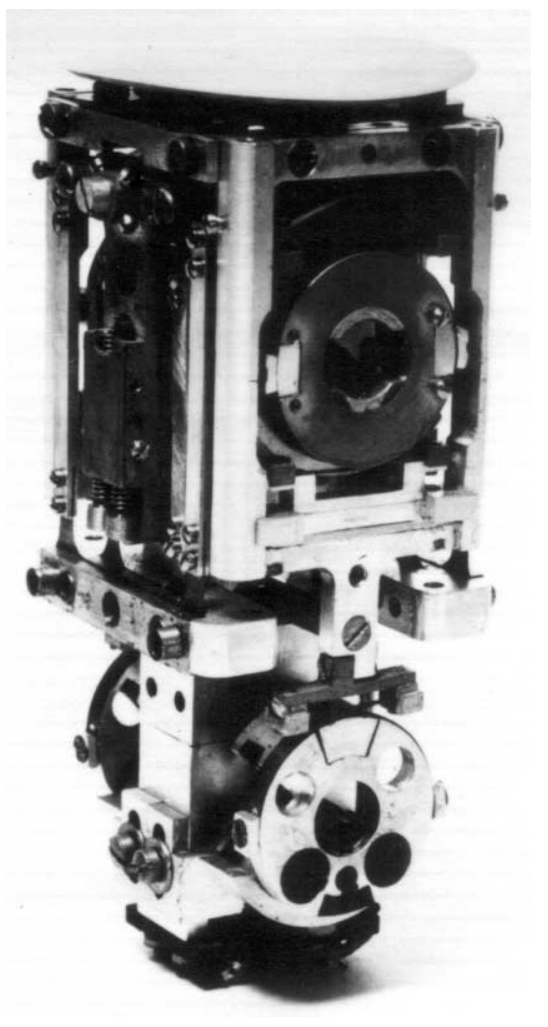

Figure 7: Acceleration Insensitive Skin Friction Balance for Flight Tests (Lyons) ${ }^{30}$ 
need for a direct-measuring skin friction gage. A non-nulling design approach was selected because of the better time response and greatly reduced complexity and size compared to a nulling sensor. And, with the ability to measure very small deflections with strain gages, it was possible to design sensors with negligible floating head displacement effects.

As the Hyper-X is the first scramjet integrated flight vehicle ever to be flown, these sensors will be the first skin friction sensors to have been tested on a scramjet flight vehicle. They will combine the efforts of previous free-flight skin friction balances, and skin friction gages tested in scramjet combustor ground tests.

The development of the gages for this work fell into three major steps. The first step was to design and test a skin friction gage that could be tested in a scramjet engine ground test. This led to tests in the GASL Leg IV facility, where measurements were made in rocket-based-combined-cycle (RBCC) engine operating in scramjet mode. The second step was to improve on the first design, and implement it in the Hyper-X Engine Model (HXEM). This model is a full-scale, partial-width mock-up of the Hyper-X scramjet engine. These tests were conducted in the NASA Langley Arc-HeatedScramjet-Test-Facility (AHSTF). The last step was developing the gage for the actual flight vehicle. It was originally planned that this third gage would be an evolution of the first two. However, more stringent requirements for flight vehicle hardware, as well as some problems discovered with the second design, led to a very different design for the flight vehicle gage. It too, therefore, was tested in the NASA Langley AHSTF in the Hyper-X Engine Model (HXEM) in order to verify its operability in a scramjet engine environment, before installing it in the flight vehicle engine.

Various other tests were done on each of these gages, including calibration, vibration shaking, and cold-flow supersonic wind tunnel tests. The flight vehicle gage was also subjected to heat flux and environmental pressure and temperature tests. These tests, as well as the design and development of each of the three gages will be discussed in the following chapters. 


\section{CHAPTER TWO:}

\section{DESIGN OVERVIEW AND CONSTRAINTS}

\subsection{General Design Attributes}

The general design of the sensors for this study was that of a non-nulling floating element, direct measuring skin friction gage. A simple sketch shown in Figure 8 illustrates the working principle and identifies the major components. The flow moving across the surface of the floating head exerts a force on it. This is caused by the shearing stress being integrated over the area of the floating head. By attaching the floating head to a flexible member with strain gages arranged in a Wheatstone bridge, the deflection of the floating element can be sensed, producing an output that is proportional to the wall shear, $\tau_{\mathrm{w}}$. The skin friction coefficient is then calculated by dividing the wall shear stress by the local dynamic pressure, $\mathrm{q}$, as shown in Equation 2. As will be seen later, different types of flexures were used in the different designs.

Since the shearing stresses in these flows are relatively small, typically on the order of 100 to $500 \mathrm{~Pa}$ (2.09 to $10.4 \mathrm{psf}$ ), it is important to develop a floating head and flexible member that are both sensitive and yet compact and non-intrusive. Having a larger floating head surface area increases the amount of force transmitted to the flexible member, but has the obvious disadvantage of increased size and mass. To allow a

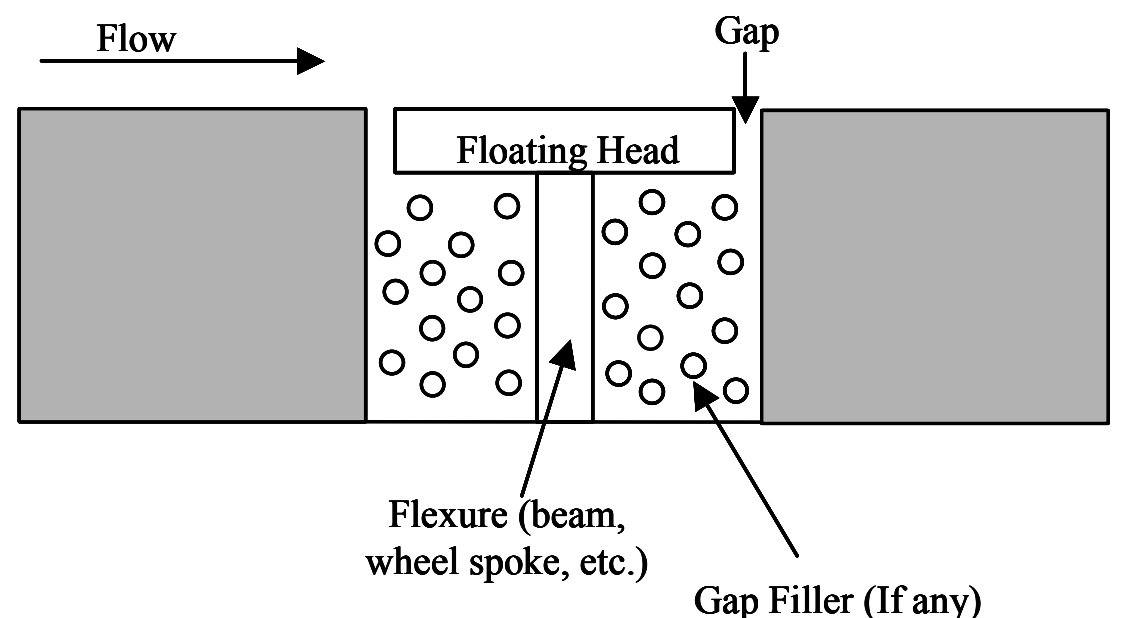

Figure 8: Skin Friction Gage General Features 
smaller floating head to be used, a more flexible member might be proposed. However, if a cantilevered beam is used as the flexible member, this often means a longer beam, which increases the size and lowers the natural frequency of the instrument. Vibration damping of such structures then becomes an additional concern. Also, the flexible member must remain stiff enough to keep the displacement of the floating head small with respect to the size of the gap (usually on the order of 0.005 inches, or $0.127 \mathrm{~mm}$ ). These are just some of the basic design issues that must be addressed, and they illustrate the trade-offs that must be made. The gages that were built for this study were specifically designed for operation in scramjet engine models. This environment dictated many of the specific design features and constraints.

The engine models in which the gages were to be tested were of the heat sink variety. This meant that they were not actively cooled, but instead were made of copper and intended to simply absorb the heat flux induced by the high-enthalpy flow. It was important to match the properties of the floating element to those of the surrounding engine wall as closely as possible, so as to prevent errors due to temperature mismatch between the floating element and the surrounding engine wall. Voisinet's ${ }^{21}$ study showed that such a temperature mismatch could lead to significant errors in the measurement of skin friction. By providing a heat flow path as much like the rest of the engine wall as possible, this error can be minimized. For this reason, it was determined that the floating element of the skin friction gage should be made from copper and be one inch in length, since the engine wall was also one inch thick.

The high levels of heat transfer to the engine walls also meant that the design would need to provide for thermal protection for the electronics of the sensor, and that care would have to be taken to minimize erroneous readings due to temperature sensitivity of the strain gages. This was addressed differently in the different designs, as will be seen in the subsequent chapters.

Since the incorporation of skin friction gages was not a part of the original design of some of the engine models in which the same gages were ultimately tested, it was necessary to design the first skin friction gage to mount into existing instrumentation holes in the engine model. After the success of the first skin friction gage, and due to the fact that all the engine models were built by the same manufacturer, all of the subsequent 
gages were designed around the same mounting configuration. This mounting configuration was taken, as stated above, from an existing instrumentation access port, in this case for a Gardon-type heat flux gage, built into one of the engine models.

Additional motivation for using these same ports was the advantage of allowing comparisons between measurements of heat flux and skin friction at the same location in the scramjet engine. These comparisons could then be used to better understand the correlation between heat flux and skin friction in this more complicated flow environment. Unfortunately, the Gardon heat flux gages did not perform well, and the originally planned comparisons were not made. However, the instruments developed during this work for the Hyper-X Research Vehicle included not only a skin friction gage, but also a heat flux gage. These used the same access holes as the previous gages, and hence were able to be tested in the HXEM.

Figure 9 shows the arrangement of the mounting hole used for the skin friction gages in this study. It included a primary access hole of 0.368 inch $(0.935 \mathrm{~cm})$ diameter and one inch $(2.54 \mathrm{~cm})$ length, with fastening holes drilled and tapped for $1 / 4-20$ screws located on either side at 0.455 inches $(1.156 \mathrm{~cm})$. An O-ring groove was incorporated at the base of the primary access hole. The holes were located within an oval-shaped cutout 0.250 inches $(0.635 \mathrm{~cm})$ deep, with width of 0.750 inches $(1.905 \mathrm{~cm})$ and ends of radius 0.375 inches $(0.9525 \mathrm{~cm})$.
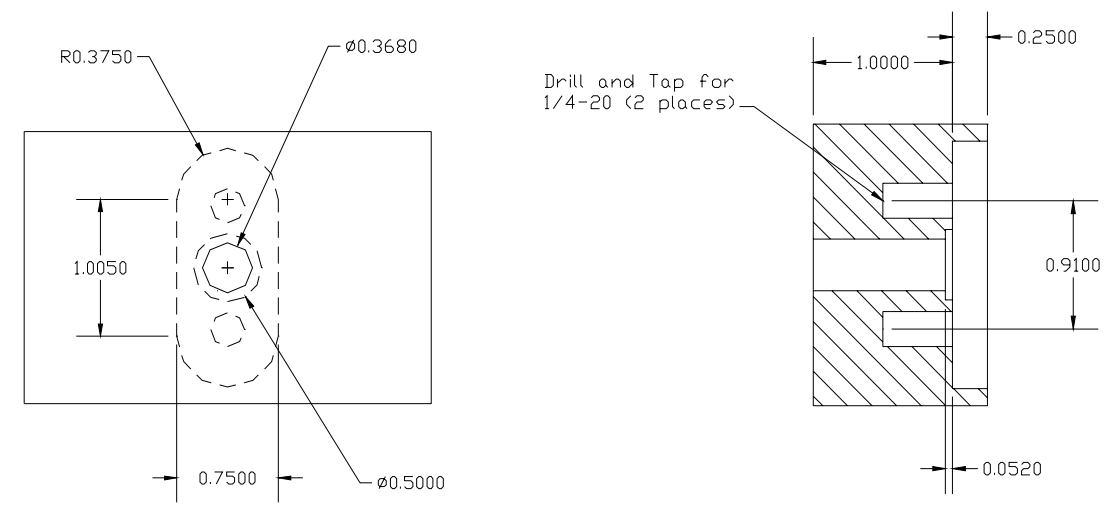

Figure 9: Mounting Hole for Hyper-X Skin Friction Gages 


\subsection{Floating Head Design Attributes and Concerns}

The shape of the primary access hole was the driving factor in the design of the floating head in all three designs in this study. The head was cylindrical in shape, made of copper, and one inch $(2.54 \mathrm{~cm})$ in length. For the first two designs, a housing was used to support and protect the floating element and flexible member. This housing fit into the main access hole, and the floating element was then located within this housing. The diameter of the floating element at the surface was 0.300 inches $(0.762 \mathrm{~cm})$ in these designs. The third design did not extend the housing around the floating head, allowing a larger diameter of 0.348 inches $(0.884 \mathrm{~cm})$.

A typical floating head configuration is shown in Figure 10. It has long been known that the floating head geometry is important to a successful direct measuring skin friction gage, leading to several studies of the effects of floating head geometry on measurement uncertainties ${ }^{33,34}$. Some of the more notable work was done by Allen ${ }^{19,20}$ in the 1970's. He identified important floating head geometric parameters and studied their effects, including the effects of misalignment of the floating head, on the error in direct skin friction measurements. These geometric parameters are shown in Figure 11, where $\mathrm{G}$ is the gap size, $\mathrm{C}$ is the lip size, and $\mathrm{Z}$ is the misalignment. Positive $\mathrm{Z}$ indicates protrusion of the floating head above the surrounding wall, while negative $Z$ indicates recession of the floating head below the surface of the surrounding wall. The diameter of the floating head is D.

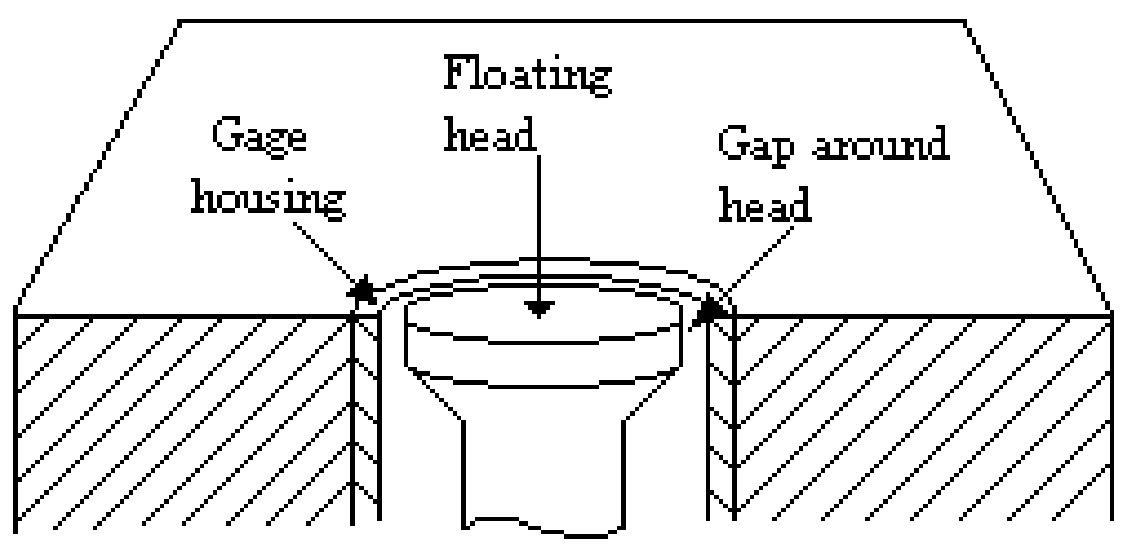

Figure 10: Typical Floating Head Configuration 


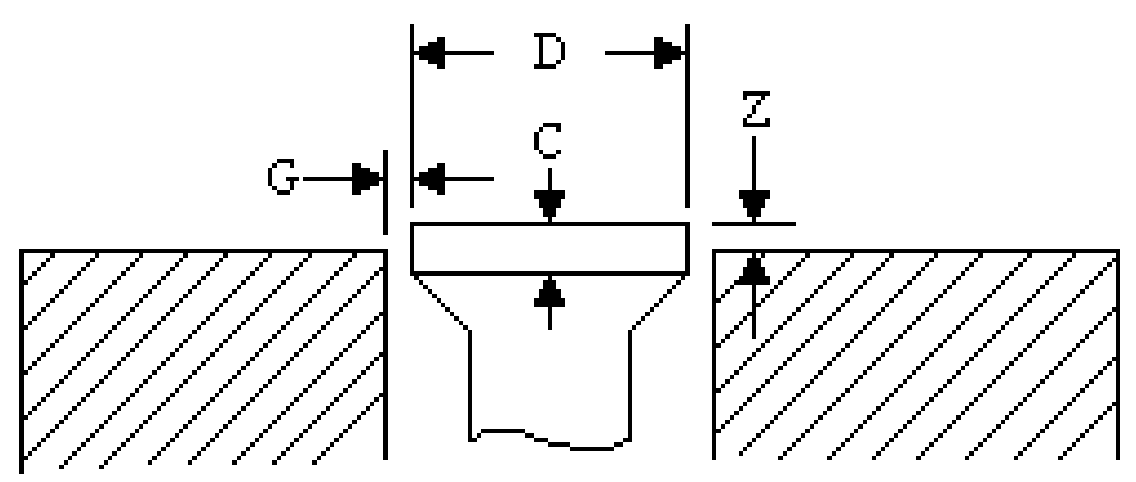

Figure 11: Floating Head Geometric Parameters

The geometry of the floating element is important, because there are multiple forces from the flow that may act on it. These forces, as shown in Figure 12, are the lip force, the normal force, and the shear force. It is desired that the floating head only be sensitive to the shear force. Lip forces or off-center normal forces acting on the floating head will cause an error in the skin friction measurement due to an increased or decreased amount of force, or an induced moment about the flexible member, respectively. These forces are increased when there is misalignment, as the altered flow path creates pressure differences across the head and between its upper and lower surfaces. Pressure gradients in the flowfield can also cause pressure differences acting on the lip and/or an off-center normal force, which can lead to a moment about the pivot point of the flexible member.

Allen found that while a perfectly aligned floating head would have minimal error, using optimal ratios of the different geometric parameters could reduce the errors caused by misalignment ${ }^{20}$. Specifically, his results indicated that misalignment errors

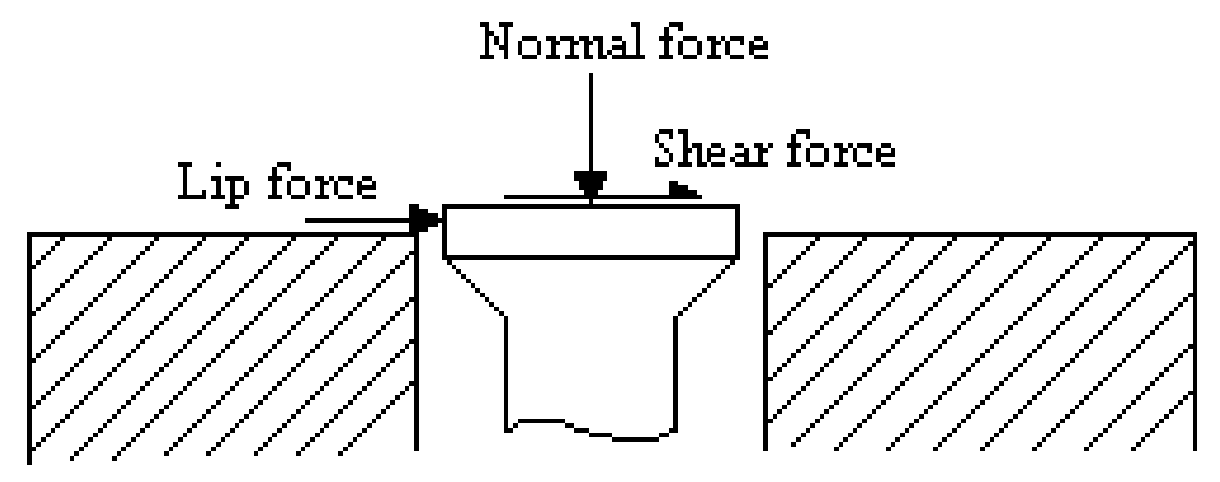

Figure 12: Forces on Floating Head 
were reduced with increasing gap-to-diameter (G/D) ratio. For the first two designs built for the current study, the G/D ratio was 0.0167 , and the third design had a G/D of 0.0144 . These values were even larger than the ratios tested by Allen (0.001 to 0.010$)$. Reducing the lip-to-diameter (C/D) ratio was also shown to decrease the effect of misalignment errors. The $\mathrm{C} / \mathrm{D}$ ratios for the gages in the current study were relatively large, 0.10 for the first two designs, and 0.0862 for the third design. However, the reduction of errors with increasing $\mathrm{G} / \mathrm{D}$ ratio noted above was obtained with the largest $\mathrm{C} / \mathrm{D}$ ratio. Also, the misalignment in Allen's work was normalized by the boundary layer thickness, $\delta$. Given the estimated thickness of the boundary layer, and the estimated amount of misalignment for these sensors, as well as the large $\mathrm{G} / \mathrm{D}$, the errors due to misalignment should be very small for these designs (see Appendix A for Uncertainty Analysis and more discussion).

It is also important to note that there are differences between the experiments of Allen and the types of testing undergone by the sensors in this study. Allen's work was done in cold supersonic flow, with a relatively large, nulling skin friction balance. The smaller, non-nulling design operating in high enthalpy flows may have different error sensitivities. Also, various researchers have addressed some of the concern about misalignment and pressure gradient effects by filling the gap with an incompressible fluid, such as silicon oil. This eliminates the possibility of pressure gradients between the upper and lower surface of the floating head, and, if the oil remains at the same level as the floating head, prevents pressure differences from acting on the lip. Allen did not use a gap filling fluid, as was done in the first two designs in this study, so Allen's work is here primarily used as a guide, not necessarily as a strict design rule.

Since a non-nulling design was used for this study, an additional concern related to the floating head was the displacement undergone by the floating head. In the first two designs of this study, a cantilever beam was used as the flexible member. Under the action of a load, the floating head, positioned at the free end of the beam, undergoes not only a lateral deflection, but also an angular tilting. This tilting could potentially cause the head to protrude on one side, and be recessed on the other side. The solution of this problem was simply to design the beam to be stiff enough to make the displacement and tilting negligible. This was done by using conventional beam theory to analyze the beam design under expected loads, as illustrated below. 
The sensor was modeled as a cantilevered beam with a load, P, acting at the free end, where the load $\mathrm{P}$ is equal to the wall shear stress multiplied by the surface area of the floating head. The length of the beam is $L$, and a reaction moment, $M_{R}$, is generated at the base equal in magnitude to the product of $\mathrm{P}$ and $\mathrm{L}$,

$$
M_{R}=P \times L
$$

\section{Equation 6}

$$
M(x)=M_{R} \times x
$$

\section{Equation 7}

An equation can then be written for the moment in the beam as a function of distance from the base, as shown in Equation 7. The moment distribution is then used to find the deflection by integrating Equation 8 twice, where $\mathrm{Y}$ is the transverse deflection of the beam, $\mathrm{E}$ is the modulus of elasticity of the material, and $\mathrm{I}$ is the area moment of inertia of the beam cross-section. This was carried out sequentially for the different segments of the beam, each of which had a different area moment of inertia and/or material, using the appropriate boundary conditions at the ends of beam (no slope at the base, and zero moment at the free end), and at the interfaces between the different segments (continuous deflection and slope).

$$
\frac{d^{2} Y}{d x^{2}}=\frac{M(x)}{E I}
$$

\section{Equation 8}

Performing the above analysis on the cantilevered beam used in the first two designs of this study yielded a predicted deflection of the floating head of about 0.0001 inch $(0.00025 \mathrm{~cm})$ and a protrusion of about 0.00001 inch $(0.000025 \mathrm{~cm})$. This deflection and protrusion was considered negligible compared to the gap size of 0.005 inch $(0.0127 \mathrm{~cm})$.

\subsection{Strain Gage Theory and Practice}

\subsubsection{Strain Gage Fundamentals}

As was stated earlier, strain gages mounted on the flexible member were used to sense the deflection of the floating head. The strain gages actually measure the strain, or the change in length of the test specimen normalized by the unstressed length. When a 
linear structure, such as a cantilever beam, is used, the strain is directly proportional to the force acting on the structure. The strain is given by

$$
\varepsilon=\frac{\sigma}{E}
$$

\section{Equation 9}

where $\varepsilon$ is the strain, $\sigma$ is the stress, and $\mathrm{E}$ is the modulus of elasticity of the material. The stress at the base of the beam is found from Equation 10, where $\mathrm{P}$ is the applied load, $\mathrm{L}$ is the beam length, $\mathrm{c}$ is the thickness of the beam, and I is the area moment of inertia of the beam cross-section. By substituting $\sigma$ from Equation 10 into Equation 9, strain is seen to have a linear relationship to the force on the end of the beam. It is also clear that by knowing an expected force, it is possible to optimize the beam geometry such that a measurable strain is obtained, while at the same time keeping the deflection of the end to a reasonable minimum.

$$
\sigma=\frac{P L^{c} / 2}{I}
$$

\section{Equation 10}

Electrical resistance strain gages were used in the skin friction sensors of the current work. These strain gages are very common in countless force measurement applications, and they are commercially available in a wide variety of shapes, sizes, and environmental capabilities. The operating principle is simple; the strain gage typically consists of a conductor pattern on a thin backing material, which is firmly bonded to the test specimen. When the test specimen is strained, the strain is directly communicated to the strain gage. The wire of the strain gage is then compressed or lengthened, and the resistance of the strain gage either decreases or increases due to dimensional changes of the conductor and the fundamental material property called piezoresistance. Piezoresistance is the dependence of a material's resistivity on mechanical strain ${ }^{35,36}$.

Strain gages are usually arranged into a Wheatstone bridge to increase the amount of output available from the circuit, and to provide compensation for other effects such as temperature sensitivity. A typical Wheatstone bridge circuit is shown in Figure 13. An excitation voltage, $\mathrm{E}_{\mathrm{i}}$ is required to power the circuit, and an output voltage, $\mathrm{E}_{0}$, is measured. The four resistors that make up the arms of the circuit are labeled $R_{1}, R_{2}, R_{3}$, 


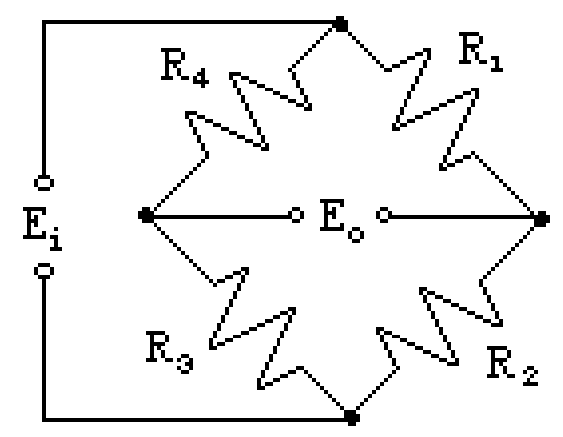

Figure 13: Wheatstone Bridge

and $\mathrm{R}_{4}$. It is possible for one, two, or all four of these resistors to be active strain gages (active meaning that the resistance will change with loading) while the other resistors simply provide completion of the bridge and maintain constant resistance. These different bridge arrangements are called "quarter-," "half-," or "full-," Wheatstone bridges, respectively. The skin friction sensors of this study used either half or full Wheatstone bridges.

$$
\frac{E_{o}}{E_{i}}=\frac{R_{1}}{R_{1}+R_{2}}-\frac{R_{4}}{R_{3}+R_{4}}
$$

\section{Equation 11}

The output from a Wheatstone bridge can be written as shown in Equation 11. If the changes of resistance in adjacent arms, such as $R_{1}$ and $R_{4}$, are of opposite sign, the effect on bridge output is additive, but if the resistance changes are of the same sign, the net effect on bridge output is subtractive. On the other hand, resistance changes in opposite arms of the bridge, such as $\mathrm{R}_{1}$ and $\mathrm{R}_{3}$, have the opposite characteristic. It is also clear from Equation 11 that if

$$
\frac{R_{1}}{R_{1}+R_{2}}=\frac{R_{4}}{R_{3}+R_{4}}
$$

Equation 12

then the bridge is balanced, and the output is zero. Any change in the resistance of one or more of the strain gages will cause unbalancing of the bridge, and a resultant output.

$$
G F=\frac{\Delta R}{R \varepsilon}=1+2 v+\pi_{1} E
$$

\section{Equation 13}


Equation 13 shows the definition of the gage factor, GF, which is the ratio of change in resistance of the strain gage to the strain, $\varepsilon$, and it is dependent on the material properties of the strain gage ${ }^{37}$.

In Equation 13, the first term on the right hand side represents the resistance change due to the length change of the strain gage; the second term represents the resistance change due to the area change (where $v$ is the Poisson's ratio of the material); and the last term represents the resistance change due to the piezoresistance effect (where $\pi_{1}$ is the longitudinal piezoresistance coefficient, and $\mathrm{E}$ is the modulus of elasticity). Gage factor is an indication of the sensitivity of the strain gage; typical values generally range from about 2, for common metal foil strain gages, up to about 150 for semiconductor strain gages ${ }^{36,37}$.

When two active strain gages are placed one each on opposite sides of a beam structure and in adjacent arms of the Wheatstone bridge, a "bending half bridge" is formed. In this configuration, as the beam bends, opposite resistance changes are induced in the strain gages, as one is in tension and the other is in compression. Their individual contributions are added, effectively doubling the output of a single strain gage. An axial load on the beam will cause either tension or compression in both strain gages, causing the same resistance change in each, which then cancels in the bridge circuit. The output for a bending strain in this configuration can be written as

$$
\frac{E_{o}}{E_{i}}=\frac{\varepsilon \times G F}{2}
$$

\section{Equation 14}

where $\varepsilon$ is the strain in the beam at the location of the strain gages. A similar arrangement places two strain gages next to each other on each side of a beam for a total of four strain gages, with the pairs on the same side in opposing arms of the Wheatstone bridge. This is the "bending full bridge." By incorporating all four arms of the Wheatstone bridge, the output, as shown in Equation 15, is double that of the half bridge configuration ${ }^{35,37}$.

$$
\frac{E_{o}}{E_{i}}=\varepsilon \times G F
$$

\section{Equation 15}




\subsubsection{Strain Gage Types and Selection}

The first two skin friction sensor designs developed in the current study employed semi-conductor strain gages applied at the base of a cantilever beam. The main reason for this was the fact that semi-conductor strain gages have much higher gage factors than standard metal foil strain gages. This allowed the beams to be designed to be stiff enough to keep the deflections small, but still undergo enough strain for the strain gages to produce a sensible output.

Gage factors for semi-conductor strain gages are typically about 150 . Looking at Equation 13, it is clear that since $v$ is between 0 and 0.5 for all materials, the piezoresistance of semi-conductor strain gages is the dominant contributor to gage factor, causing them to often be called piezoresistive strain gages. Since the material property $\pi_{1}$, the longitudinal piezoresistance coefficient, can be either positive or negative, semiconductor strain gages can be made with either positive or negative gage factor. Unfortunately, semi-conductor strain gages are more sensitive to temperature, and have increased non-linearity compared to standard metal foil strain gages ${ }^{36}$.

The semi-conductor strain gages selected for the first two designs were obtained from Micron Instruments, model number SS-060-033-500P-S(4). These strain gages had nominal gage factor of 140 , and resistance of $500 \Omega$. The size of the strain gages was very small, only 0.060 inch $(0.152 \mathrm{~cm})$ long including the backing material. Because of the small size, and also due to installation difficulties associated with semi-conductor strain gages, the skin friction sensor beams were shipped to the strain gage manufacturer for custom installation and wiring.

The final skin friction sensor design developed in this study (X-43A flight vehicle design) employed metal foil strain gages. These strain gages are the most widely used of the strain gage varieties, with typical gage factors of about 2. From Equation 13, it is clear that the sensitivity of these gages is due almost entirely to the geometric changes caused by strain. While they are also subject to thermal errors, they are much less so than the semi-conductor types. Since they are more widely used, a larger base of knowledge and experience exists. Also, the metal foil strain gages selected for this study were larger than the semi-conductor strain gages. These factors made them far easier to work with, allowing for on-site installation and wiring of the strain gages. 
The metal foil strain gages used on the final skin friction sensor design were manufactured by Micro-Measurements. These gages were made of constantan with a gage factor of 2.095, and nominal resistance of $350 \Omega$. They were selected for small size, having a total length of 0.125 inch $(0.3175 \mathrm{~cm})$, and a thermal expansion coefficient matched to that of the aluminum to which they were to be mounted.

\subsubsection{Strain Gage Thermal Effects}

Electrical resistance strain gages are susceptible to errors due to temperature. There are multiple reasons for this fact. One is that the resistance of the strain gage is sensitive not only to changes in strain, but also in changes in temperature, with the resistance changes due to temperature sometimes overshadowing the changes due to strain. The sensitivity of strain gage resistance to temperature is usually reported by the manufacturer through the temperature coefficient of resistance (TCR). The TCR for the semi-conductor strain gages used for this research was $15 \% / 100^{\circ} \mathrm{F}$. The output due to temperature for the metal foil strain gages was not linear, but the manufacturer provided a fourth order polynomial to determine the amount of output at a given temperature. For instance, at $100^{\circ} \mathrm{F}$, a single strain gage would give an output equivalent to an apparent strain of -8.77 microstrain $(\mu \mathrm{m} / \mathrm{m})$, which would produce an output of about $-22 \mathrm{mV}$ in a quarter-bridge arrangement with an excitation of 5V and a gain of 1000 .

Another temperature-related error source is apparent strain caused by different thermal expansion rates of the strain gage and the test specimen material. In this case, a strain gage bonded to an unloaded specimen could be put into tension, and therefore give an output, if the test specimen expands thermally at a rate greater than the strain gage.

Temperature can also affect the sensitivity of the strain gage. The change in gage factor due to temperature of metallic strain gages is very small, but for semi-conductor strain gages this is much more of a concern. For instance, the temperature coefficient of gage factor (TCGF) of the metal foil strain gages was $0.778 \% / 100^{\circ} \mathrm{F}$, while the TCGF of the semi-conductor strain gages was $-13 \% / 100^{\circ} \mathrm{F}$. This indicates that as temperature increases, the gage factor of semi-conductor strain gages decreases significantly.

There are methods for attempting to compensate for these temperature effects. Using identical strain gages in adjacent arms of the Wheatstone bridge, such as in either the half- or full-bridge configurations used in this work, and ensuring that the strain gages 
are at the same temperature can compensate for the resistance change due to temperature. In this case the thermal output for the strain gages should be the same, and therefore cancel out in the bridge. Apparent strain due to thermal expansion can also be accounted for in this manner if the strain gages are bonded to the test specimen in a manner such that they both undergo the same thermal expansion load. In addition, the bending halfand full-Wheatstone bridges described earlier are theoretically insensitive to axial loads, which would include thermal expansion loads. Also, metal foil strain gages can be obtained with backing material with matched thermal expansion coefficients for different materials, theoretically eliminating loads due to different thermal expansion rates. The foil strain gages used for the final skin friction sensor used strain gages with thermal expansion coefficient matched to the aluminum to which they were mounted (the epoxy used to bond the strain gage to the substrate is not a factor if applied very thin, as specified). It is more difficult to account for the change in gage factor experienced by the semi-conductor strain gages. One attempt to do this makes use of a special resistor in series with the input voltage to the Wheatstone bridge, which effectively the lowers the voltage applied to the Wheatstone bridge. As temperature increases, and the gage factor of the strain gages decreases, the resistance of the series resistor goes down, increasing the voltage across the Wheatstone bridge. By selecting the resistor carefully, it is possible to cancel the effect of the dropping gage factor with the increase in excitation voltage, but only for a limited temperature range $\mathrm{e}^{36}$.

These methods are used widely, although they only work perfectly in the theoretical case. Since no two strain gages have exactly the same properties, it is difficult to completely eliminate temperature effects in practice using the cancellation methods above. This is especially true for semi-conductor strain gages, due to the complex doping method by which they are made ${ }^{35}$. Metal foil strain gages, however, are much more uniform gage to gage, and these methods therefore work better for them.

The trade-off between the two types of strain gages now becomes obvious. The semi-conductor strain gages, with their extreme sensitivity, allowed the design of cantilevered beam skin friction sensors with very small deflections. However, to avoid thermal errors due to the high temperature sensitivity, it was necessary to incorporate active water-cooling to maintain constant temperature of the strain gages, especially 
considering the hot flow environment. The water-cooling systems made the first two skin friction sensors difficult to integrate into the free-jet wind tunnels in which they were tested, and led to other difficulties that will be discussed later. The difficulty in thermally compensating these strain gages precluded them from being used on the flight vehicle skin friction sensor, since the water-cooling system was not available on the flight vehicle. The foil strain gages, though they have a much lower gage factor, were therefore chosen for their superior thermal characteristics. 


\section{CHAPTER THREE:}

\section{TEST APPARATUS AND INSTRUMENTATION}

\subsection{Electronics}

In order to provide the necessary support for the strain gage based sensors used in this study, special signal conditioning amplifiers were used. These units, Micro Measurements Model 2310, combine several different functions into one device. These functions include excitation voltage (with a range from 0.7 to 14 DC Volts), signal amplification (with adjustable gain from 1 to 11,000), signal filtering (low-pass, 2-pole Butterworth, with cut-off frequencies of 10,100,1000, or 10,000 Hz), and bridge completion and balancing. The bridge completion feature allows the same unit to be used for quarter-, half-, or full-Wheatstone bridge configurations by wiring the strain gage leads to different pins of the connector. The auto-balancing feature makes it possible to balance, or bring to zero, the output of the bridge, which is useful in eliminating a fixed offset voltage. A high input impedance of over 100 megohms prevents the unit from affecting the output signal.

These signal-conditioning amplifiers were used during testing of the skin friction sensors developed in this study. This included the various calibrations, cold-flow wind tunnel tests, and scramjet wind tunnel tests. Since all of the skin friction sensors were designed for the same or similar flow environments and wall shear levels, they were designed to produce about the same amount of output. Therefore, the same settings were used on the signal conditioning amplifiers for almost all of the tests. An excitation voltage of 5 volts with a gain of 1000 was applied to the Wheatstone bridges of the first two sensor designs. Although higher excitation voltages were technically possible, it was found that using an excitation of 10 volts caused excessive drift of the semiconductor strain gages due to self heating. Therefore, the above stated settings were used tests of the skin friction sensors with the semi-conductor strain gages. Since the foil strain gages were not as temperature sensitive, a higher excitation voltage was possible. As seen in Equations 14 and 15, output voltage scales directly with excitation voltage. So using a 
higher excitation produces a larger output, which improves the signal to noise ratio. Therefore the third skin friction sensor used an excitation voltage of 10 volts and a gain of 500 .

Other various electronic instruments were used in the acquiring of data during the different tests. Discussion of these instruments will be presented as relevant to the description of those tests.

\subsection{Calibration}

Direct measuring skin friction sensors have the distinct advantage of being simple to calibrate. Any method of applying known forces to the surface of the floating element can, in theory, be used. Two such methods were used in this work. The first of these was a simple, standard static calibration. The second made use of a glycerin flow with a known shear level in order to provide a dynamic calibration.

\subsubsection{Static Calibration}

A static calibration was performed on all of the skin friction sensors in this study. The method used is illustrated in Figure 14. The method was a direct force application method. The gage was clamped in a horizontal position, and a cone was hung from the flow surface of the floating head with string and tape. Excitation of the strain gages was provided by the signal conditioning amplifiers, and the bridge output was balanced (trimmed to zero). Calibrated masses were then placed in the cone, and the

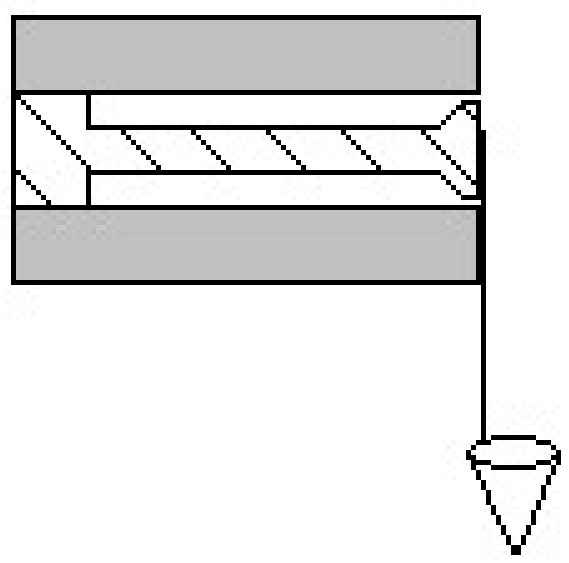

Figure 14: Static Calibration Method 
corresponding output was read from a multimeter and recorded. Careful attention was given to ensure that the hanging weight was aligned with the desired axis of the skin friction sensor. This process was repeated to ensure repeatability of the sensor. Both positive and negative directions were calibrated along the sensing axis. For the first two designs, which incorporated two orthogonal sensing axes, the off-axis output was also recorded.

The input mass was converted to a force by simply multiplying by the gravitational constant $(\mathrm{F}=\mathrm{m} \cdot \mathrm{g})$. The force was then converted to a shear by dividing by the area of the flow surface of the floating head $\left(\tau_{\mathrm{w}}=\mathrm{F} / \mathrm{A}_{\text {head }}\right)$. This produced calibration curves that directly related the skin friction sensor output voltage to the shear acting on the floating head. Results of the calibration tests will be presented and discussed for the different designs in their respective chapters.

\subsubsection{Dynamic Calibration}

The dynamic calibration method made use of the Virginia Tech skin friction calibration rig, shown in Figure 15. This rig establishes a fully-developed channel flow between two flat plates, where the shear is directly related to the pressure drop. Pressure transducers were used to measure the channel pressure drop, which is linear with distance, and the wall shear can then be found. A vertical storage tank contains the working fluid, which was glycerin. By varying the height of the fluid in the storage tank, different pressure drops, and therefore different shear levels, can be achieved. An overflow outlet is used so that flow from the reservoir will maintain a constant height at the top of the storage tank as the flow through the channel is opened and closed. This then maintains a constant pressure drop and shear level. By shutting off the flow from the reservoir to the storage tank, it is possible to do unsteady calibrations as the fluid height in the storage tank drops, causing falling shear levels.

The dynamic calibration was only used with the first and second skin friction sensor designs. These sensors were mounted flush with the surface of the lower channel wall. Excitation and balance was provided by the 2310 signal conditioning amplifiers. The storage tank was filled from the reservoir to a predetermined level. The flow valve at the end of the channel was then opened, and flow was established through the channel. During the testing, data was recorded on a National Instruments DAQ System Model 


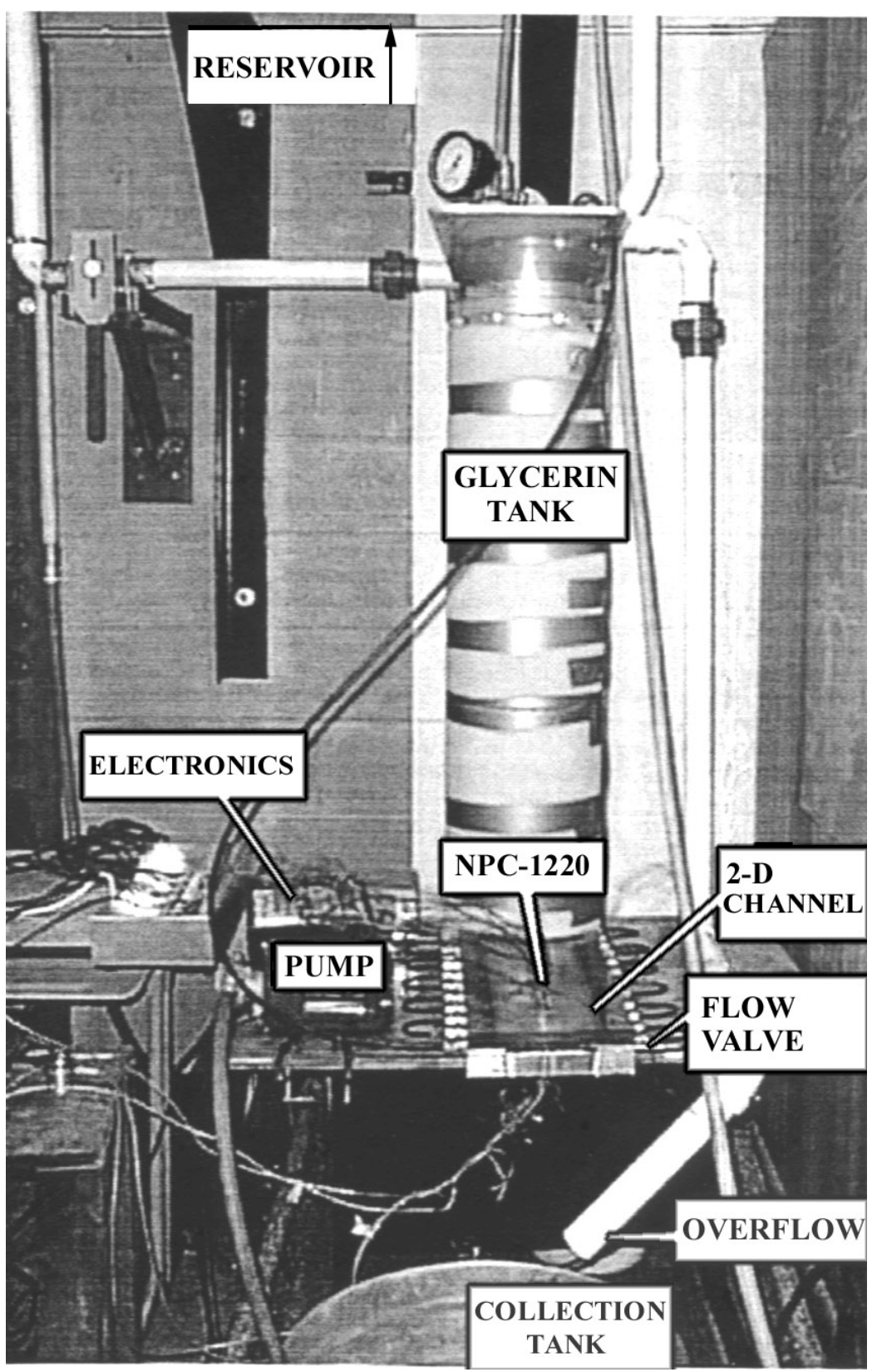

Figure 15: Calibration Rig

Number 2090 on a PC using LabView data acquisition (DAQ) software. Again, the results of these calibrations will be presented in the discussion of the relevant gage design.

\subsection{Vibration Tests}

The different designs of this study were subjected to modal analysis and shaking tests to determine their natural frequencies, frequency response functions, and overall ability to withstand vibrational environments. For these investigations, two different 
types of tests were done: impact hammer tests, and shaking tests. For both types of tests, the skin friction sensors were electronically excited and balanced with the 2310 signal conditioning amplifiers. Also, for all tests, the output was recorded and processed with a Hewlett-Packard 35665A Dynamic Signal Analyzer (DSA).

For the first of these tests, an impact hammer was connected to the DSA. By hitting the skin friction sensor with the impact hammer, the sensor was vibrationally excited over the entire spectrum of frequencies. The DSA recorded the output from the sensor and the hammer and performed a Fast-Fourier Transform (FFT) to reveal the response of the skin friction sensor over the frequency spectrum. These tests were only conducted on the first skin friction sensor design, and the results will be presented in the relevant later section.

The second type of tests that were done made use of dynamic shakers (made by Ling Dynamic Systems, LTD and Vibration Test Systems) to excite the skin friction sensors at controlled frequencies and levels. A diagram of the setup is shown in Figure 16. The DSA provided the source signal, which could be specified for fixed sine waves, sine wave frequency sweeps, random noise, or other types of input. This signal was amplified by a gain of about 30 by routing it through an Onkyo Integra M-504 Stereo Power Amplifier. From the amplifier the signal was sent to the shaker. In simple terms, the shaker contains a coil through which the current from the signal is sent. In the center

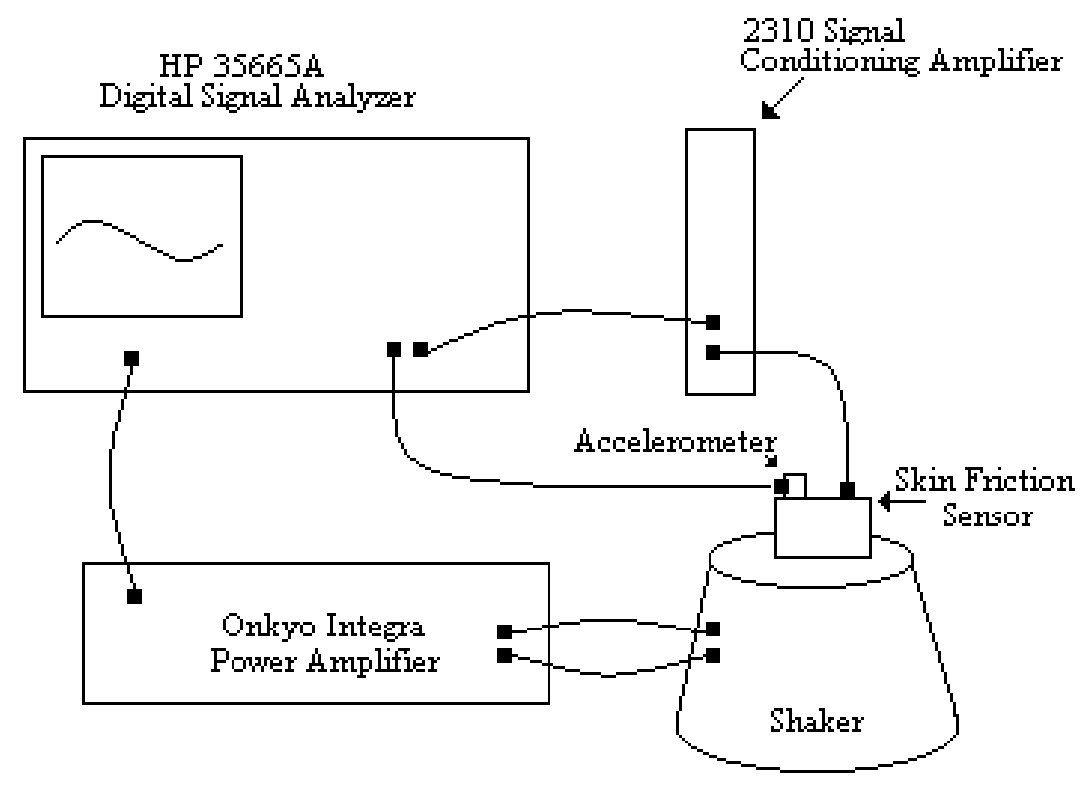

Figure 16: Shaking Test Setup 
of the coil is a metal rod, which then moves under the solenoidal force created by the current in the coil. The rod has mounting screw holes so that structures may be physically connected to the shaker. In this way, the motion of the rod was transmitted to the skin friction sensor. An accelerometer was mounted to the structure to determine the level of the input. The accelerometer gives an output that is directly proportional to the acceleration; the calibration is given in terms of volts per $\mathrm{g}$.

\subsection{Virginia Tech 9-inch x 9-inch Supersonic Wind Tunnel}

All of the skin friction sensors designed for this study were tested in a simple, cold supersonic flow before being tested in the complicated, high enthalpy flow of the scramjet engines. This was done using the Virginia Tech 9-inch by 9-inch supersonic blow-down wind tunnel. This facility provided a well-understood flow to further validate the sensor design results. The boundary layer characteristics of the tunnel floor plate have been previously measured, allowing the skin friction coefficient to be calculated using the Van Driest compressible correction to the incompressible turbulent integral method of Moses. These tests also demonstrated the versatility of the designs, being able to operate in more than one type of facility ${ }^{38}$.

A schematic of the facility is shown in Figure 17. This wind tunnel operates in the blow-down mode, meaning that air stored in high pressure tanks is allowed to flow through the wind tunnel before entering a diffuser leading to the atmosphere. An Ingersoll-Rand Type 4-HHE-4 4-stage reciprocating air compressor is driven by a 500 hp, 480V Marathon Electric Co. motor in order to provide the high-pressure air. With this compressor the storage system can reach pressures up to $51 \mathrm{~atm}$. A drying and filtering system is provided which includes both drying by cooling and drying by absorption. By changing nozzle and test sections, the facility can operate at Mach numbers of 2.4, 3.0, and 4.0. Reynolds number per meter for the wind tunnel ranges from $2 \times 10^{6}$ to $5 \times 10^{6}$. The test section walls have optical quality glass windows, allowing Schlieren and shadowgraph photography. The flow is dampened through the use of several screens and a perforated cone in the settling chamber ${ }^{39}$. A fast-acting butterfly valve in series with a hydraulic servo-valve is used for pressure regulation ${ }^{35}$. A pressure transducer and thermocouple in the settling chamber monitor the total pressure and temperature, respectively. Custom LabView software on a PC reads the total 


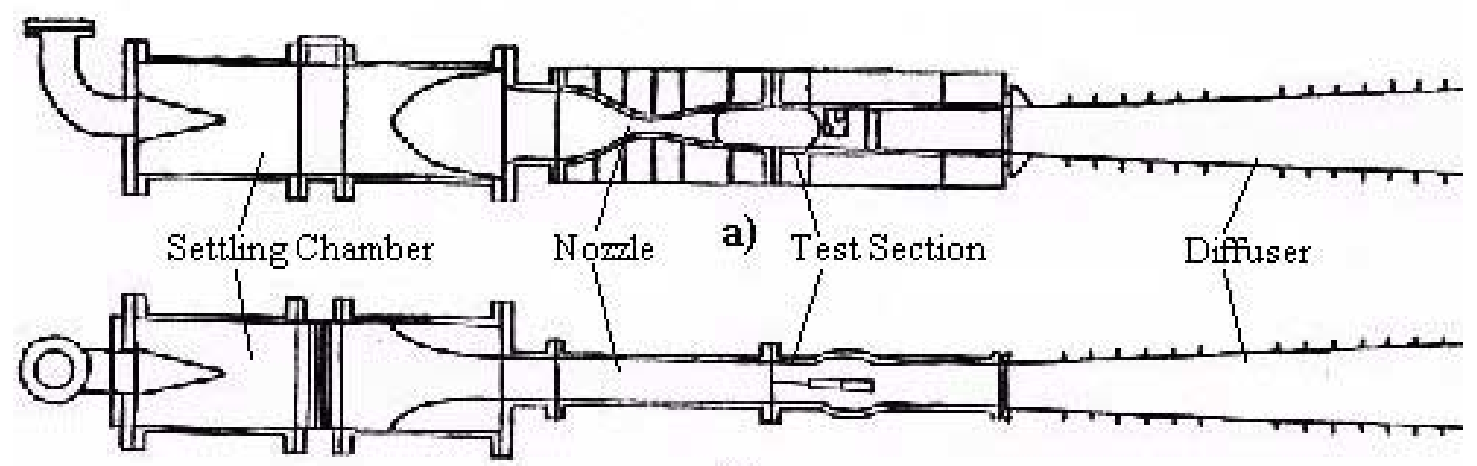

b)

Figure 17: Virginia Tech 9-in. x 9-in. Supersonic Wind Tunnel a) Side View b) Top View ${ }^{39}$ pressure and provides both feedback control of the tunnel and data acquisition. Analog to digital conversion is done with a 12-bit National Instruments A/D card.

For the skin friction tests, nominal tunnel conditions were Mach 2.4, with stagnation pressure, $\mathrm{P}_{0}=50 \mathrm{psia}(345 \mathrm{kPa})$, and stagnation temperature, $\mathrm{T}_{0}=540{ }^{\circ} \mathrm{R}(300$ $\left.{ }^{\circ} \mathrm{K}\right)$. Run times were typically five to ten seconds long. The skin friction gages were located in a section of the flat wall just downstream of the diverging section. Data was recorded at $100 \mathrm{~Hz}$ with filtering at $10 \mathrm{~Hz}$ using the filter built into the 2310 signalconditioning amplifier.

These tests provided validation of the skin friction gages. Gages that performed well in this facility also performed well in the scramjet tests, while a design that did not perform well in this facility also failed to perform well in the scramjet flow environment. The results of the cold supersonic tests of each specific gage will be presented in the corresponding chapter. 


\section{CHAPTER FOUR:}

\section{GAGE DESIGN ONE- RBCC TEST DESIGN}

\subsection{Gage Design}

The goal of the first skin friction sensor design was to begin the development of skin friction sensors for the Hyper-X scramjet engine. The design was influenced by and extended from the previous successful scramjet-tested skin friction sensors of DeTurris and Chadwick ${ }^{9,24,25,35}$. This design was then verified by testing in the combustor of a rocket-based-combined-cycle (RBCC) engine operating in scramjet mode.

\subsubsection{Gage Description}

The design is shown in schematic form in Figure 18. The floating head mounts to a stainless steel tube, which forms the flexible member for this design as a cantilever beam. Four semi-conductor strain gages are arranged in two half-Wheatstone bridges at the base, one for each axis of flow. Hypodermic tubes route water through the base and into the beam, where a setscrew blocks the flow just above the location of the strain gages. Additional 1/16-inch $(0.159 \mathrm{~cm})$ copper tubes route water around the exterior of the housing. The cavity between the beam and the housing is filled with silicon oil ${ }^{38}$.

The floating head is one-inch thick copper, with a diameter at the surface of 0.300 inches $(0.762 \mathrm{~cm})$, tapering to 0.290 inches $(0.737 \mathrm{~cm})$ at a distance of 0.030 inches $(0.0762 \mathrm{~cm})$ from the surface. The material and thickness of the head were chosen to match the properties of the surrounding engine wall as closely as possible. The bottom of the head was drilled and tapped for \#5-40 threads so that the floating head could be screw-fastened to the beam.

The floating head was mounted to the flexible member, which in this case was a cantilever beam made of stainless steel tubing. The outer diameter was 0.1575 inch $(0.400 \mathrm{~cm})$, and the inner diameter was 0.1024 inch $(0.260 \mathrm{~cm})$. The length of the beam was 2.250 inch $(5.715 \mathrm{~cm})$, with the bottom 0.250 inch $(0.635 \mathrm{~cm})$ sunken into the brass base of the sensor. Flats were machined into the outer diameter of the beam along the 0.250 inches $(0.635 \mathrm{~cm})$ above the base, such that four orthogonal faces were made. The 


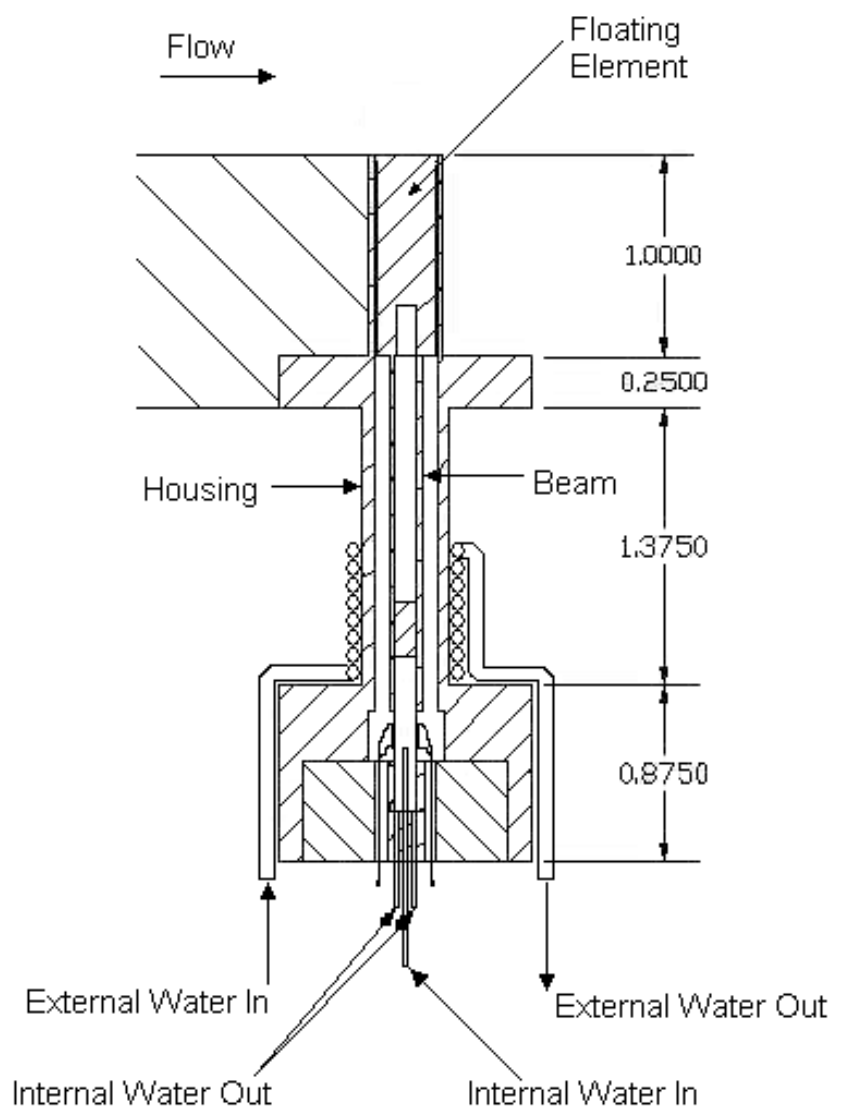

Figure 18: Gage Design One Schematic

strain gages were mounted on these faces, and the faces were aligned parallel and perpendicular to the flow direction, so that both a stream-wise and cross-stream component of wall shear could be measured. These flats can just be seen at the base of the beams in Figure 19.

Stainless steel tubing was selected for the beam for several reasons. First, it is a high-precision product readily available from tubing manufacturers. Second, the relatively low (compared to copper) thermal conductivity provides some thermal isolation of the strain gages. Thirdly, the interior of the tubing could be drilled and tapped. This allowed a setscrew to be used as a plug to block the internal water flow from traveling to the top of the beam. Another setscrew was used to thread the floating element to the beam. Both setscrews were thread-locked with high temperature ceramic adhesive ${ }^{38}$.

High temperature ceramic adhesive was also used for bonding the beam into the brass base. Brass was chosen for the base to provide further thermal isolation and for its 
relative ease of machining. The base was cylindrical, with a diameter of 1.000 inch (2.54 $\mathrm{cm})$ and a thickness of 0.500 inch $(1.27 \mathrm{~cm})$. A blind hole was drilled 0.250 inch $(0.635$ $\mathrm{cm}$ ) deep into the upper surface, and the lower portion of the beam was inserted into this hole. Five holes of 0.0625 -inch $(0.159 \mathrm{~cm})$ diameter were drilled through the base; four of these were for the strain gage wire leads, and one was for an oil fill tube. Two clearance holes for \#4-40 screws were drilled to allow the base to be mounted to the housing. In the center of the base, three tiny holes were drilled for the internal cooling lines. The central hole had diameter of 0.028 inch $(0.0711 \mathrm{~cm})$, and the two side holes had diameter of $0.024 \mathrm{inch}(0.0610 \mathrm{~cm})$. These holes accommodated hypodermic tubes used to route cooling water into and out of the stainless steel beam. An O-ring groove (not shown) was also incorporated to provide a sealing mechanism ${ }^{38}$.

The entire floating element-beam-base assembly was contained in a copper housing. The housing was also made of copper since it was to be inserted into the engine wall. The upper portion of the housing consisted of a one-inch sleeve with outer diameter of 0.364 inch $(0.925 \mathrm{~cm})$ above a 0.250 -inch $(0.635 \mathrm{~cm})$ oval-shaped thick flange with clearance holes for \#1/4-20 mounting screws. This allowed the housing to be mounted into the same mounting holes used for heat flux sensors, as described earlier. An O-ring was fitted over the sleeve and down to the flange. This O-ring fit into a groove that was designed into the heat flux sensor mounting holes, sealing the gage into the engine. The internal diameter of the housing was 0.310 inch $(0.787 \mathrm{~cm})$, which gave the floating head a gap of 0.005 inch $(0.0127 \mathrm{~cm})$ on each side at the surface. Below the flange, the housing maintained an outer diameter of 0.430 inch $(1.092 \mathrm{~cm})$ for 1.375 inch $(3.493$ $\mathrm{cm})$, before expanding to 1.250 inch $(3.175 \mathrm{~cm})$ to accommodate the brass base. Mounting holes for \#4-40 screws were drilled and tapped inside this portion of the housing so that the brass base could be fastened to it.

Hypodermic tubes routed through the base of the sensor provided the internal cooling. One tube, with outer diameter of 0.028 inch $(0.0711 \mathrm{~cm})$ and inner diameter of 0.020 inch $(0.0508 \mathrm{~cm})$, was routed through the center of the base and a short distance into the internal cavity inside the stainless steel beam. By using this tube as the inlet for the internal cooling water, the cooling water was directed to the location on the inside of the beam directly opposite to the location of the strain gages. The other two tubes, 


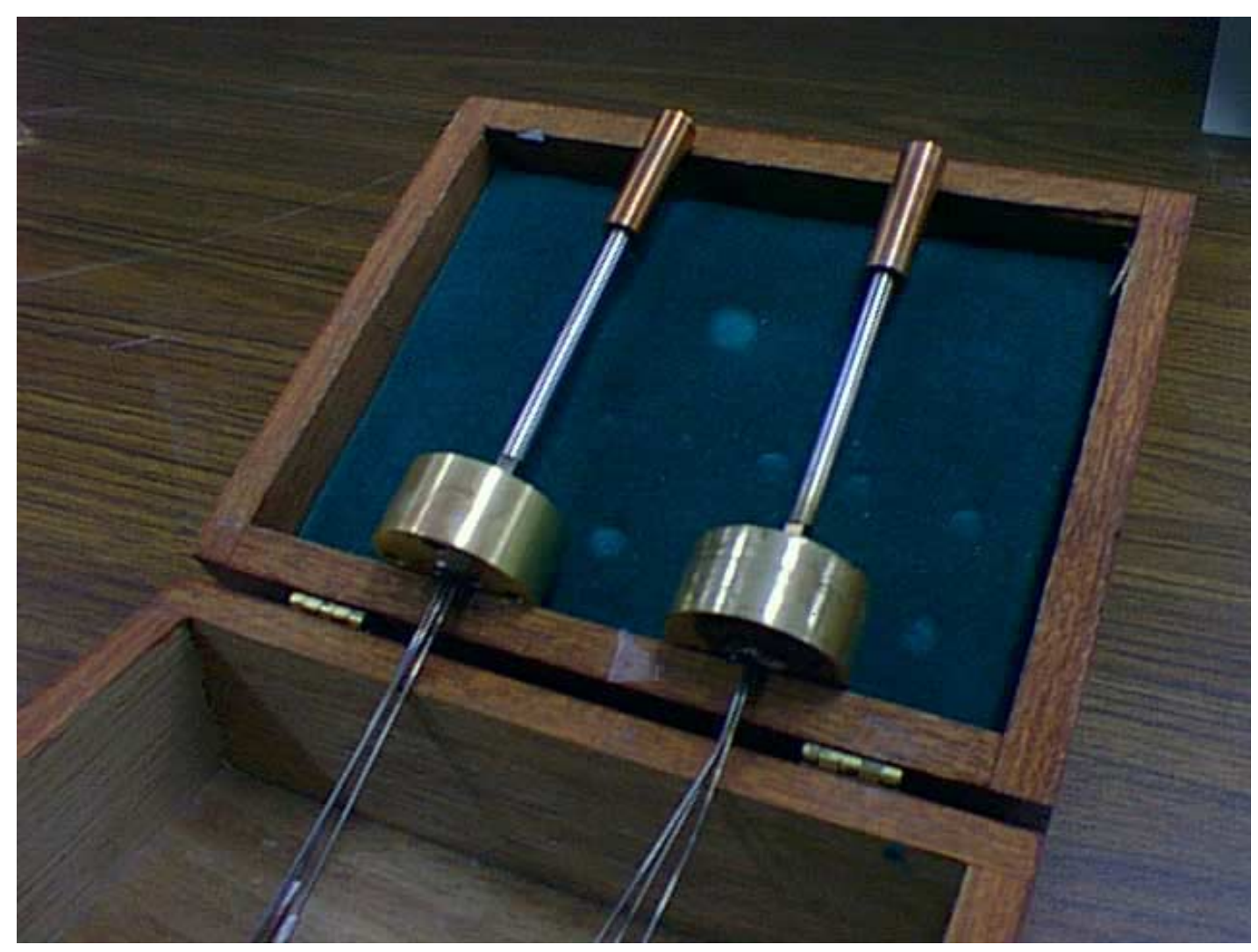

Figure 19: Design One Floating Element, Beam, Base, and Hypodermic Tubes

located on either side of the first, had outer diameters of 0.024 inch $(0.0610 \mathrm{~cm})$ and inner diameters of $0.017 \mathrm{inch}(0.0432 \mathrm{~cm})$. These were used as the outlets for the internal cooling water. Figure 19 shows the hypodermic tubes. Water-cooling was also applied to the exterior of the skin friction sensor by means of $1 / 16$ inch $(0.159 \mathrm{~cm})$ copper tubing coiled around the housing (see Figure 20).

This water-cooling was necessary to protect the sensor from the high temperatures caused by the high enthalpy flow, and to maintain a constant temperature of the strain gages. As was discussed earlier, semiconductor strain gages are temperature sensitive, and when using them in harsh temperature environments such as these, such measures are required to avoid errors. This is especially true when using semiconductor strain gages, such as were chosen for this design. The strain gages used in this application had a length of 0.060 in $(0.152 \mathrm{~cm})$ and a width of 0.008 in $(0.0203 \mathrm{~cm})$, with nominal gage factor and resistance of 140 and $540 \Omega$, respectively. The strain gages were obtained from Micron Instruments, who also performed the bonding and wiring of the strain gages.

This design was different from the designs of DeTurris and Chadwick. DeTurris ${ }^{9}$ 


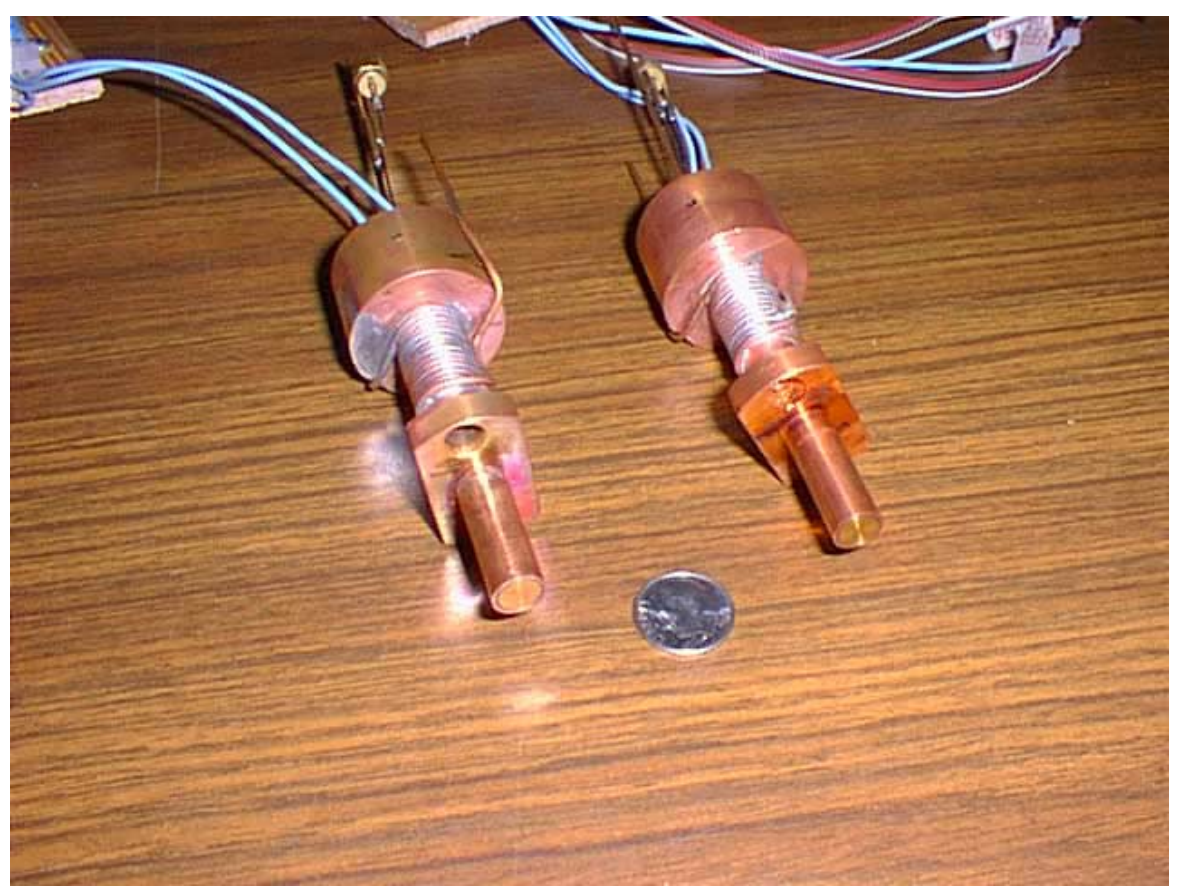

Figure 20: Design One Completed Gage

used a pre-manufactured strain gage assembly, and only routed cooling lines around the outer housing. Chadwick ${ }^{35}$ used semiconductor strain gages similar to those of this study, but he used a quartz beam. He also routed cooling lines internal to the quartz beam, but for a different purpose. His sensor was tested in even higher enthalpy flows, and the internal cooling lines of his design were meant to cool the backside of the floating head so that it would remain at a temperature close to that of the rest of the engine wall, instead of becoming a local hot spot. Therefore, his internal cooling line was routed to the near the top of the beam, creating an annular return flow. The current design, however, blocks the water flow inside the beam just above the height of the strain gage location, such that the most effective cooling occurs near the strain gages.

\subsubsection{Design Study}

A design study was performed to arrive at the beam geometry that was used. The floating head geometry was basically fixed by the necessity of fitting into the existing instrumentation holes in the engine wall, so the design variables were limited to the length and inner and outer diameters of the beam. The goal was to achieve a strain at the base of the beam large enough to produce a strong signal in the strain gages, while at the same time keeping the deflection of the head as small as possible. Using a spreadsheet with simple beam equations and inserting the dimensions of different available standard 
tubing sizes allowed an optimum to be found. For this design study, the entire beam was treated as if it had the same geometry as the tubing, i.e., the floating head was assumed to be rigidly attached to the end, and the geometry of the flats machined into the base of the beam were not modeled. For this situation, the moment of inertia for the stainless steel tubing is calculated from Equation 16,

$$
I=\frac{\pi}{64}\left(D_{o}^{4}-D_{i}^{4}\right)
$$

\section{Equation 16}

where $I$ is the area moment of inertia of the beam, $D_{o}$ is the outer diameter of the beam, and $\mathrm{D}_{\mathrm{i}}$ is the inner diameter of the beam. The strain is then calculated from Equation 9, with Equation 10 substituted for the stress, $\sigma$, yielding Equation 17:

$$
\varepsilon=\frac{P L c / 2}{E I}
$$

\section{Equation 17}

The force at the end of the beam, $\mathrm{P}$, is calculated by multiplying the expected shear level (units of force/area) by the area of the floating element surface exposed to the flow.

After the tubing for the beam was selected in this manner, a more detailed calculation was undertaken to verify the magnitude of the deflection of the floating element. This calculation followed the procedure outlined in Section 2.2, Equations 6 through 8. For this calculation, the moment of inertia and material properties of each section were considered. Hence, the moment of inertia of the section of beam where the flats were machined was calculated from Equation 18,

$$
I=\frac{s^{4}}{12}-\frac{\pi}{64} D_{i}^{4}
$$

\section{Equation 18}

where $s$ is the length of each side of the squared outer cross section, and $D_{i}$ is again the inner diameter of the tubing. The moment of inertia of the floating element, since it was solid, was calculated from Equation 19,

$$
I=\frac{\pi D^{4}}{64}
$$

\section{Equation 19}


where $\mathrm{D}$ is the diameter of the floating element. As stated in Section 2.2, Equation 8 was integrated twice to obtain the deflection at the end of each beam segment, starting at the base and moving to the end of the floating element. Boundary conditions were invoked at the ends of each segment to provide the necessary constants of integration. For the first segment, the boundary conditions at the base of the beam were zero deflection and zero slope since it was assumed to be rigidly clamped into the brass base of the sensor. The boundary conditions on the interior segments of the beam and floating element were such that continuity of slope and deflection were maintained. This calculation led to the result of an expected maximum deflection of about 0.0001 inch $(0.00025 \mathrm{~cm})$.

\subsubsection{Oil-Fill Advantages and Disadvantages}

Silicon oil (1000 cSt) was used to fill the interior space between the housing and the beam structure. Filling the interiors of skin friction sensors such as this with oil has long been the source of several benefits, and several difficulties. The oil provides thermal protection and stability to the interior of the sensor, and especially the strain gages, by providing a large thermal mass and by preventing the flow of hot gases from reaching the strain gages. The viscosity of the oil helps to dampen vibrations of the beam structure. The oil also protects against pressure gradient effects across the head by providing a level surface for the flow and preventing flow into and out of the gap surrounding the floating element.

There are also several difficulties related to the use of the oil to fill the gap of the skin friction sensor. The first issue is the difficulty of getting the oil into the interior space of the gage. Typically this is done through a long and tedious method, wherein the sensor is placed in a vacuum chamber with a reservoir of oil above the floating element. The chamber is evacuated, and the air inside the sensor slowly bubbles up through the reservoir of oil. After some amount of time, the chamber is bled back to atmospheric pressure, and the pressure difference across the reservoir and the interior of the sensor forces oil into the sensor through the gap around the floating element. Since the gap around the floating element is so small, it takes many cycles of this procedure to get all of the air bubbles out of, and all of the oil into, the sensor interior. And, it is very important to ensure that all of the air bubbles are removed. This is due to the fact that these sensors are tested at low static pressure, and a bubble that is small at ambient pressure would 
grow at low pressure and could put a force on the beam that is larger than the shear force due to the flow.

Another concern with the oil fill is the sealing of the sensor from oil leaks. This sensor proved to be difficult to completely seal. After filling one of these sensors with oil and placing in an upright position such that no oil could spill out of the gap around the floating element, the oil level would still decrease due to slow leaks through the brass base of the sensor. The source of these leaks was the holes in the base through which the strain gage leads were routed. Since these leads were coated with Teflon, it was difficult for epoxies to completely bond to them and provide a good seal. In addition, during testing oil from the sensor does not remain in the gap, but a small amount flows out and smears downstream along the flow surface. Whether or not this is a major cause of concern to the measurement of skin friction, the sight of oil smears on a scramjet engine is usually met with dissatisfaction on the part of the testing agency. In any case, both of these sources of oil loss require that the oil level of the sensor be monitored, and that the sensors be periodically removed and refilled with oil. Oil leaks also limit the possible orientation of the skin friction sensor. An ideal sensor could be used in any orientationright side up, upside down, sideways, etc. However, in any orientation other than right side up, oil filled sensors will have increased rates of oil loss. This very issue became very significant for design two, and led to an oil free design for design three.

\subsection{Calibration Results}

This sensor was calibrated statically as described in Section 3.2.1. It was not calibrated in the dynamic calibration rig because the rig was not in operating condition at the time. As described previously, the gage was clamped in a horizontal position, and weights were hung in alignment with one of the axes. Output was recorded from both the parallel and perpendicular Wheatstone bridge axes. The gage was rotated 90 degrees and the calibration was done on the next axis. This was done a total of four times, to calibrate each axis in both the positive and negative directions, and the whole process was repeated multiple times to ensure repeatability. A sample calibration curve for one of the sensors of this design is shown in Figure 21. 


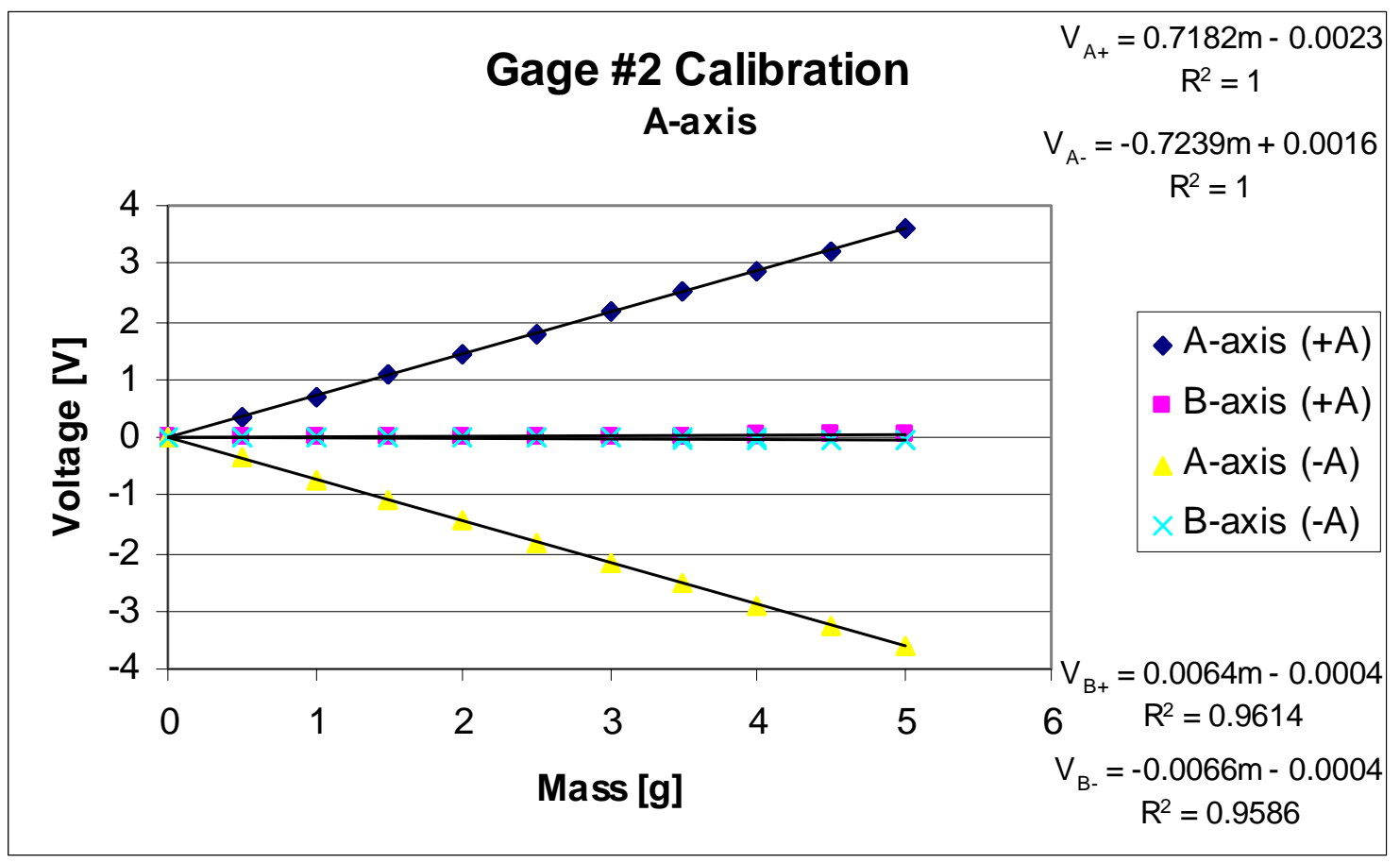

Figure 21: Sample Calibration For Design One

Figure 21 shows the results of calibration of an axis labeled " $A$ " in both the positive and negative directions. The legend labels the output according to the physical orientation of the sensor and the orientation of the loading. Hence the label " $\mathrm{B}$-axis $(+\mathrm{A})$ " indicates that the output shown is from the B-axis, which is perpendicular to the Aaxis, and the loading was aligned with the direction of positive output from the A-axis. Therefore, this output represents the amount of "cross talk," or output from one axis due to loading in the other axis. Theoretically, this should be zero, but due to imperfections in the machining of the flats and the bonding of the strain gages, the two axes are not perfectly orthogonal, leading to a small amount of such cross talk. It is clear from the chart that this cross talk is very small compared to the output from the intended axis.

The output was recorded for mass loading increments of 0.5 gram, from zero to 5 grams. In the upper and lower right hand corners of the chart are shown the results of linear fits to the calibration data points. The results include the slope and offset of the linear fit, as well as the $\mathrm{R}^{2}$ figure, which indicates how close to the linear fit the data points fall. An $\mathrm{R}^{2}$ equal to one indicates a very good fit. For the axis aligned with the load, linearity is quite good. The linearity of the off-axis output is not quite as good, but it is explainable. When the weight is hung from the floating head of the sensor, the 
weight at the end of the string usually swings slightly in a pendulum motion, and it is very difficult to completely arrest this motion. The semiconductor strain gages are so sensitive that even the small side-to-side force of this motion is detected by the off-axis, and hence creates a fluctuation in the output that is often of the same magnitude as the cross talk. Therefore the uncertainty in the cross talk output is much greater, leading to a poorer linear fit.

The linear fit shown in Figure 21 relates the output voltage to the mass hung from the head. As previously discussed, a relation between the output voltage and the shear on the head is obtained by first multiplying the mass by the gravitational constant, $\mathrm{g}$, to convert the mass to a force, and then dividing that force by the flow-exposed surface area of the floating element to obtain the shear stress (units of force/area). Therefore the shear can be found by multiplying the voltage by a single constant. For these gages, that conversion factor was typically about $750 \mathrm{~Pa} / \mathrm{V}$.

Calibrations were performed both before and after filling the housing with oil. Calibrating the skin friction gage with oil fill was challenging due to the necessity of using tape to adhere the string (by which the weight was hung) to the surface of the floating head. It became necessary to first use a cotton swab dipped in alcohol to wipe any oil from the floating head, and then to quickly apply the tape before more oil oozed onto the floating head surface. No difference was seen in the sensitivity of the sensor between the oil filled and oil free cases.

Calibrations were also performed with and without water flowing in the internal cooling channels. This was done in order to see if there was any output due to the water flow, and if the sensitivity of the sensor changed with temperature, since the water temperature was cooler (about $60^{\circ} \mathrm{F}$ ) than the ambient air temperature (about $75^{\circ} \mathrm{F}$ ). No output was seen due to the flow, and the sensitivity of the sensor was the same once a steady-state temperature was reached after turning on the flow. However, a substantial zero shift was seen in the output as the semiconductor strain gages adjusted to the change in temperature when the internal flow was turned on. This made it clear that in wind tunnel testing it would be necessary for the cooling water to be turned on long enough before the test window for the temperature of the strain gages to reach a steady value. 


\subsection{Vibration Tests}

This skin friction gage was also subjected to vibrational tests and modal analysis, as described in Section 3.3. First, impact hammer tests were conducted. For these tests, the skin friction sensor was struck with an impact hammer to excite the structure over the entire spectrum of frequencies, and the output from both the hammer and the sensor was recorded on the digital signal analyzer (DSA). The results of these tests made clear the natural and resonant frequencies of the skin friction sensor.

Typical results from one such test are shown in Figures 22 and 23. Figure 22 shows the magnitude ratio of the frequency response function (FRF), while Figure 23 shows the phase lag of the FRF. The magnitude ratio is simply a ratio of the voltages recorded by the DSA, and the numerical values therefore do not contain physical significance in the format shown. From the major peak on Figure 22 the natural frequency of the gage appears to be at about $32 \mathrm{~Hz}$. This relatively low natural frequency is due to the cantilevered beam structure with the massive floating element at the end. The natural frequencies can also be seen in the phase plot at the places at low frequencies

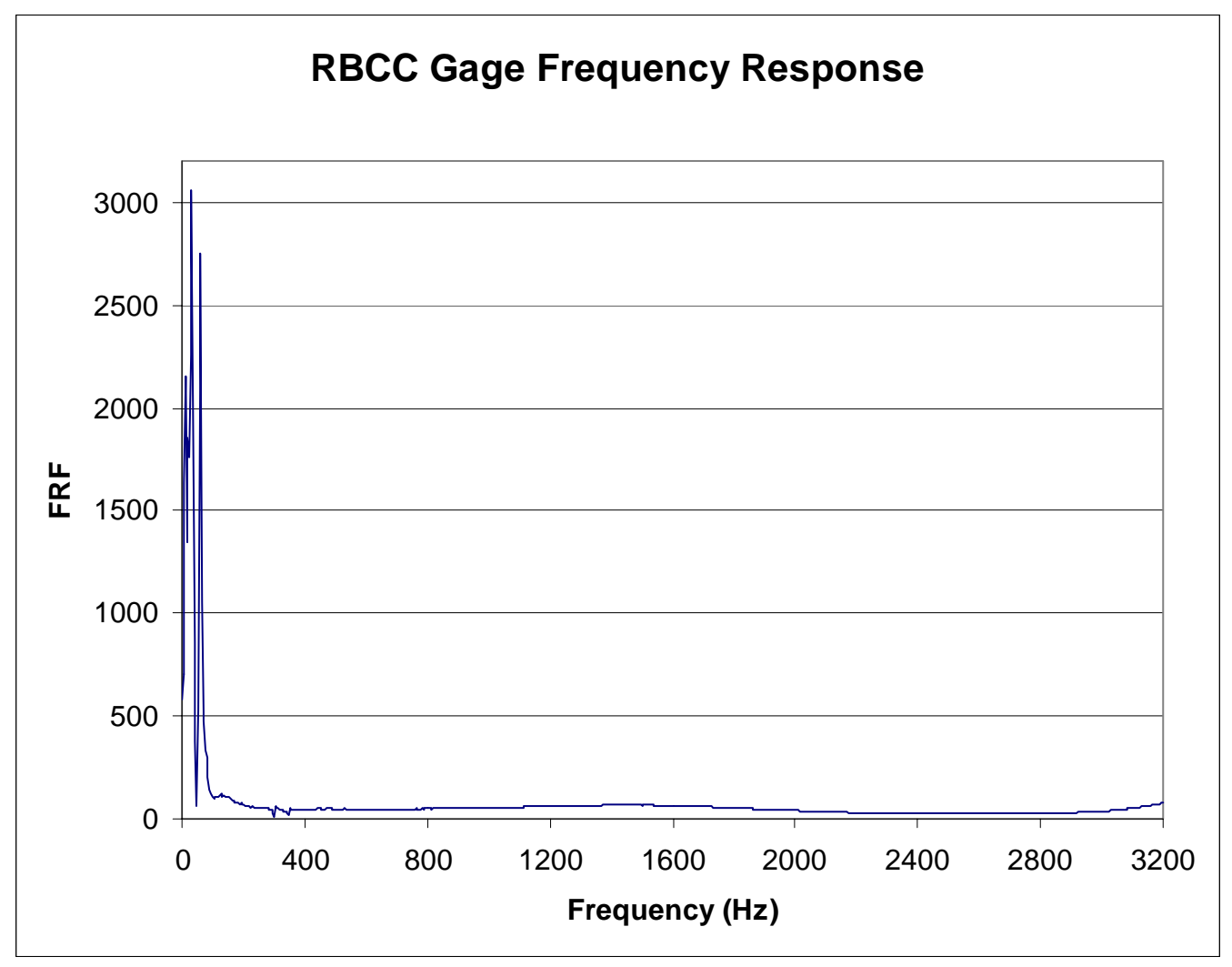

Figure 22: Hammer Test FRF (Magnitude) 


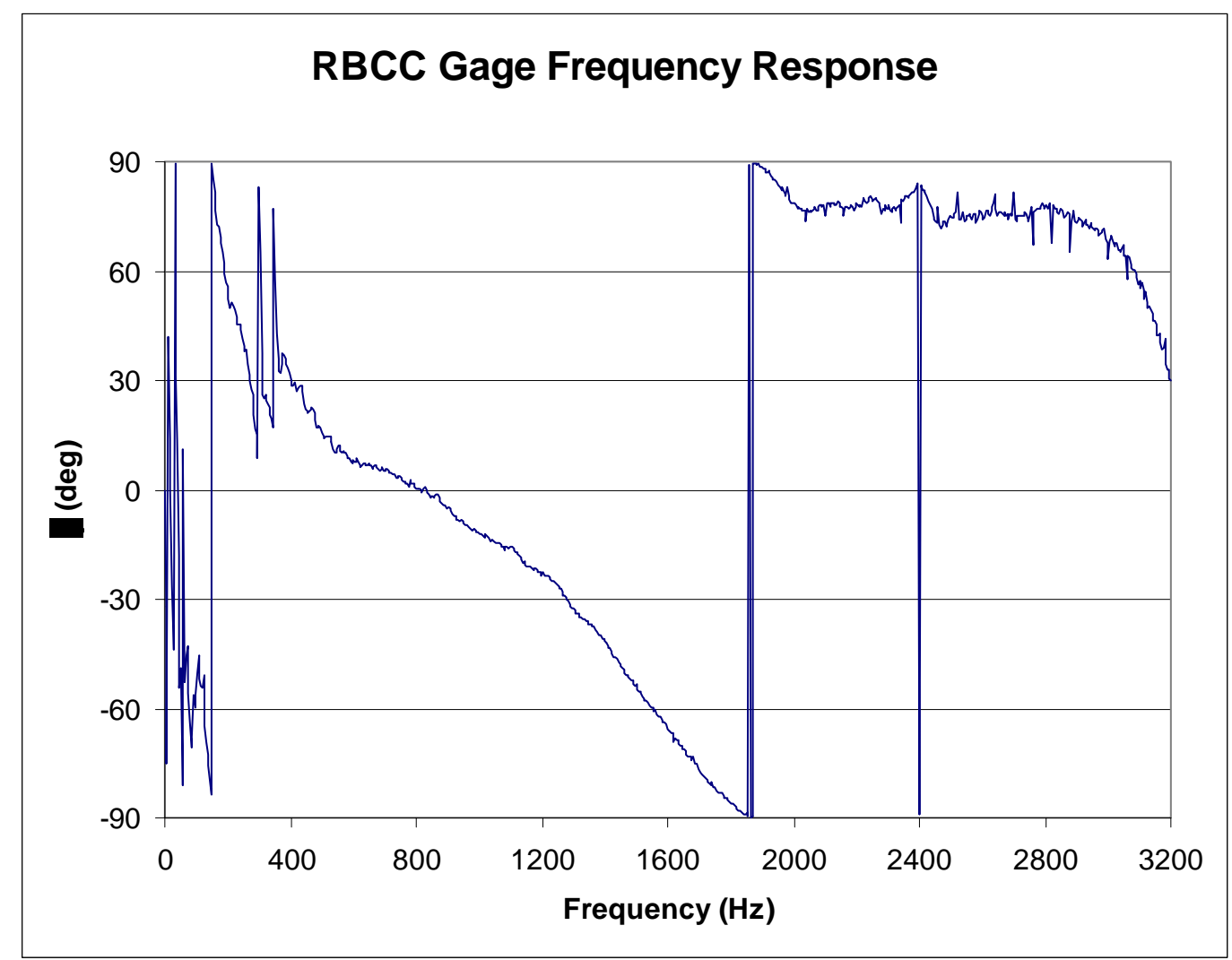

Figure 23: Hammer Test FRF (Phase)

where sudden spikes appear.

This skin friction design was also subjected to shaking tests. In these tests, a shaker, as described in Section 3.3, was used to vibrationally excite the sensor. The DSA supplied a random vibration signal over a frequency range of zero to $3200 \mathrm{~Hz}$. The Onkyo Integra amplifier amplified this signal before being sent to the shaker. A force transducer was connected in line with the shaking force between the shaker and the skin friction sensor housing, and the output was connected to the DSA. This allowed the output from the skin friction sensor to be related to the amplitude of vibration. Plotting this relation versus the frequency produces the FRF plots shown in Figure 24 and Figure 25. These plots indicate a natural frequency at about $40 \mathrm{~Hz}$, with a secondary mode at $272 \mathrm{~Hz}$. A coherence plot, shown in Figure 26, expresses the extent to which the signals from the force transducer and the skin friction sensor are related. Since the value for the coherence is close to one over the frequency range, the signals are closely related and the information from the other plots is meaningful. A power spectral density plot of the skin friction output produced by the DSA is shown in Figure 27. The power spectral density 
is another way of indicating the frequency content of a random signa ${ }^{36}$. The power spectral density (PSD) plot also shows the natural frequency to be at $40 \mathrm{~Hz}$, with the secondary frequency at $264 \mathrm{~Hz}$.

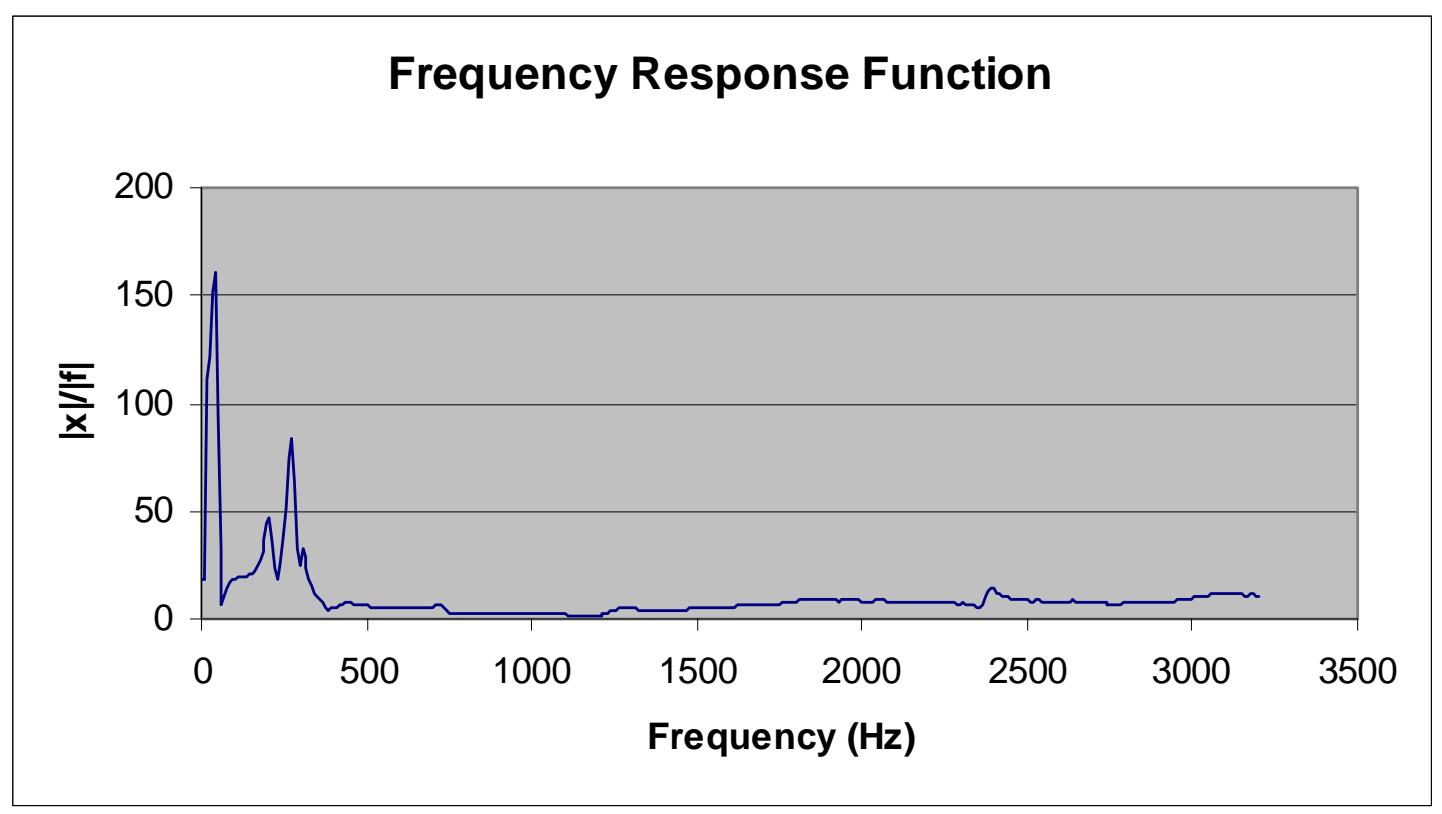

Figure 24: Shaking Test FRF (Magnitude)

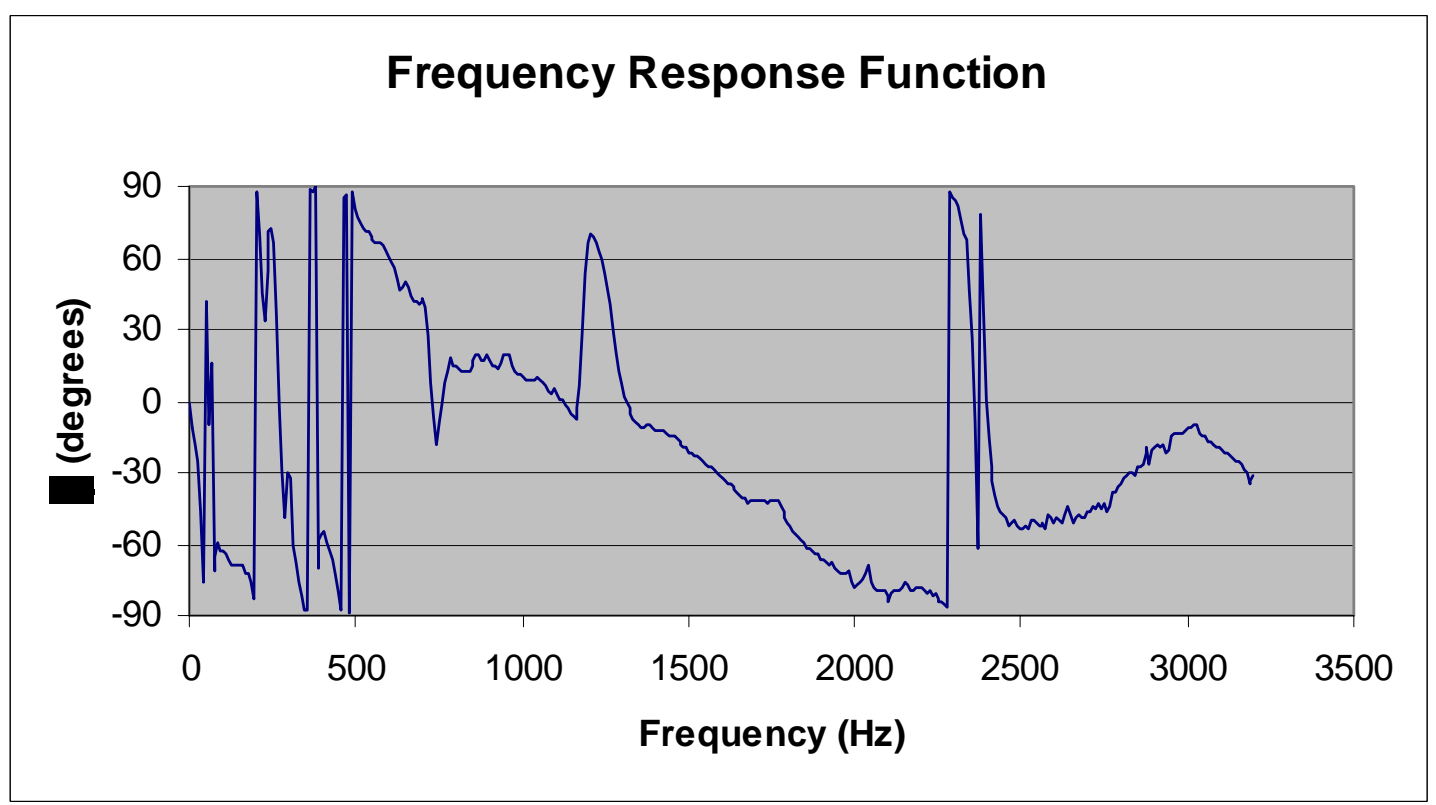

Figure 25: Shaking Test FRF (Phase) 


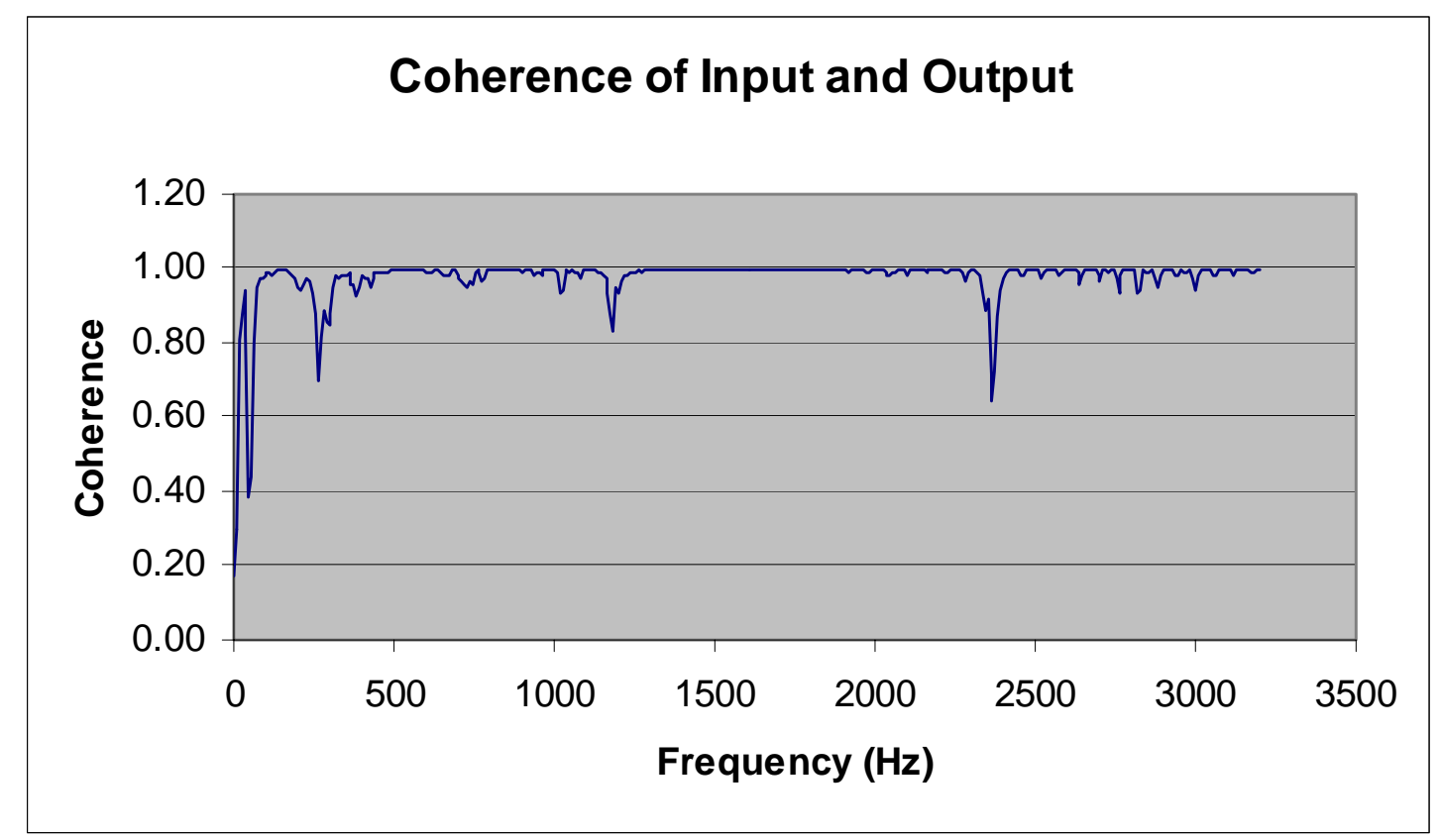

Figure 26: Shaking Tests Coherence

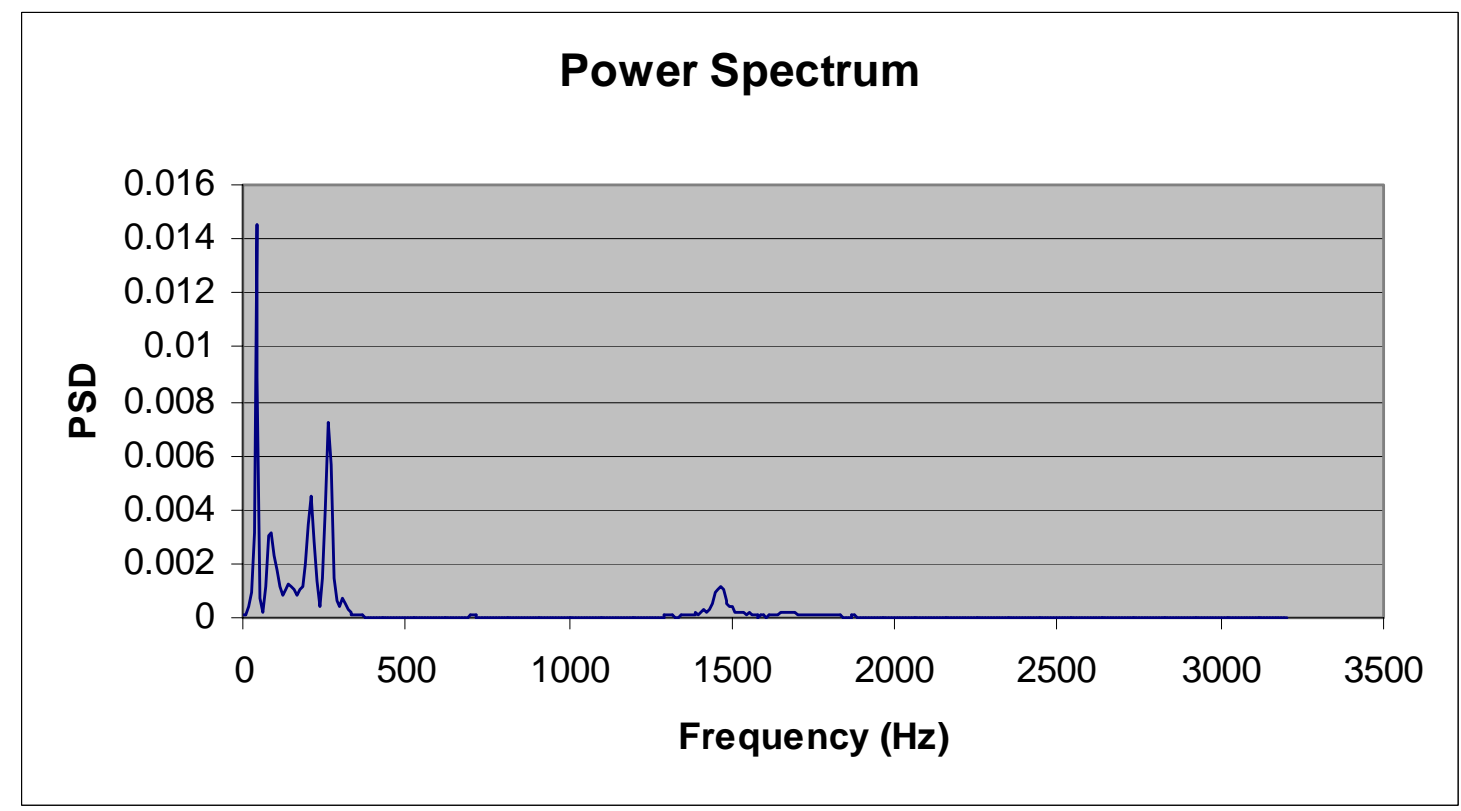

Figure 27: Shaking Tests Power Spectral Density 


\subsection{Cold Flow Tests}

To validate this sensor in a well documented supersonic flow environment, it was tested in the Virginia Tech 9-inch by 9-inch supersonic wind tunnel. These tests provided the first proof that the design would work in providing accurate measurements of skin friction in a supersonic flow. As stated in section 3.4, these tests were conducted at Mach 2.4, with stagnation quantities of $\mathrm{P}_{0}=50$ psia $(345 \mathrm{kPa})$ and $\mathrm{T}_{0}=540{ }^{\circ} \mathrm{R}(300$ $\left.{ }^{\circ} \mathrm{K}\right)$. The skin friction sensor was mounted in the flat aluminum tunnel floor plate, and provision was made so that the gage could be mounted with the flow aligned with either the positive or negative direction of either of the two axes. This allowed every possible orientation of the sensor with respect to the flow direction to be tested.

Cooling water flow to the external lines was not necessary for this cold-flow test. Cooling water was supplied to the internal lines at tap pressure (80 psi, $552 \mathrm{kPa})$ to provide thermal stability to the strain gages, though later tests proved that this also was not necessary. Data was recorded at $100 \mathrm{~Hz}$ on a PC via a 12-bit A/D card, and the signal was filtered at $10 \mathrm{~Hz}$ using the filter built into the 2310 signal conditioning amplifiers ${ }^{38}$. Static pressure was measured on the surface of the tunnel floor plate via a static pressure port located adjacent to the skin friction sensor. This measurement allowed the measured shear stress to be normalized by the local dynamic pressure, q, calculated from Equation 20.

$$
q=\frac{1}{2} \rho V^{2}=\frac{1}{2} \gamma P M^{2}
$$

\section{Equation 20}

In Equation 20, $\gamma$ is the ratio of specific heats for the test fluid, $\mathrm{P}$ is the local static pressure, and $\mathrm{M}$ is the local Mach number. For this case the test fluid is air, with $\gamma=1.4$, and $\mathrm{M}=2.4$. Normalizing the shear by the dynamic pressure produces the skin friction coefficient, $\mathrm{C}_{\mathrm{f}}$.

Two sensors of this design were built, and both were tested in the supersonic wind tunnel in multiple orientations during an extended series of tests. These two gages were aptly labeled Gage \#1 and Gage \#2. Gage \#1 performed somewhat better than Gage \#2 in the supersonic wind tunnel. The reason for this is unknown. Typical results from supersonic wind tunnel tests of Gage \#1 are shown in Figure 28 and Figure 29. 


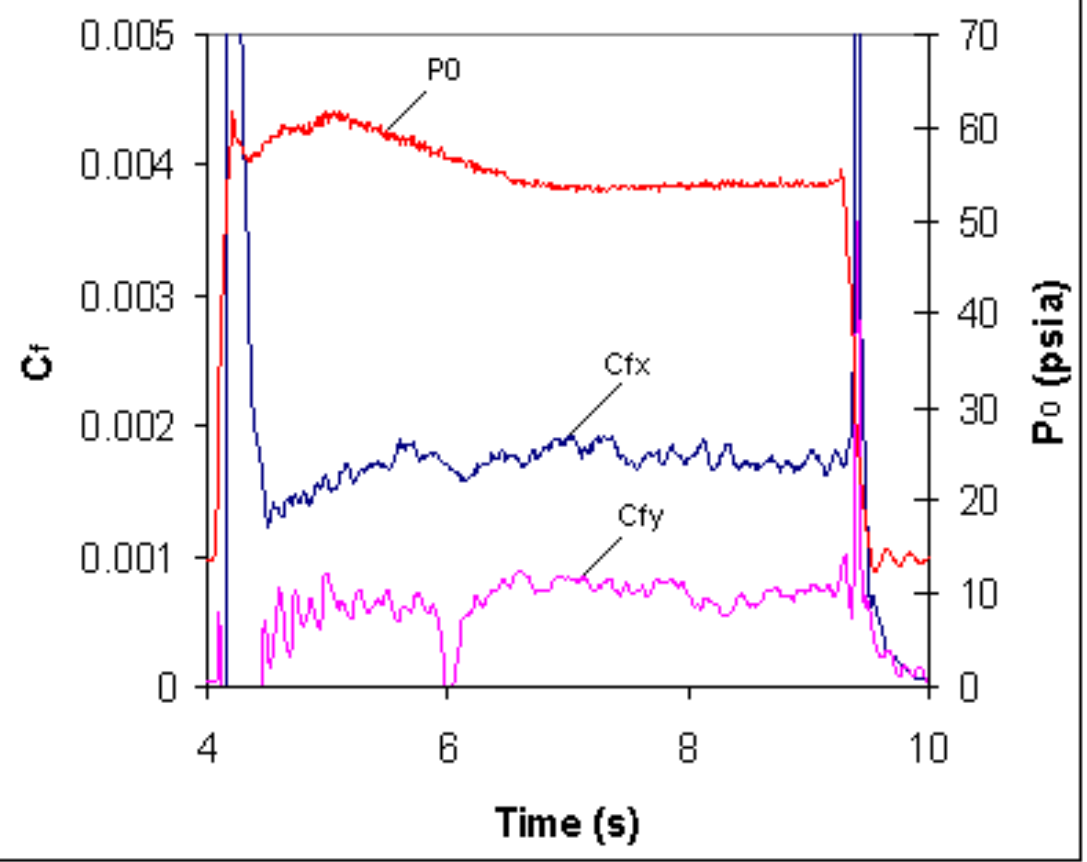

Figure 28: Supersonic Wind Tunnel Test Results: Run 3

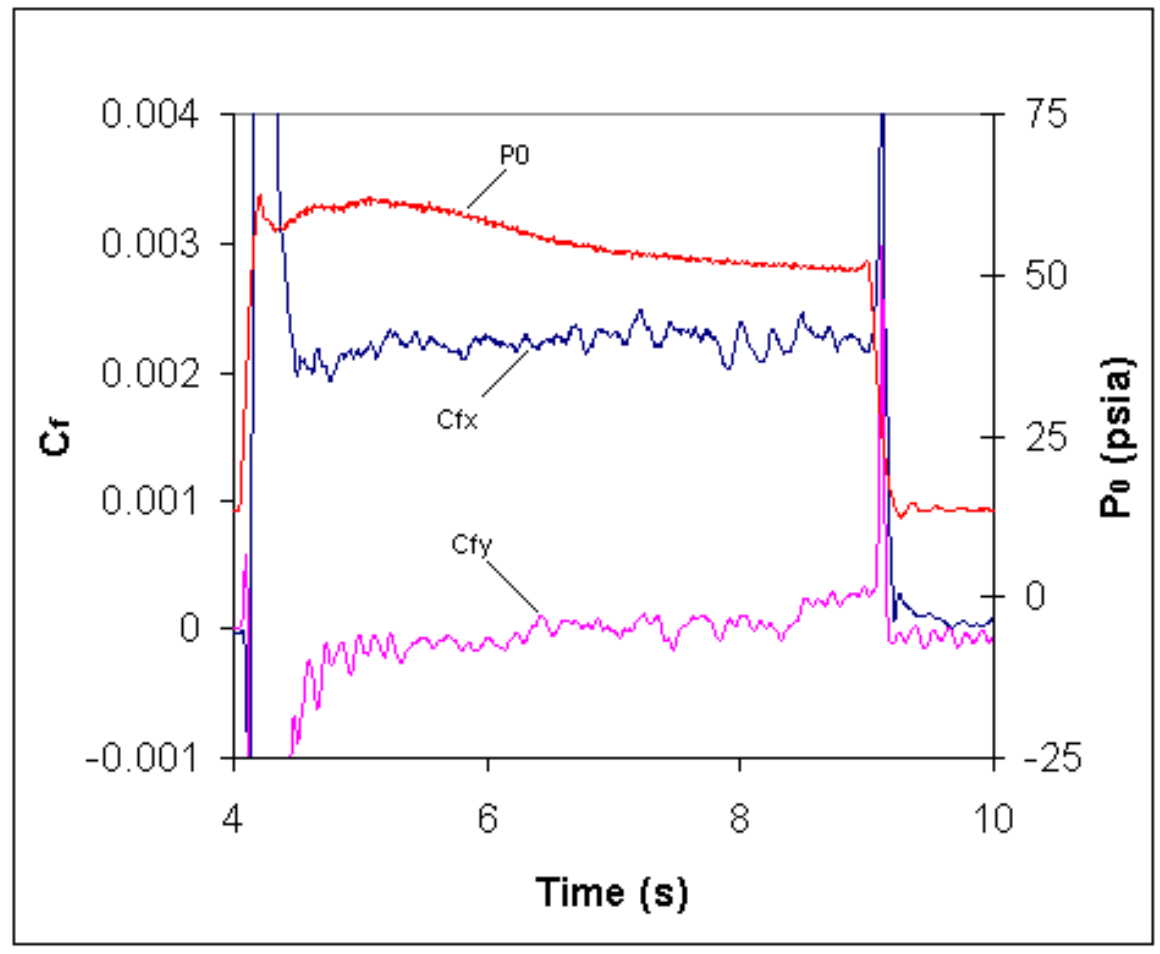

Figure 29: Supersonic Wind Tunnel Test Results: Run4 
In Figure 28 and Figure 29 the data curves labeled "Cfx" and "Cfy" refer to the stream-wise (X-direction) and cross-stream (Y-direction) components of the skin friction sensor output, respectively. These signals reflect the skin friction coefficient, $\mathrm{C}_{\mathrm{f}}$, meaning that the local dynamic pressure has been used to normalize the shear as described above. The data labeled "P0" refers to the total pressure of the wind tunnel. The total pressure shows timing of important events of the wind tunnel run, such as the starting and unstarting of flow through the nozzle. The large spikes in the skin friction output prove the fact that the output from the skin friction sensor clearly follows the starting and unstarting of the tunnel. This indicates good frequency response of the skin friction sensor, since the violent passing of shock waves and vibration causes these spikes. Another important feature of these data is the fact that the skin friction output starts at and returns to zero with a relatively constant level during the run. This shows that there is no zero-shift in the skin friction output due to thermal drift.

A summary of the output from these tests is recorded in Table 2. Here the average values of $\mathrm{C}_{\mathrm{f}}$ for the two components $\left(\mathrm{C}_{\mathrm{fx}}\right.$, and $\left.\mathrm{C}_{\mathrm{fy}}\right)$ are listed for each run. The resultant $\mathrm{C}_{\mathrm{f}}$ was calculated by taking the root of the sum of the squared values of the two components. $\mathrm{C}_{\mathrm{fx}}$ is the stream-wise component, and $\mathrm{C}_{\mathrm{fy}}$ is the cross-stream component. This flow is nominally 2-D, so the cross-stream component should be small. For the tunnel conditions described earlier, the Van Driest compressible correction to the integral method estimates a skin friction coefficient of 0.0018 to 0.0020 . The skin friction gage provided output within $10-15 \%$ of the estimated range of $\mathrm{C}_{\mathrm{f}}$. Some small differences

\begin{tabular}{|c|c|c|c|}
\hline Run & $\mathrm{C}_{\mathrm{fx}}$ & $\mathrm{C}_{\mathrm{fy}}$ & Calc. $\mathrm{C}_{\mathrm{f}}$ \\
\hline 3 & 0.00176 & 0.00069 & 0.00189 \\
\hline 4 & 0.00226 & 0.00003 & 0.00226 \\
\hline 5 & 0.00209 & 0.00089 & 0.00227 \\
\hline 6 & 0.00170 & 0.00089 & 0.00192 \\
\hline 7 & 0.00175 & 0.00099 & 0.00201 \\
\hline 8 & 0.00177 & 0.00094 & 0.00200 \\
\hline 9 & 0.00121 & 0.00124 & 0.00173 \\
\hline
\end{tabular}

Table 2: Supersonic Wind Tunnel Resuults Summary 
between the estimated and measured values are not surprising since the skin friction sensor was measuring at a location not far downstream from the end of the diverging section of the nozzle, where some thinning of the boundary layer due to the favorable pressure gradient could lead to higher skin friction values. Also, the mismatch of materials between the copper skin friction gage housing and floating element and the surrounding aluminum tunnel floor plate could cause a discontinuity in the heat flux, and hence the skin friction, at the sensor location. Taking these possibilities and the inherent measurement uncertainty into consideration (see Appendix A for repeatability of these tests), these results were quite encouraging, indicating that the skin friction gage design worked well in a cold supersonic flow environment, giving reasonable results.

The optical glass windows in the walls of the wind tunnel provided the opportunity to visually monitor the skin friction sensor during testing. It was particularly interesting to note the amount of oil that flowed from the gap of the sensor during each run. Small streaks of oil could be seen forming downstream of the gage. The amount of oil lost by a gage was found to be important after several runs. Tests conducted after a significant amount of oil had been lost showed less reliable results. This may be seen in the results summarized in Table 2, where Run 9 seems to indicate an equal amount of force in both the stream-wise and cross-stream directions. It is believed that once the oil level falls a certain amount, the output is worse than would be obtained if the housing were either completely full or completely empty. The oil remaining inside the housing may exert force on the beam and floating element as it is acted on by the flow and as it continues to flow out of the gap, perhaps causing this phenomenon. This knowledge gained significance when installing the gages into the scramjet engine in freejet facilities, where access was extremely limited, and monitoring of gage behavior was impossible.

\subsection{RBCC Tests}

The goal of this initial skin friction sensor design was to validate its use in scramjet engine flowfields. An opportunity to do this was found in another NASA project. The object of study in this program was a concept for a single stage to orbit (SSTO) vehicle that uses different thermodynamic cycles during different phases of the flight profile. The engines of this vehicle are based on a concept called the "rocketbased-combined-cycle," or RBCC. The engines of this particular design integrate a 
rocket into an annular duct with a movable inlet spike. At take off, the rocket alone provides thrust. As flight speed increases, the engine becomes a ducted rocket before shutting down the rocket and transitioning to ramjet operation. Once the speed increases to about Mach 5 or 6 , the engine transitions from ramjet to scramjet operation. As the vehicle leaves the atmosphere, the flow through the engine stops and the rocket motor is again employed for the remainder of the flight. The program was intended to design and test such an integrated engine to learn about the possibility of efficiently combining the different engine cycles into a single engine.

Tests of the engines of this concept have been undertaken in several different facilities under several of the different flow regimes. Of interest to the current work were tests of the engine operating in the scramjet mode. These tests provided an opportunity to test the skin friction sensor in a scramjet engine environment similar to the environment expected for the Hyper-X.

\subsubsection{Test Facility}

Tests of the RBCC engine operating in the scramjet mode were conducted at the General Applied Sciences Laboratory (GASL) in Ronkonkoma, New York. GASL operates several different wind tunnels for high-speed aerodynamics and hypersonic air breathing propulsion tests (see Figure 30). The facility used for these tests was the Leg IV freejet facility, which is described in detail below.

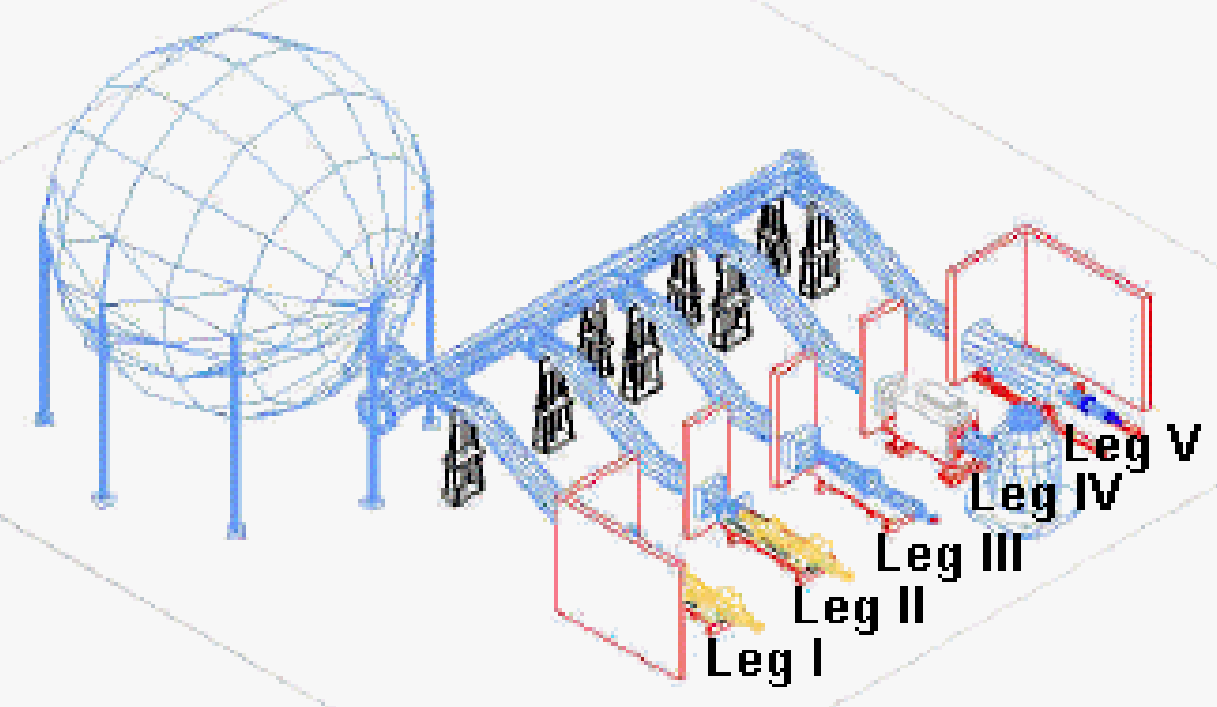

Figure 30: GASL's Blowdown Test Facilities ${ }^{40,41}$ 
The Leg IV facility can produce test gas at temperatures up to $5000^{\circ} \mathrm{R}$. This is accomplished by using a ceramic bed storage heater to first heat the air before diluting with cold air. A combustion booster section (vitiator) that follows can be used to achieve the higher temperatures. For cases where the test gas temperature is between $800^{\circ} \mathrm{R}$ and $2500^{\circ} \mathrm{R}$, the pebble bed alone is used and an automated system controls the cold air dilution to achieve the desired temperature. When temperatures between $2500^{\circ} \mathrm{R}$ and $5000^{\circ} \mathrm{R}$ are required, hydrogen is added in the vitiator and is burned. Oxygen is also added with the hydrogen in order to replenish the oxygen consumed by the combustion process. The hydrogen to oxygen ratio is controlled such that a concentration of oxygen equal to that of stoichiometric air is maintained. Figure 31 shows the storage heater and the oxygen and hydrogen injection section. The storage heater is the large gray object in the center, and the booster system consists of the hydrogen and oxygen supply lines connected to the nozzle ${ }^{41}$. After heat addition, the flow enters the nozzle, where it is expanded to the Mach number associated with that nozzle. The nozzle exits into the test cabin, where the freejet flows in and around the test article. At the aft end of the test cabin, the flow enters a diffuser, which then leads into a large vacuum sphere.

Figure 32 gives a view of the test cabin interior with a scramjet model from previous tests installed. Figure 33 shows the operating envelope of the Leg IV facility.

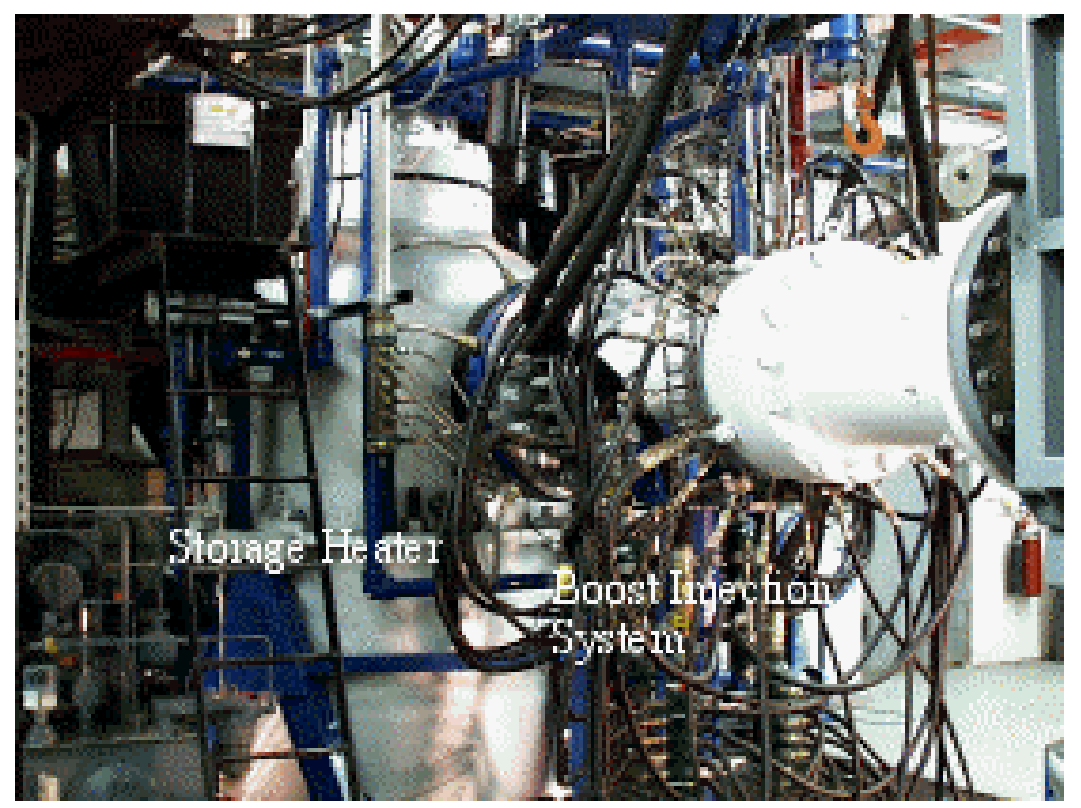

Figure 31: GASL Leg IV Heating Systems ${ }^{41}$ 


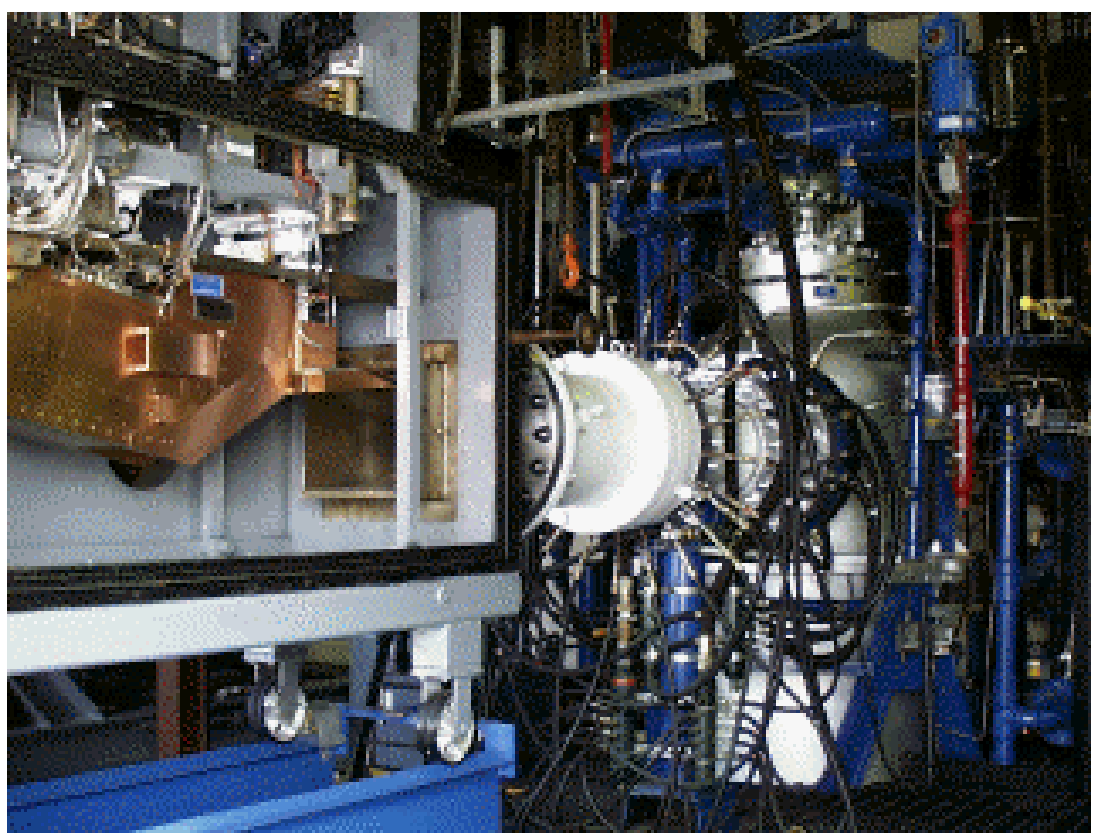

Figure 32: GASL Leg IV Test Section (not the same as used in RBCC tests) ${ }^{41}$

For aerodynamic testing where high enthalpies are not required, Mach numbers up to 12 can be reached with the maximum pressure and the preheater alone. Mach numbers from 2 to 8 with true flight enthalpy can be produced with the booster heater ${ }^{41}$.

For the RBCC tests, this facility provided a nominal Mach 6.4 freestream with

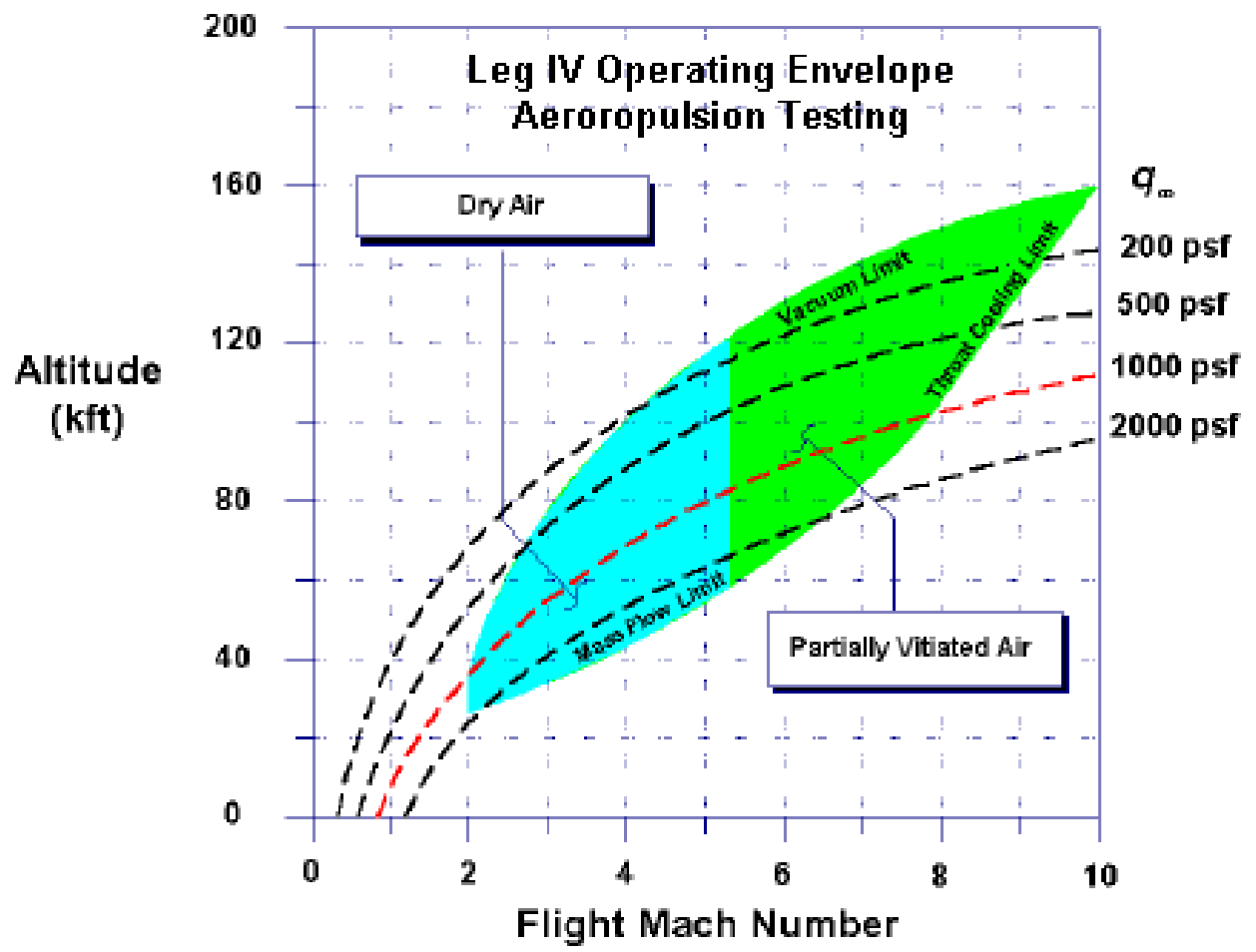

Figure 33: GASL Leg IV Operating Envelope ${ }^{41}$ 
total pressure and temperatures of $\mathrm{P}_{0}=1350 \mathrm{psia}(9310 \mathrm{kPa})$ and $\mathrm{T}_{0}=2800{ }^{\circ} \mathrm{R}(1555$ $\left.{ }^{\circ} \mathrm{K}\right)$. To reach the desired total enthalpy (and hence total temperature) of these tests the air was preheated by the pebble bed and further heated by vitiation.

One skin friction gage (Gage \#1) was mounted into an oblong copper plug, which was located along the centerline of the engine, downstream of several fuel injectors. The engine model was a roughly $210^{\circ}$ annulus, with the flow path following a varying radius. A sketch of the engine model and the skin friction sensor location is shown in Figure 34.

Since the facility was a freejet, the engine model was completely housed within the test cabin. All electrical and mechanical connections to the engine were therefore carefully routed from the engine to the test cabin walls, where they were passed through sealed junctions. The space around the engine inside the test cabin was understandably tightly packed with all the necessary instrumentation and plumbing. This made installation of the skin friction sensor challenging. This was due to the fact that not only electrical connections were required, but also multiple water lines had to be routed from a supply manifold to the test cabin pass-through, and then inside the test cabin to the skin friction sensor and then back out of the test cabin and outside the building, where the water was dumped. Despite these difficulties, the installation was accomplished with the aid of GASL's skilled employees.

The cooling water was connected to both the internal and external lines from a manifold at $80 \mathrm{psi}(552 \mathrm{kPa})$. A 2310 signal-conditioning amplifier provided 5 Volts of excitation to the Wheatstone bridge of the skin friction gage, as well as an amplification of 1000. Data was recorded on the facility data acquisition system at $20 \mathrm{~Hz}$.

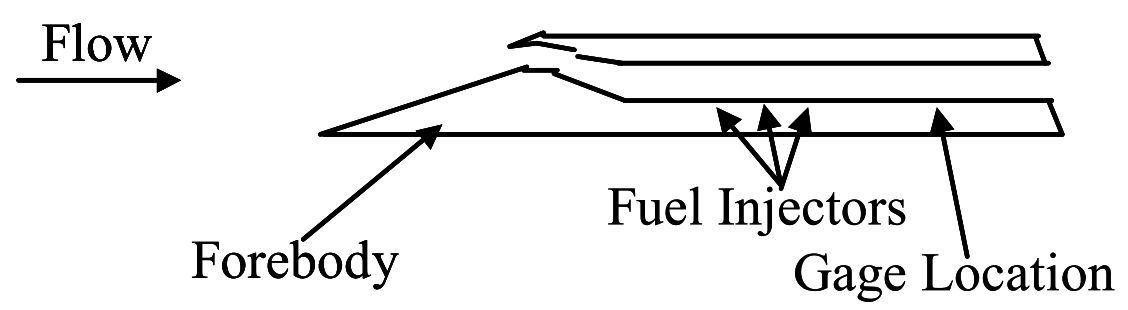

Figure 34: RBCC Engine Skin Friction Sensor Location 


\subsubsection{Test Results}

A series of tests of the RBCC engine were conducted in GASL's Leg IV facility with the skin friction sensor installed. Typical run times were about 30 seconds, with fuelling changes occurring periodically throughout the test cycle. Data was collected for roughly 120 seconds, recording both before and after the tunnel was operating. During the first few seconds of tunnel operation, the forebody cone of the engine was traversed in order to swallow the shock and start the inlet. This was followed by several seconds of steady, un-fueled flow, after which fuel was injected from different locations in sequenced stages. During testing nearby wall temperatures, measured by thermocouples near the gage, reached 850 to $900{ }^{\circ} \mathrm{R}\left(470\right.$ to $\left.500{ }^{\circ} \mathrm{K}\right)$.

Only one axis of the skin friction sensor was available for measurement during these tests due to previous damage to the gage. The gage was positioned in the engine such that the axial flow was aligned with the sensing axis of the skin friction sensor. Since the gage was located along the centerline of the model, the flow should have been symmetric and nearly two-dimensional.

Three successful tests were conducted with the skin friction gage over the course of one week. Since the gage was enclosed in the wind tunnel without access, the gage ceased to function after these tests. This was likely due to the loss of oil and the continued exposure to the high enthalpy flow environment without that protection. The results of these three tests are shown in Figure 35, Figure 36, and Figure 37. Table 3 summarizes the maximum levels of $\tau_{\mathrm{w}}$ and $\mathrm{C}_{\mathrm{f}}$ for each run. As in the supersonic wind tunnel, the results showed good repeatability.

Each run had the same tunnel parameters and operation procedure, but the specific timing and fuel addition parameters were different. At the initial tunnel start up the skin friction coefficient for each run quickly reached a value of $\mathrm{C}_{\mathrm{f}} \approx 0.0027-0.003$. This corresponded to wall shear levels of $\tau_{\mathrm{w}} \approx 485-525 \mathrm{~Pa}$, which was within the expected nominal range of 300-500 $\mathrm{Pa}$. In the following several seconds, the centerbody moved into position and the skin friction level remained fairly constant. The graphs indicate the time at which the centerbody reached the specified position and ceased 


\begin{tabular}{|c|c|c|c|}
\hline Run & $\tau_{\mathrm{w}, \text { start }}(\mathrm{Pa})$ & $\mathrm{q}(\mathrm{Pa})$ & $\mathrm{C}_{\text {f,start }}$ \\
\hline 21 & 485 & 170775 & 0.00284 \\
\hline 22 & 525 & 166600 & 0.00315 \\
\hline 23 & 520 & 188630 & 0.00276 \\
\hline
\end{tabular}

Table 3: RBCC Test Results Summary

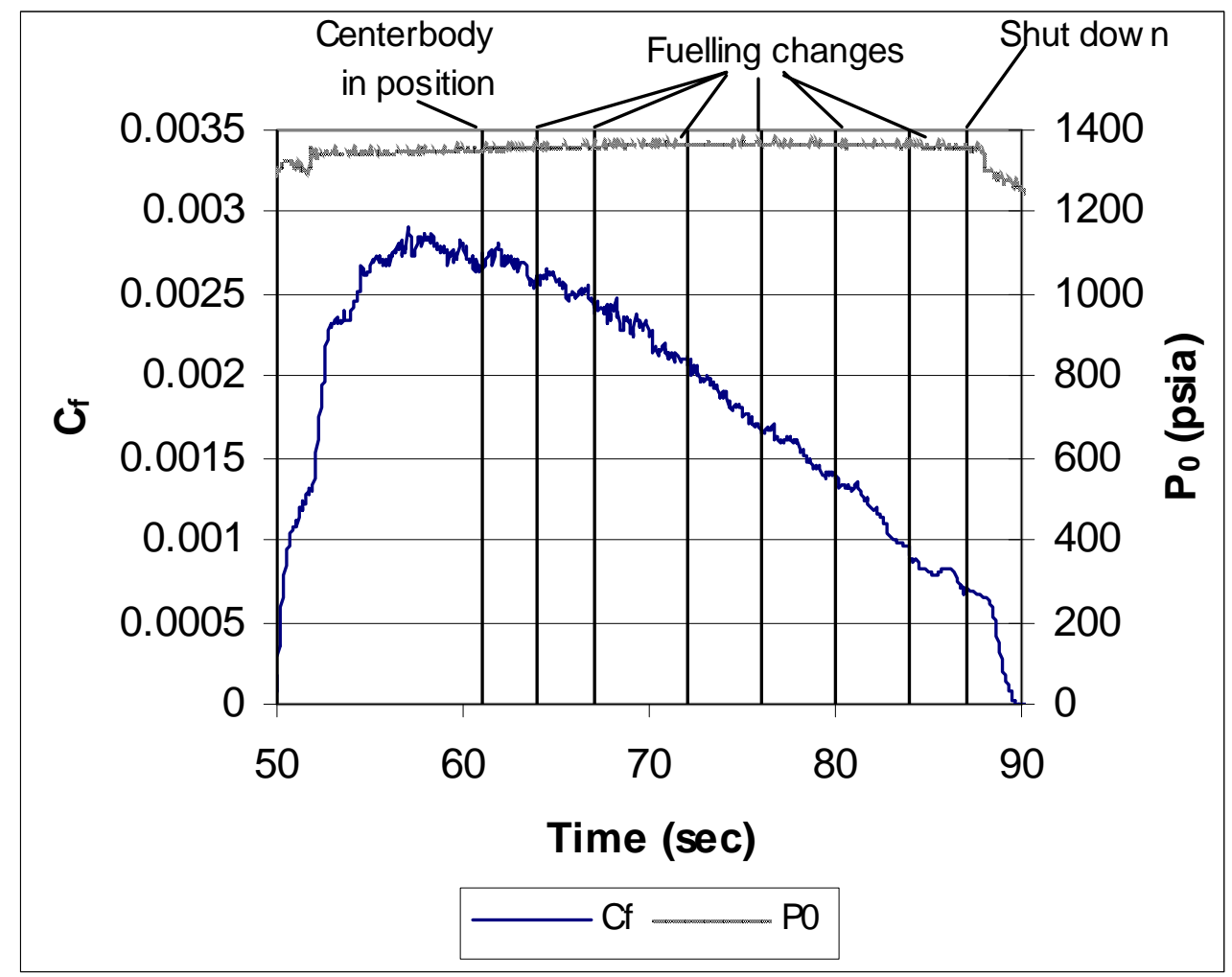

Figure 35: RBCC Test 21

movement. Next, fuel was added, and the graphs also indicate the times at which fueling changes were made. The skin friction output steadily fell to minimum values of $\mathrm{C}_{\mathrm{f}} \approx$ 0.0007-0.0015 during and after fueling changes. After the shut down of the tunnel, the output returned to zero in each run, indicating little or no thermal zero shifts. 


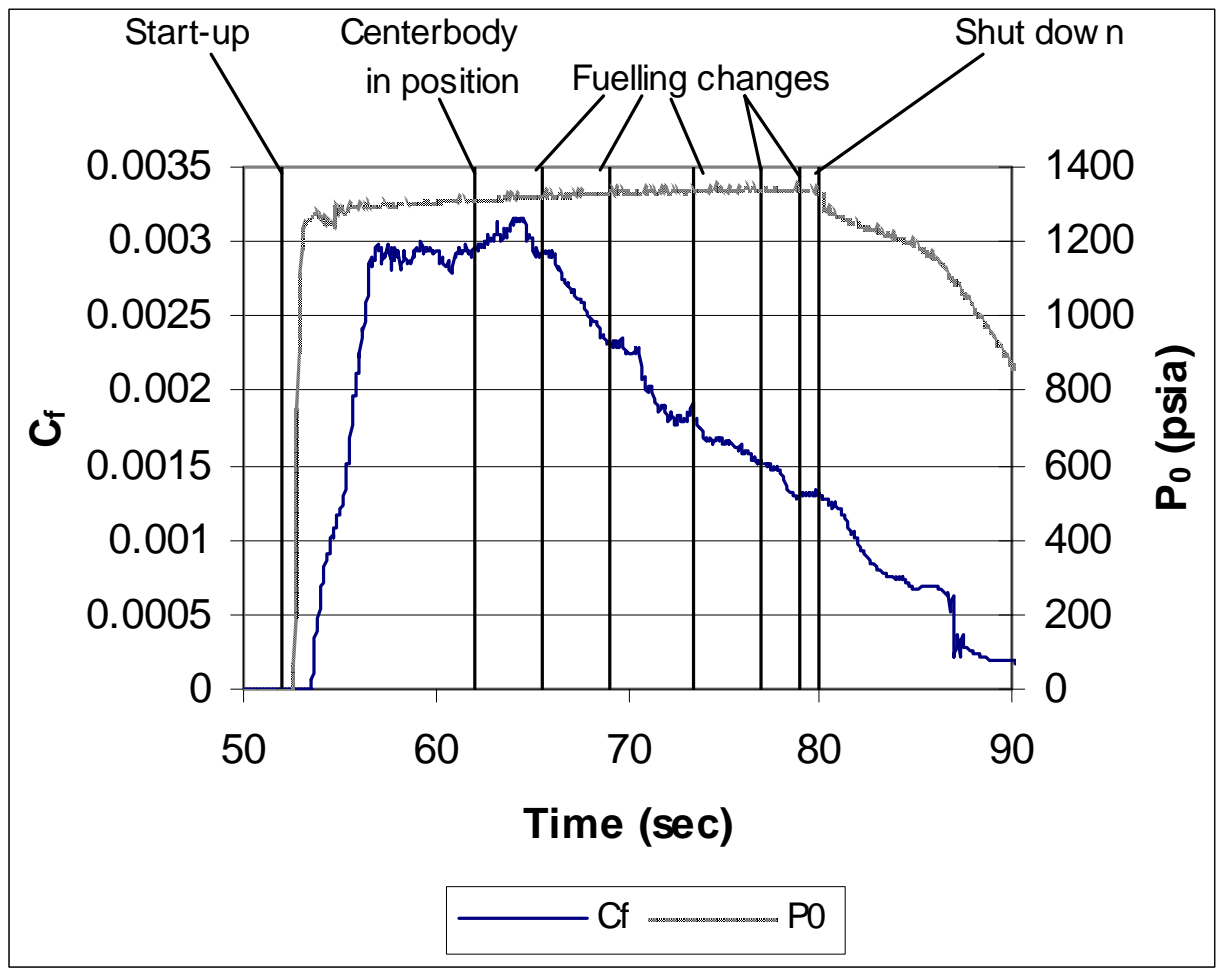

Figure 36: RBCC Test 22

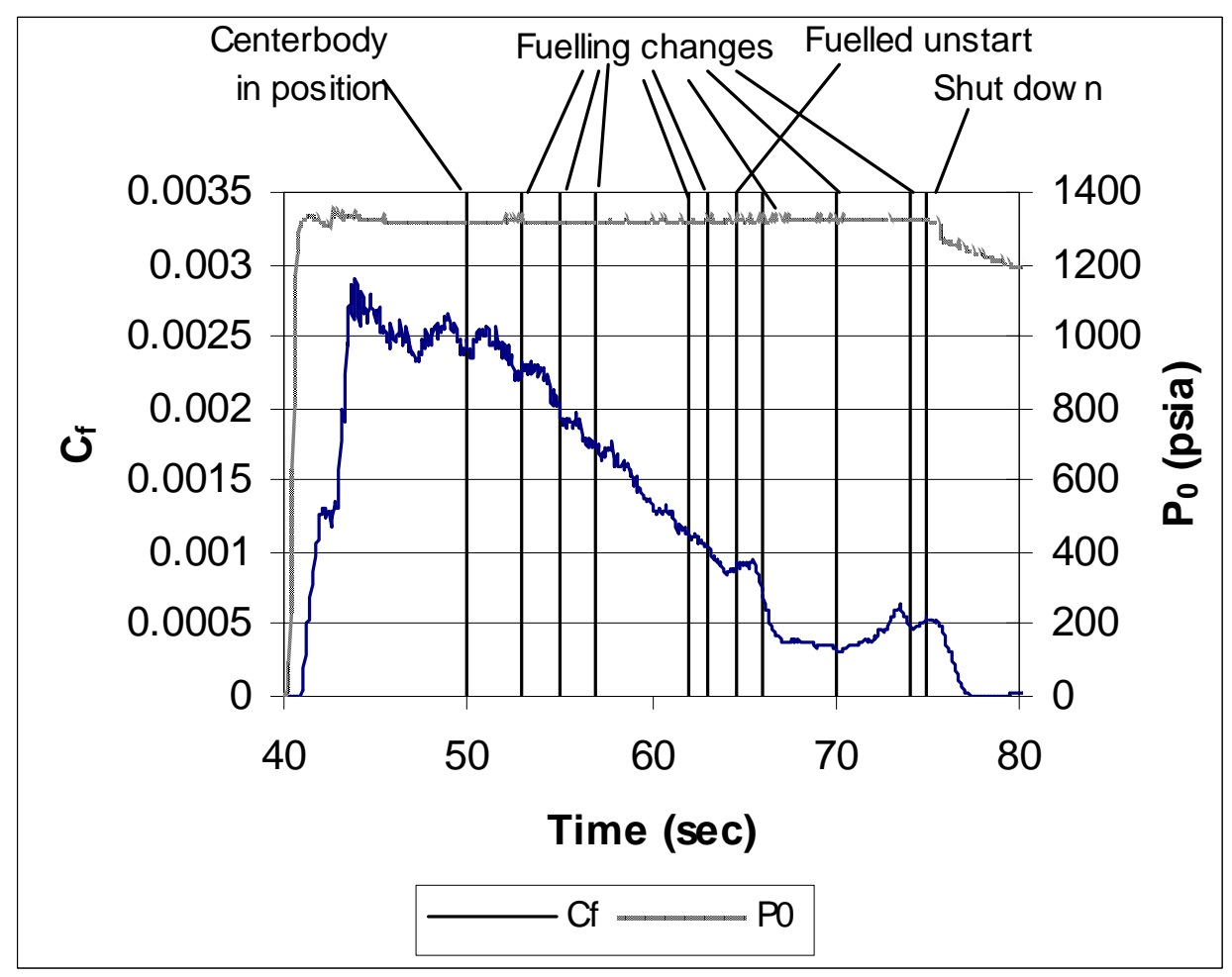

Figure 37: RBCC Test 23 
In Run 22 and Run 23 (Figure 36 and Figure 37), the steady portions of the signal are clearer. There are clear steady portions at the beginning of these runs, and steady portions can even be seen between fueling changes in some instances. However, there appear to be time lags between the nominal change in fueling scheme and the change in output.

Run 23 also included an especially interesting event. About 64 seconds into the run, enough fuel was added in the combustor to unstart the inlet (see the time marker in Figure 37). Subsequently, the flow in the engine went from supersonic to subsonic. This event can clearly be seen in the output of the skin friction sensor. After a delay of a few seconds, the output dramatically dropped in response to the significantly reduced flow rate. The signal remained relatively constant for the last few seconds, rising slightly as fuel continued to be added, before returning to zero as the tunnel was shut down.

When calculating the skin friction coefficient, it is important to consider the dynamic pressure used to normalize the wall shear. The definition of the skin friction coefficient specifies that the local wall shear should be non-dimensionalized by the local dynamic pressure. For $q=1 / 2 \rho V^{2}$, the density, $\rho$, and the velocity, $V$, should be taken at the edge of the boundary layer at the same position as the wall shear. Likewise, if the dynamic pressure is calculated from $\mathrm{q}=1 / 2 \gamma \mathrm{PM}^{2}$, then the ratio of specific heats, $\gamma$, the pressure, $\mathrm{P}$, and the Mach number, $\mathrm{M}$, should also be taken at the local boundary layer edge. However, for the RBCC tests the dynamic pressure inside the combustor was unknown due to a lack of necessary instrumentation and prediction tools for the harsh environment. The standard practice when this type of situation occurs is to normalize the wall shear with the freestream dynamic pressure ${ }^{35}$. The freestream pressure was obtained from static pressure measurements taken at the exit of the facility nozzle. The Mach number and ratio of specific heats were later calculated by GASL based on the temperature and gas composition measured at the nozzle exit. This information provided a value for the freestream dynamic pressure that remained nearly constant throughout each run.

The dynamic pressure inside the combustor does not remain constant during each run, however. The inlet shocks reduce the total pressure, and the dynamic pressure also. When heat is added as the fuel is injected and burned, the total and dynamic pressures 
decrease further. Therefore, the dynamic pressure in the combustor would likely decrease to significantly lower than the freestream dynamic pressure. A factor of $1 / 6$ is a very rough estimate of the ratio of combustor dynamic pressure to freestream dynamic pressure. And there could have been separation, wall cooling by unburned fuel, or some other unexpected behavior of the boundary layer at the measurement location. Keeping all of this in mind, it becomes apparent that a $\mathrm{C}_{\mathrm{f}}$ calculated based on local dynamic pressure could have a different value than one based on freestream dynamic pressure.

The results from these tests in the scramjet combustor of the RBCC engine were considered an indication of success. The skin friction sensor survived the scramjet combustor environment and provided results that were repeatable and believable. Both the wall shear and skin friction coefficient were close to the values expected from experience and estimates provided NASA. The cooling system performed the required task of preventing thermal effects from contaminating the signal, which was no small accomplishment since the local wall temperatures reached $900^{\circ} \mathrm{R}$. The objective of designing a skin friction sensor for the Hyper-X flight vehicle engine seemed to be well on its way. 


\section{CHAPTER FIVE:}

\section{GAGE DESIGN TWO- HXEM TEST DESIGN}

\subsection{Gage Design}

Once the design of the first skin friction sensor had been proven by the successful tests in the RBCC scramjet engine, the plan was to evolve that skin friction sensor design for wind tunnel tests of a model of the Hyper-X engine. Moving from the first wind tunnel scramjet flow environment to a wind tunnel test of the Hyper-X Engine Model (HXEM) gave an incremental approach to meeting the demands of the Hyper-X flight vehicle engine. Therefore, the design of the second skin friction sensor followed almost exactly that of the first, with what were thought to be some minor improvements.

\subsubsection{Gage Description}

The second skin friction gage design is shown in schematic form in Figure 38. As can be seen by referring to Figure 18, the geometry of the floating head and beam are unchanged. The base, however, was redesigned, as was the internal cooling system. The base was redesigned to reduce the overall diameter of the sensor and to increase the amount of flow to the internal cooling lines. The internal cooling system was also redesigned to make use of larger tubes, since the hypodermic tubing used in the first skin friction sensor were somewhat fragile.

As in the first design, four semiconductor strain gages were bonded to the base of the beam, one on each of four orthogonal flats, and they were wired into two halfWheatstone bridges, one each for the streamwise and cross-stream axes of flow. The other details concerning the floating element and the beam are the same as were discussed in Section 4.1.1. The specific details of the redesigned components are described below.

The base was again made of brass. The outer diameter of the base was 0.500 inch $(1.27 \mathrm{~cm})$, and the main portion of the base was 0.500 inch $(1.27 \mathrm{~cm})$ tall. However, a protrusion of the base with outer diameter of 0.250 inch $(0.635 \mathrm{~cm})$ was extended a distance of 0.375 inch $(0.953 \mathrm{~cm})$ below the main portion of the base. A blind hole was 


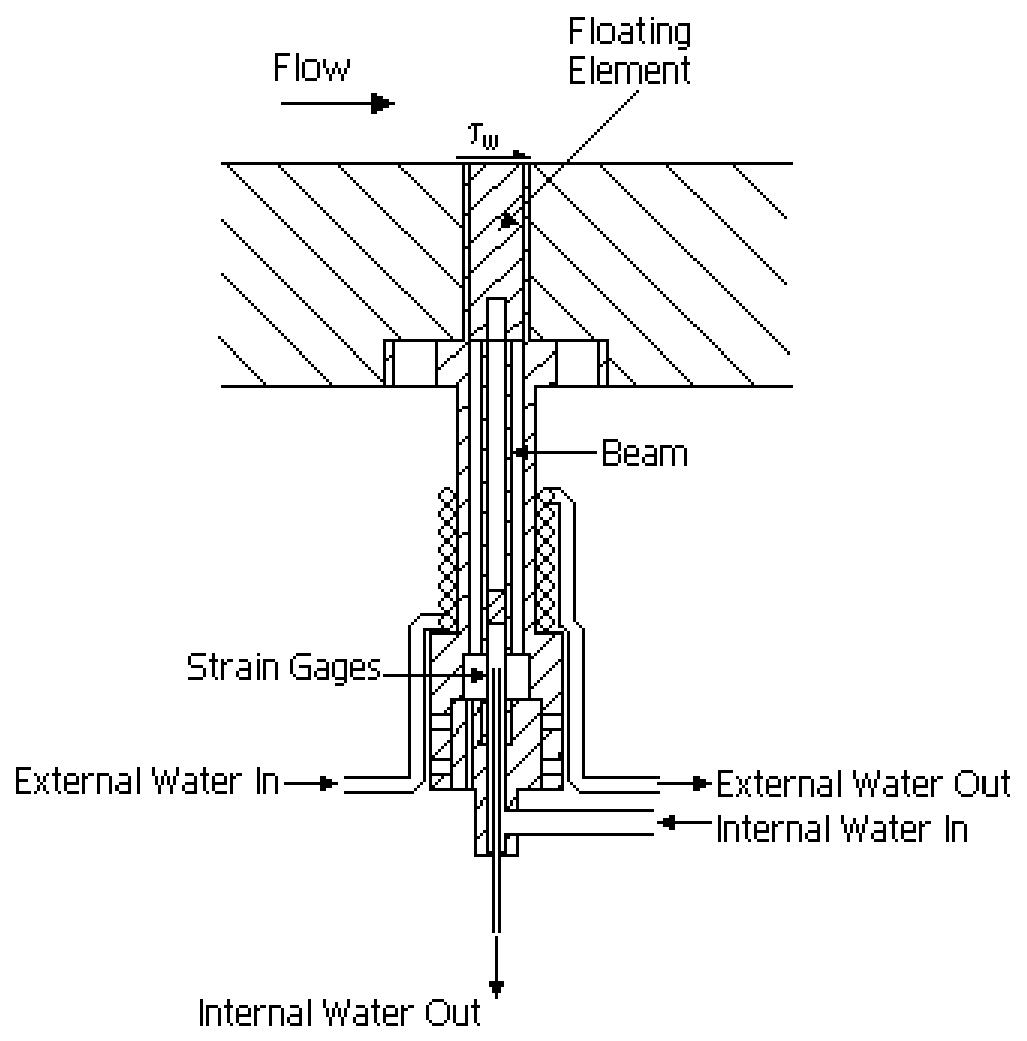

Figure 38: Gage Design Two Sketch

drilled in the center 0.250 inch $(0.635 \mathrm{~cm})$ deep for the beam to be mounted into. A smaller blind hole was drilled in the center almost completely through the base, leaving just 0.020 inch $(0.0508 \mathrm{~cm})$ from the bottom of the extension. Within this hole, a through-hole of diameter 0.0625 inch $(0.159 \mathrm{~cm})$ was then drilled completely through the center of the base. Two through-holes of 0.052 -inch $(0.132 \mathrm{~cm})$ diameter were drilled off center for the wire leads from the strain gages. Another through-hole of 0.020 -inch $(0.0508 \mathrm{~cm})$ diameter was drilled to allow a thermocouple to be inserted near the strain gages. Lastly, a hole was drilled into the side of the extension of the base with a diameter of 0.125 inch $(0.318 \mathrm{~cm})$.

A 0.0625 -inch $(0.159 \mathrm{~cm})$ diameter tube was inserted through the center throughhole of the base to a distance near the location of the strain gages. A 0.125 -inch $(0.318$ $\mathrm{cm})$ tube was then inserted into the hole in the side of the base's extension. Both tubes were soldered into place. This arrangement formed the internal cooling system. Water was supplied to the 0.125 inch tube and flowed into the extension of the base, around the 0.0625 inch tube, and up through the base and into the beam. A setscrew threaded into 


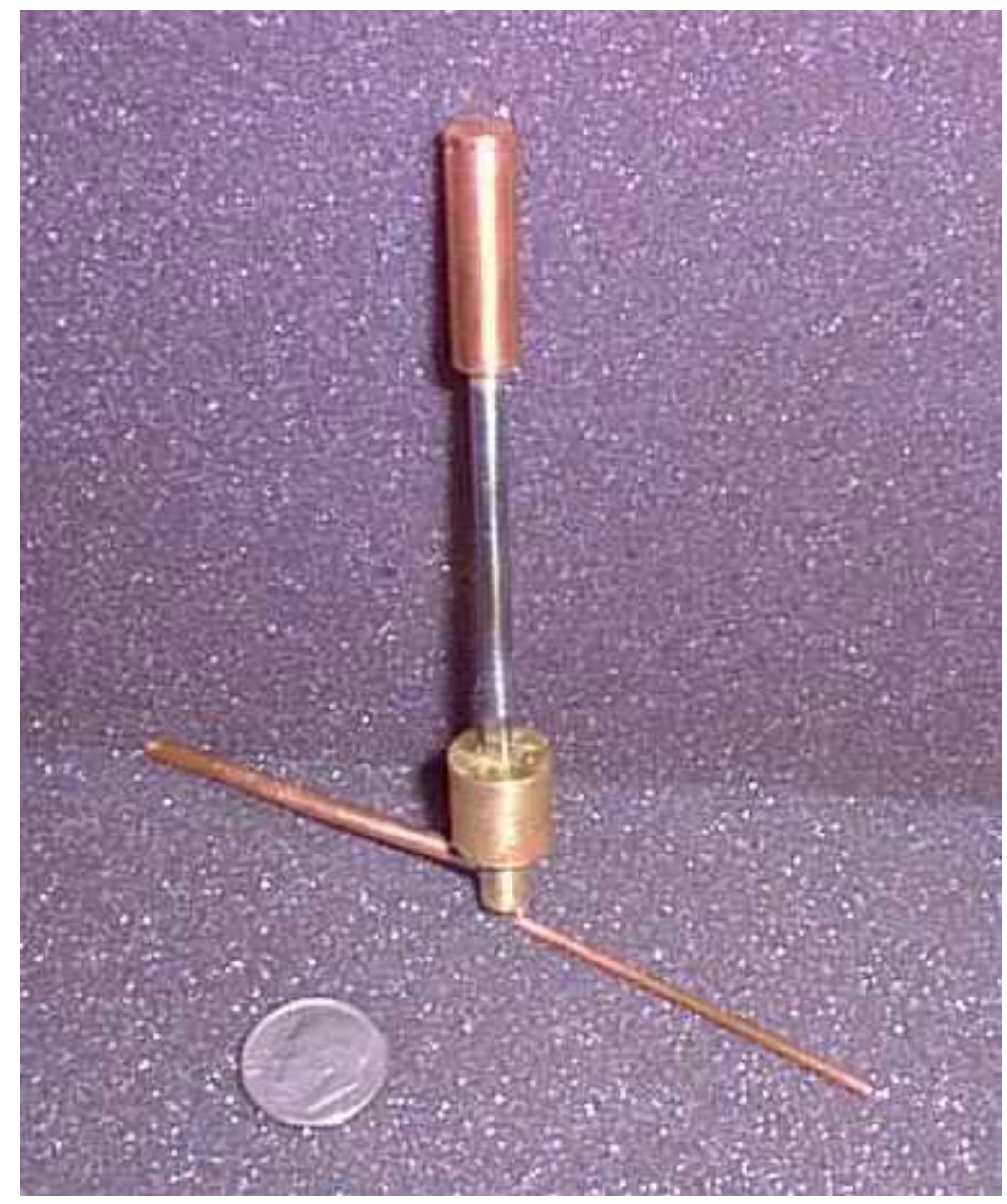

Figure 39: Gage Design Two Internal Components

the beam just above the height of the strain gages, forced the flow to enter into the end of the 0.0625 -inch tube, and exit the skin friction sensor. This system required fewer tubes than the first skin friction sensor. By using larger tubes the amount of cooling flow into the gage and the ruggedness of the gage were improved. A photo showing the internal components (floating head, beam, base, and internal cooling tubes) is shown if Figure 39.

The housing geometry was similar to the first design, with the same sleeve and mounting flange at the top. The lower portion of the housing was somewhat different from the first design. The diameter at the bottom of the housing was smaller, since the base that fit inside the housing was smaller. Also, the base was mounted in the housing using eight setscrews. Using the setscrews allowed the position of the head-beam-base assembly to be adjusted. This feature made it easier to ensure that the floating head was level with the top surface of the housing, and that the head was centered within the housing. Instead of using an O-ring to seal the base in the housing, two-part epoxy was 


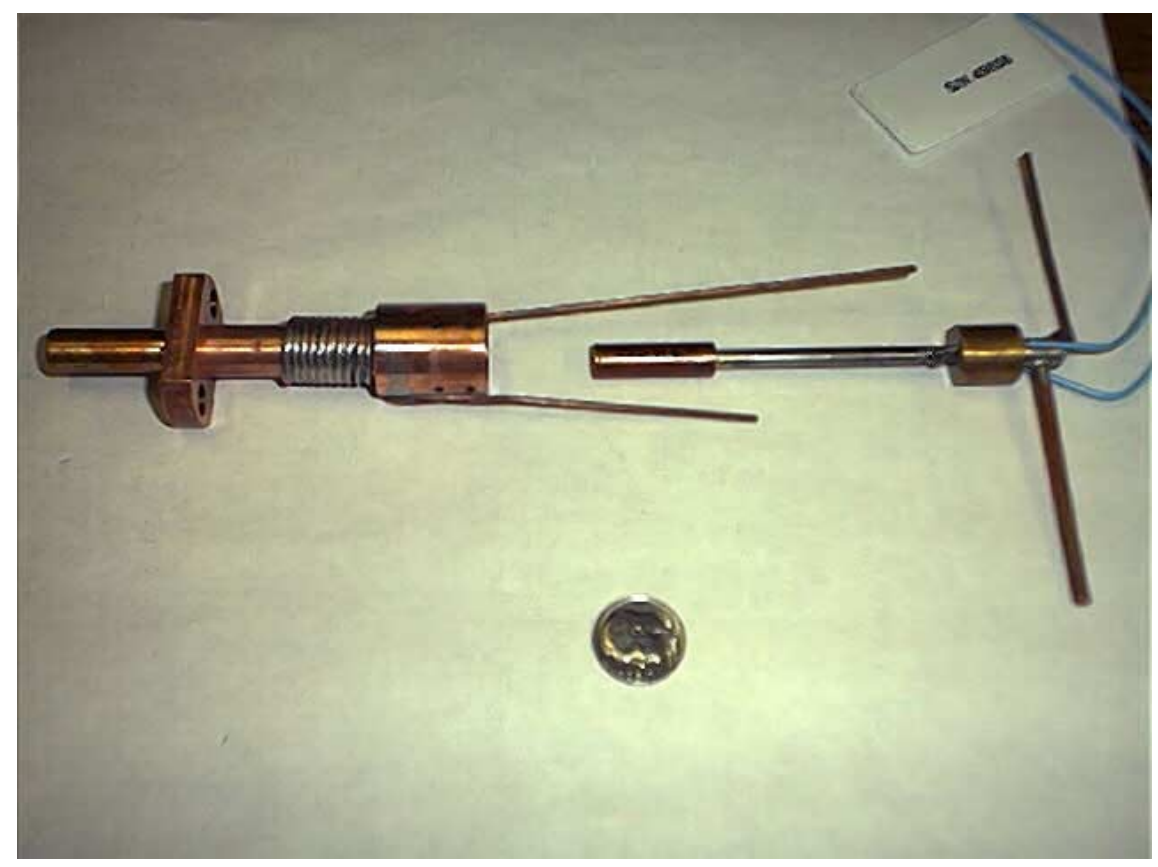

Figure 40: Design Two Housing and Internal Components

used on all seams around the base of the housing. Once again, 0.0625 inch $(0.159 \mathrm{~cm})$ tubing was coiled around the outside of the housing to provide external cooling lines. Five sensors of this design were built. Figure 40 shows a floating head-beam-base assembly ready to be mounted into a housing. Three completed and assembled gages with heat resistant wires and connectors are shown in Figure 41.

No further structural analysis was done on this design since the beam and floating

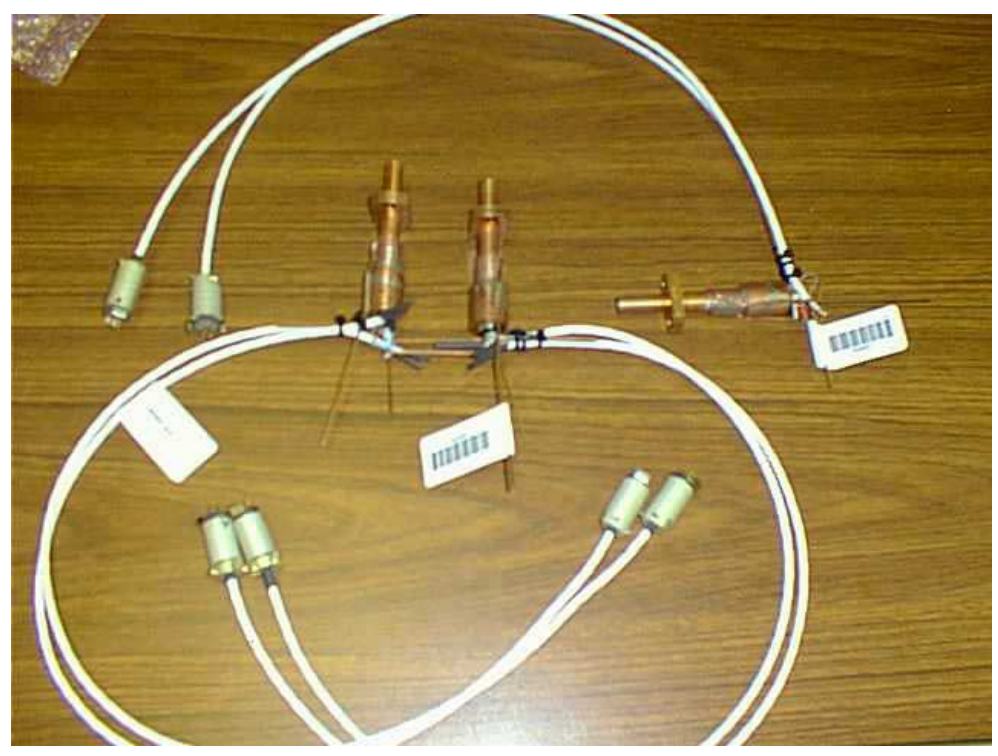

Figure 41: Three Assembled Gages (Design Two) 
element were the same as were used in the first design. The design shear loads were the same, and since the first gage had worked well under that loading, no changes were needed to increase sensitivity or reduce deflections.

\subsubsection{Oil-Fill Special Concerns}

There was one significant difference in the testing configuration that did raise concern about this design. The engine of the Hyper- $X$ is located on the underside of the vehicle, and it is roughly like a box-shaped duct formed by a cowl. Since the cowl is not very thick, the skin friction sensors were required to be mounted on the body side, which meant that they would be upside down. This was not an issue in the RBCC tests since the gage was oriented right side up in that engine. But, oil leakage now became a major concern for these HXEM tests.

To address this issue, higher viscosity oil was used to fill the interior space of the skin friction sensor. It was believed that the higher viscosity oil would leak much more slowly from the gap around the head when the gage was inverted. In the first design, 1000 centistokes (cSt) silicon oil had been used. But for this design 10,000 cSt oil was used.

However, the higher viscosity oil proved to be even more difficult to get into the gage. The same method of using a vacuum to remove air bubbles from within the housing was used. But, to escape from the gage interior the bubbles now were required to travel through the higher viscosity oil and still fit through the same 0.005 -inch gap around the head. While in the vacuum chamber, bubbles were observed to reach the surface of the oil reservoir at a much slower rate than with the lower viscosity oil. And, though the time between bubbles might become quite long- several minutes- it seemed that there were always more. Thus, it was difficult to know whether all of the air had ever been finally removed from the interior of the skin friction gages.

Again, any air bubbles that remained within the housing would grow in the very low pressure of the test cabin. As they grow, they could exert a force on the beam and/or force more oil out of the sensor. It was also unknown what effect rapid leaking of oil would have on the skin friction measurement if it occurred during testing. 


\subsection{Calibration Results}

The Design Two skin friction gages were calibrated with the static calibration method as described earlier. These gages were also calibrated in the glycerin flow of the dynamic calibration rig.

A sample of the static calibration results for one of these gages is shown in Figure 42. Good linearity was again seen in the results of the linear fits to the calibration data. The sensitivity of these sensors was close to, but slightly less than that of the first design. Slight cross-talk was also seen, similar to the first design. All five sensors were calibrated along each axis, in both the positive and negative directions.

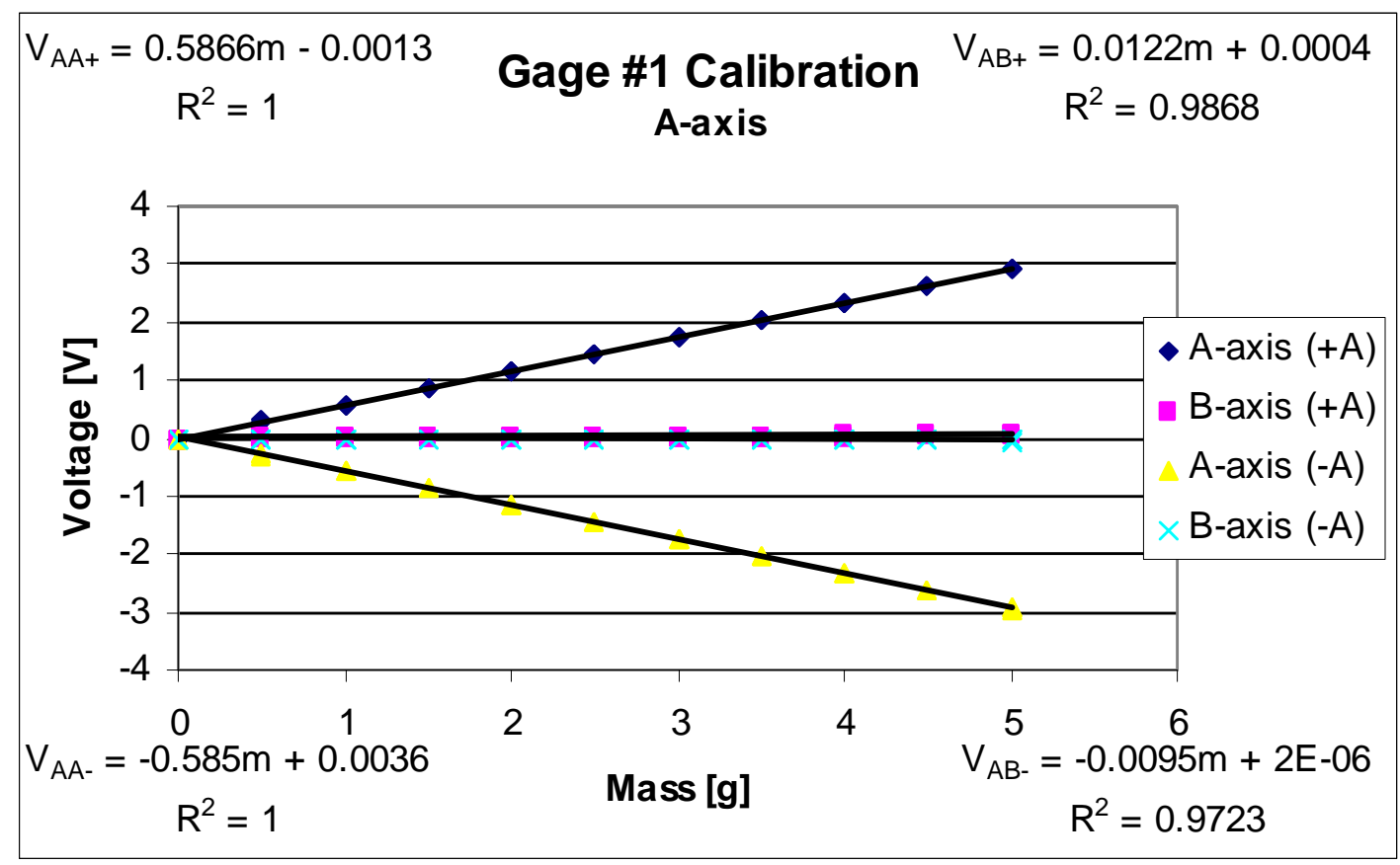

Figure 42: Sample Static Calibration for Design Two

The above results were obtained without the cooling water running in the internal cooling lines. When these sensors were calibrated with cooling water flowing in the internal cooling lines, unexpected output was observed. Fluctuations of roughly 0.040 volts, with occasional excursions up to 0.150 volts, were seen when the flow was running through the internal cooling lines. The fluctuations were also not the same for the two axes of the same gage, and would change with the orientation of the gage. For instance, the fluctuations for one gage were larger when the gage was vertical, but were slightly less when horizontal. It was found to be necessary to use a pressure control valve on the internal cooling lines. By lowering the flow rate into the internal cooling lines, the 
magnitude of the fluctuations was minimized. This experience, though not understood at the time, was the first indication of a serious flaw in the design of the internal cooling system.

The calibration rig was also used to calibrate these sensors. It was known that when the storage tank of the calibration rig was filled to the top, the wall shear inferred from the measured pressure gradient within the channel was $104 \mathrm{~Pa}$. This provided a single calibration point to compare the output of the static calibration to that of the dynamic calibration. By filling to different heights within the storage tank, different values of wall shear can be generated. But, in order to know what the shear level is in these cases, the pressure transducers in the channel must be used to measure the pressure drop along the channel. These pressure transducers, however, were not operational at the time of the calibration of these gages. Therefore, only the single reference point of 104 Pa was used.

Figure 43 shows a sample result of the dynamic calibration of a Design Two skin friction sensor. Figure 43a shows the raw voltage output over time. The output started at zero, and jumped to just over 0.3 Volts as the channel valve was opened. The output remained very constant during the steady portion of the run and returned to zero when the valve was closed again. The raw voltage was multiplied by the calibration constant obtained from the static calibration to convert the output to a shear level. This graph is shown in Figure 43b. The average value of the indicated shear level was 104.4 Pa. This is almost exactly the $104 \mathrm{~Pa}$ shear level that the calibration rig was calculated to give. Other calibration rig tests gave similar results, with measured values of shear ranging from about $95 \mathrm{~Pa}$ to about $115 \mathrm{~Pa}$, giving an uncertainty range of about $\pm 10 \%$. Some of these differences may be attributed to uncertainty in the glycerin level in the storage tank. Obtaining good results such as this from the dynamic calibration added confidence to the results of the static calibration method and also proved the effectiveness of the dynamic calibration rig. 


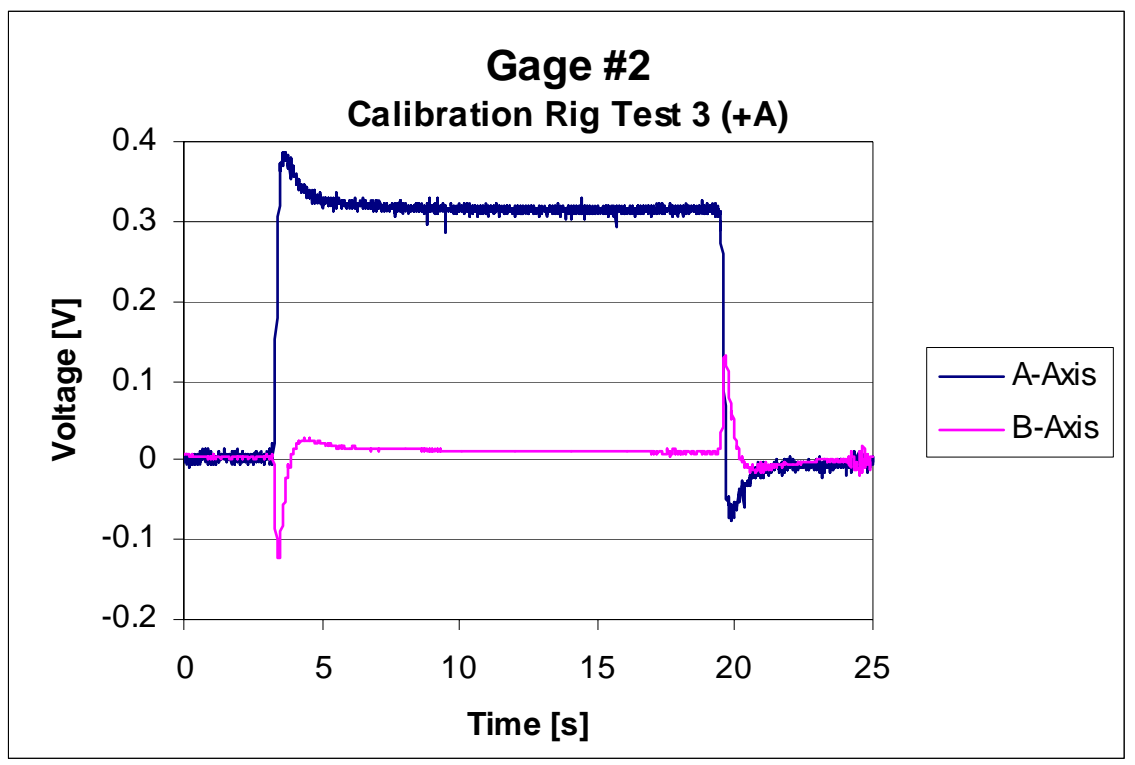

a)

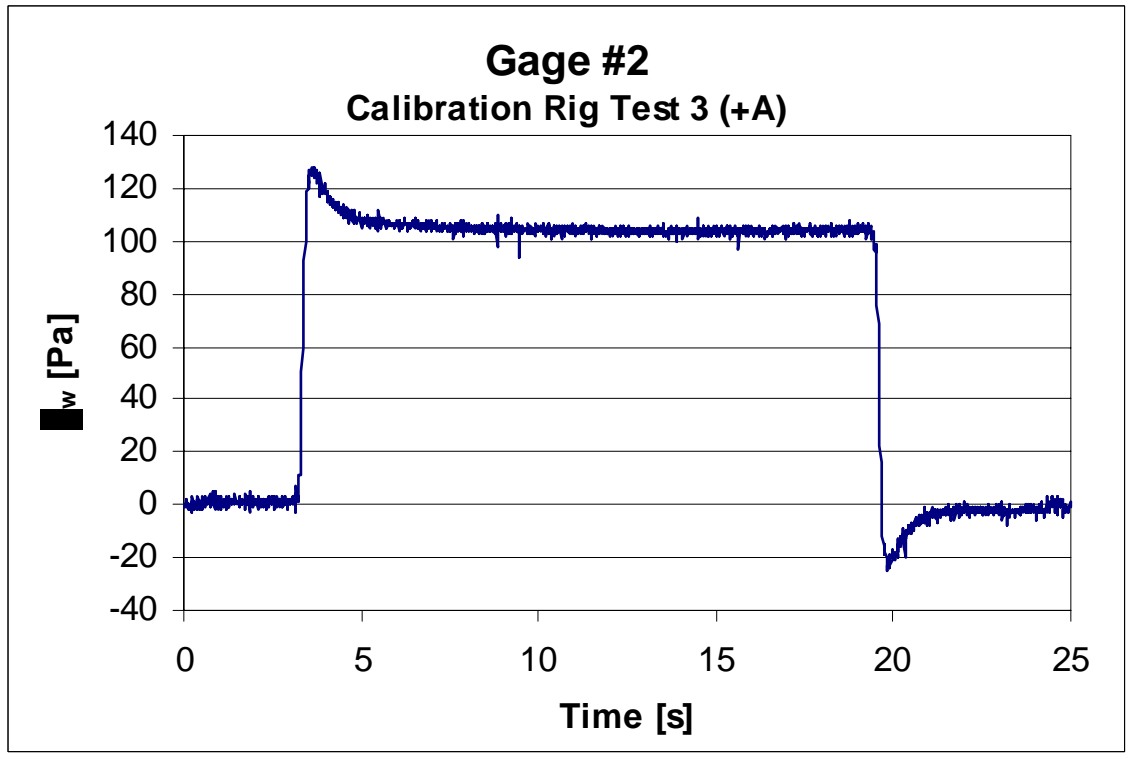

b)

Figure 43: Dynamic Calibration Result- a) Raw Voltage b) Shear Level 


\subsection{Vibration Tests}

Vibration tests were not conducted with the Design Two skin friction sensors. The geometry of the floating element and beam were the same as the first design, so the natural frequency and dynamics of that structure were assumed to also be the same.

\subsection{Cold Flow Tests}

Like all the other skin friction sensors of this study, these gages were tested in the Virginia Tech 9-inch by 9-inch supersonic wind tunnel. Again, the tunnel was operated at a Mach number of 2.4, with total pressure $\mathrm{P}_{0}=50$ psia $(345 \mathrm{kPa})$ and total temperature $\mathrm{T}_{0}=540^{\circ} \mathrm{R}(300 \mathrm{~K})$. The gage was mounted in the same mounting hole in the aluminum floor plate of the wind tunnel. Cooling water was not run in either the internal or external cooling lines during these tests. Data was again sampled at $100 \mathrm{~Hz}$ using the tunnel control and data acquisition software on a PC. The 2310 signal conditioning amplifiers were used to provide a 5-volt excitation, an amplification of 1000, and signal filtering at $10 \mathrm{~Hz}$. The adjacent static pressure port was used in calculating the local dynamic pressure, which was then used to normalize the shear level obtained from the skin friction sensor.

Like the calibration with internal water flow, the results of these tests were not as expected, and were not as good as with the previous skin friction sensor design. Figure 44 and Figure 45 show sample results of two separate runs. The sensor from which these results were obtained was the same sensor from which the dynamic calibration results were obtained. On these graphs, the output from the two axes are labeled as the streamwise $\left(\mathrm{C}_{\mathrm{fx}}\right)$ and cross-stream $\left(\mathrm{C}_{\mathrm{fy}}\right)$ components. The total pressure $\left(\mathrm{P}_{0}\right)$ and static pressure $\left(\mathrm{P}_{\text {inf }}\right)$ traces are also shown.

The shape of the $\mathrm{C}_{\mathrm{f}}$ curve of Figure 44 appeared to be correct, but the magnitude of $\mathrm{C}_{\mathrm{f}}$ was high. The output began and ended at zero, and reached a steady value of about 0.005 during tunnel operation. The expected level of skin friction coefficient, however, was about 0.002 . The run shown in Figure 45 was a little better, yet still left some questions about the accuracy of the skin friction sensor. The output again began and ended at zero. During the steady portion of the run, however, the output slowly 


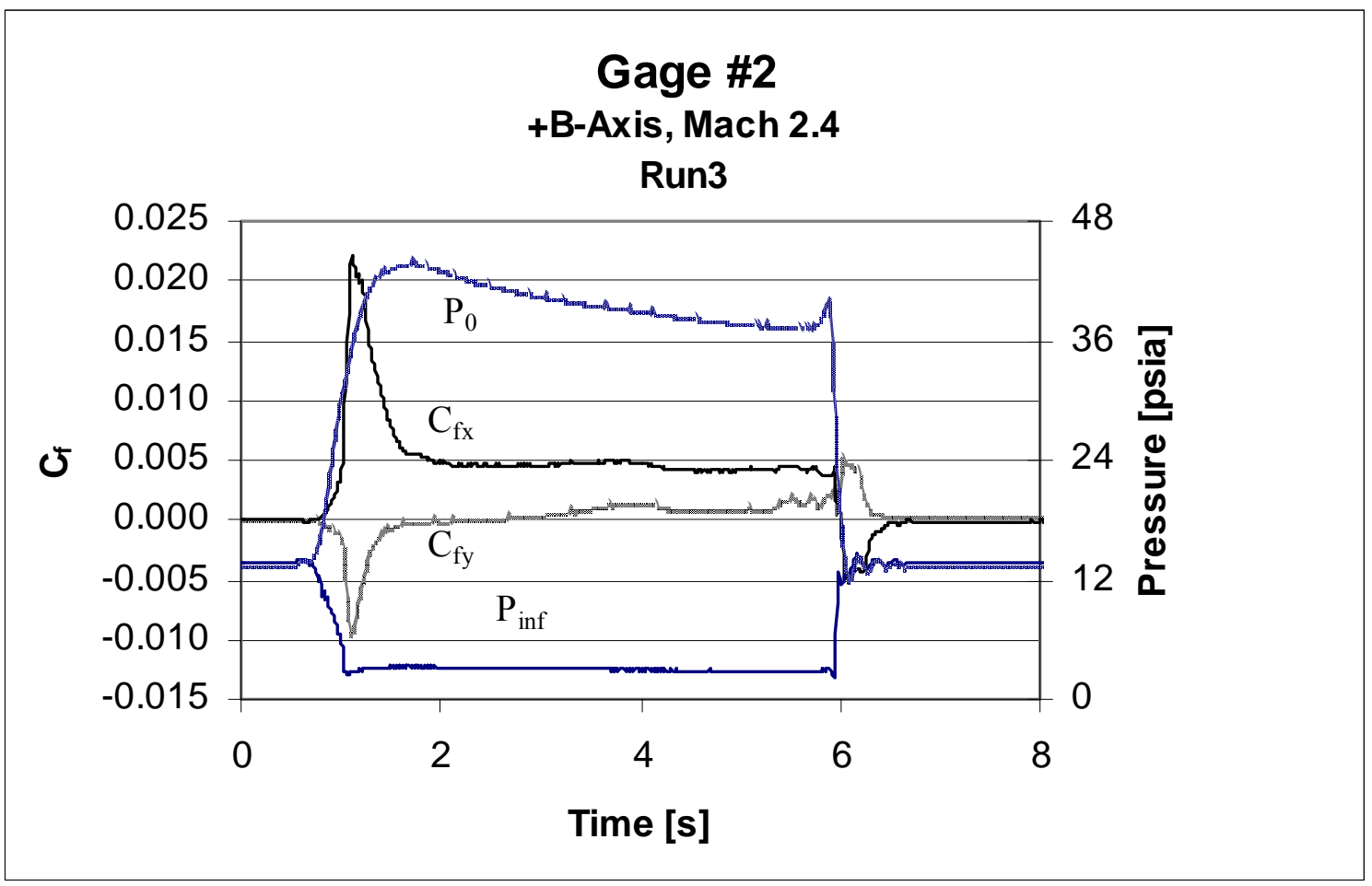

Figure 44: Supersonic Wind Tunnel Test Results: Run 3

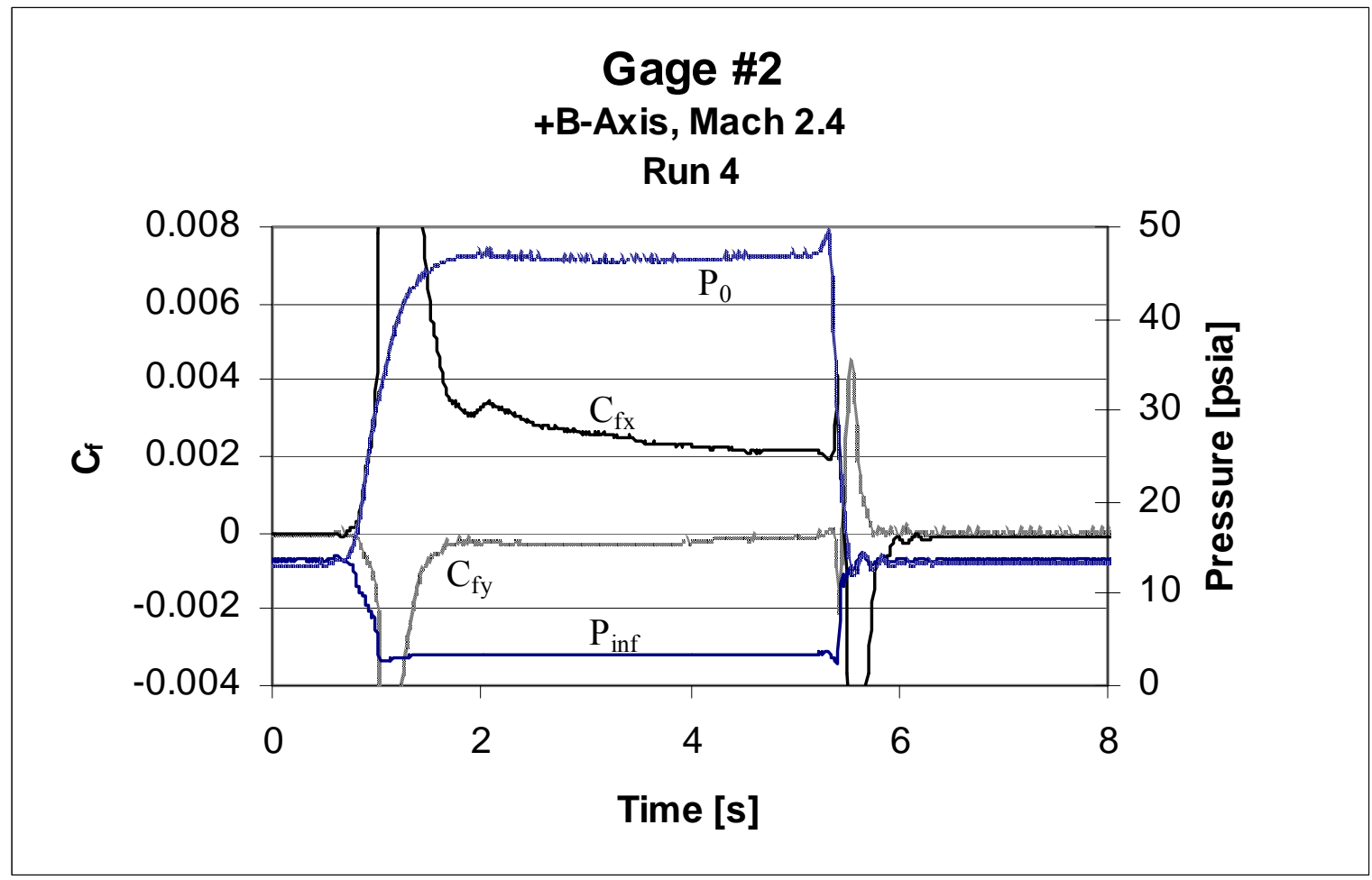

Figure 45: Supersonic Wind Tunnel Test Results: Run 4 
decreased and approached a value of $\mathrm{C}_{\mathrm{f}}$ of just over 0.002. There was no explanation for why the sensor did not read a constant value of $\mathrm{C}_{\mathrm{f}} \approx 0.002$ during these tests.

\subsection{HXEM Tests}

The second skin friction gage design was intended for tests in the Hyper-X Engine Model (HXEM) installed in NASA Langley's Arc-Heated Scramjet Test Facility (AHSTF). The engine model was a full scale, partial width model of the scramjet engine of the Hyper-X flight vehicle. These tests would provide an opportunity to test the skin friction sensor in an environment almost as close to the actual flight environment as could be reproduced on the ground.

\subsubsection{Test Facility}

The facility used for these tests in the HXEM was the NASA Langley AHSTF. This facility is part of Langley's scramjet test complex. The AHSTF can simulate flight Mach numbers of 4.7 to 8 . The primary purpose of the AHSTF is subscale testing of scramjet engines and their components ${ }^{42}$.

A schematic of the facility is shown in Figure 46. The AHSTF is a freejet facility that uses an arc heater to raise the total pressure and enthalpy of dry air. This main air then enters a plenum chamber, where bypass air dilutes the heated air to achieve the desired total enthalpy. The test gas is then expanded through the nozzle. The nozzle exits into a 4-ft $(1.219 \mathrm{~m})$ diameter sealed test cabin, in which the scramjet model is

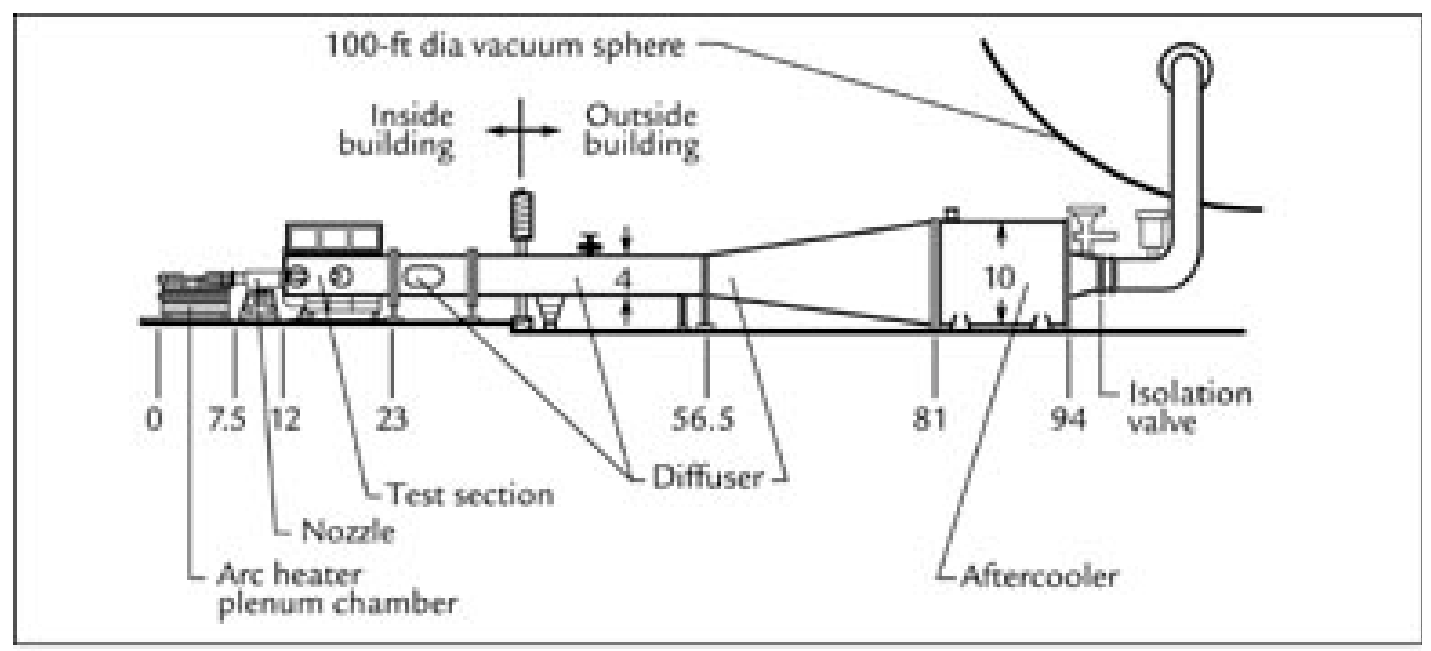

Elevation of the Arc-Heated Scramjer Test Facility heater and plenum chamber. Dimensions in feet.

Figure 46: Schematic of NASA Langley AHSTF ${ }^{42}$ 
mounted on a force balance. After flowing through the test article, the flow enters a 33.5 $\mathrm{ft}$ long straight-pipe diffuser before flowing into a subsonic diffuser. Finally the test gas enters a $100 \mathrm{ft}(30.48 \mathrm{~m})$ diameter vacuum sphere, which provides low pressure in the test cabin $^{42}$.

The arc heater and plenum chamber are illustrated in Figure 47. Two 10 MW DC power supplies provide power to the arc heater. The arc itself can operate at up to 13 MW, delivering up to roughly $6.5 \mathrm{MW}$ to the main air. In this way the total enthalpy of the main air can reach about $3000 \mathrm{Btu} / \mathrm{lb}_{\mathrm{m}}$ with total pressure up to $660 \mathrm{psia}$. The bypass air added in the plenum chamber is then used to regulate the total enthalpy of the test gas to between 500 and $1600 \mathrm{Btu} / \mathrm{lb}$, which corresponds to total temperatures of $2000^{\circ} \mathrm{R}$ $(1111 \mathrm{~K})$ to $5200^{\circ} \mathrm{R}(2889 \mathrm{~K})^{42}$.

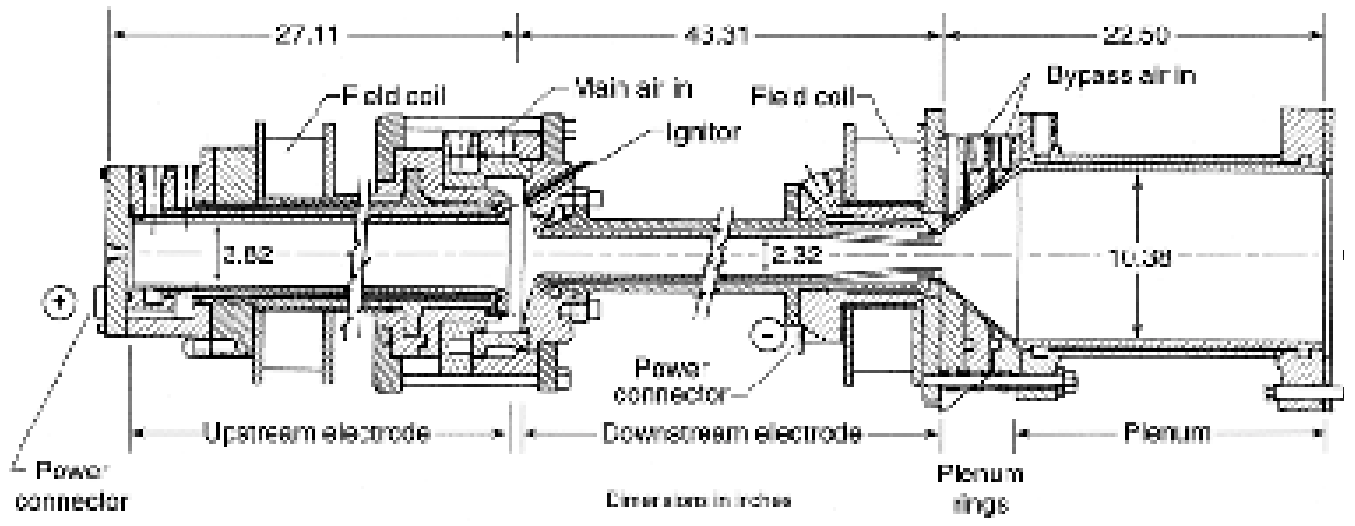

Figure 47: AHSTF Arc Heater and Plenum Chamber $^{42}$

Mach 4.7 and 6 nozzles are available. The AHSTF can simulate flight Mach numbers ranging from 4.7 to 8 . Mach numbers higher than the nozzle exit Mach number are simulated by matching the flight total enthalpy and assuming flight vehicle forebody precompression. This simply means that the test article, if it were installed in an actual flight vehicle, would ingest a flow at a reduced Mach number due to the oblique shocks caused by the forward portions of the vehicle. Therefore the Mach number at the nozzle exit corresponds to the Mach number on the flight vehicle after turning a certain amount. This concept is illustrated in Figure 48. An operational envelope for the AHSTF is shown in Figure 49, where $\alpha$ refers to the associated turning angle of the simulated forebody precompression ${ }^{42}$. 


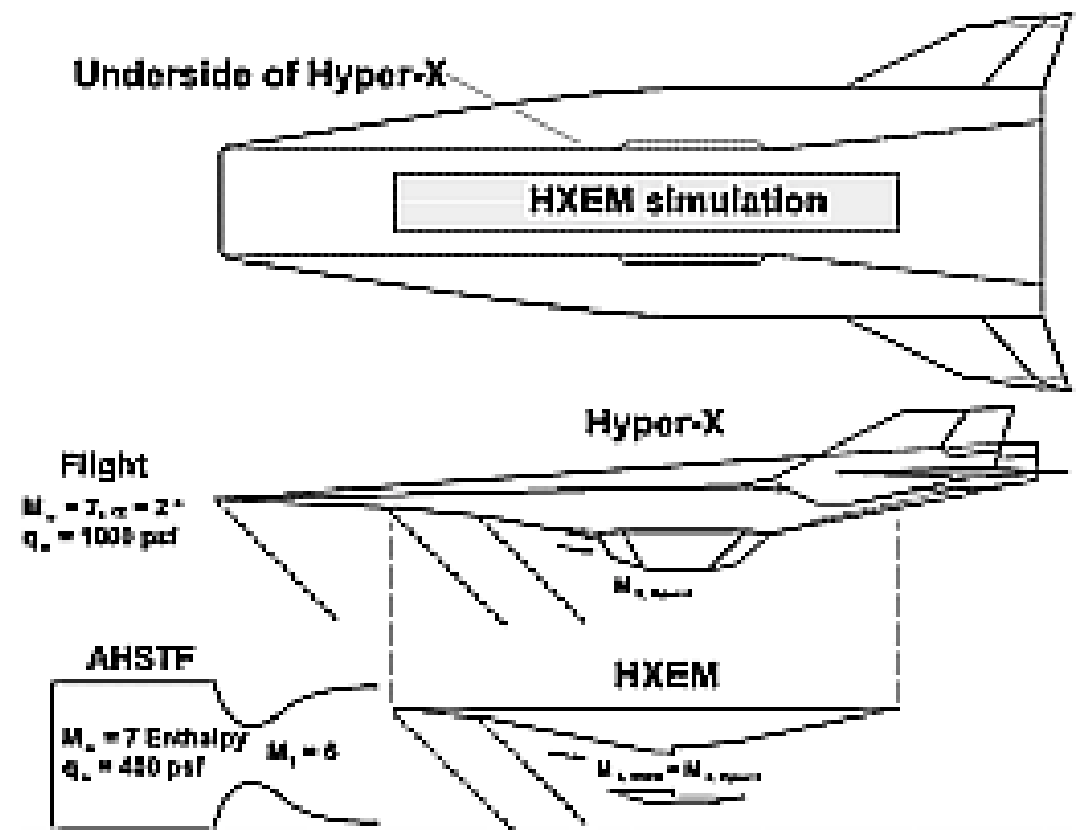

Figure 48: Flight Mach Number Simulation Technique ${ }^{43}$

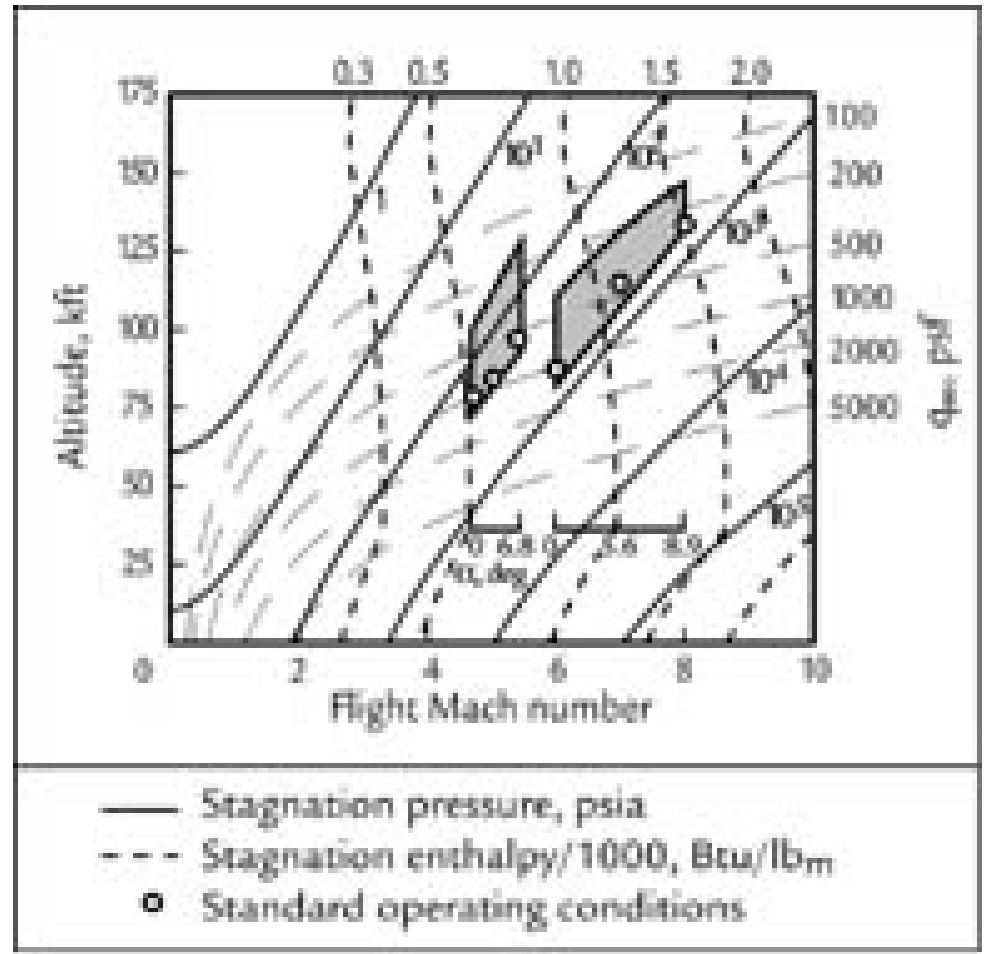

Arc-Heated Scranjed Test Facility Madt number/alitude nap.

Figure 49: AHSTF Operational Envelope ${ }^{42}$ 
For the tests of the second skin friction sensor design, the tunnel was operated at total pressure of about $460 \mathrm{psia}(3172 \mathrm{kPa})$ and total enthalpy of about $1055 \mathrm{Btu} / \mathrm{lb}_{\mathrm{m}}$, $(2450 \mathrm{~kJ} / \mathrm{kg}$ ) corresponding to flight Mach number of roughly 7. The tunnel operation sequence was as follows. The main and bypass air were opened and allowed to stabilize for a couple of seconds. Then the arc power was turned on for a total of about 15 seconds of hot, high enthalpy flow. The bypass air was closed off 5 seconds after the arc power was off, with the main air following about 3.5 seconds later.

A photo of the HXEM installed in the AHSTF test cabin is shown in Figure 50. It was originally hoped to measure the skin friction in the combustor of the HXEM, since that was where the gages were intended to measure skin friction in the flight vehicle. However, the HXEM had been designed and built without the skin friction sensors in mind. With the fuel system, instrumentation, cooling system, and a large motor for opening and closing the cowl, there was no room left for the skin friction sensors to be mounted inside the engine.

There were, however, two locations available for installation of the skin friction gages on the inlet of the HXEM. This is where the skin friction sensors were mounted. The two gages were mounted almost vertically upside down. This created concern about

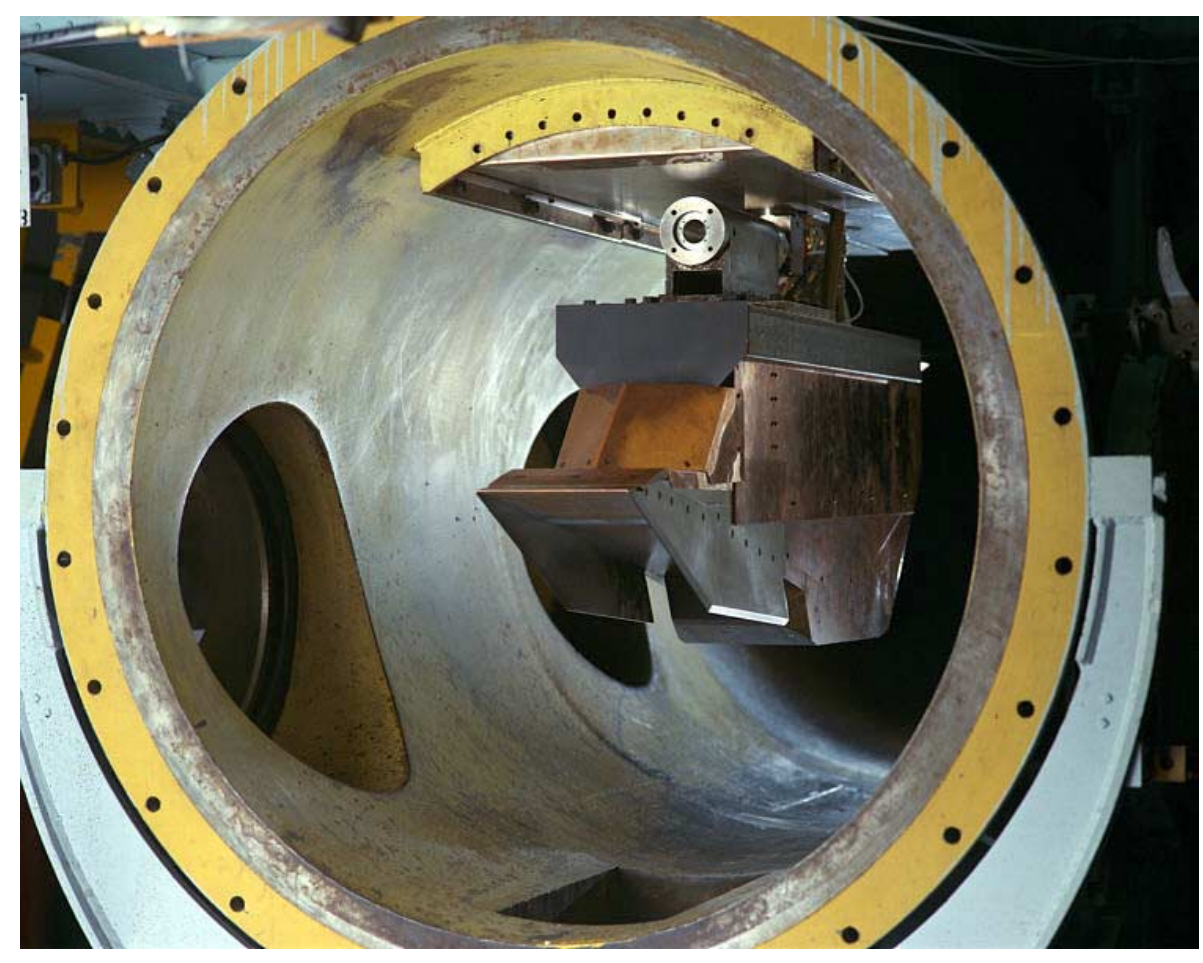

Figure 50: HXEM Installed in AHSTF $^{43}$ 
the oil leaking out of the housing, so the skin friction gages were topped off with oil the morning of testing and installed just prior to sealing of the engine and the test cabin.

Cooling water for the skin friction sensors was supplied from a 125-psi (862 kPa) source. This was routed into the test cabin and split to feed the external cooling lines of the two skin friction sensors. Water from the same source was teed off before entering the test cabin and passed through a pressure regulator to feed the internal cooling lines of the skin friction sensors at a lower pressure.

Data for these tests were recorded on the facility NEFF data acquisition system. The skin friction gages were excited at 5 volts, balanced, and amplified by 1000 by the 2310 signal conditioning amplifiers. For each skin friction gage, two channels of output were acquired from the Wheatstone bridges corresponding to the two axes of measurement. These were sampled at $50 \mathrm{~Hz}$ and filtered at $10 \mathrm{~Hz}$ using the NEFF system analog filter. Each skin friction gage also had a type $\mathrm{K}$ thermocouple, which was inserted through a hole in the base, which measured the temperature inside the housing near the location of the strain gages. These thermocouples made it possible to monitor the temperature of the strain gages to ensure that the cooling system was working and that there was no error due to temperature effects. These signals were also acquired and filtered by the NEFF. Figure 51 illustrates the wiring of one skin friction signal.

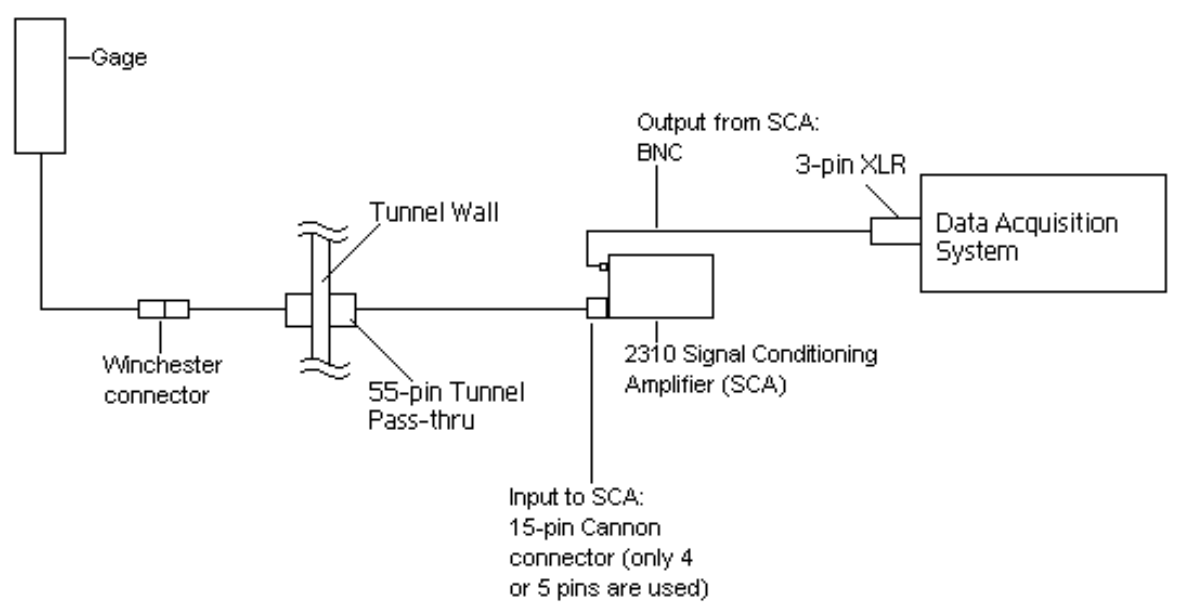

Figure 51: Wiring Diagram for Single Skin Friction Signal in AHSTF 


\subsubsection{Test Results}

Data was recorded for two high enthalpy runs with the skin friction gages installed in the HXEM. About forty seconds worth of data was recorded during each run, with a few seconds before the tunnel came on and several seconds after shut down. The results of the second skin friction sensor design tests in the Langley AHSTF were very disappointing. Results from these tests are shown in Figure 52 and Figure 53.

The total pressure of the wind tunnel, labeled "PT1," is shown to make clear the timing of the tunnel operation. The main and bypass air came on at about four seconds into the data record, raising the total pressure to about $160 \mathrm{psi}(1100 \mathrm{kPa})$. At about 8 seconds, the arc was powered, which led to a jump in total pressure to a level of about $460 \mathrm{psi}(3170 \mathrm{kPa})$. This condition was held until about 20 seconds into the run, when the arc was turned off, allowing the total pressure to fall back to about 160 psi (1100 $\mathrm{kPa}$ ). After several more seconds the bypass air was cut off, causing the total pressure to fall to about $65 \mathrm{psi}(448 \mathrm{kPa})$. At about thirty seconds into the data record the main air was also cut, and the pressure returned to zero. The sequence was the same for both runs, with the exception that in Run 117.01 a malfunction caused the power to the arc to be cut at about 13 seconds into the run, meaning that the high enthalpy portion of the test was cut short. The flow through the wind tunnel without the arc (low temperature and enthalpy) has not been documented. The tunnel condition for this cold flow case is unknown, and therefore little use can be found for data taken during these portions of the tests.

As seen in Figure 52, the output of the skin friction sensors started at zero before the tunnel came on line. The data traces labeled "TAU1A" and "TAU1B" refer to the two axes of one skin friction gage, while "TAU2A" refers to the streamwise axis of the second skin friction gage. The response of the gages to the operation of the wind tunnel was quite erratic. Both sensors gave output that did not follow any sensible pattern during the tunnel operation. Similar results are shown in Figure 53. For this test, the skin friction gages had been removed and switched mounting locations. Clearly, this did not solve the problem. The temperature of the skin friction gages was stable during these tests, as shown in Figure 54. So thermal errors did not appear to be to blame. 


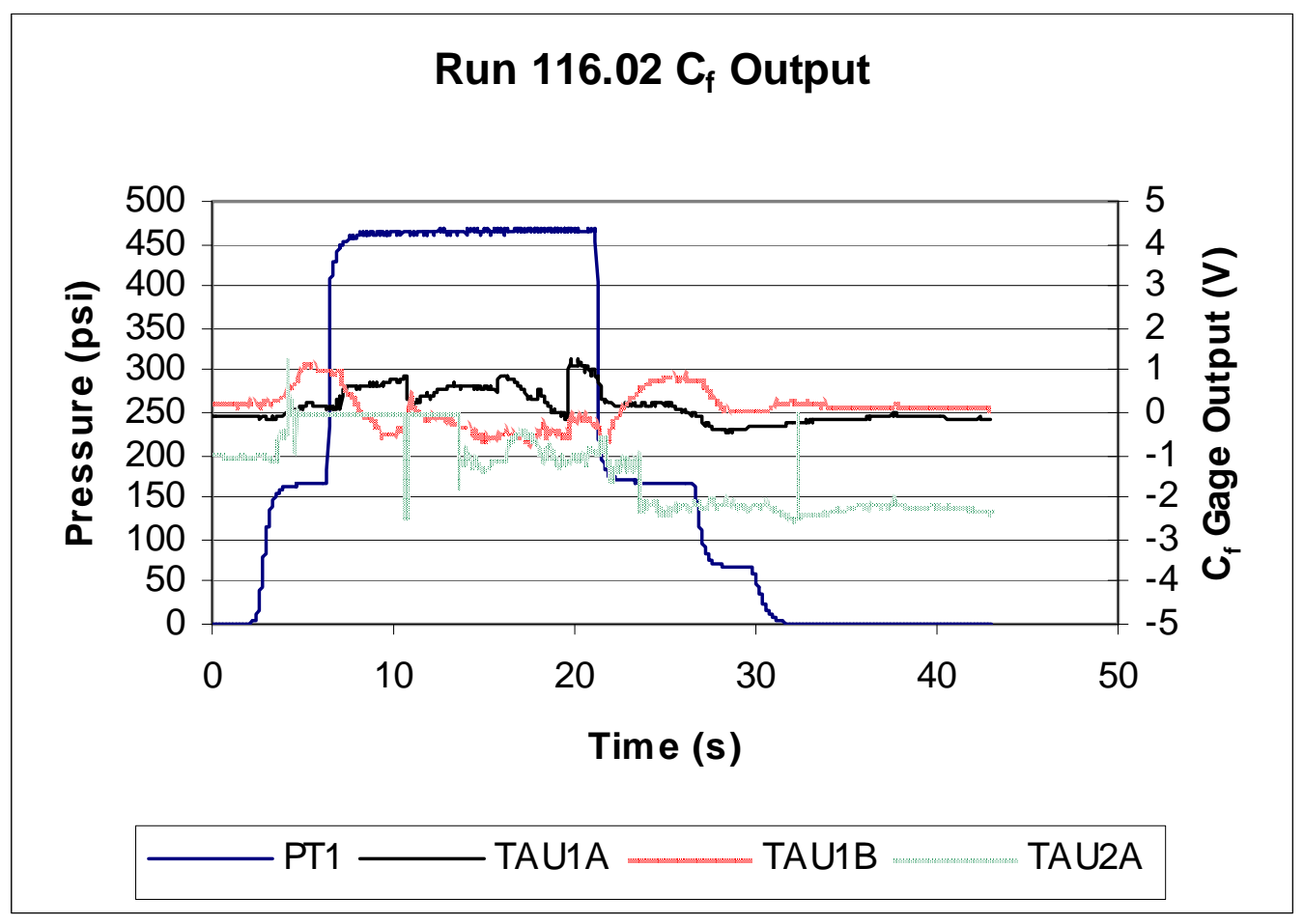

Figure 52: AHSTF Test Results: Run 116.02

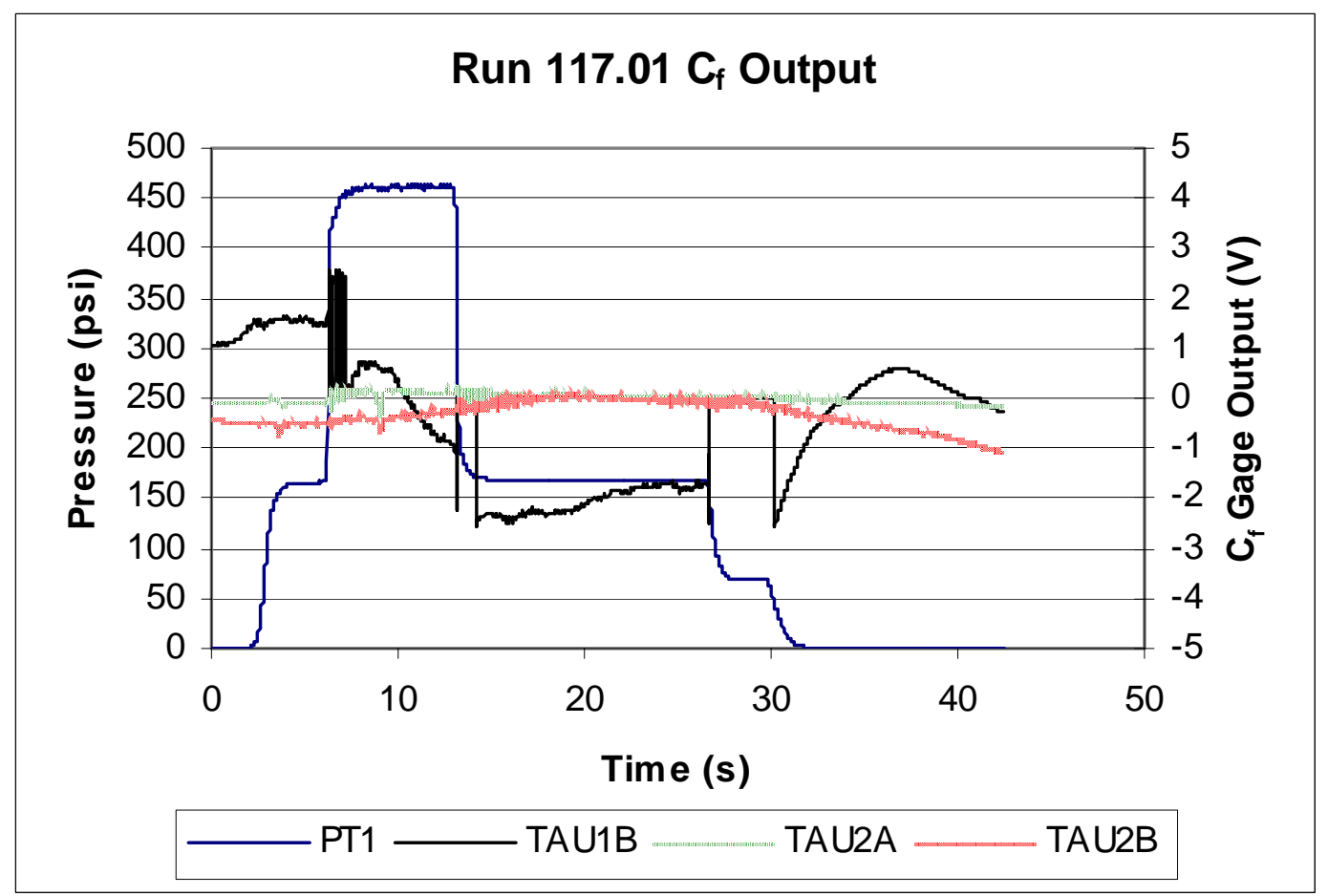

Figure 53: AHSTF Test Results: Run 117.01 


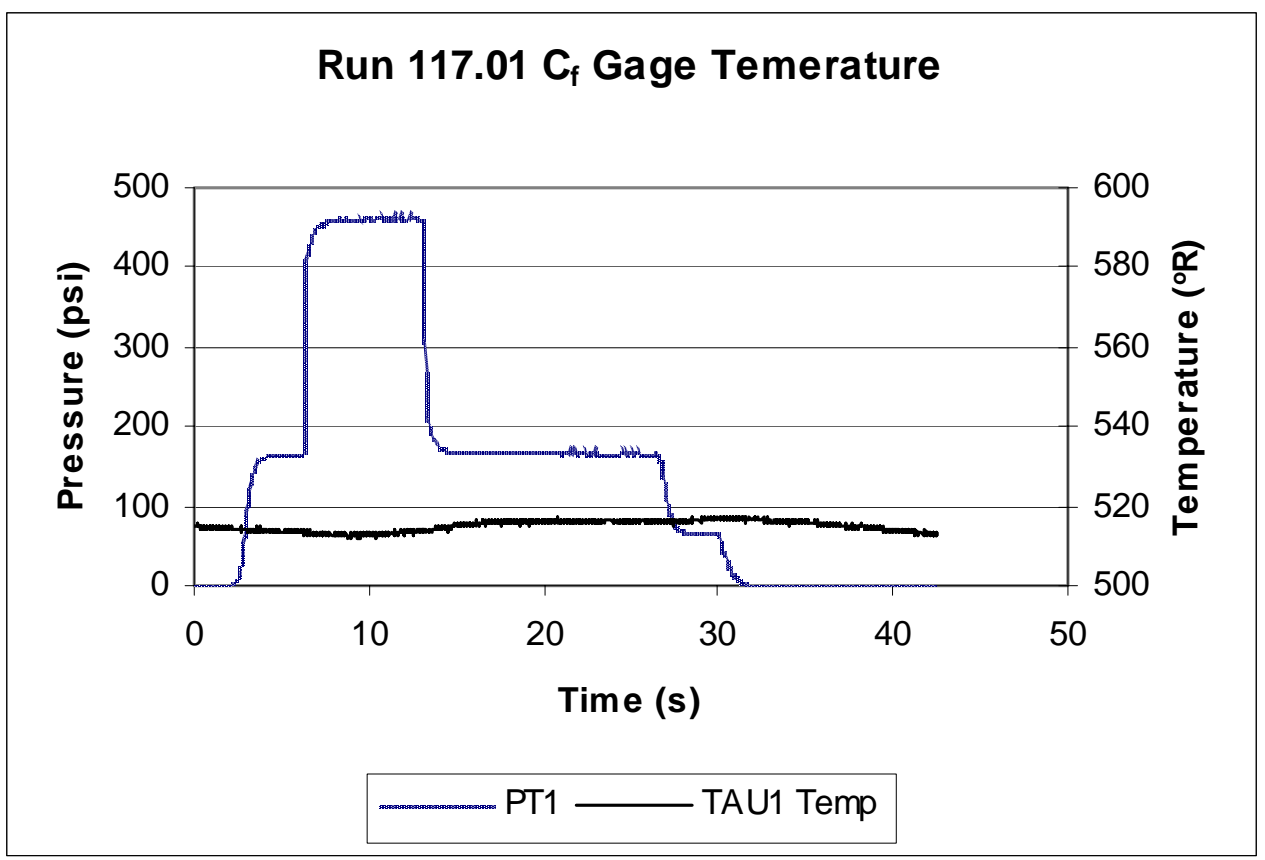

Figure 54: AHSTF Test Results: Run 117.01 Temperature

\subsection{Concluding Remarks Concerning Gage Design Two}

Testing was concluded after only a few runs in the AHSTF because of the malfunction that caused the arc heater to lose power, but also because the skin friction sensors were clearly not working properly. Though much effort was put into making these gages work, several key factors prevented their success.

One problem was the fact that the gages were mounted upside down. It was very difficult to keep these skin friction sensors filled with oil. After testing, when the skin friction gages were being removed from the HXEM inlet, a large amount of oil was observed to have leaked out of the skin friction gages and been smeared along the flow surface. The oil flowing out of the sensors during testing was likely to have been a factor in the bad output. However, another more fundamental problem was also discovered.

Though the skin friction gages built according to the second design behaved well in both the static and dynamic calibrations, there was clearly a problem with the design. This became apparent during the initial internal cooling line checks, the tests in the Virginia Tech supersonic wind tunnel, and in the Langley AHSTF tests.

An important issue was discovered after the supersonic wind tunnel tests, and before the AHSTF tests. It was found that applying even a small force on the internal 
cooling line tubes, which entered and exited through the base of the gage, could cause output from the gage. This was quite worrisome, and efforts were undertaken to strain relieve the internal cooling line tubes. High-temperature, flexible Viton rubber tubing was connected to the copper tubes in hopes that this would prevent forces from being transmitted to the copper tubes. This, however, lead to difficulties in sealing the junctions between the rubber and copper tubing, and small water leaks were hard to eliminate. The water leaked in the AHSTF quickly turned to ice due to the reduced temperature and pressure of the test chamber, making the leaks around the engine model even more of a concern. The rubber tubing didn't completely eliminate the problem of output caused by forces on the tubing either.

After the tests of these gages at NASA were completed, one of the gages was disassembled for inspection. When the beam of this gage was sliced off just above the height of the strain gages, a growing suspicion was confirmed. The internal 0.0625 inch internal cooling tube that ran inside the beam was not perfectly straight, and was in fact hitting the inside of the beam at the same height as the strain gages were located on the outside of the beam. It was then clear that any slight movement of the internal cooling lines or the base would cause this internal tube to transmit that motion or force to the strain gages. This was the source of the extraneous output. Though only one skin friction sensor was disassembled, the same problem must have been true of the other sensors. The clearance between the internal diameter of the beam and outer diameter of the internal tube was simply too small in this design, and contact between the beam and the tube was unavoidable. This design flaw was a major contributor to the failure of skin friction gage Design Two. 


\section{CHAPTER SIX:}

\section{GAGE DESIGN THREE- HYPER-X FLIGHT VEHICLE DESIGN}

The goal of this research was to produce a skin friction gage to be installed on the third Hyper-X Research Vehicle (HXRV), designated X-43A. This flight is to take place sometime in late 2002, and will be the third flight of the Hyper-X test series. During the flight, the vehicle will reach Mach 10 and the scramjet engine will be tested. The skin friction gage discussed below was the final product designed, tested, approved, and installed on the Hyper-X.

\subsection{Gage Design}

\subsubsection{Background to The Design}

The trouble caused by the cooling system of gage Design Two highlighted the fact that it would be even more difficult to incorporate such a system into the gage for the flight vehicle. Since it must be a self-contained entity, the flight vehicle would have even fewer systems and much less space available than the wind tunnel model. There was a cooling system planned for the leading edges of the flight vehicle, but integrating a skin friction sensor into this system would be very difficult, especially since the design of the two entities were taking place in two different geographical locations by different personnel. Plus, the water-cooling lines added risk to the flight vehicle in the event of failure of the cooling lines, as occurred in the AHSTF tests of Design Two.

Also of increased concern was the fact that the gages would have to be mounted upside down in the Hyper-X engine about a year before the actual flight took place. In the AHSTF tests, the skin friction gages gave poor results, and it was thought that oil leakage before and during testing was at least partly to blame. The gages installed on the flight vehicle would be required to remain inverted for far longer before testing, without the prospect of refilling. Some thought was given to methods of keeping oil in the gage for periods up to a year, but ultimately such ideas were discarded.

The complexity of trying to incorporate oil fill and a water-cooling system into a flight vehicle skin friction gage grew, while the chances of these systems actually 
working declined. It was soon realized that the best approach was one of simplicity. So the design of the flight vehicle skin friction sensor was begun with both the oil fill and water-cooling systems deleted from the requirements.

Without cooling water available, it was decided to abandon the semiconductor strain gages in favor of the more thermally insensitive foil strain gages. Though passive methods of thermal protection of the strain gages would still need to be incorporated into the design of the skin friction gage, using foil strain gages would make the temperature sensitivity of the skin friction sensor much more manageable. These strain gages are more common and easier to work with, too. Instead of having to send out parts to have strain gages applied in other parts of the country, personnel within the Aerospace Engineering department were able to perform the bonding and wiring of the strain gages.

Choosing the foil strain gages had a major impact on the design. Though they are much less temperature sensitive, they are also much less sensitive to strain, with typical gage factors of about 2, compared to 150 for semiconductor strain gages. This meant that a much higher amount of strain would need to be induced in the flexible member of the skin friction gage in order to produce a large enough signal from the strain gages. At the same time, the amount of deflection of the floating element would need to remain about the same as the previous designs. Further, size constraints limited the depth the gage could extend into the engine bay. These criteria eliminated the cantilever beam configuration. In its place a very different flexible member design was found.

A skin friction balance designed for tests at the U.S. Naval Ordnance Laboratory in the late 1960's made use of a flexible ring as a frictionless pivot. This balance was a very large, nulling design that used a LVDT and a servomotor to detect deflections and restore the position of the floating element respectively. The flexure ring itself was not instrumented in any way; its only purpose was to deflect under load. The floating element was attached to the flexure ring by a long balance arm, and a counterweight was mounted to the opposite side of the flexure ring ${ }^{44}$.

The flexure ring (see Figures 55 and 56) consists of a ring of material that is connected to a hub at the center of the ring by means of four thin webs. These webs are basically clamped-clamped, or sliding-end beams of rectangular cross section. Equation 21 relates the strain in a clamped-clamped web to a transverse force applied to one end. 


$$
\varepsilon=\frac{P\left(\frac{l}{2}-x\right) \frac{h}{2}}{E I}
$$

\section{Equation 21}

In Equation 21, 1 is the length of the web, $h$ is the thickness of the web, $E$ is the modulus of elasticity of the web material, I is the area moment of inertia of the web cross section, and $\mathrm{x}$ is the longitudinal distance along the web. The strain in the web is a maximum at either end, and is zero in the middle. Equation 22 gives the deflection of the end of the web.

$$
\delta_{\max }=\frac{P l^{3}}{12 E I}
$$

\section{Equation 22}

Unlike the flexure ring that was used in the previous work, it was desired here to instrument the flexure ring itself by placing the foil strain gages on the webs of a flexure ring. Clearly, the strain gages would need to be as close to the end of the web as possible. Since the strain varies linearly over the length of the web, it was easy to develop an equation for the strain that would be measured by a strain gage of finite length by taking the average of the strain at either end of the strain gage. This equation is shown in Equation 23.
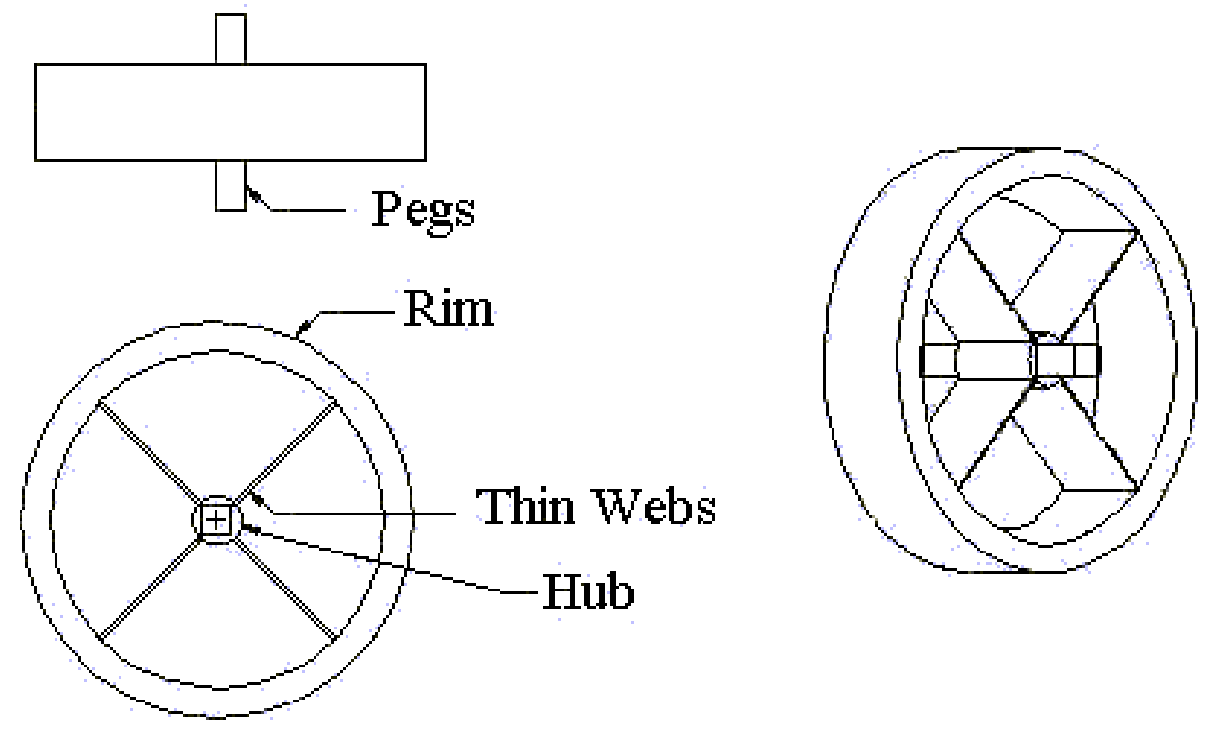

Figure 55: Flexure Ring Concept and Nomenclature 


$$
\varepsilon_{s g}=\frac{P h}{4 E I}\left(l-l_{s g}\right)
$$

\section{Equation 23}

The strain measured by the strain gage is given by $\varepsilon_{\mathrm{sg}}$, and $1_{\mathrm{sg}}$ is the length of the strain gage. For an entire flexure ring in which four such webs are used, the effective force seen by each web is assumed to be $1 / 4$ the total force applied to the edge of the ring.

Using the equations above, an extensive design study was done to find possible flexure ring geometries that would provide a large enough signal from the foil strain gages and would keep the deflection to within a tolerable level. Besides the length, height and thickness of the webs, the method of mounting the floating element was also a variable in the study. Because the floating element might be mounted at a distance further from the center of the hub than the length of the webs, it was possible to increase the effective force seen by the webs due to a longer moment arm.

Early on in the design, it became clear that it would be necessary to be in contact with the designers and manufacturers of the flight vehicle engine in order to ensure that the skin friction sensor would not interfere with other engine components, instrumentation, or other various vehicle systems. In past wind tunnel tests, it had been possible to incorporate skin friction gages into preexisting test articles. In the HXEM engine tests, though, it was not possible to mount the skin friction gages in the combustor

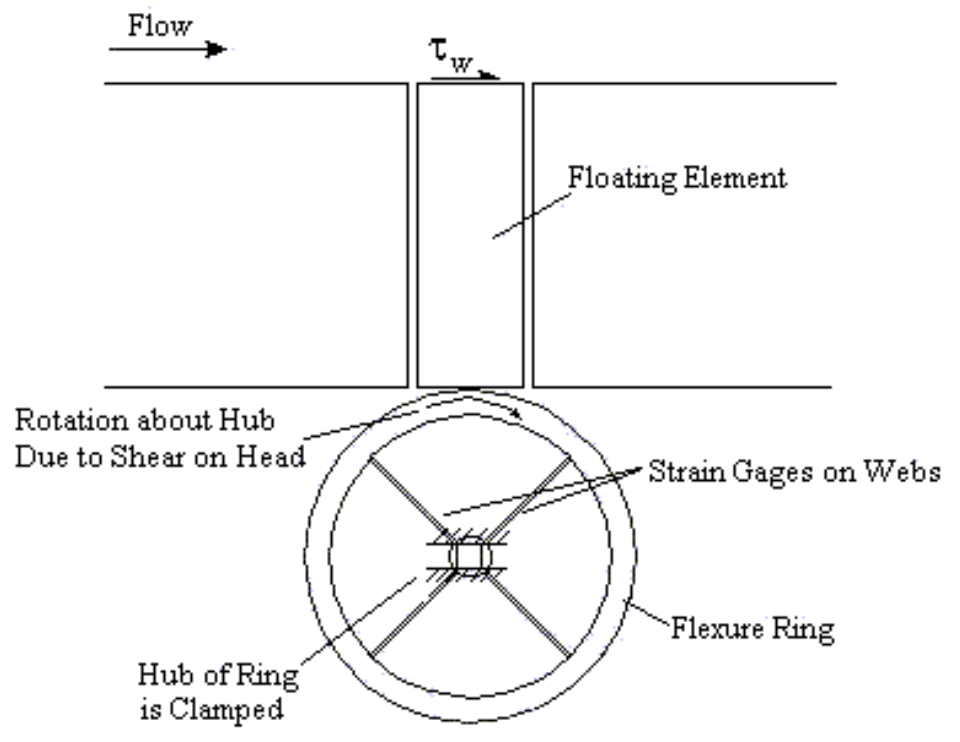

Figure 56: Sketch of a Flexure Ring-based Skin Friction Gage 
because no provision for skin friction gages had been made during the design of that engine. It was certainly necessary to make sure that the skin friction gage for the flight vehicle was integrated into the engine from the earliest stages of design possible.

This meant that possible configurations of the floating element and flexure ring were sent to the engine designers, and based on their feedback, size and shape constraints were added to the design parameters. Also, there were many qualifications and requirements for equipment to be mounted on the Hyper-X flight vehicle. These requirements addressed many different issues, from sealing against hot gas leakage, to vibration qualifications, to electromagnetic interference susceptibility. In particular, the requirement for integrating a flight-qualified electrical connector into the skin friction gage was a major factor in the design.

\subsubsection{Gage Description}

All of the above factors played into the design of the skin friction gage for the

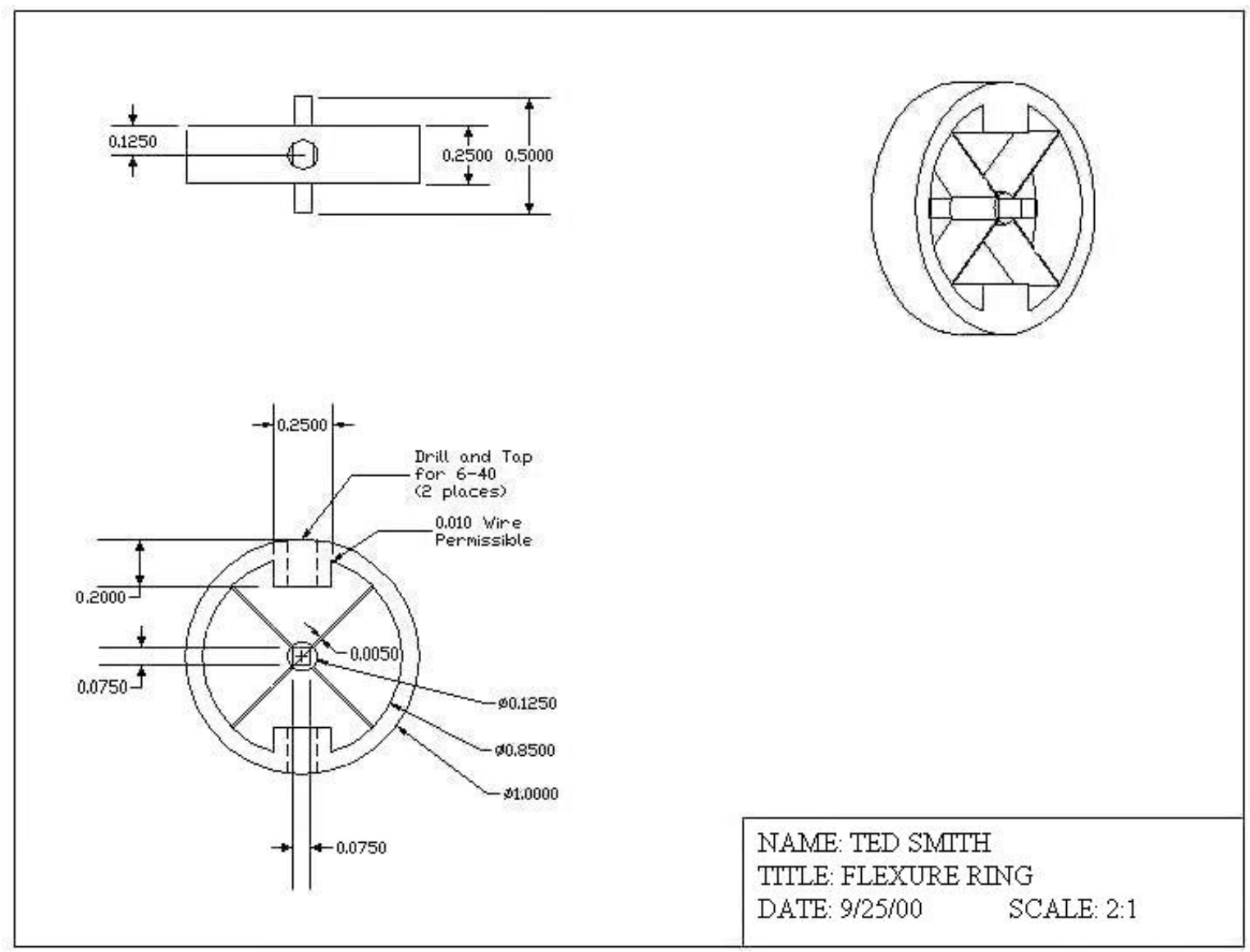

Figure 57: Flexure Ring for Third Design 
Hyper-X flight vehicle. After several iterations, the skin friction gage began to take shape. Figure 57 shows the design of the flexure ring. This was the central aspect of the sensor design, and it was the first part of the skin friction gage to be finalized. The rest of the skin friction gage components were designed around the flexure ring.

The flexure ring was made of aluminum and was machined by an electrical discharge machining (EDM) method. This was necessary due to the very thin webs. The webs were 0.005 in $(0.0127 \mathrm{~cm})$ thick and 0.3625 in $(0.921 \mathrm{~cm})$ long. It would have been extremely difficult to manufacture these webs with conventional machining techniques. The outer diameter of the ring was 1.000 in $(2.54 \mathrm{~cm})$, and the inner diameter was 0.850 in $(2.159 \mathrm{~cm})$. In the center of the ring was a hub of diameter 0.125 in $(0.3175 \mathrm{~cm})$. The ring, webs, and hub were all 0.250 in $(0.635 \mathrm{~cm})$ wide. Two pegs of square cross section extended 0.125 in from either side of the hub. These were used to clamp the ring into the housing. Also, two bosses were machined into the ring on opposite sides. These bosses were drilled and tapped for \#6-40 threads so that the floating element and/or other components could be fastened to the ring.

Figure 58 shows a drawing of the internal components of the skin friction gage. A photo of the actual assembled components with the strain gages mounted on the flexure ring is shown in Figure 59. These components are the flexure ring, the copper floating element, a stainless steel adaptor, and copper counterweight for balancing. The lack of space inside the flight vehicle made it necessary for the flexure ring to be mounted horizontally so as to minimize the total height of the gage. The floating element was then mounted perpendicularly to the ring by means of the stainless steel adaptor. The counterweight was added for inertial damping of the sensing structure.

The adaptor was made of stainless steel to act as a thermal barrier. Stainless steel has a relatively low thermal conductivity when compared to the conductivity of copper (the floating element) or aluminum (the flexure ring). The adaptor is critical since it is directly in the heat-flow path between the floating element, which sees the high heat loads of the flow, and the flexure ring, which has the strain gages mounted on it. Since no active cooling was available for the skin friction sensor, this type of passive thermal protection became even more important. The adaptor was drilled and tapped at the top for a \#6-40 setscrew, which was used to mount the floating element to the adaptor. At 


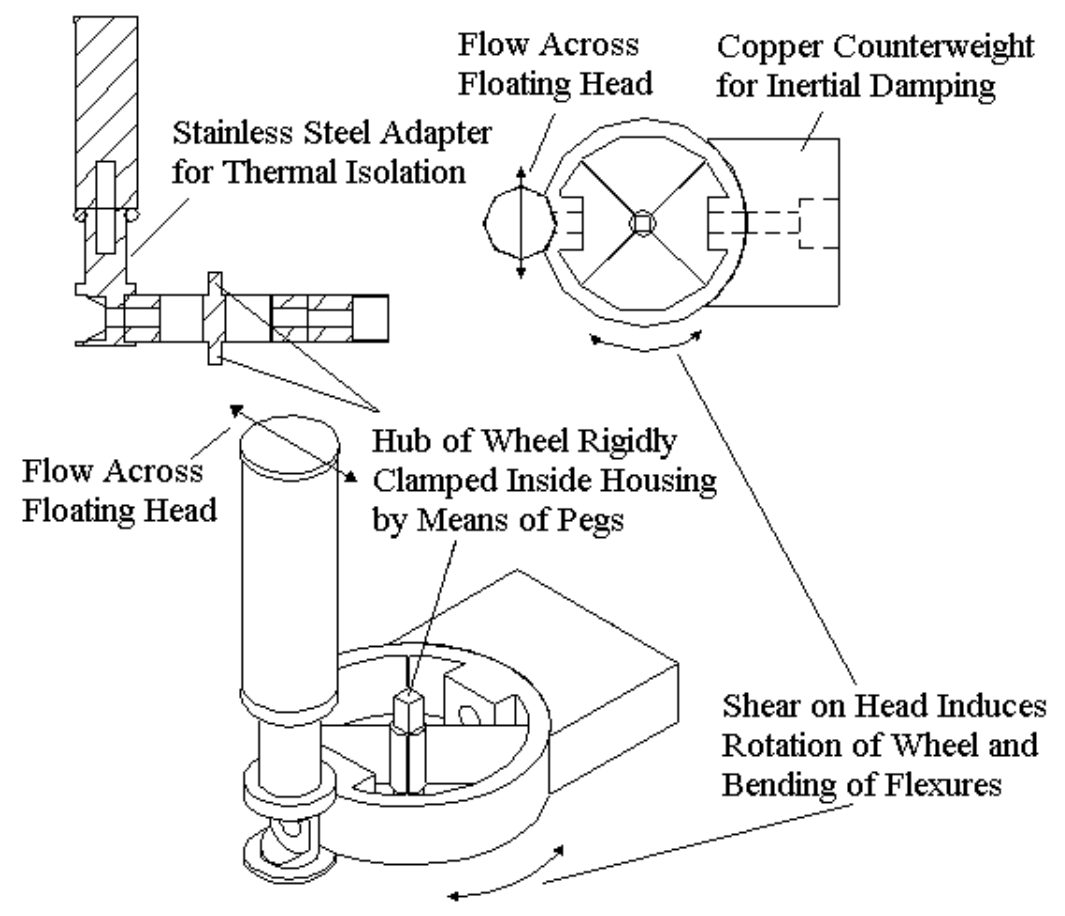

Figure 58: Design Three Internal Components (Head, Adapter, Flexure Ring, Counterweight)

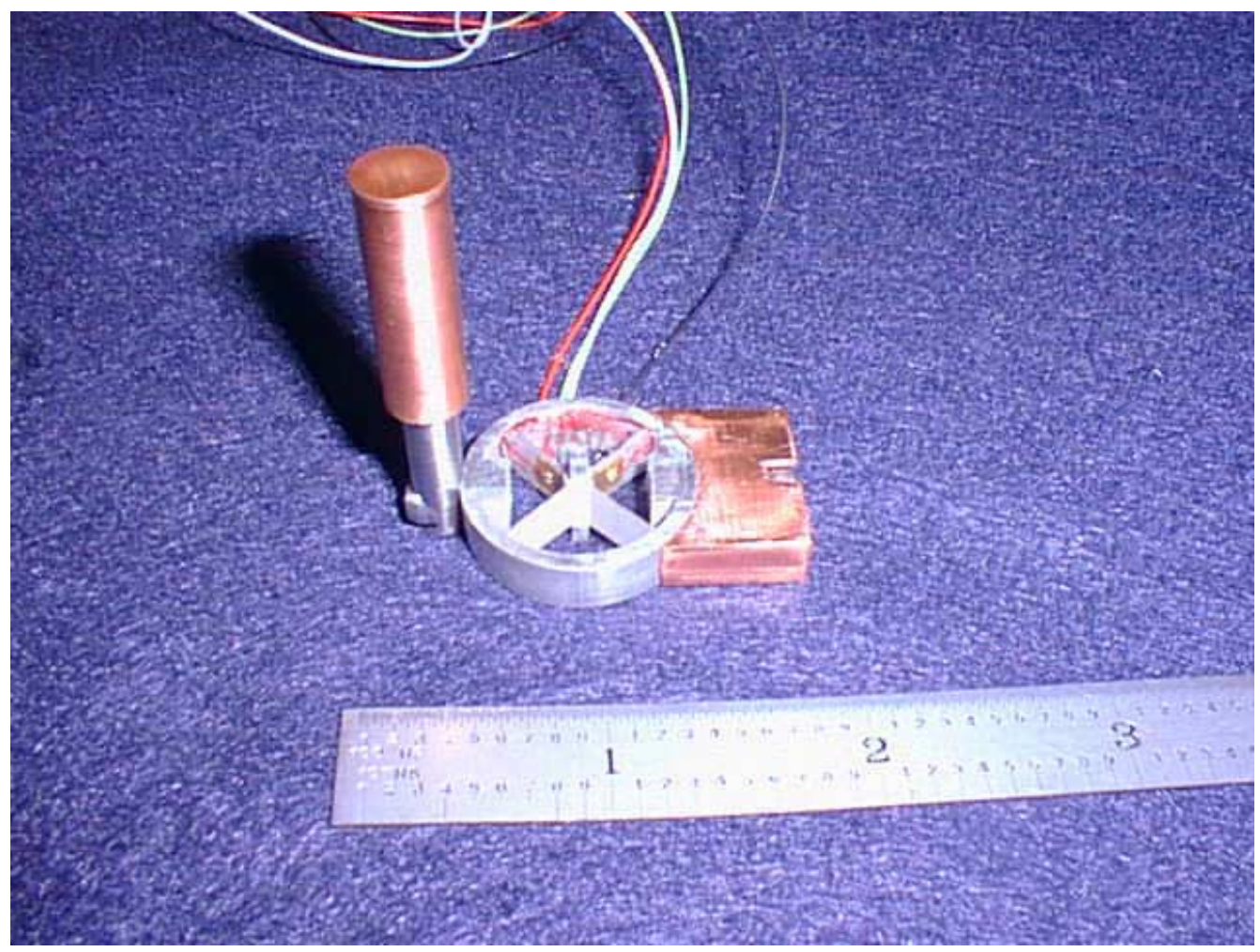

Figure 59: Design Three Internal Components 
the base of the adaptor a clearance hole for another \#6-40 screw was drilled transversely through the shaft. This allowed the adaptor to be fastened to the flexure ring by threading into one of the bosses built into the ring. Just above and below this clearance hole, flanges extended from the adaptor. When the adaptor was threaded onto the flexure ring, these flanges rested against the upper and lower edges of the ring. This prevented the adaptor from being able to rotate around the screw and fixed it perpendicularly with respect to the flexure ring. The adaptor in Figure 59 did not have these flanges and was accidentally tilted during handling. This led to the addition of the flanges to the design, and the adaptor just mentioned was replaced.

A high temperature O-ring was placed over the adaptor and seated against the bottom of the floating element. The outer diameter of the adaptor was machined such that the O-ring would be stretched until its outer diameter was about 0.352 in $(0.894 \mathrm{~cm})$. This is slightly larger than the diameter of the top of the floating element. As was mentioned previously, the housing enclosed the bottom 0.030 in $(0.0762 \mathrm{~cm})$ of the floating element, and so the O-ring also was enclosed. The opening in the housing was the same diameter as the hole in the engine wall, 0.368 in $(0.935 \mathrm{~cm})$. This meant that if the flexure ring should be loaded to high deflections, the O-ring would hit the internal wall of the housing before the floating head would hit the engine wall. This mechanical "stop" was included as a precaution against unexpected loads during testing, improper handling, vibrational loads, etc.

Mounted onto the opposite side of the flexure ring by means of another \#6-40 screw was a copper counterweight. This counterweight was designed to match the masstimes-moment-arm of the floating element and adaptor. This meant that its mass multiplied by the distance from the hub of the flexure ring to its center of mass was equal to the sum of mass times distance from the flexure ring hub of the floating element and adaptor. This type of counter balancing is important in flight tests of force balances such as this skin friction sensor. The skin friction balance design of Lyons incorporated extensive systems to make the balance insensitive to the accelerations that occur on a flight vehicle ${ }^{30}$. That sensor was made to be insensitive to both linear and angular accelerations. The flexure ring only has one degree of freedom, which is a rotation about the hub. The only accelerations that should be of concern are linear accelerations in the 
direction of the flow, and angular accelerations in the plane of the flexure ring. Due to the way in which the skin friction sensor is to be mounted on the flight vehicle, these would correspond to axial force changes (such as due to changes in thrust and/or drag) and yaw rate changes. The counterweight on the flexure ring reduces sensitivity to the linear axial acceleration. The yaw rate changes are assumed to be small during the engine-testing phase of the flight, which is assumed to follow a straight flight path. Therefore, the skin friction gage is expected not to give output due to accelerations on the flight vehicle during the engine test.

By using only one flexure ring in the skin friction sensor, only one axis of motion could be measured. It would be possible to measure two perpendicular directions of flow, but it would require integrating a second flexure ring into the design. Goldfeld, et al, has recently studied a similar scheme for a different type of structure ${ }^{45}$. There was neither enough space in the flight vehicle, nor enough time to develop this more complicated configuration. These skin friction gages were designed to be aligned with the direction of flow, which should be mostly 2-D. This configuration allowed the most important component of skin friction to be measured in the engine of a scramjet flight vehicle, something never before done, and remained simple enough to build and test.

Figure 60 shows all of the skin friction gage components in an exploded view, and Figure 61 shows a print of the assembled gage with dimensions. The housing was made of brass and was designed in two pieces to fit around the internal components. A six-pin connector was integrated into the design, and fastened to a large extension on the side of the housing. High temperature NBR gaskets were used to seal between the two housing pieces (gasket 1 in Figure 60), and between the housing and the connector (gasket 2). These gaskets are rated to $400^{\circ} \mathrm{F}$ continuous, and up to $700^{\circ} \mathrm{F}$ for short periods of time. A third gasket, made of high temperature graphite, was used to seal between the upper surface of the housing and the engine wall (gasket 3). This material was rated up to $975^{\circ} \mathrm{F}$. Two dowel pins were used to align the two housing pieces with each other, and two more dowel pins were used to align the skin friction sensor in the engine wall. 


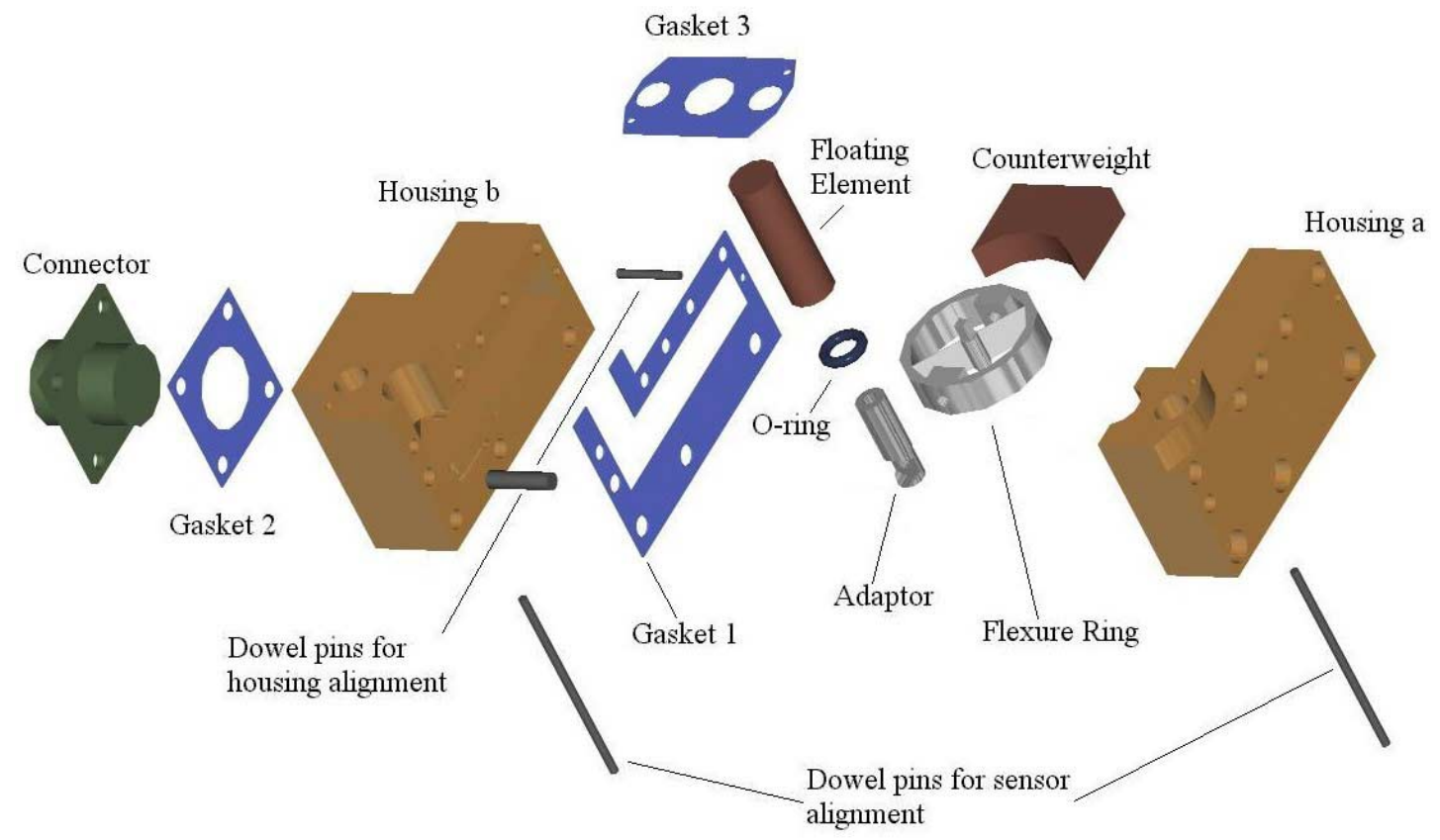

Figure 60: Design Three Exploded View

The housing was made of brass partly because of its relatively low thermal conductivity, but also for it ease of machining. The housing was very complicated and had many machined features. The upper portion of the housing was made with the same flange and clearance holes for $\# 1 / 4-20$ mounting screws as the two previous skin friction sensor designs. The overall shape of the housing, however, was vastly different from the earlier designs. As mentioned previously, the housing was formed from two pieces. The slice between the two pieces was not cut in line with the center of the flexure ring, but was offset a distance equal to the thickness of the pegs that extend from the hub of the flexure ring. The piece labeled as "Housing b" in Figure 60 was the larger of the two. Rectangular cutouts were machined into "Housing b" so that these pegs would fit into them. \#0-80 threads were drilled and tapped vertically into "Housing b" so that sharpened setscrews could be tightened against the flexure ring pegs. This was the method by which the internal components were mounted into the housing. The first gasket was then laid over the internal components, and "Housing a" was then guided onto the dowel pins and tightened down with several socket head screws. 


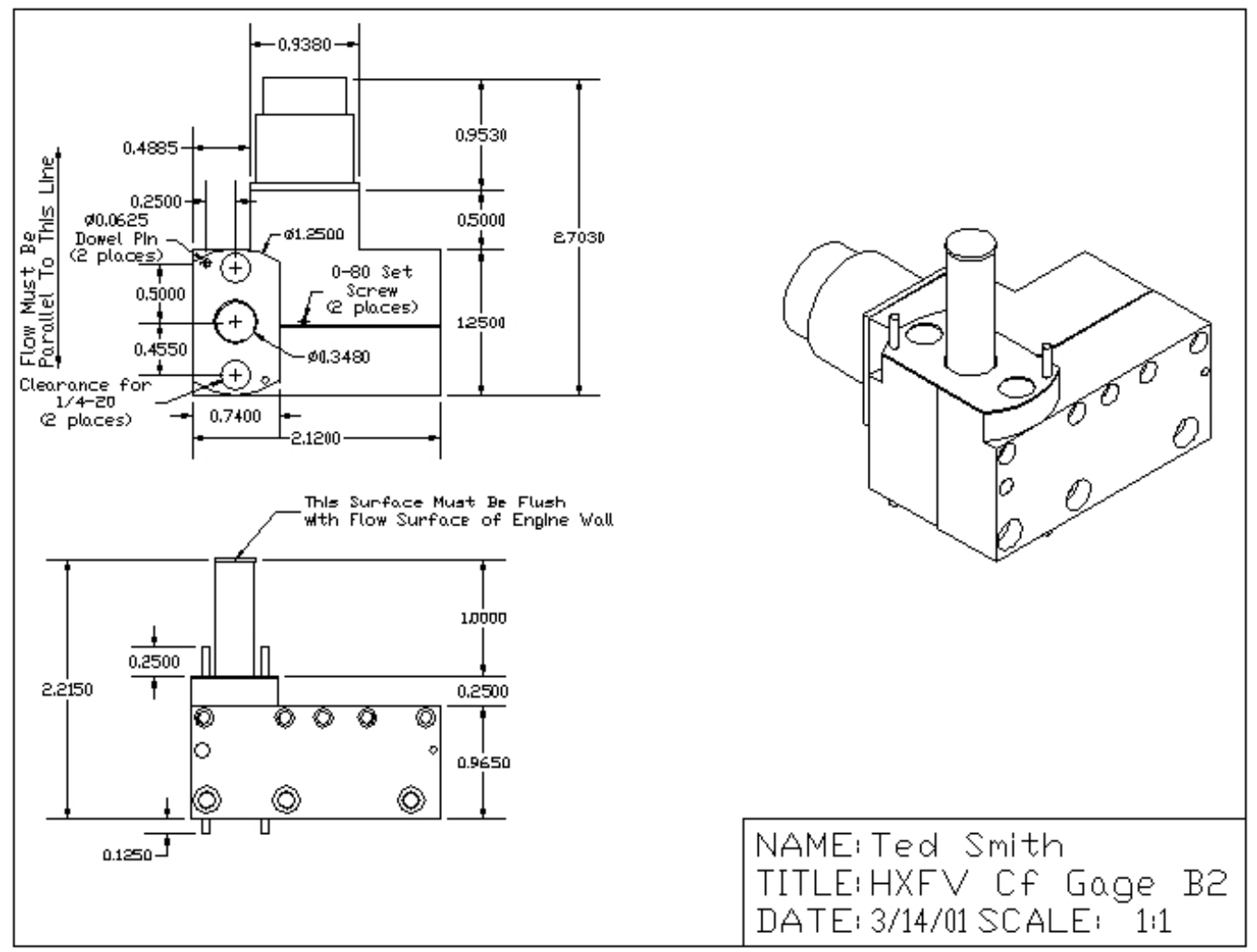

Figure 61: Design Three Print

The internal components were assembled such that the wire leads from the strain gages were on the side of the flexure ring that was within "Housing b." These leads were routed through an opening in the wall into the large extension on the side of "Housing b." This extension had a circular cutout to fit the connector. The leads were routed to the connector, and then the connector was mounted onto the extension on "Housing b." Figure 62 shows how the internal components were mounted into "Housing b."

A Type $\mathrm{K}$ thermocouple was installed in "Housing b" just below the location of the adaptor. A groove was machined into the surface of the housing piece and the thermocouple was laid in this groove. Two-part epoxy was used to secure the thermocouple in place. After the epoxy cured, it was sanded so as to be flush with the rest of the surface of the housing. This was important since it was the surface between the two halves of the housing, and good sealing by means of the gasket was necessary. The thermocouple groove can be seen in the surface of the housing below the adaptor in Figure 62. The leads from the thermocouple were also routed into the extension by means of a hole. 

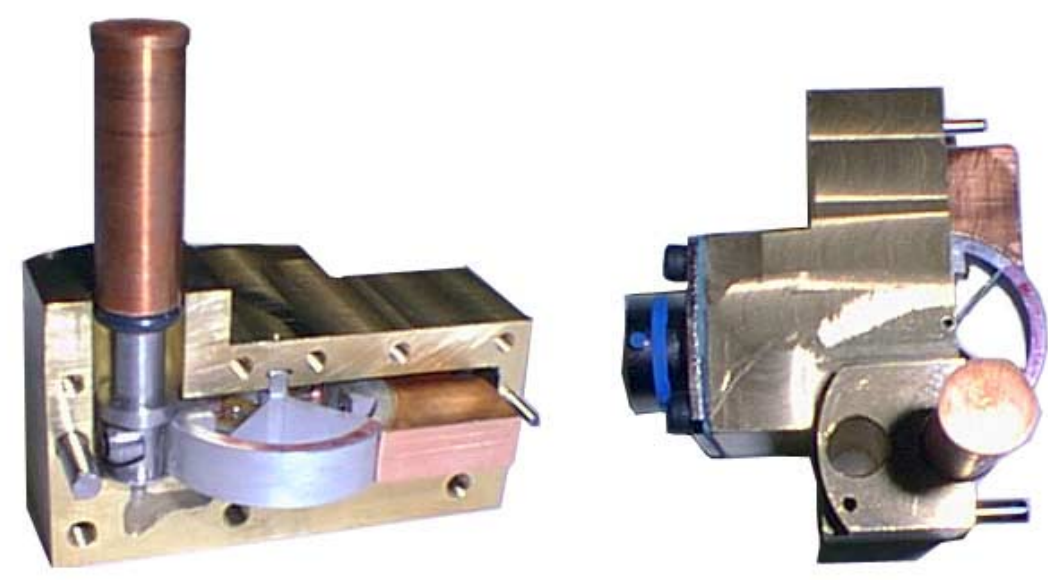

Figure 62: Design Three Internal Components Mounted in "Housing b"

The connector that was used was a high temperature, aerospace rated connector. It was desired that one connector that could carry the leads from both the strain gages and the thermocouple be obtained. The smallest such high-temperature connector to be found was military specification MS27499E10F98P, which has provisions for six crimp-type pins, labeled A, B, C, D, E, and F. Pins were ordered separately so that they could be crimped to the appropriate leads and inserted into the connector. Pins for A, B, C, and D were used for the four conductors of the Wheatstone bridge, while pins for $\mathrm{E}$ and $\mathrm{F}$ were Type $\mathrm{K}$ thermocouple pins. These pins are specially made to match the materials of the different thermocouple leads. These were crimped to the lead wires from the Type $\mathrm{K}$ thermocouple located inside the housing of the gage. Pin E was connected to the chromel lead, and Pin F was connected to the alumel lead. A corresponding plug and crimp type sockets were obtained to go with the connector. The plug is military specification MS27473T10F98S, and strain relief MS27506F10-2 was added.

During assembly of the first of these skin friction sensors, it was found that it was important to strain relieve the wires inside the extension on "Housing b." The lead wires from the strain gages were relatively stiff compared the webs of the flexure ring. When the connector was mounted onto the housing, these wires were tightly bundled into the space inside the extension. During calibrations of the gage after this procedure, decreased sensitivity and increased hysteresis were observed. This problem was solved by adding length to the extension, thus increasing the distance between the connector and 


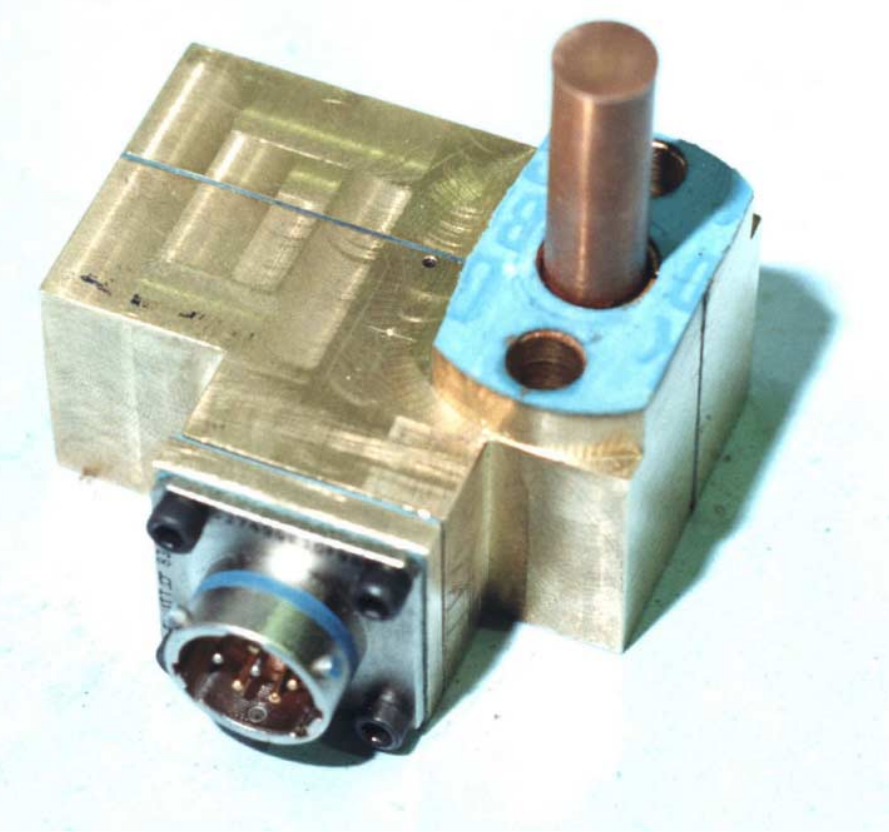

Figure 63: Design Three Final Assembly

the flexure ring, and increasing the space inside the extension for the lead wires to be strain relieved.

Figure 63 shows a photo of one of the two completed skin friction sensors. (The graphite gasket material later replaced the blue NBR gasket seen on the top of the housing in the picture.) The requirements for making a skin friction gage that was suitable for flight-testing on the Hyper-X led to a design that was quite different from any previous skin friction sensor. This becomes quite clear when comparing Figure 63 to Figure 20 and Figure 41. As is true of most all skin friction sensors, the specific application drove the design. Though some aspects of the design were drawn from previous sensors, those features have never been combined in the same fashion. The final result stands as a unique instrument, designed to meet very rigorous requirements.

\subsection{Calibration Results}

Even before the skin friction sensor had been completely designed, tests were done on the flexure rings by themselves. There was some concern as to whether the strain in the webs would remain linear in the range of loads expected. Also, though the webs are much more flexible in the direction of rotation about the hub, it was desirable to determine the sensitivity of the flexure ring to loads in directions other than the design load. These tests were done using the static calibration technique described earlier, but 
the loads were hung directly from the edge of the ring since no floating head was used. Linearity of the flexure ring was found to be very good. Weights were then applied transversely with respect to the plane of the ring, and also radially in the plane of the ring. Sensitivities in these directions were found to be less than $1 \%$ of the sensitivity in the design loading direction (rotation about the hub).

Since these sensors used foil strain gages, they were much less temperature sensitive. This also meant that a higher excitation could be used in all of the testing done. The previous sensors were excited at 5 volts, and a 10-volt excitation caused excessive drift in their semiconductor strain gages due to self-heating. Thermal output could even be induced in the semiconductor strain gages by simply placing one's hand within an inch of them. The third design sensors proved to be much more thermally stable. The 10-volt excitation was used with no problem. Overall, there was less drift in the output from these sensors during calibrations or over periods as long as several days. In general, the foil strain gages were much easier to work with and better behaved than the semiconductor strain gages of the previous designs.

Once completed and assembled, the static calibration method was used to calibrate the skin friction gages designed for the Hyper- $\mathrm{X}$ flight vehicle. These sensors were not calibrated using the glycerin flow calibration rig. Since these gages did not have oil filling the interior space, it was feared that the glycerin would enter into the housing. It would have been very time consuming to then disassemble and remove the glycerin from the gages. The previous experience with the calibration rig showed that it gave the same results as the static calibration, so it was not deemed necessary.

Figure 64 shows the results of a static calibration of one of these skin friction sensors. As just mentioned, the excitation was set at 10 volts. The amplification factor was reduced from that which was used for the earlier designs, from 1000 to 500. This meant that the sensitivities of the different designs could still be directly compared. The excitation had been doubled, but the gain had been halved. The linear fit to the data shown in Figure 64 indicates a slope of roughly 0.16 volts/gram. This compares to about 0.72 volts/gram for Design One, and about 0.59 volts/gram for Design Two. Clearly, some sensitivity was sacrificed by using the foil strain gages, even with the flexure ring design. The linearity of the output was very good, and there was a very minor amount of 


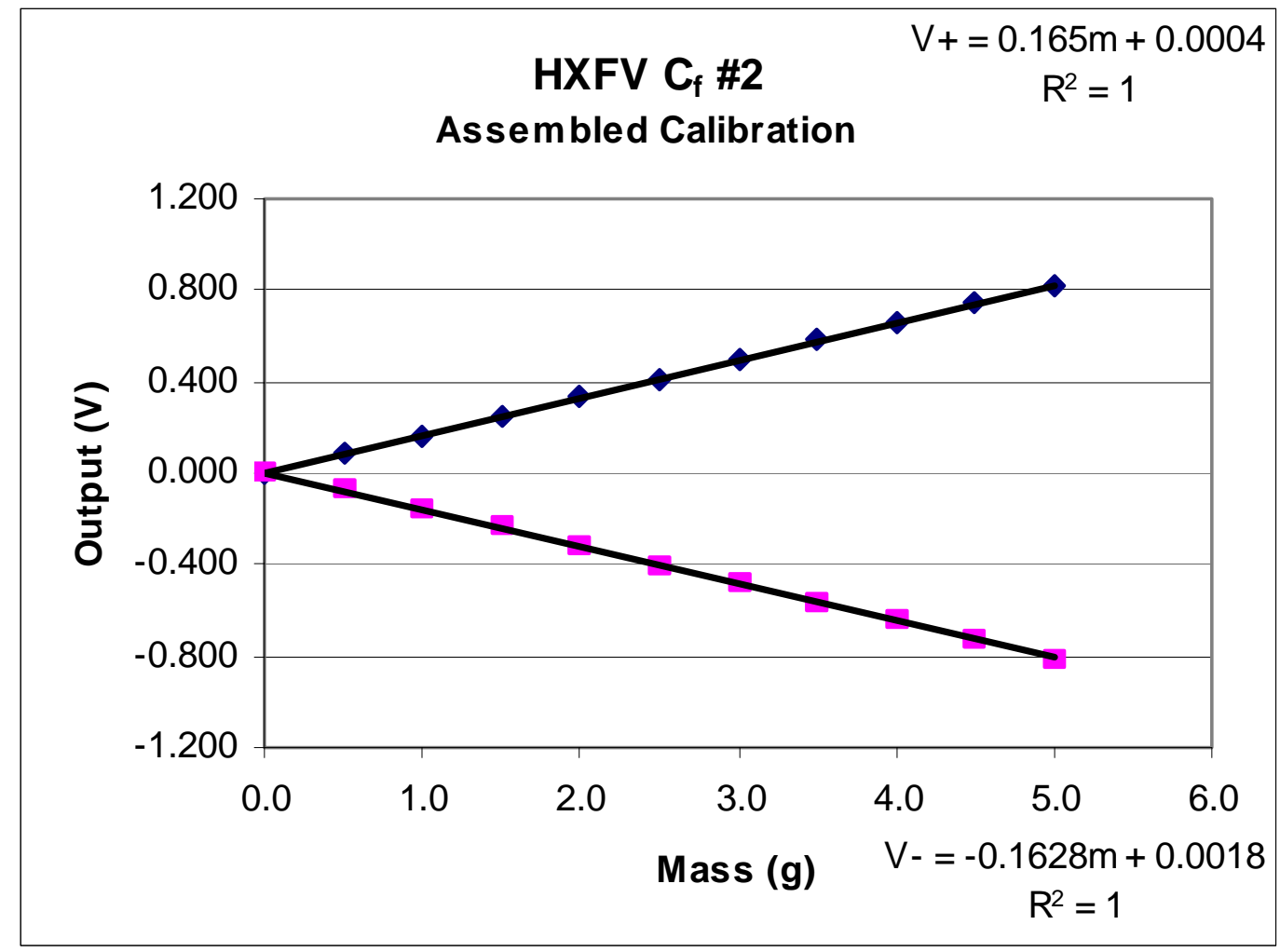

Figure 64: Sample Calibration For Design Three

hysteresis. As seen in Figure 64, calibrations in both the positive and negative directions gave similar sensitivities.

Since this gage was to be mounted on the Hyper-X Flight Vehicle (HXFV), and the vehicle would go be oriented at different angles during its flight, the gage was calibrated to determine any output due to angle changes. Such output could be caused by the changes in the gravity vector loading of the floating head mass. The gage was to be mounted in the vehicle such that the axis of the aircraft was aligned with the direction of motion of the floating element. This meant that changes in the pitch angle, or angle of attack, of the aircraft would cause the component of the gravity vector in the axial direction to change. This was the primary concern that warranted these tests, but the sensitivity to roll changes was also calibrated. Yaw angles cannot cause output from the sensor since they are about the same axis as the flexure ring hub.

Figure 65 shows the orientation and notation of these angles. Results of these angular calibrations are shown in Figure 66. The output due to changes in pitch angle (angle of attack) was small, about $40 \mathrm{mV}$ maximum. This was because of the 
counterweight. Theoretically, if the counterweight had been perfectly matched, there would have been no output at all. Though it was not possible to make a perfect counterweight, the output was satisfactorily small. The maximum output due to roll was about $55 \mathrm{mV}$. Also of note is that the output was greatest for large angular excursions from straight and level flight. If the output from the Wheatstone bridge is balanced before the flight when the vehicle is horizontal (zero pitch angle) and level (zero roll angle), then the gage output due to pitch and roll angles should be small. This calibration, however, makes it possible to subtract out the output due to these angles, should it be necessary.

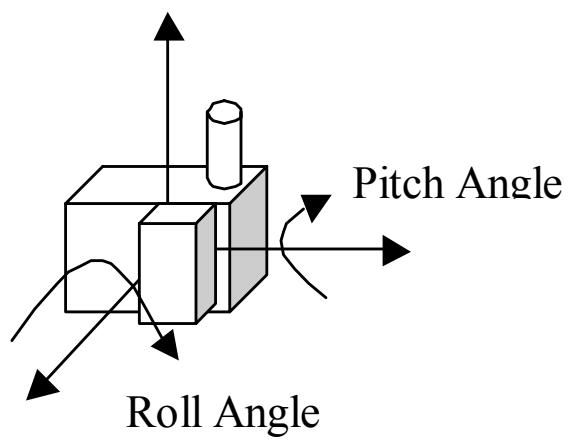

Figure 65: Angular Calibration Notation

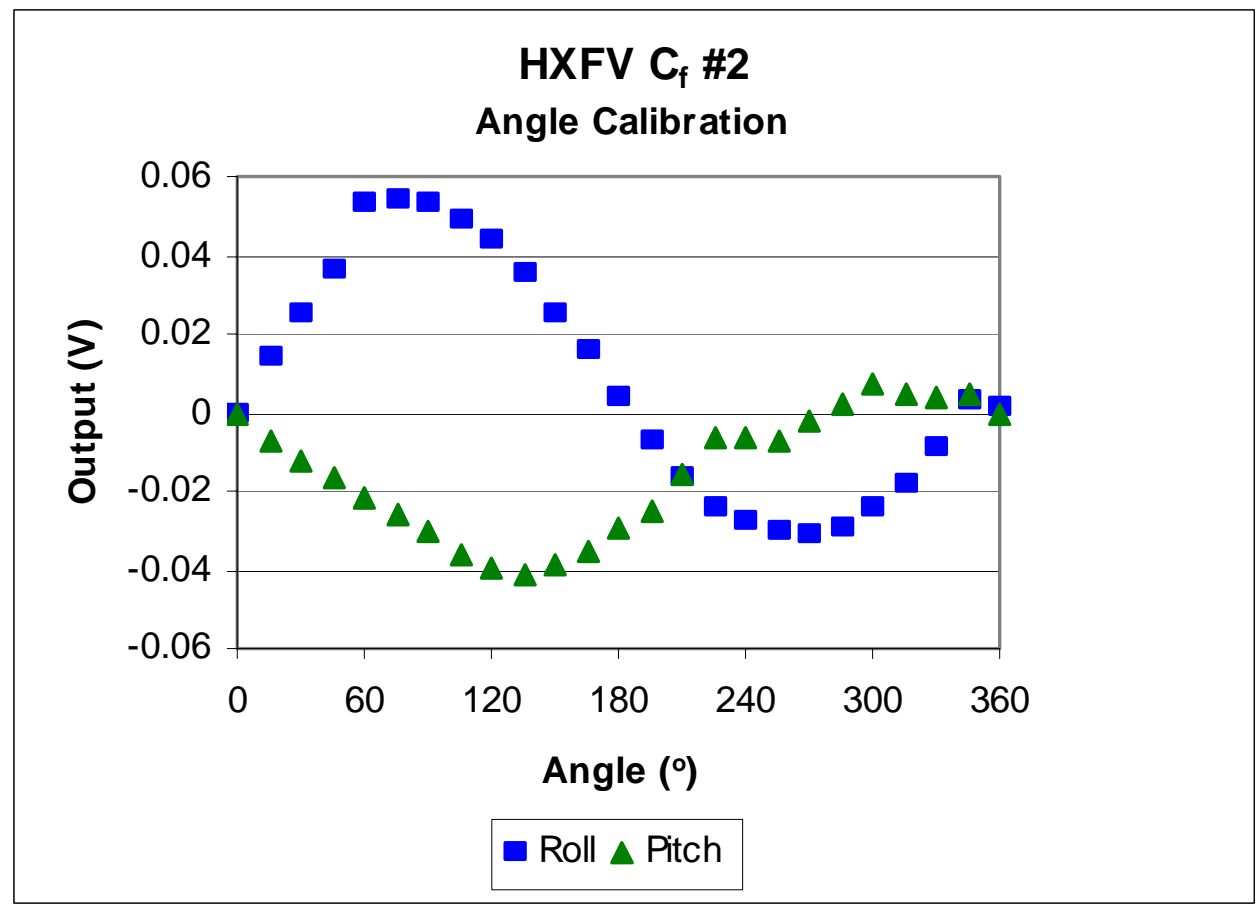

Figure 66: Angular Calibration Output 


\subsection{Vibration Tests}

The skin friction gages for the Hyper-X flight vehicle were subjected to vibrational tests. These tests were used not only to determine the natural frequencies of the sensors, but also to qualify them for the flight vehicle environment. For the Hyper-X flight test, NASA provided a list of qualification tests that all equipment to be installed on the vehicle needed to pass. Among these were tests to verify the ability of such equipment to withstand the estimated vibrational environment of the flight test.

In order to meet these requirements, the skin friction sensors were shaken in the Virginia Tech Modal Analysis Lab. These tests were similar to those discussed previously. The skin friction gage was fastened to a shaker (Vibration Test Systems VG 100-41), and an accelerometer (Kistler 8630B50) was mounted onto the skin friction gage. As usual, the 2310 signal-conditioning amplifier powered the skin friction gage. An HP 35665A Digital Signal Analyzer was used to supply the source signal for the shaker and to acquire and process the signals from the accelerometer and the skin friction gage. An Onkyo Integra M-504 stereo power amplifier amplified the source signal from the DSA. The test setup is shown in Figure 67.

The first test was the test of survivability. Since the flexure ring was the only moving part in the sensor, it was the part that had the greatest possibility of failure. Therefore, a skin friction sensor was assembled with a flexure ring that had no strain

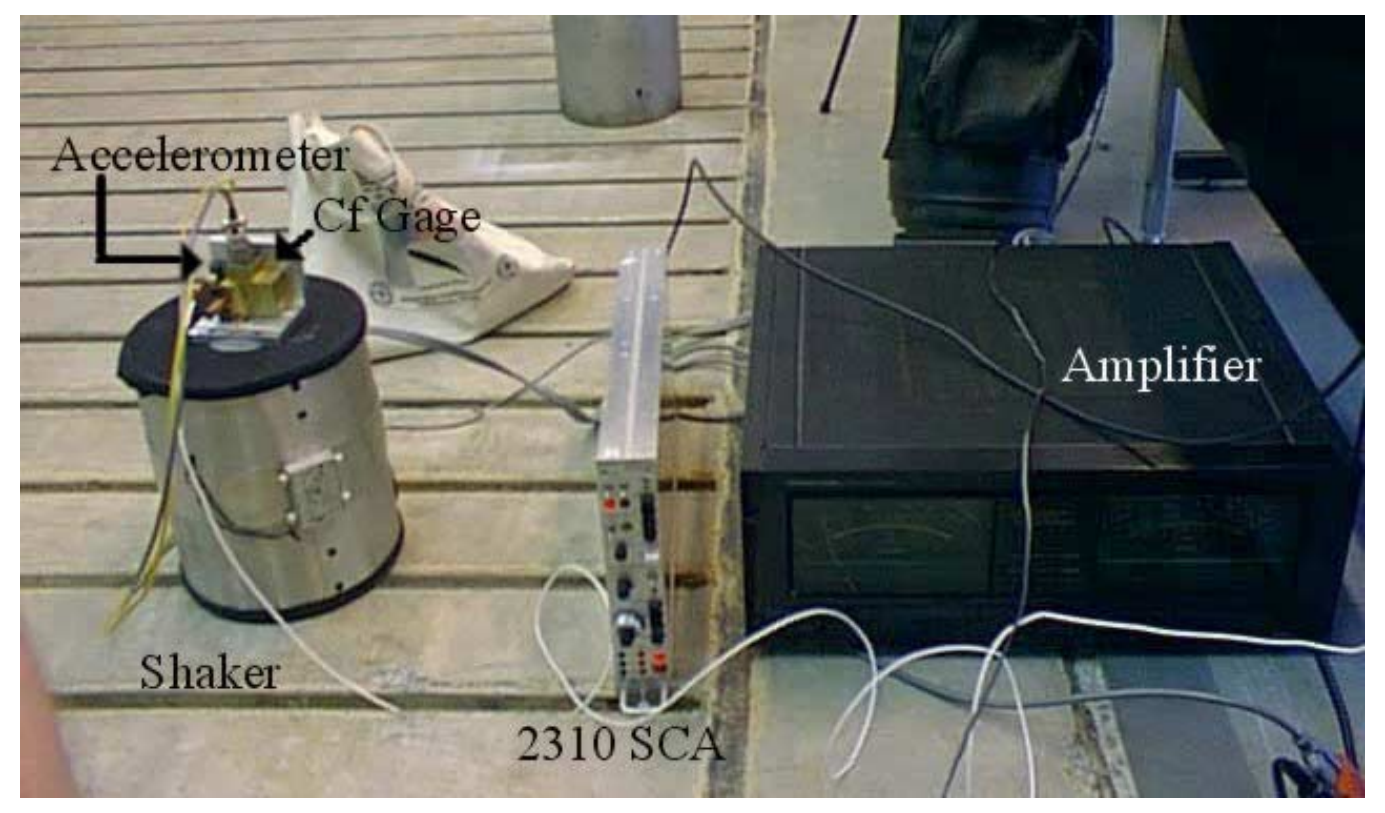

Figure 67: Vibration Test Setup 
gages mounted onto it. In case the flexure ring did fail, it would not be one that had already been instrumented with strain gages. This dummy skin friction gage was shaken with a sine wave at frequencies of $15,100,150,300,500,1000,1500$, and $2000 \mathrm{~Hz}$ at levels of 2 and $8 \mathrm{~g}_{\mathrm{rms}}$. This was done along all three geometric axes. The total time shaken along each axis at $8 \mathrm{~g}_{\mathrm{rms}}$ was roughly 6.5 minutes.

Before the above tests were done, a failure mode test was done. It was discovered that at extremely high g-loads (20-50 $\left.\mathrm{g}_{\mathrm{rms}}\right)$ a vibrational mode could be excited that could lead to the failure of the ring, but only if the floating head was not constrained. In this manner a non-instrumented flexure ring was shaken to failure. The webs of the flexure ring were shorn. Even after the webs were broken, however, the hub remained clamped in the housing, and the ring itself remained intact. The ring and the components attached to it (the adapter, floating head, and counterweight) were constrained within the housing and were not able to come out. This was important, because it showed that even if the flexure ring were to fail during the flight test, that the sensor would not come apart and pose a risk to any other part of the vehicle. Figure 68 shows the broken ring being constrained within "Housing b" after the gage was disassembled for inspection. Even more importantly, this failure mode can be avoided. When a sleeve with the same diameter as that of the engine wall mounting hole was fitted over the floating element, the floating head would strike the sleeve at high loads, but would not fail since the deflections were constrained. Once the floating head was constrained in this manner, the gage survived the tests at the aforementioned frequencies and g-loadings without any problem. Visual inspection of the flexure ring after these second tests did not reveal any distortion of the webs. For the actual flight test, the mounting hole in the engine will act as a mechanical stop in the same manner, and prevent failure of the flexure ring due to vibrational loads.

Since the survivability tests were done with non-instrumented flexure rings, no data was recorded from the skin friction gage during those tests. Once the dummy gage survived the shaking tests at high levels, a gage with an instrumented flexure ring was shaken. The purpose was to determine the frequency response of the skin friction sensor. These tests were done at lower g-loadings of about $1 \mathrm{~g}_{\mathrm{rms}}$. This allowed the frequency 


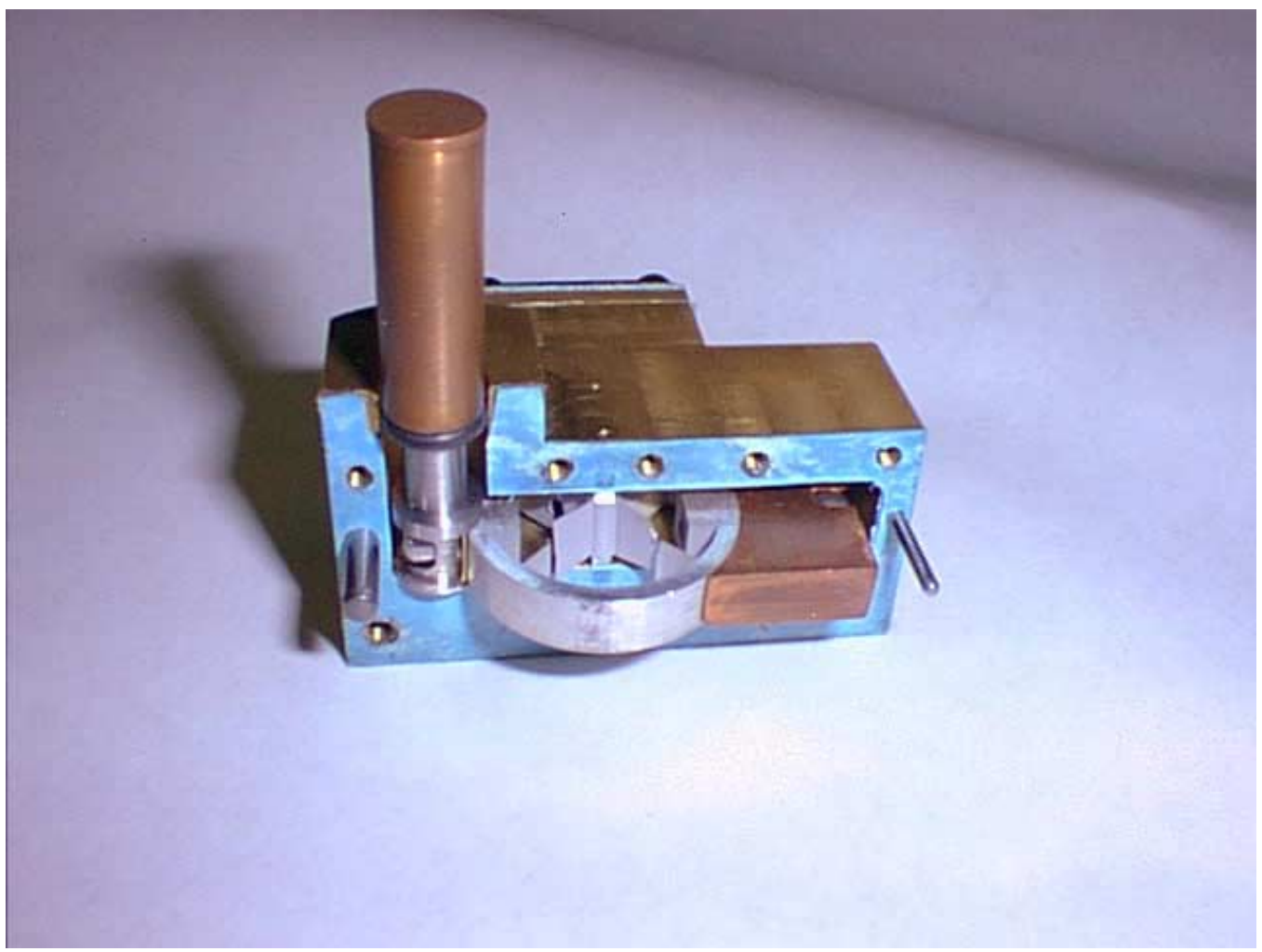

Figure 68: Inspection of Broken Flexure Ring Constrained Within Housing

response of an instrumented flexure ring to be determined without exposing it to the higher load levels.

For these tests, the skin friction sensor was mounted to the shaker, and the DSA recorded the output from the skin friction sensor. The source signal that was sent to the shaker was a burst-chirp sine wave sweep from 15 to $3215 \mathrm{~Hz}$. The DSA produced frequency response function charts during these tests, where the input $(\mathrm{F})$ was taken to be the source signal that was sent to the shaker, and the output $(\mathrm{X})$ was taken to be the signal from the skin friction gage. This was done for all three geometric axes of the sensor.

Figures 69 and 70 show the frequency response function magnitude and phase results, respectively, for the $\mathrm{x}$-axis tests. Figure 71 shows the coherence from these tests. The $\mathrm{x}$-axis corresponds to the axial direction of the flight vehicle and the direction of the design loading on the skin friction sensor. This is the most important of the axes, since the vibration was in the direction of motion of the floating element. Figure 69 shows the first mode of vibration to be at about $40 \mathrm{~Hz}$, with a larger second peak at about $400 \mathrm{~Hz}$. The phase, shown in Figure 70, was good, with very little lag except at the natural frequencies and at frequencies above $2000 \mathrm{~Hz}$. 
The coherence, shown in Figure 71, was not as good as might be expected. A good coherence would display a value near 1.0 at all frequencies. The coherence obtained from these tests, however, was somewhat less than one. The coherence was even worse for the $y$ - and z-axes, especially at lower frequencies. Attempts were made to improve the coherence by using different window functions on the DSA, but this did not

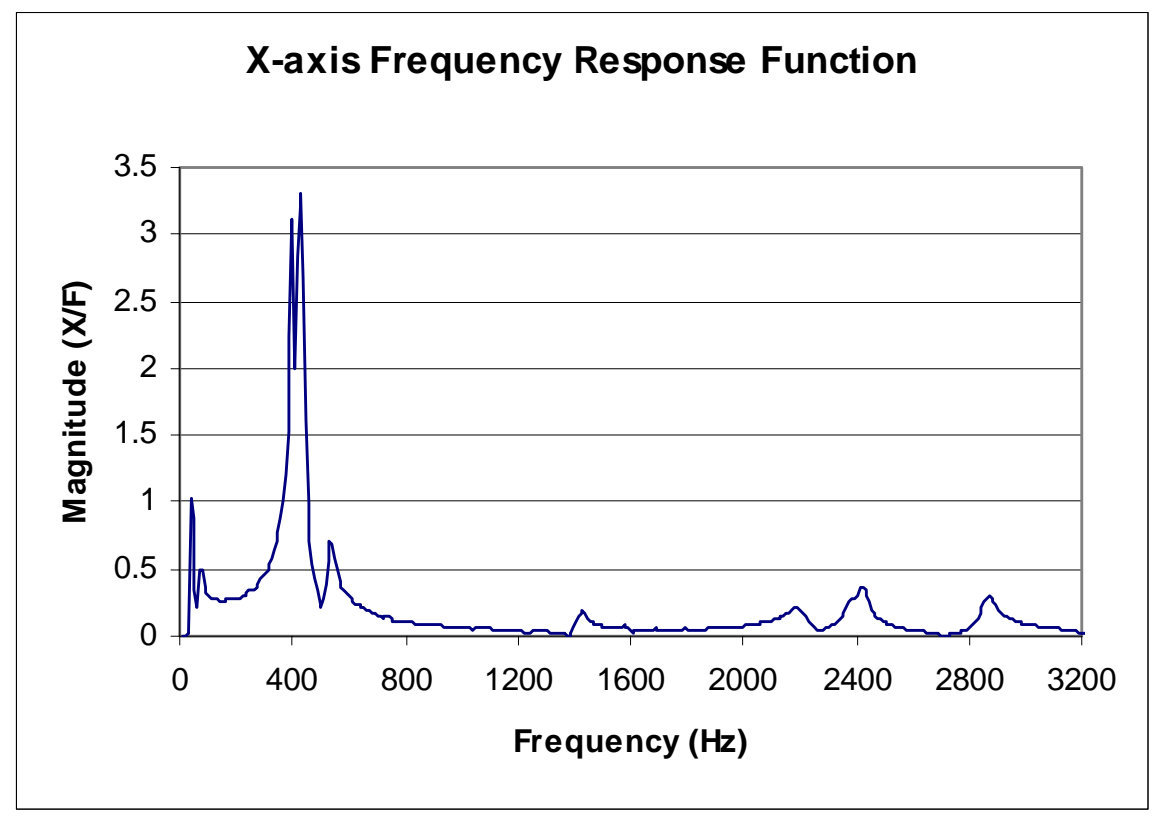

Figure 69: X-Axis FRF Magnitude

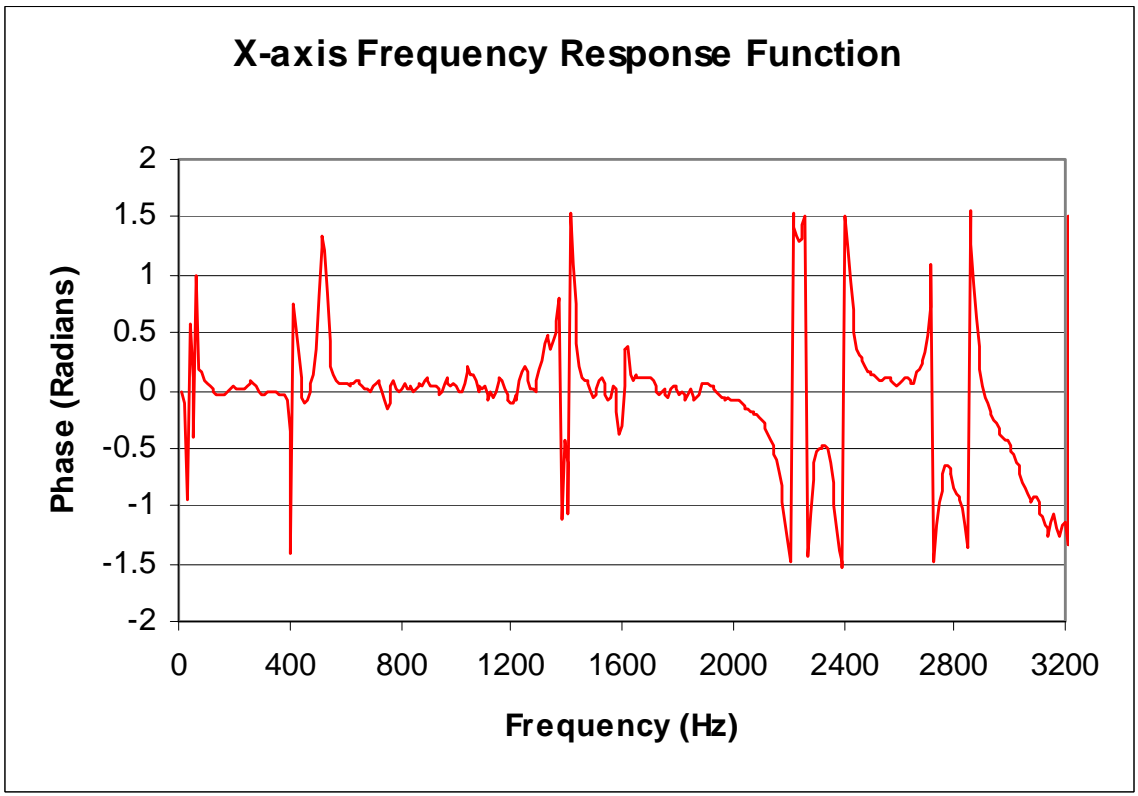

Figure 70: X-Axis FRF Phase 


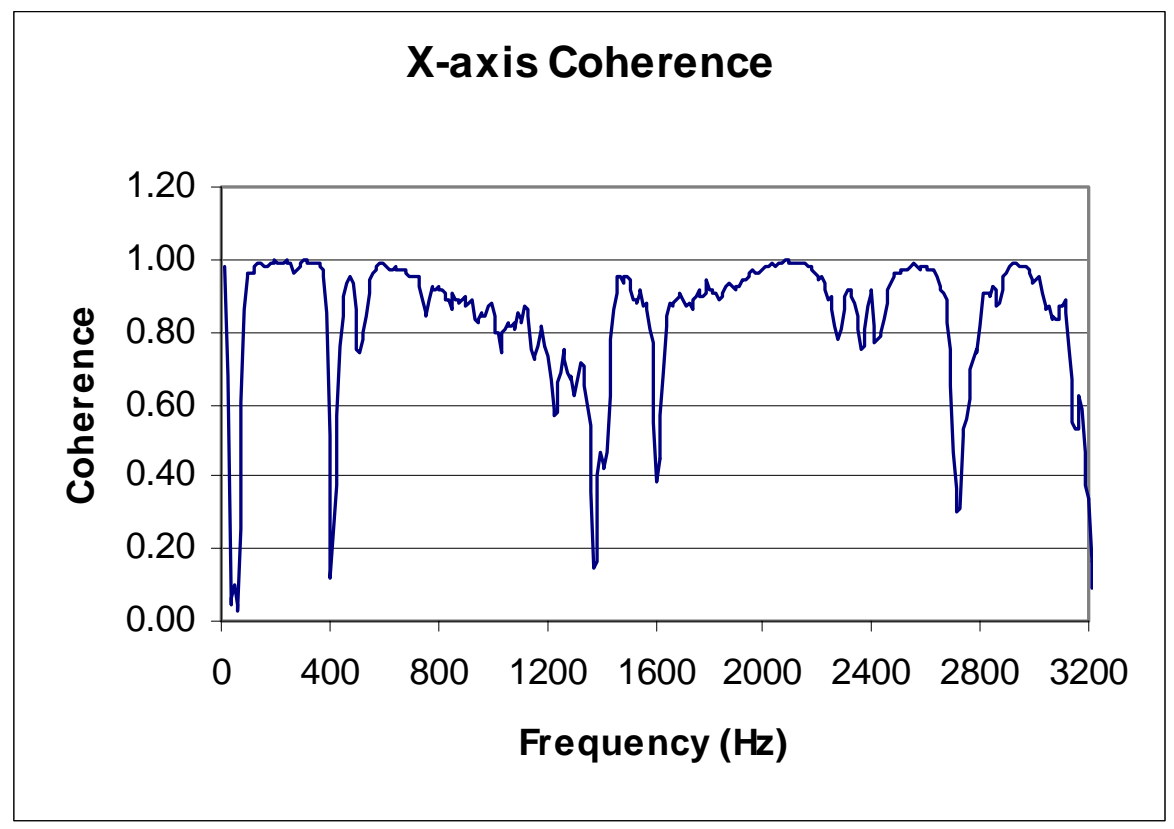

Figure 71: X-Axis Coherence

prove helpful.

There is a possible explanation for the less-than-ideal coherence. The dominant motion of the flexure ring is the rotation about the hub, which corresponds to a motion of the floating element in the x-axis. So at low frequencies especially, the dominant response of the skin friction gage is likely to be in its $\mathrm{x}$-axis direction, even when the forcing vibration is in a different direction. This might explain the poor coherence for the $y$ - and z-axes at low frequencies. The fact that the frequency response functions for all three axes look similar at low frequencies supports this. For the $\mathrm{x}$-axis, imperfect alignment between the shaker and the skin friction sensor may be to blame. The sensor was mounted onto the shaker by means of a bracket. The bracket had threaded holes by which the skin friction sensor was fastened to it. In the $\mathrm{x}$-axis orientation, these holes were not perfectly aligned, so that the skin friction sensor was skewed by a few degrees. This meant that while the shaker was moving vertically up and down, the actual x-axis of the skin friction sensor was a few degrees off of vertical. The transfer function for the shaker-bracket-gage system was unknown. This could explain why the output was not well correlated to the forcing function, leading to the lower coherence.

After the vibration tests were done the skin friction gage was calibrated with the static calibration method. It was important to know whether or not the shaking had in 
any way altered the calibration or sensitivity of the sensor. It was found that the calibration of the sensor after the shaking tests was identical to the calibration done beforehand. The shaking had not affected the sensitivity of the gage.

\subsection{Pressure Tests}

The skin friction gage was required to pass environmental tests for qualification for the flight test. These included pressure and temperature tests. These tests were done to determine the response of the sensor to the temperatures and pressures expected during the flight test.

The pressure test was performed at NASA Dryden Flight Research Center. The skin friction gage was placed in a pressure chamber. The wires for the gage were routed through a pass-through in the wall of the chamber to the 2310 signal conditioning amplifier and thermocouple multimeters so that data could be recorded during the tests. A mass of 2 grams was hung from the floating head of the skin friction sensor to simulate a load. The chamber was then sealed and the pressure was reduced to correspond to different altitudes. Each altitude level was held for about 10 minutes before continuing to the next data point. It was planned to simulate up to $120,000 \mathrm{ft}$, but the pressure chamber was not able to go any further than $96,000 \mathrm{ft}$. The temperature in the chamber remained at ambient during the pressure tests.

Figure 72 shows the results of the pressure test. As can be seen, there was virtually no change in the output of the skin friction sensor during the test. This was expected since the internal volume of the skin friction sensor was not pressurized or sealed and there was no oil filling the interior space. Calibration of the sensor after the pressure tests indicated that the sensitivity of the sensor had not changed. This proved that the gage was not sensitive to pressure and could survive the altitude expected during the flight test without difficulty. 


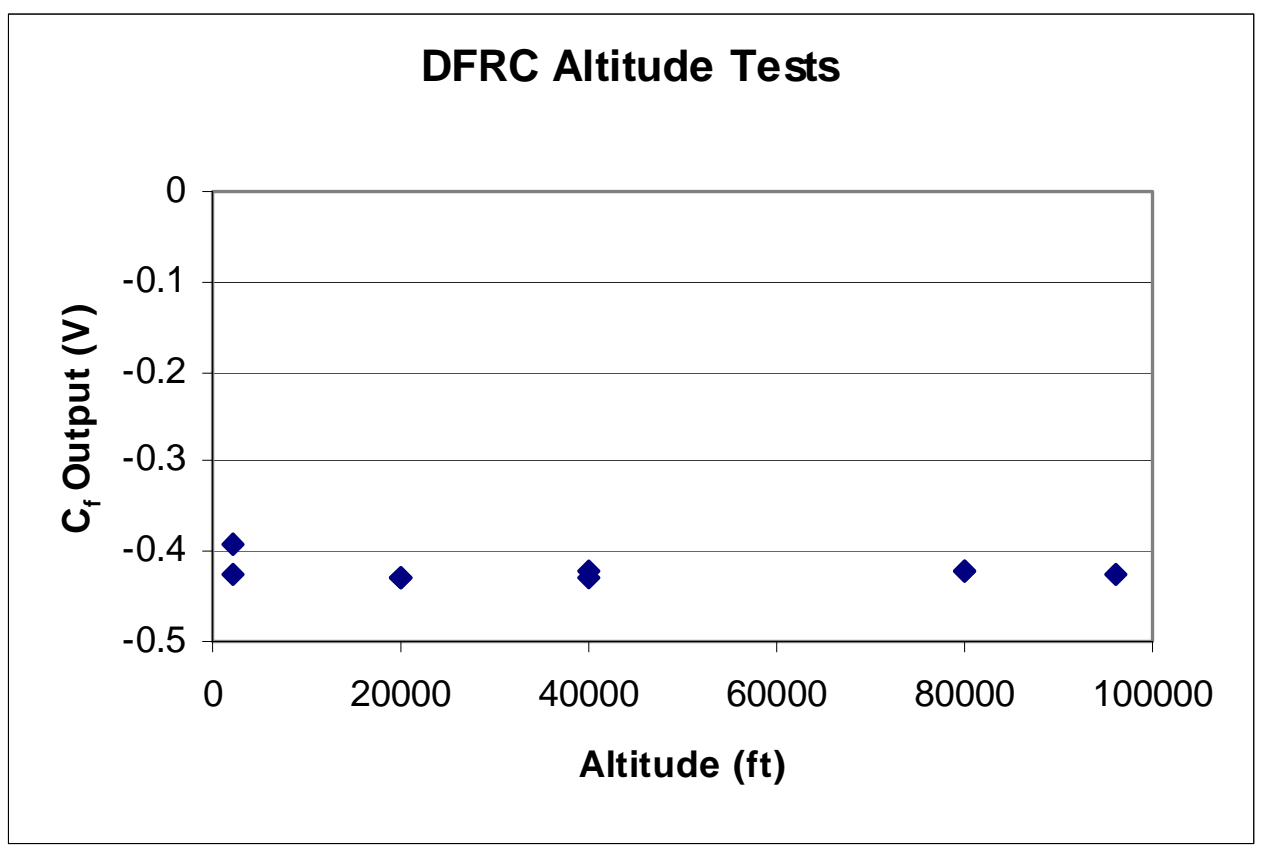

Figure 72: Pressure Environmental Qualification Test Results

\subsection{Temperature Tests}

The skin friction gages were also required to pass temperature environmental qualification tests. These tests were to examine the ability of the gages to function at the temperatures expected during the flight test. Those temperatures range from a low of $40^{\circ} \mathrm{F}\left(-40^{\circ} \mathrm{C}\right)$, which corresponds to the temperature at the altitude of the flight test, to a high of $160^{\circ} \mathrm{F}\left(71^{\circ} \mathrm{C}\right)$, which can occur when the vehicle is sitting on the tarmac before takeoff (NASA Dryden, where the flight is to take place, is located in the Mojave Desert in California).

The NASA Dryden pressure chamber was also used to do transient temperature tests. The skin friction gage was placed in the chamber at ambient pressure, and the sensor output was balanced. Then a 2-gram weight was hung from the head of the gage in the same manner as during calibration. The temperature of the chamber was then reduced to $-40^{\circ} \mathrm{F}$. The temperature of the skin friction gage was monitored using the thermocouple built into the housing. The output from the Wheatstone bridge was also monitored. The temperature of the skin friction gage fell slower than the air in the chamber, due to the thermal mass of the sensor. As the temperature of the gage fell, the temperature and output of the gage were recorded. 


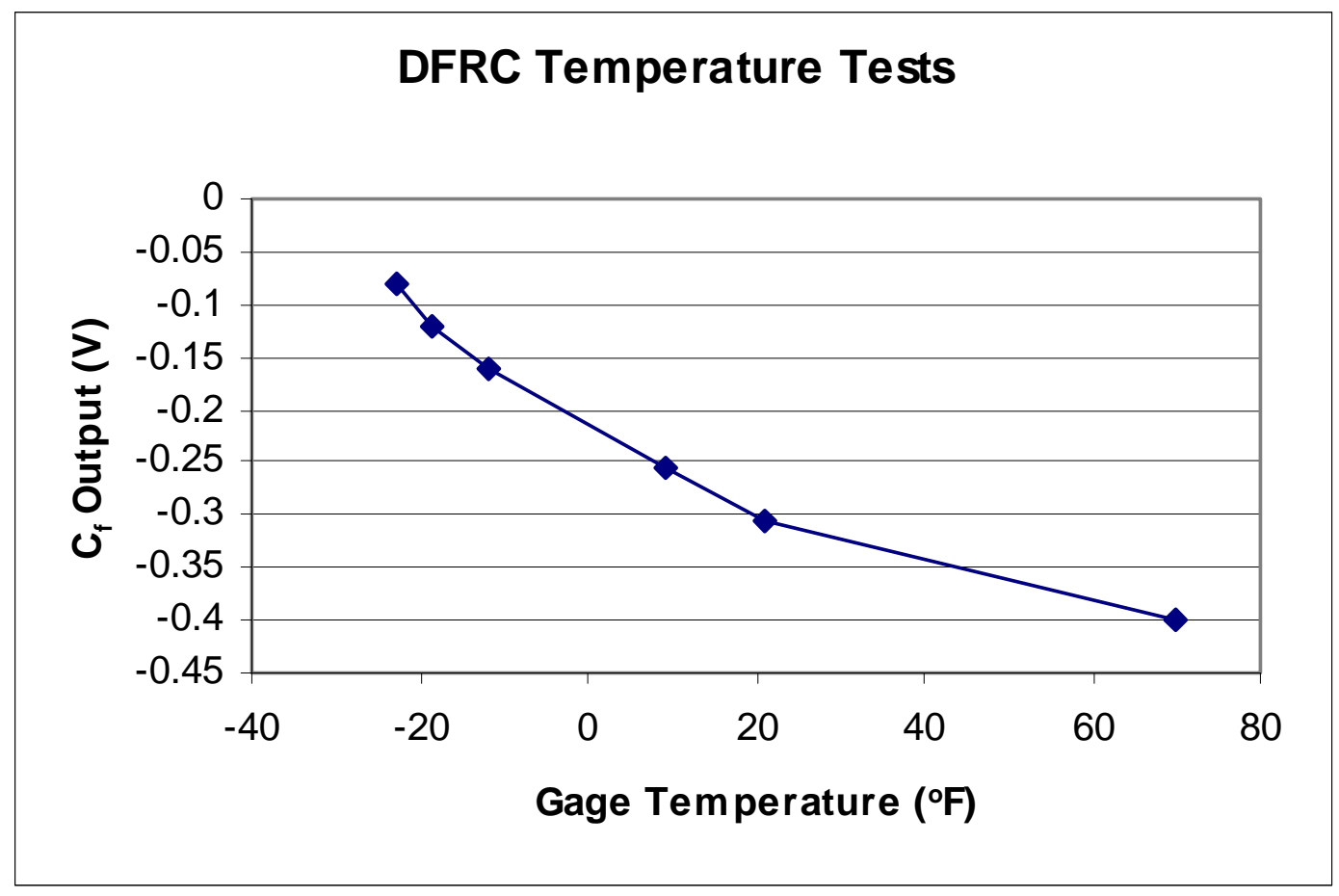

Figure 73: Transient Temperature Test

The result of this test is shown in Figure 73. At ambient temperature the output was -0.4 volts, which corresponds to the 2 gram mass applied to the floating element. As the gage temperature fell, the output steadily increased in a nearly linear manner. The temperature of the skin friction gage was not allowed to reach a steady state, however. The output of the gage may have continued to change even after the temperature had reached a constant level. While the skin friction gage was still very cold, the chamber was opened, and the weight was removed from the floating element. The output of the gage increased from -0.08 volts to +0.32 volts, a change of +0.4 volts. This change in voltage due to the mass was the same as the calibration done at ambient conditions. This indicated that although the output had drifted due to temperature, the sensitivity had remained the same.

Steady state temperature tests were also attempted. These were done in the Virginia Tech Engineering Science and Mechanics Department using a Blue M Electric ETC-04D-C Environmental Test Chamber with a Pro-550 Controller. This test chamber has a two-stage cascade refrigeration system and is capable of testing at temperatures from $-80^{\circ} \mathrm{C}$ to $+190^{\circ} \mathrm{C}$. The gage was placed in the chamber with wires routed through a pass-through to the 2310 signal conditioning amplifier and an Omega thermocouple DC 
millivolt amplifier. Output from the 2310 and the thermocouple amplifier were read on multimeters.

For each test point the test chamber was programmed to reach and hold a steady temperature. The gage was then allowed to reach the temperature of the chamber and remain there for about 45 minutes before moving to the next test point. When the temperature was changed the output from the gage would go through a transient response before approaching a steady value. Both the maximum transient value and the steady value were recorded for each test point.

The result of these tests is shown in Figure 74. The circles represent the maximum transient value, and the diamonds represent the steady value reached. The transient output was always greater than the steady value. Like the tests in the Dryden test chamber, the output increased with decreasing temperature and decreased with increasing temperature. The output did not continue to increase at the coldest temperature. Similarly, the output did not continue to decrease at the hottest temperature. After the hottest temperature test point, the chamber was returned to ambient temperature. The gage output, however, did not return to zero during the following few hours.

In order to study this more thoroughly, the hottest data point $\left(160^{\circ} \mathrm{F}\right)$ was repeated

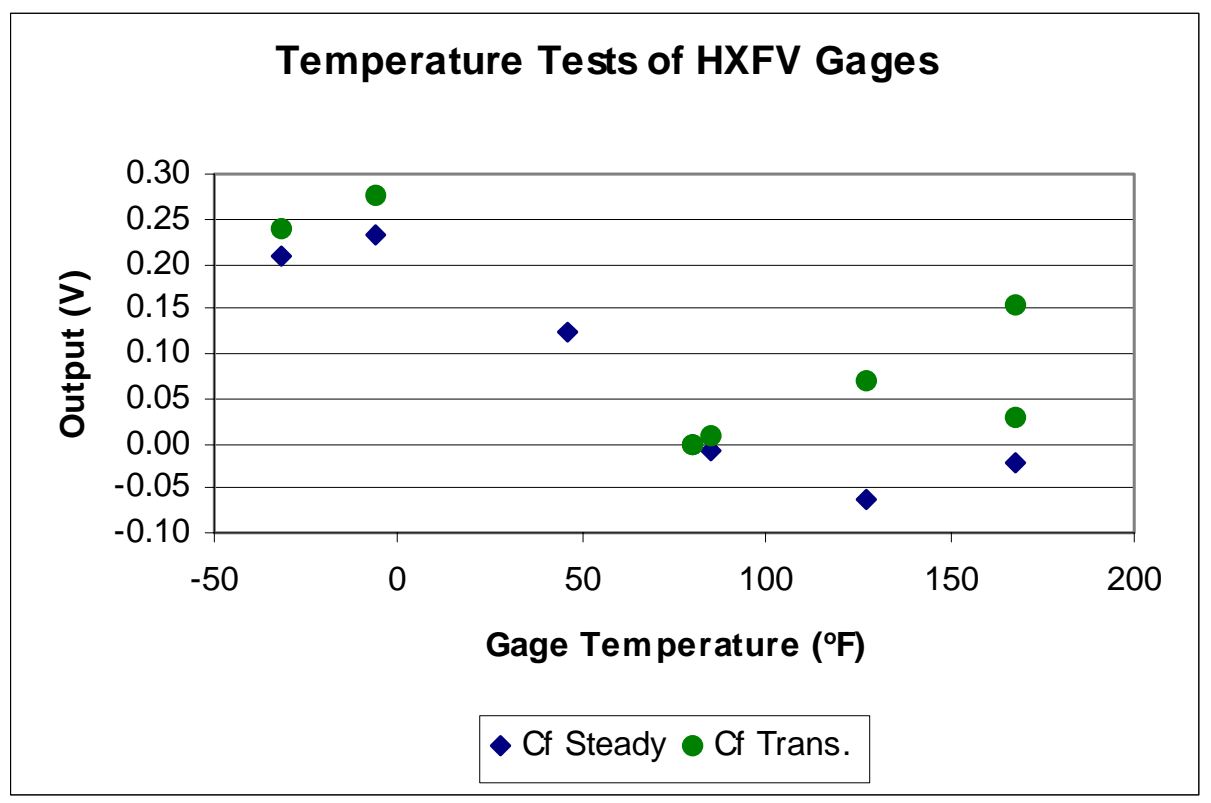

Figure 74: Steady State Temperature Test 
over a much longer time period. The chamber and gage were allowed to remain at the test temperature for well over an hour. The chamber was then opened to the atmosphere. The gage was allowed to slowly reach ambient temperature overnight. The temperature and output from the gage were recorded during the following 22 hours.

Figure 75 shows the result of this test. The data points were recorded chronologically in the clockwise direction, starting from the ambient temperature. The data points on the upper portion of the chart that are connected by the line were recorded before the test chamber was opened to the atmosphere. The data points after the break in the line were recorded as the gage cooled after the test chamber was opened. The upper portion of the graph shows the initial rise in the output during the transient response, and then the approach to a steady level of about -0.1 volts. Once the chamber was opened, the voltage fell a small amount as the gage began to cool. It was at this point that the gage was left overnight. When the measurements were resumed in the morning, the temperature had returned to ambient, and the output also had approached zero.

Once again, the gage was calibrated after the temperature tests. These calibrations showed that the exposure to the hot and cold temperatures did not alter the calibration of the sensor. Visual inspection showed no damage or change in the gage.

These temperature tests were of limited use. The actual flight temperature

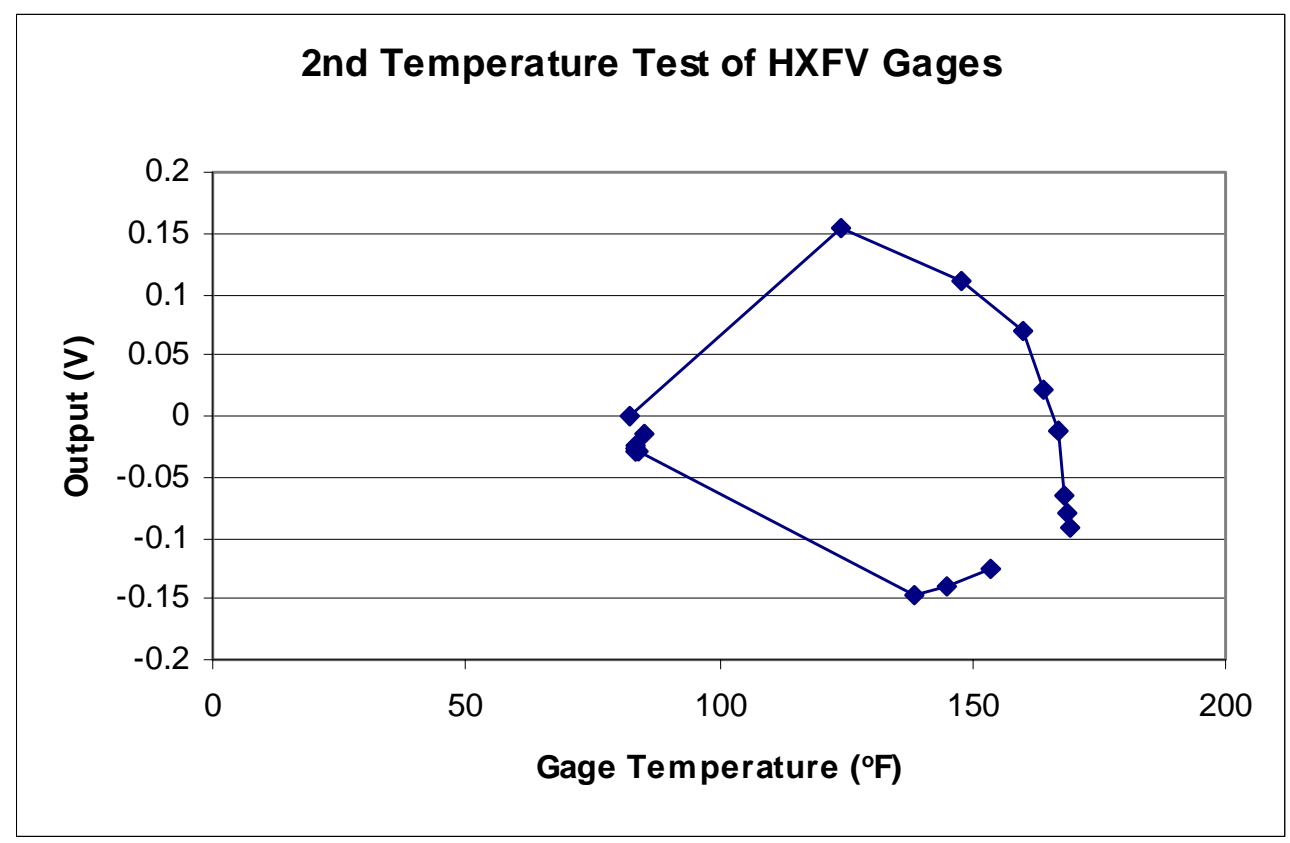

Figure 75: Single Temperature Response Profile 
environment is a fast changing transient and very difficult to reproduce in a lab, and the steady-state response of the gage does not help much in predicting the transient response. Also, since the gage is to be mounted in the engine of the flight vehicle, the temperatures that it sees may be well above the $160^{\circ} \mathrm{F}$ that was specified for these environmental tests. Therefore, the behavior of the skin friction gage in the flight may or may not be like the behavior in these tests. The most important fact that was determined from these tests was that the output of the gage did change with temperature, but the sensitivity did not.

\subsection{Heat Load Tests}

It was desired to test the skin friction gage in a heating environment closer to that expected in the scramjet engine during flight. To do this it was necessary to reproduce the wall heat flux that was estimated for the engine near the location of the sensors. Two facilities were used to attain this goal. Both facilities used radiative heat flux sources. The heat to the engine wall during the flight test, however, will be transferred via convection. Methods for accurately reproducing high levels of convective heat fluxes are not available. The best way to accurately simulate high heating loads, then, is to do so with calibrated radiative systems.

\subsubsection{NASA Dryden Tests}

The first set of heat load tests was performed at NASA Dryden Flight Research Center in the Flight Loads Laboratory. This laboratory is equipped with a heat flux gage calibration system that can reproduce high heat flux levels. The equipment includes a Thermogage Model $48 \mathrm{KW}$ Furnace and a flat plate heater assembly. The furnace provides a high power voltage across a thin, flat graphite plate. The current flowing through the flat plate causes it to heat to extreme temperatures. At these extreme temperatures, the plate glows and radiates heat flux values up to $400 \mathrm{Btu} / \mathrm{ft}^{2}-\mathrm{s}$. A calibrated Gardon-type heat flux gage is placed on one side of the flat plate to monitor the heat flux level. The test article is placed on the opposite side and at the same distance from the flat plate in order to be exposed to the same heat flux.

For these tests a special mounting bracket was constructed for the skin friction gage. This consisted of a one-inch thick copper block to simulate the surrounding engine wall. The holes for the skin friction gage floating element and \#1/4-20 mounting screws 
were machined into this block so that the floating element would be flush with the surface. The flat plate heater was oriented vertically, so the copper block was designed to be vertically mounted such that the surface would be parallel to the flat plate. An aluminum plate was fastened to the copper block so that the entire fixture could be clamped in place. Figure 76 presents a sketch of the skin friction gage mounted in the copper block mounting assembly opposite the flat plate heater. Only the electrodes and the graphite flat plate itself are shown in the sketch for simplicity. When mounted in this configuration the surface of the floating element and the copper plate are exposed to the heat flux in the same manner as would be in the Hyper-X engine.

To simulate the heating environment of the flight vehicle it was desired to expose the gage to a step increase in heat flux, hold that level for several seconds, and then return to zero heat flux. This would reproduce the opening of the engine cowl at Mach 10 and the firing of the engine for several seconds before the cowl is closed again. There is no flow through the engine, and therefore no heat flux, before and after the cowl is opened and closed. However, this proved difficult to reproduce in the Dryden Flight Loads Laboratory. The flat plate heater ramps up to the test heat flux level over about 25

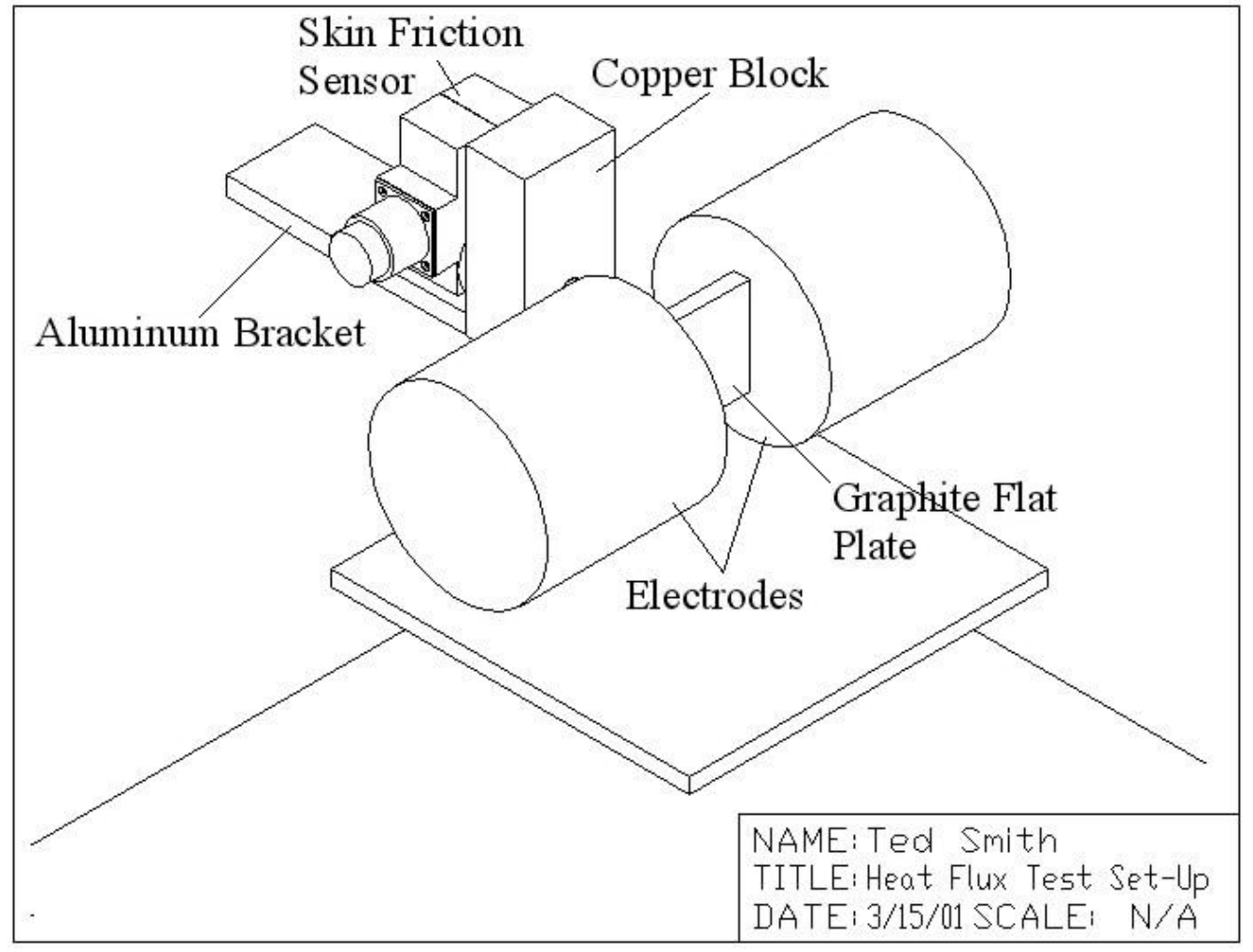

Figure 76: Heat Load Test Setup Sketch 
seconds. This length of time is about the same as the total engine-on time of the flight test. A shutter system was designed to raise and lower a graphite plate from between the heater plate and the copper block. It was hoped that this could be used to shield the gage during the ramp-up and ramp-down periods. Unfortunately, the high temperatures proved to be too much for the shutter system, and the idea had to be abandoned.

A second difficulty also prevented the Dryden tests from providing useful information. The flat plate heater was easily capable of radiating the heat flux levels that were predicted for the flight test. These levels were between 70 and $130 \mathrm{Btu} / \mathrm{ft}^{2} \mathrm{~s}$. But the surface of the copper block and the floating element are machined copper, which has an emissivity of between 0.07 and 0.15 . This meant that the copper block and floating head were absorbing only roughly $10 \%$ of the radiated heat flux.

Because of the emissivity problem, there was considerable uncertainty in the amount of heat flux that was absorbed by the sensor. It also meant that the absorbed heat flux was much less than the predicted level for the flight test. And the lack of a shuttering system led to exposure of the sensor to the heat flux for periods much longer than the expected test time for the flight. These problems with the tests at NASA Dryden led to a second set of tests.

\subsubsection{Vatell Tests}

The second set of tests was performed at Vatell Corporation in Christiansburg, Virginia. Vatell manufactures heat flux sensors and also performs heat flux tests and calibrations. A flat plate heater facility very much like the one at NASA Dryden was used at Vatell to reproduce the predicted heat flux of the Hyper-X flight test.

An added capability at Vatell was the ability to traverse the test article on rails toward and away from the flat plate heater during testing. This allowed the skin friction gage to be pulled away from the heater plate and shielded during the ramp-up and shutdown periods. In this way the skin friction gage could be exposed to the heat flux only for the brief amount of time expected during the flight test.

It was also required that the skin friction gage should absorb the entire amount of radiated heat flux. To ensure this, the surface of the gage and the copper block were coated with soot. Figure 77 shows the skin friction gage mounted in the copper block and coated with soot prior to testing. The emissivity of the surface of the copper block 


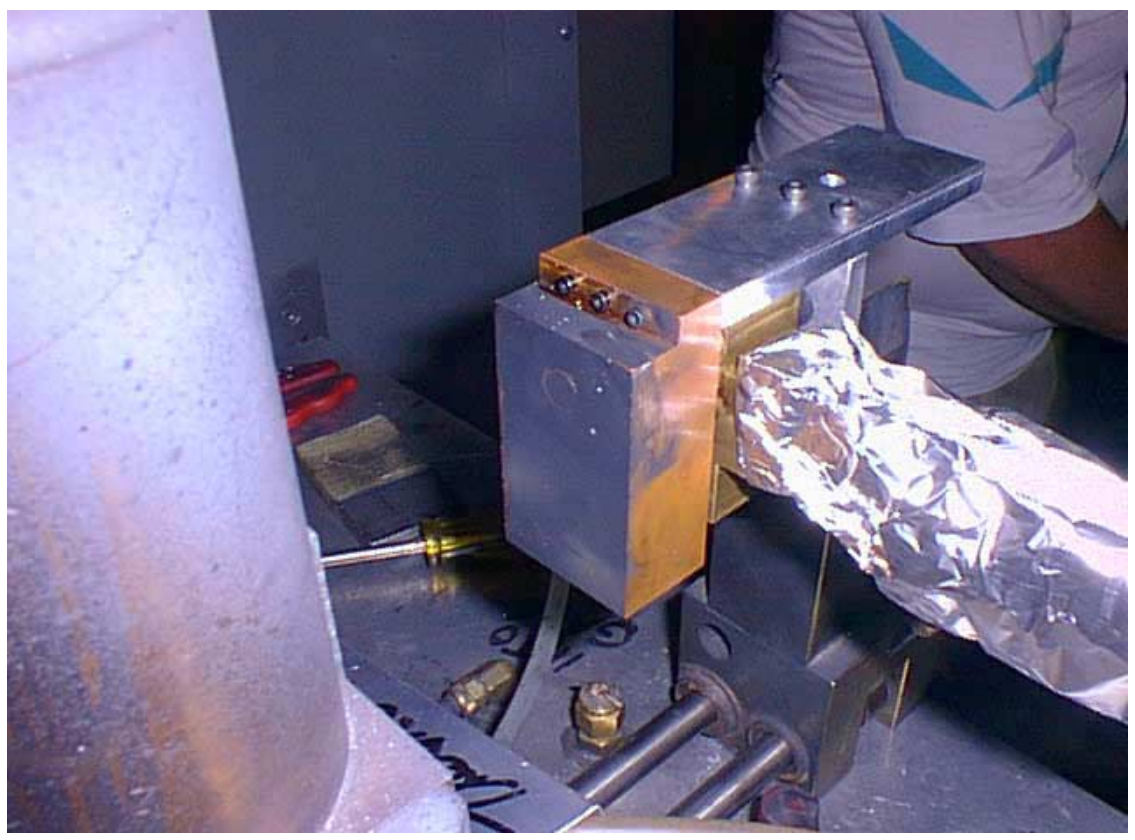

Figure 77: Heat Load Test Setup: Skin Friction Gage Mounted in Copper Block and Coated with Soot

and floating element when coated with the black soot was very near 1.0. This meant that during the heat load tests at Vatell the skin friction sensor absorbed almost the same amount of heat flux as was radiated by the flat plate.

The skin friction sensor was tested at 30 and $70 \mathrm{Btu} / \mathrm{ft}^{2} \mathrm{~s}$. Before each run the output from the skin friction sensor was balanced to zero. The output from the Wheatstone bridge and the Type $\mathrm{K}$ thermocouple in the housing were recorded on a PC via a National Instruments A/D board using LabView data acquisition software. While the heater plate was ramped up to the test point the skin friction gage and copper mounting block were covered with a tin sheet and traversed on rails away from plate. Once the flat plate reached the designated heat flux, the shielding was removed and the gage was traversed up to the flat plate for several seconds. Then the gage was traversed away from the plate again and the power to the heater plate was cut. Figure 78 shows the skin friction gage withdrawn from the heater plate and traversed up to the flat plate. Figure 79 shows the gage withdrawn from the plate and shielded as the flat plate heats up. 


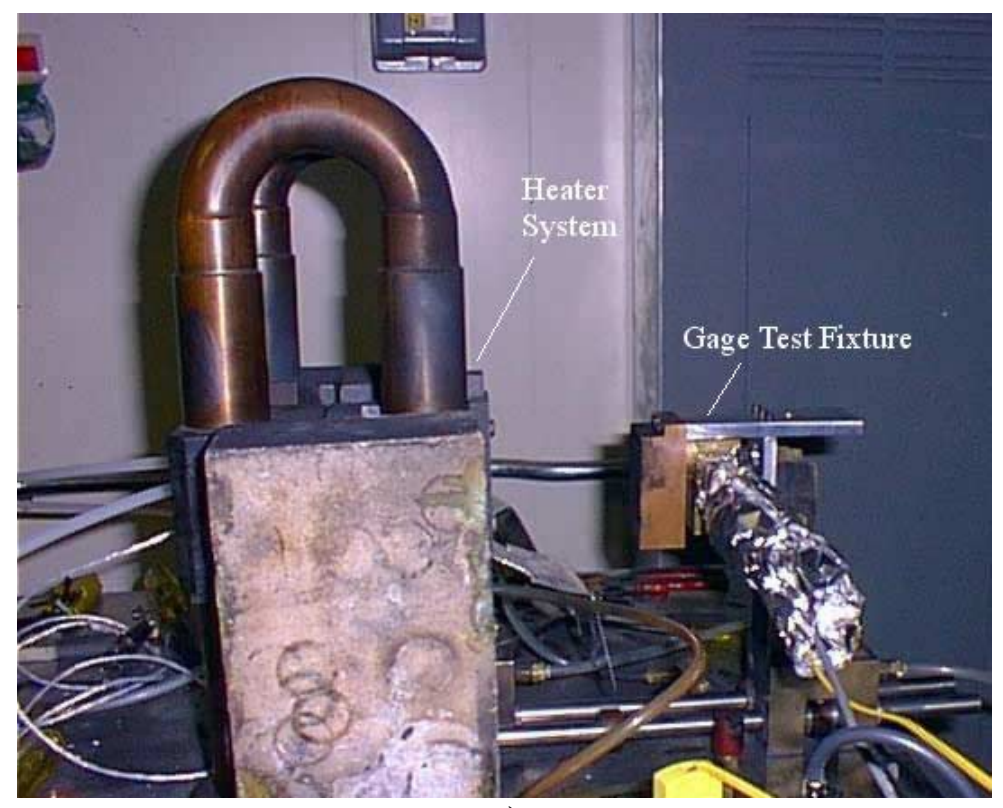

a)

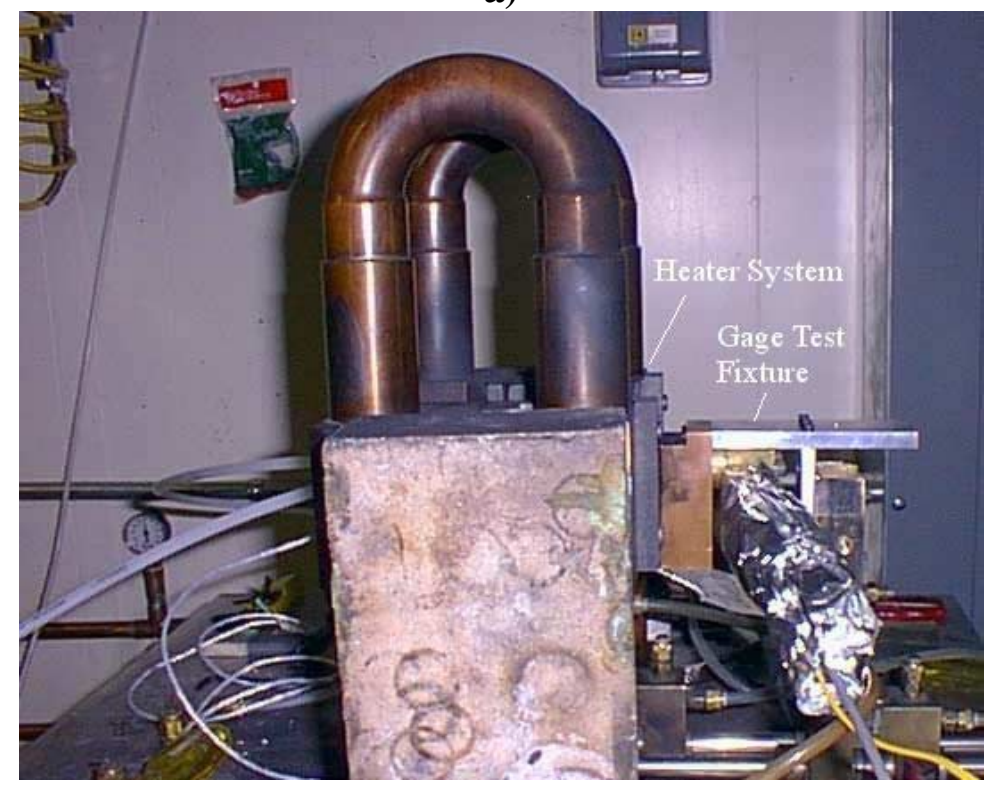

b)

Figure 78: Heat Load Test Setup: a) Gage Withdrawn b) Gage Up Close to Heater Plate 


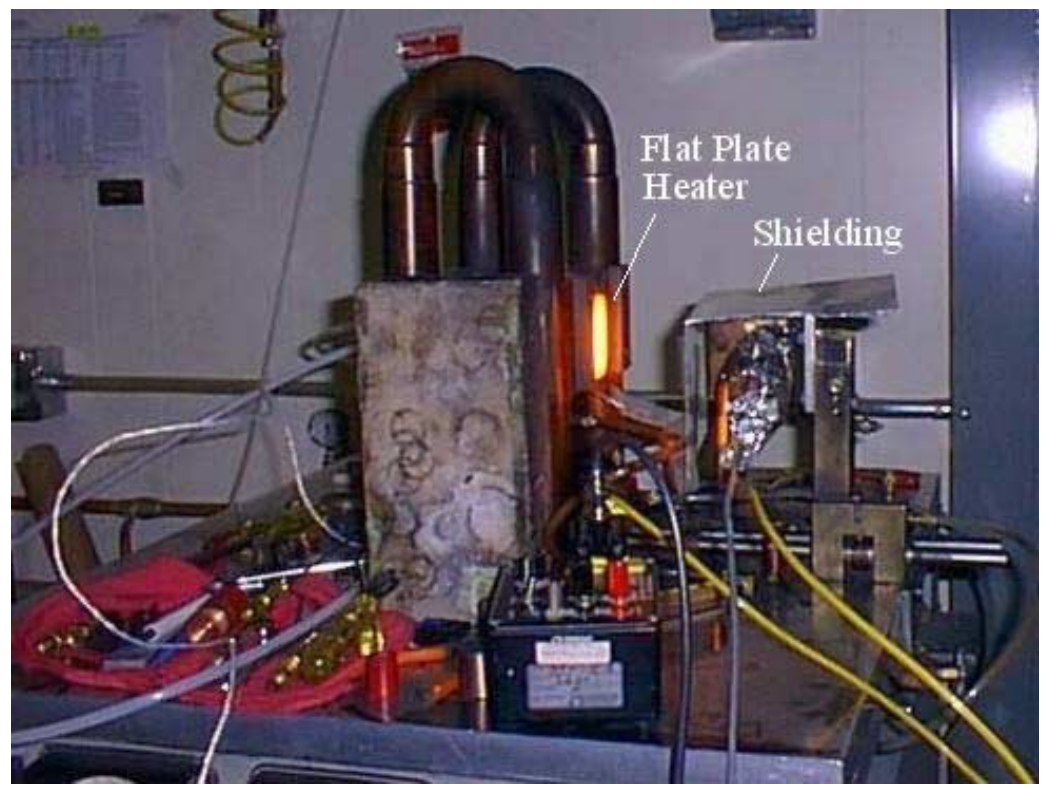

Figure 79: Heat Load Test Setup: Shielded Gage During Heater Ramp-Up

Results of the first of these tests are shown in Figure 80. Here the heat flux level was $30 \mathrm{Btu} / \mathrm{ft}^{2} \mathrm{~s}$. Figure 80 shows the temperature and Wheatstone bridge output from the skin friction gage over time. The temperature was measured by the thermocouple mounted in the housing. The large spikes in the bridge output are due to the test fixture hitting the stops of the guide rails as the fixture was traversed towards and away from the flat plate. These impulsive loads effectively mark the beginning and end of the heat flux exposure period. Several seconds of data were recorded before and after the exposure period. Before the exposure the output from the sensor was steady near zero and the temperature was steady at room temperature as seen in the chart. At about eight seconds into the run, the shielding was removed and the gage was moved within range of the flat plate heater. The gage was in the test position until about 15 seconds into the run, for a total exposure time of about seven seconds. Then, the heater was powered down and the gage was withdrawn from the plate. The temperature and bridge output remained nearly unchanged during the first several seconds of the test time. Then, after several seconds, the temperature and the output from the skin friction sensor began to rise, the temperature rising from about $80^{\circ} \mathrm{F}\left(27^{\circ} \mathrm{C}\right)$ to about $90^{\circ} \mathrm{F}\left(32^{\circ} \mathrm{C}\right)$ and the gage output approaching a maximum offset of about $45 \mathrm{mV}$. The major portion of the temperature and output rise occurred after the exposure time and both seemed to follow the same basic trend. 


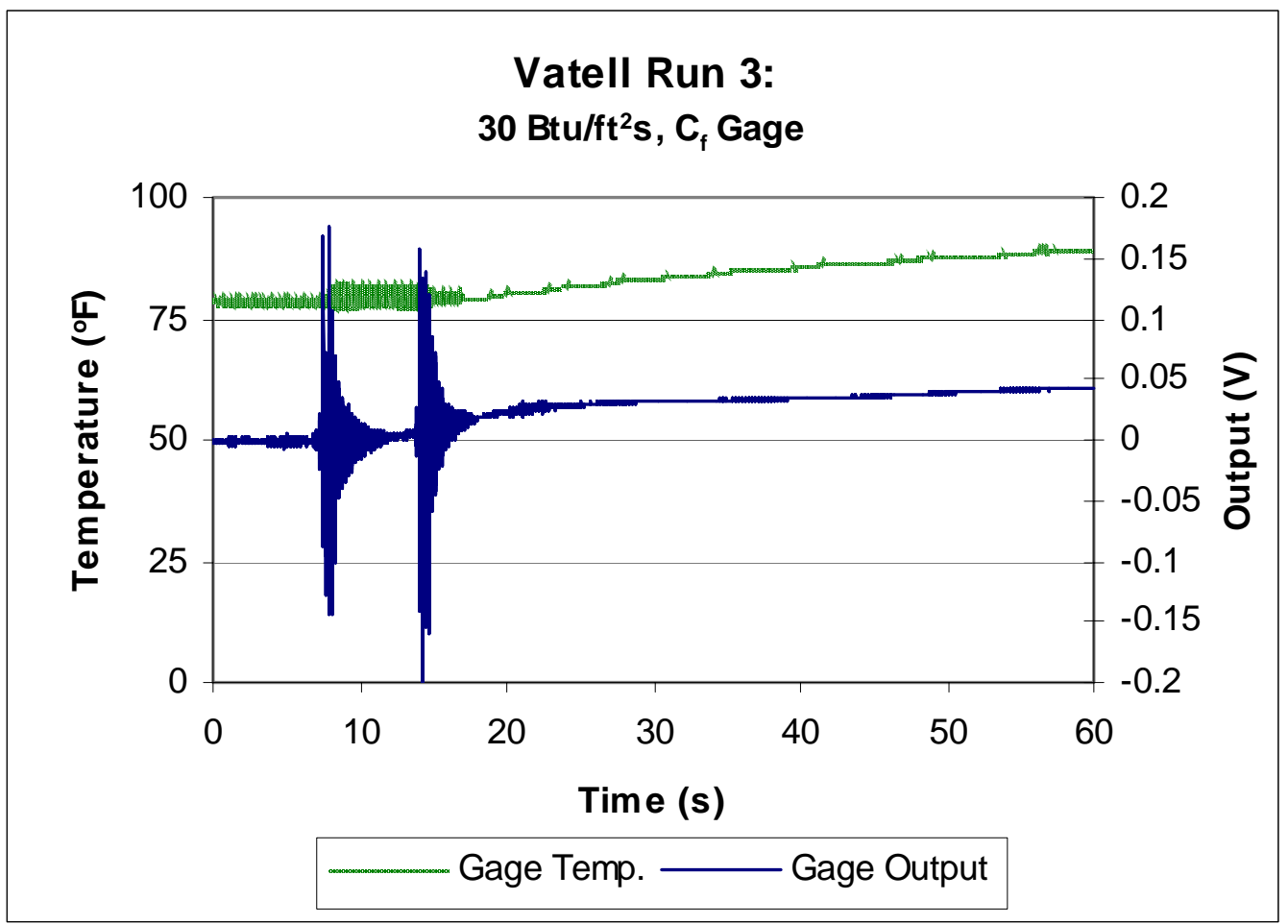

Figure 80: Heat Load Test Results: $30 \mathrm{Btu} / \mathrm{ft}^{2} \mathrm{~s}$

Results of the second of these tests are shown in Figure 81. For this test the heat flux was $70 \mathrm{Btu} / \mathrm{ft}^{2} \mathrm{~s}$. Again, the temperature measured in the housing of the gage and the output from the strain gages are presented. The heat flux exposure period for this test can be seen from the spikes in the gage output, once again caused by the test fixture hitting the stops of the rail traverse system. The beginning of the exposure period was at about 15 seconds into the run, and the end of the exposure period was at about 22 seconds, for a total test time of about 7 seconds. The strain gage output started out at about $-12 \mathrm{mV}$ and began to rise after about 5 seconds of exposure to the high heat flux. Likewise the temperature began at about $90^{\circ} \mathrm{F}\left(32^{\circ} \mathrm{C}\right)$ and began to increase after several seconds of exposure. Similar to the first test, the major portion of the drift in temperature and strain gage output occurred after the test window. The temperature continued to increase up to about $115^{\circ} \mathrm{F}\left(46^{\circ} \mathrm{C}\right)$ and the Wheatstone bridge continued to drift up to about $110 \mathrm{mV}$. These shifts occurred more rapidly and to greater extremes than the during the lower heat flux test, as expected. The internal temperature and the gage output were monitored for several minutes after the test. The temperature reached a maximum of about $140^{\circ} \mathrm{F}$ $\left(60^{\circ} \mathrm{C}\right)$ after a few minutes, before slowly cooling back down. The gage output reached 


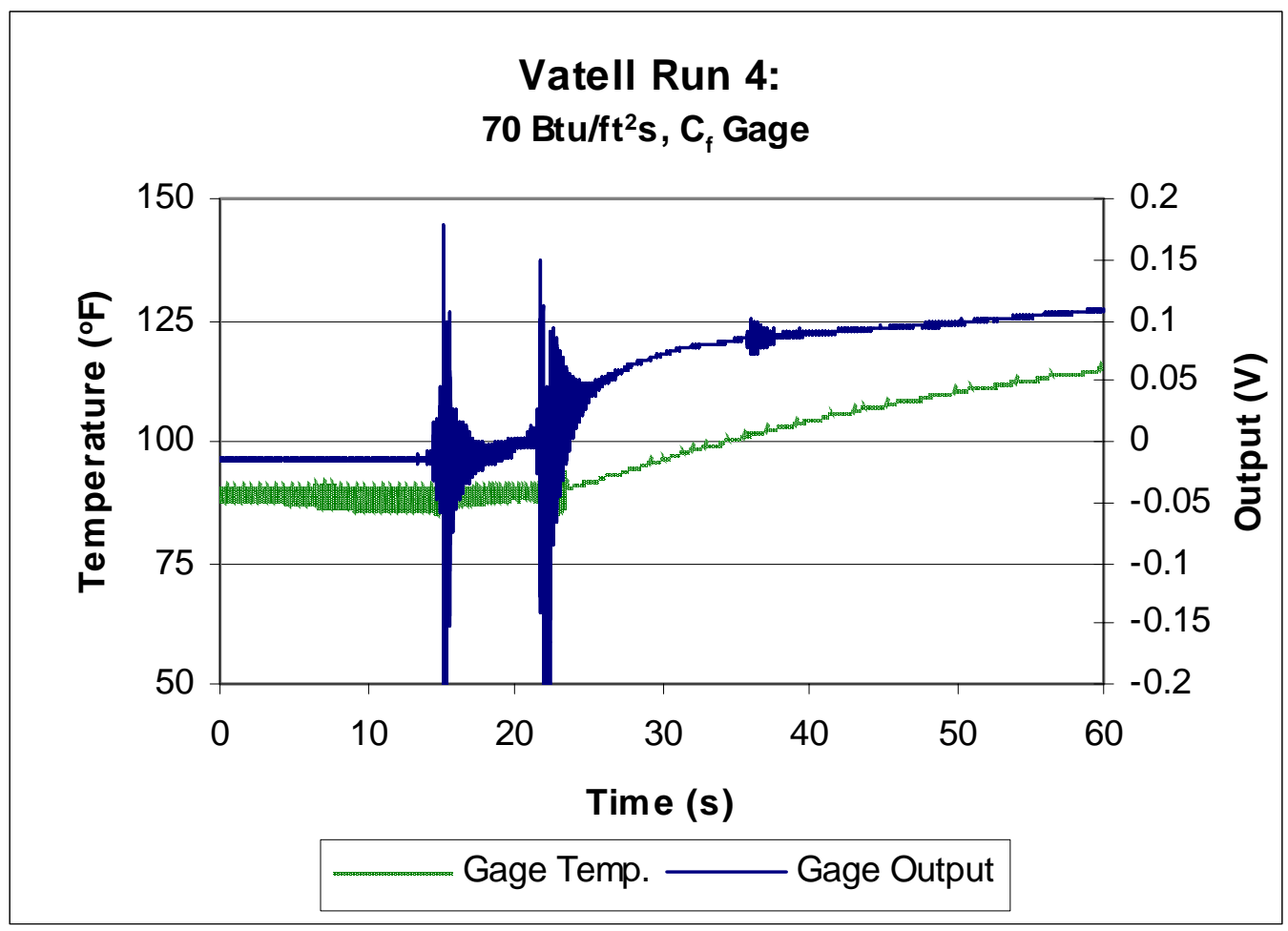

Figure 81: Heat Load Test Results: $70 \mathrm{Btu} / \mathrm{ft}^{2} \mathrm{~s}$

about $150 \mathrm{mV}$ and then slowly returned towards zero.

These tests showed that the skin friction sensor did not give an output directly related to heat flux, but the output did drift due to the temperature increase inside the gage. The increase in temperature and the subsequent drift in the strain gage signal were delayed until several seconds after the initial exposure to the heat flux. Tests of a heat flux gage in the same test fixture (to be discussed later) showed that the temperature of the surface of the copper block reached about $270^{\circ} \mathrm{F}\left(132^{\circ} \mathrm{C}\right)$ during a $70 \mathrm{Btu} / \mathrm{ft}^{2} \mathrm{~s}$ test, and it is assumed that the temperature of the surface of the floating element reached a similar temperature. This means that although the surface of the floating element may get very hot, the sensor has a large enough thermal mass that the thermal wave takes a relatively long time to propagate through the sensor. This was considered to be very good because of the relatively short duration of the engine firing during the flight test. The cowl of the engine will only be open for about 15 seconds during the entire flight, and the engine will only be fired for about 7 of those seconds. Any thermal drift from the skin friction gage during that time period should be minimal and/or easily accounted for in post-processing. This very situation was encountered later during wind tunnel tests. 
After the heat load tests the skin friction gage was recalibrated to determine if the exposure to the heat flux had in any way altered its sensitivity. These calibrations once again showed that the sensitivity remained unchanged. Visual inspection of the sensor showed no damage to the floating element, the gaskets, or any other component of the gage. The gage was not sensitive to the heat flux and was able to survive that hot environment.

\subsection{Cold Flow Tests}

To validate this sensor in a well documented, supersonic flow environment, it was tested in the Virginia Tech 9-inch by 9-inch supersonic wind tunnel. These tests provided the first proof that the design would work in providing accurate measurements of skin friction in a supersonic flow. These tests were conducted at Mach 2.4, with stagnation quantities of $\mathrm{P}_{0}=50 \mathrm{psia}(345 \mathrm{kPa})$ and $\mathrm{T}_{0}=540^{\circ} \mathrm{R}\left(300^{\circ} \mathrm{K}\right)$.

In the tests of the earlier skin friction sensor designs those sensors were mounted directly into the aluminum floor plate of the tunnel test section. Potential problems related to the discontinuity between the aluminum floor plate and the copper floating element were simply ignored. Such a discontinuity in surface material could create a discontinuity in the temperature profile along the tunnel floor, which in turn could lead to discontinuous skin friction values. An improved experiment was designed for this skin friction sensor. It was desired to allow the boundary layer along the tunnel floor to come to an equilibrium state before encountering the skin friction sensor. A copper insert for the tunnel floor plate that would meet this need was manufactured. A general rule of thumb is that the boundary layer will come to equilibrium at a distance of roughly 10 boundary layer thicknesses (108) from a change in the flow. The boundary layer in the supersonic wind tunnel at the test conditions has been measured to be roughly 0.4 inches $(1.016 \mathrm{~cm})$. The copper insert was therefore designed to provide over 4 inches $(10.16$ $\mathrm{cm}$ ) of copper surface upstream of the skin friction sensor location. The copper insert mounted in the aluminum floor plate is shown in Figure 82 before installation in the wind tunnel. A pressure port in the aluminum floor plate adjacent to the sensor was used to obtain a static pressure reading during tests. This was later used in determining the dynamic pressure for non-dimensionalizing the wall shear into the skin friction coefficient. 


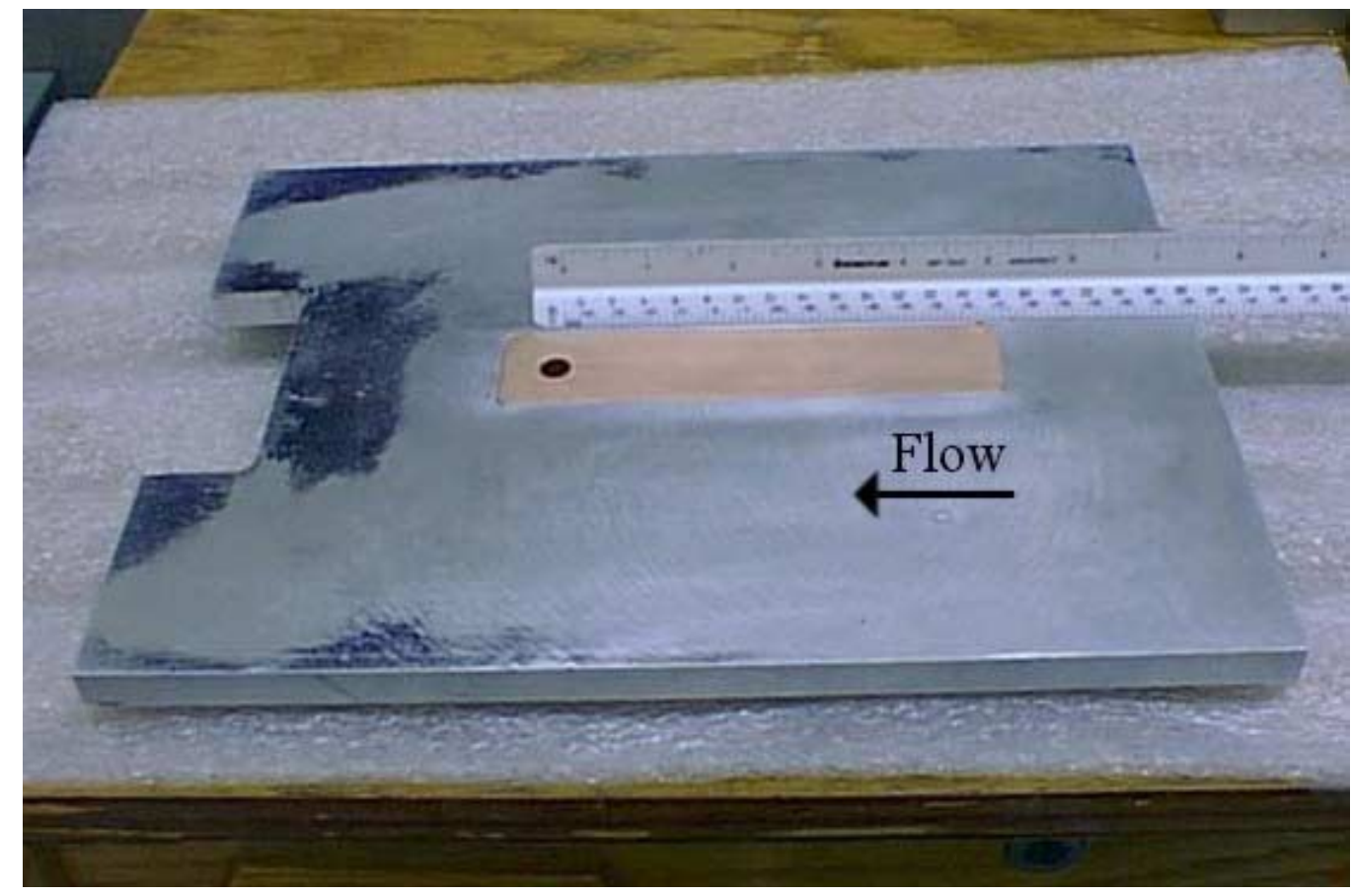

Figure 82: Copper Insert for Supersonic Wind Tunnel Tests

Four tests were conducted in the Virginia Tech supersonic wind tunnel. The skin friction gage performed extremely well in all of the runs, with consistent output. Sample results from two of the runs are shown in Figure 83 and Figure 84. A summary of the results from all of the tests is given in Table 4. Figure 83 shows the shear level from the first run along with the normalized skin friction coefficient result. The shear level decreased slightly during the run, but the skin friction level remained constant. This was because the total pressure (and thus the static pressure) was dropping during the run. The static pressure dropped at the same rate as the shear, so that the skin friction coefficient remained the same. The results from the last run are shown in Figure 84. For this run the tunnel was shut down before the end of the data record. This made it possible to confirm that the output returned to zero after the test. The violent starting and stopping of the

\begin{tabular}{|c|c|c|}
\hline Run & $\tau_{w, \text { avg }}(\mathrm{Pa})$ & $\mathrm{C}_{\mathrm{f}, \mathrm{avg}}$ \\
\hline 0 & 236 & 0.0023 \\
1 & 245 & 0.0022 \\
2 & 225 & 0.0022 \\
3 & 237 & 0.0022 \\
\hline
\end{tabular}

Table 4: Supersonic Wind Tunnel Results Summary 


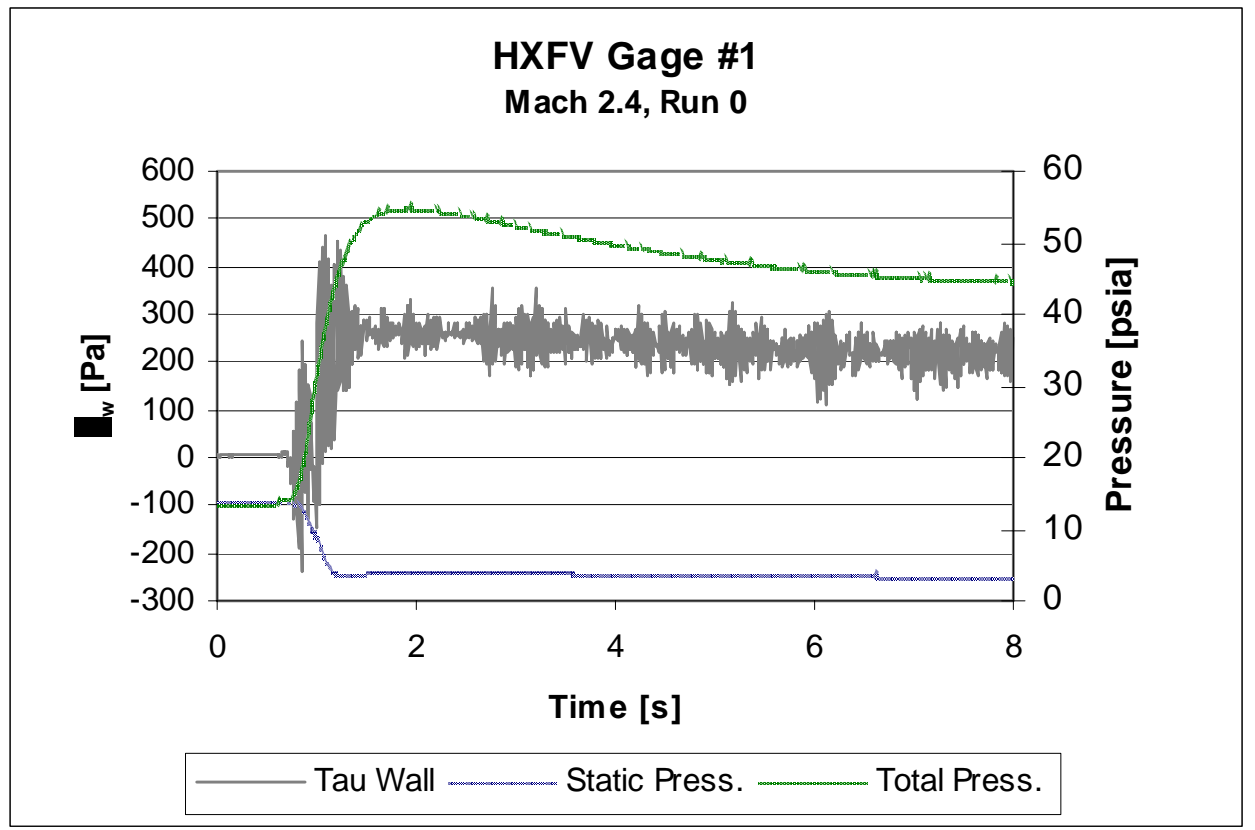

a)

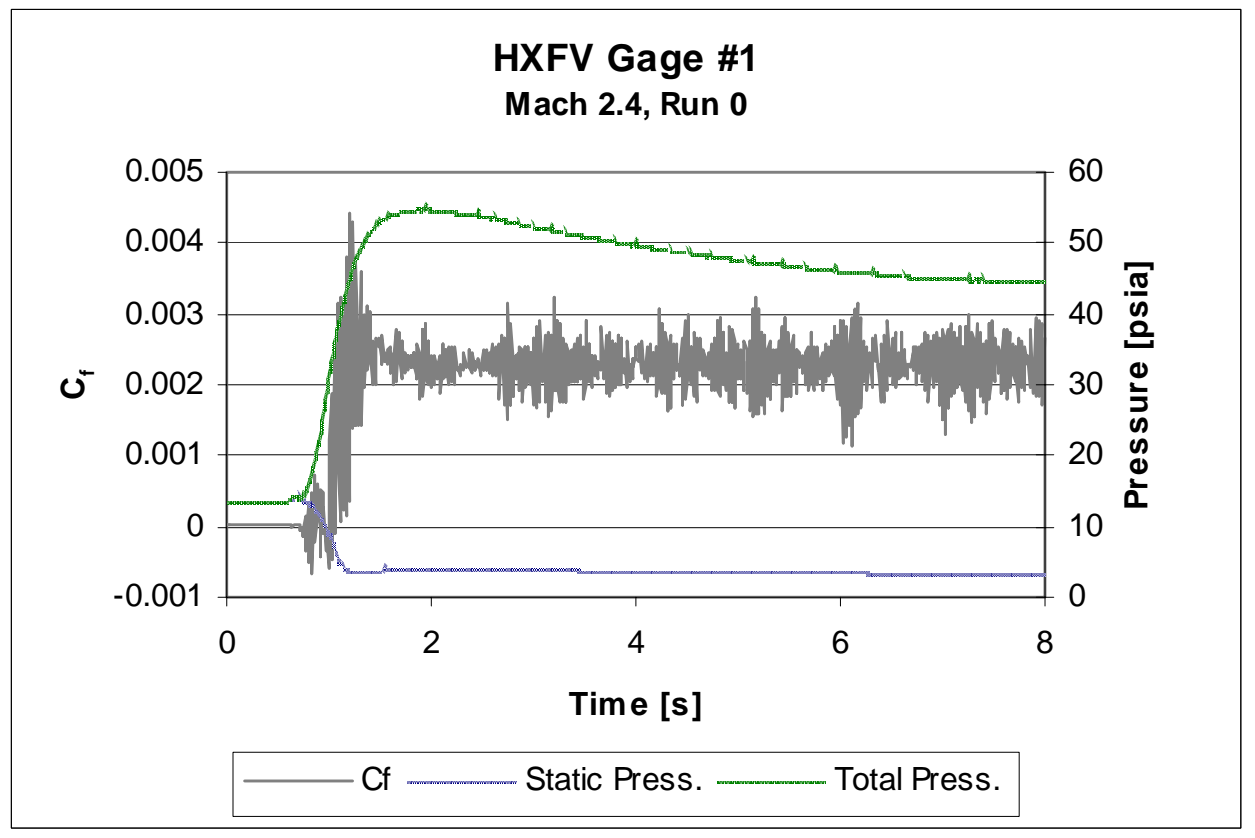

b)

Figure 83: Supersonic Wind Tunnel Test Results: Run 0

a) Shear Level b) Skin Friction Coefficient

tunnel produced large spikes and oscillations in the output that quickly damped out. From this it was concluded that filtering at a lower frequency was necessary for future wind tunnel tests. 


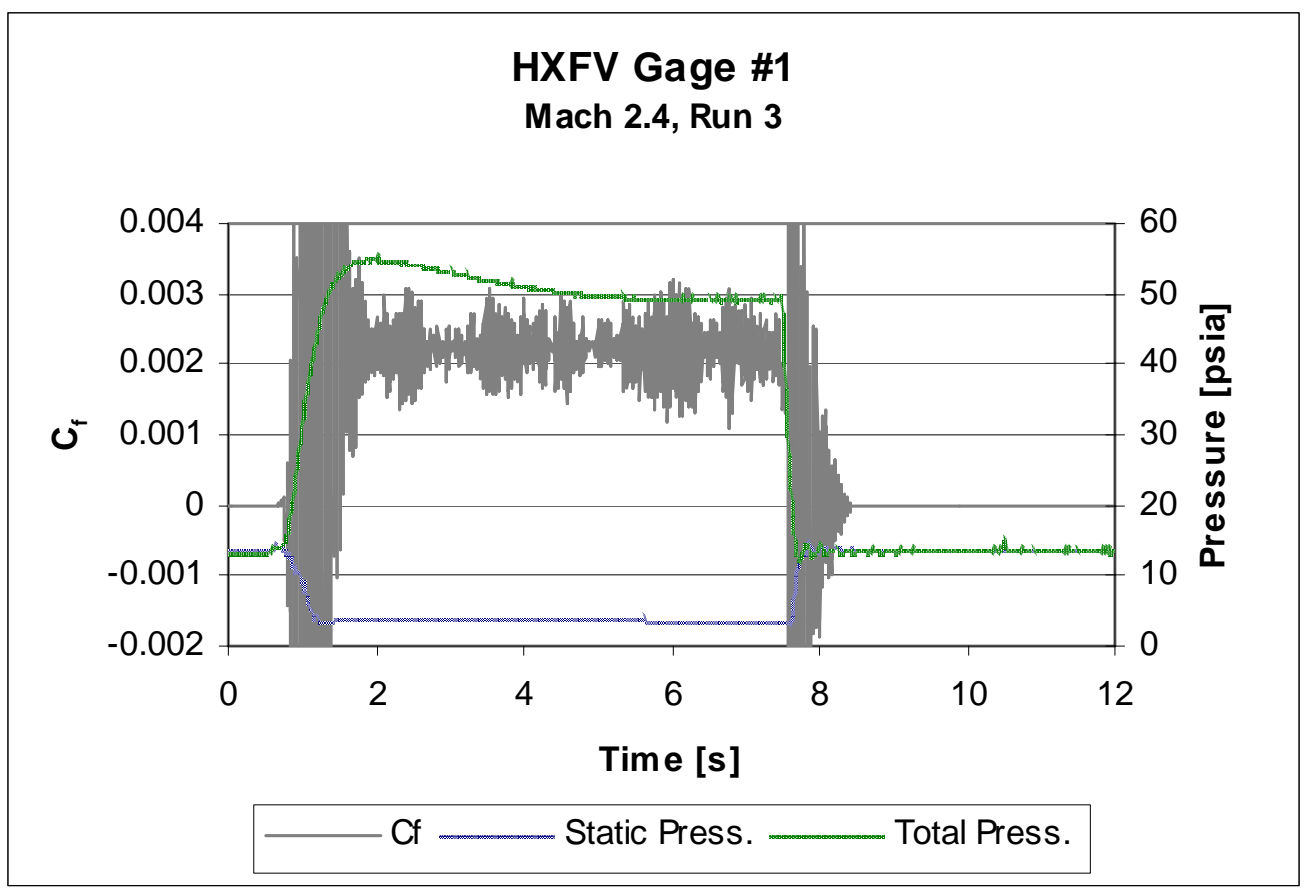

Figure 84: Supersonic Wind Tunnel Test Results: Run 3

These tests were very successful. The output was very consistent from run to run, as seen in Table 4. The output began and ended at zero with no shift due to temperature or anything else. The results obtained were close to the estimates for $\mathrm{C}_{\mathrm{f}}$ of 0.0018 to 0.002 .

Since this gage was very different from the previous skin friction sensors, this test was crucial in determining if the new features would work. The flexure ring proved to be capable of functioning as the flexible member in a direct measuring skin friction gage. It was also important to verify that a skin friction gage could operate without gap filler. This skin friction sensor was not filled with oil or any other substance, yet it was able to measure the skin friction coefficient within $10-15 \%$ of the estimated range of values. The higher $\mathrm{C}_{\mathrm{f}}$ of the experiment may be due to the location of the sensor not far downstream of the diverging section of the nozzle, where the positive pressure gradient causes boundary layer thinning. The results were also in good agreement with the measurements of the earlier skin friction sensor designs (compare Table 4 to Table 2). 


\subsection{HXEM Tests}

The cold flow tests proved that the skin friction sensor could accurately measure skin friction in a simple supersonic cold flow. The next step was to verify that the skin friction gage was capable of measurement in a scramjet flow environment. Therefore, before installation on the Hyper-X Mach 10 flight vehicle, it was tested in a wind tunnel ground test of the Hyper-X Engine Model (HXEM). This was the same full-scale partialwidth model of the Hyper-X engine on which the second skin friction gage was tested. Since that design failed to measure in the scramjet test environment, it was necessary for this gage to prove that it could do the job in the wind tunnel in order to proceed towards the goal of installing on the flight vehicle.

\subsubsection{Test Facility}

The facility used for these tests was the NASA Langley Arc-Heated Scramjet Test Facility (AHSTF). This facility was described in detail in Section 5.4.1. The wind tunnel and the engine model (HXEM) were the same as for the previous tests. The reader is referred to the previous discussion for a description of the wind tunnel operating capabilities and procedures.

The same wiring configuration as shown in Figure 51 was used again for this skin friction gage. The excitation for this gage, however, was 10 volts, and the gain factor was 500. A 2310 signal-conditioning amplifier provided the excitation and amplification. The signal from the 2310 was acquired by the facility NEFF system, as was the signal from the thermocouple built into the skin friction gage housing. Both signals were sampled at $50 \mathrm{~Hz}$ and filtered at $1 \mathrm{~Hz}$. The facility equipment, rather than the 2310, performed the filtering of the signal from the skin friction sensor.

The gage was mounted on the inlet ramp of the engine, as was done in the earlier tests of the second design. Again this was because there was no internal room for the sensors to be mounted in the combustor of the engine. For these tests there was no watercooling to the skin friction sensor.

The earlier tests in the AHSTF were conducted to simulate the enthalpy of Mach 7 flight. For these tests, however, NASA's test program called for Mach 6.5 flight enthalpy. The nozzle area ratio was the same as before, producing a Mach 6.0 freejet at the nozzle exit. The nominal total pressure was raised from 460 psia (3172 $\mathrm{kPa})$ for the 
previous tests to $555 \mathrm{psia}(3827 \mathrm{kPa})$ for these tests, and the total enthalpy was reduced from $1055 \mathrm{Btu} / \mathrm{lb}$ for the previous tests to $900 \mathrm{Btu} / \mathrm{lb}_{\mathrm{m}}$ for these tests.

There was also one slight modification to the skin friction gage that allowed it to be tested in the HXEM engine. This modification was necessary for these wind tunnel tests, but was not present during any of the other tests on this gage. This modification also was not a part of the skin friction gage to be installed on the flight vehicle. The modification consisted of a half-inch spacer that fit between the upper surface of the housing and the engine wall. The stainless steel adaptor was also lengthened by half of an inch. This extra length and the spacer were necessary because of the way in which the copper engine wall plates were mounted to the frame of the engine. This frame is made of half-inch thick steel and has cutouts for instrumentation and other devices that require access to the backside of the copper engine wall plates. The cutout where the skin friction sensor was to be mounted was not large enough to accommodate the entire housing. Using the spacer and extending the length of the adaptor provided the clearance necessary for the housing. A sketch of the modified components is shown in Figure 85.
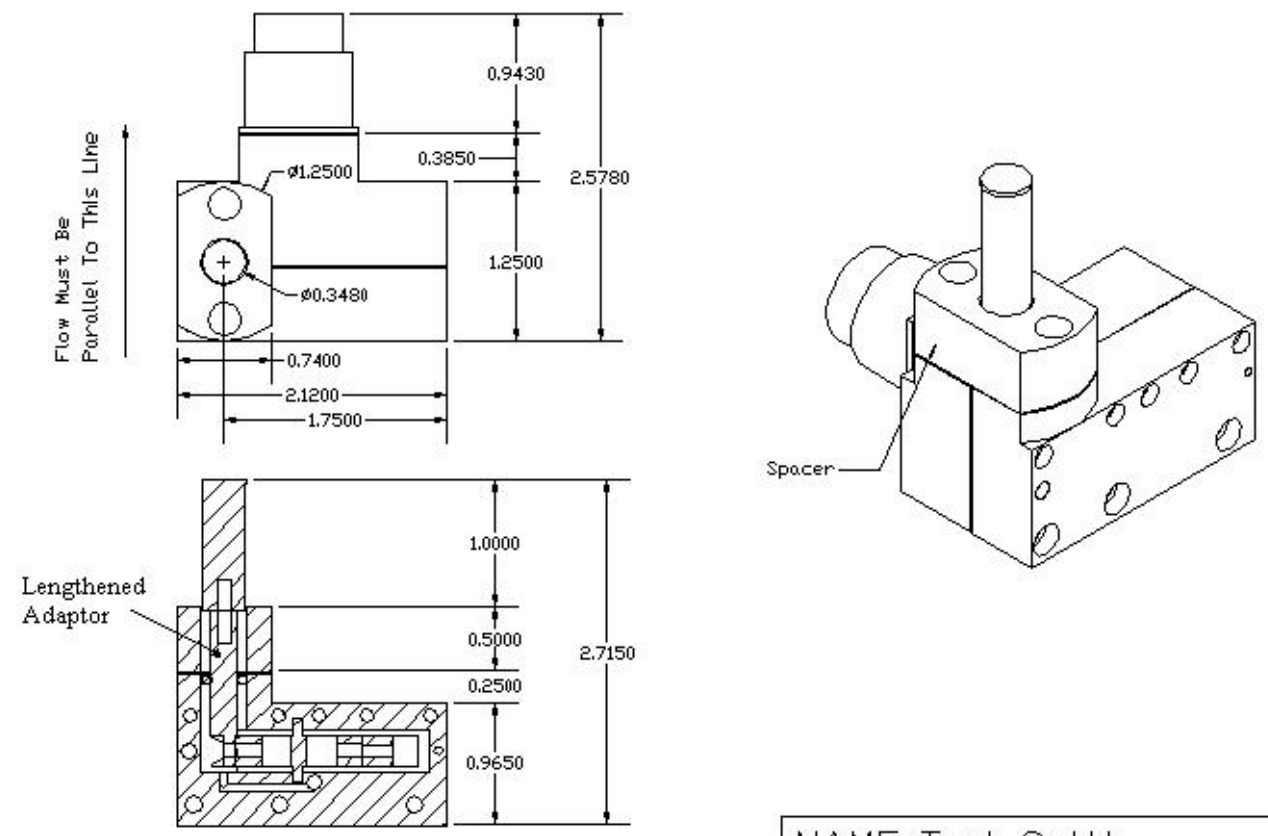

Figure 85: Modification for HXEM Tests 
The tunnel operation sequence was as follows. The main and bypass air were opened and allowed to stabilize for a couple of seconds. Then the arc power was turned on for a total of about 30 seconds of hot, high enthalpy flow. The bypass air was closed off 5 seconds after the arc power was off, with the main air following about 3.5 seconds later. The tunnel condition for the shutdown sequence is unknown. With the arc off and the bypass air off, the flow is due to the main air alone. The flow from the nozzle exit may or may not be supersonic, and without heating it is likely to be very cold with possible condensation of the air.

\subsubsection{Test Results}

A total of six high-enthalpy runs were performed with the Design Three skin friction gage design in the HXEM. The results of these tests are shown in Figure 86 through Figure 91. In addition, there were two runs in which the arc power did not come on. The tunnel condition for this situation is unknown, so the results of these are not shown. The first run was a trial run in which the arc was only powered for about five seconds. All the other runs followed the run procedure noted above, with the arc on for a total of about 30 seconds. This test procedure results in a relatively long exposure to the high-enthalpy, high heat flux flow. The flight test, in comparison, is only planned to expose the engine to 7 to 15 seconds of hot flow.

The output from the skin friction sensor was extremely repeatable for all of the runs. The output clearly followed the tunnel events, with jumps in output as the tunnel came on and again as the arc came on. There was also repeatable thermal drift in all of the runs. However, the magnitude of the thermal drift was not so great as to overwhelm the shear signal. It was still quite easy to determine the changes in the signal due to the shutting down of the arc and the air. The thermal drift was minimal early in the run, and increased during the run. Instead of returning to zero, there was an offset in the output that continued to rise as the heat soaked into the engine after the end of the run. This delay can also be seen in the temperature history of the gage shown in Figure 92. The temperature of the gage remained nearly constant well into the run, with an increase of a few degrees near the end. The temperature of the gage at the start of each consecutive run was higher than the previous run. This indicated that the temperature continued to rise after each run as the heat soaked through the engine walls. Differences in the amount 
of change in gage temperature that occurred between the runs are accounted for by differences in the amount of time between runs (from under 30 minutes to over an hour). Runs that were conducted close together show a high jump in temperature between them because the heat soaked into the engine but did not have time to begin dissipating away again before the next run, as occurred when more time was taken. Also, between test 133.04 and 133.06 a cold run (no arc) was performed. The extremely cold air helped to cool the engine walls, lowering the temperature seen at the start of test 133.06.

Earlier results of the temperature tests had shown that temperature changes caused output from the strain gages, but did not affect the sensitivity of the gage. This was verified by the experience in the HXEM. Though there was thermal drift between the start and the end of each run, the voltage changes at the startup and shutdown were the same magnitude. Also, the skin friction gage temperature rose steadily during the test day. The skin friction gage began the day at room temperature, but during the last test the gage temperature was about $250^{\circ} \mathrm{F}\left(121^{\circ} \mathrm{C}\right)$. Yet, the output from the sensor was consistent between all of the runs.

The fact that the sensitivity remained constant meant that the signal due to shear could be obtained by simply subtracting out the part of the signal due to thermal drift. The delay in the temperature rise and drift made it possible to determine the shear level from the data early in each run. Since the flow on the inlet was steady, any change in the shear signal during the steady portions of the run could be attributed to thermal drift. So, assuming the signal should have been constant between facility events (start-up, arc on/off, shutdown), the thermal drift portion of the signal was separated out by subtraction. A polynomial was then fit to the thermal drift, and this was then subtracted from the original signal. For example, in Test 133.04, the initial shear value after the arc came on and before drift was about $72 \mathrm{~Pa}$. This value was subtracted from the signal for the entire hot portion of the run, and the remaining signal level was attributed to thermal drift. A polynomial was then fit to the drift and subtracted from the original signal.

In Figure 86 through Figure 91, the original signal, the polynomial drift curve, and the corrected signal are shown for each run, along with the total pressure. It is clear when comparing the charts that the skin friction gage gave extremely similar results in each run. The corrected signals consistently showed steady shear levels of about 75-85 
Pa during the hot flow test time, and the corrected signals all returned to zero. In every run there was interference in the signal between about 8.5 and 10 seconds, and again between about 34 and 36 seconds. The cowl actuation motor that opened and closed the engine cowl at the beginning and end of each hot test period caused this. This was quite a large motor, and it produced electromagnetic interference during operation. During each run there was also a period during the shutdown phase between the cut-off of the bypass air and the cut-off of the main air. During this period the output of the gage was generally erratic. The tunnel flow condition for this phase of operation is not documented, so this was not a concern.

The shear levels were normalized by dynamic pressure to calculate the skin friction coefficient. This was done with two different methods. For the first calculation the dynamic pressure at the exit of the facility nozzle was used. For the second calculation, the local dynamic pressure (obtained from pressure measurements and oblique shock calculations of Mach number) was used. The results are summarized in Table 5, which shows the shear level and the skin friction coefficient for each run. The values of $\mathrm{C}_{\mathrm{f}}$ based on the tunnel nozzle dynamic pressure ranged from 0.0015 to 0.0018 . When the local dynamic pressure was calculated, the $\mathrm{C}_{\mathrm{f}}$ range was 0.0009 to 0.0011 .

NASA performed a computational prediction of the shear on the inlet of the scramjet wind tunnel model. The predicted levels of shear were about 85-90 Pa. The results of the experiment agree with this prediction within 15\%. See Appendix C.4 for more details regarding the CFD solution.

\begin{tabular}{|c|c|c|c|}
\hline Test & $\tau_{\mathrm{w}, \text { avg }}(\mathrm{Pa})$ & $\mathrm{C}_{\mathrm{f}, \text { qn ozzle }}$ & $\mathrm{C}_{\mathrm{f}, \text { qlocal }}$ \\
\hline 133.01 & 74.9 & 0.0016 & 0.0009 \\
133.02 & 82.2 & 0.0017 & 0.0010 \\
133.03 & 87.3 & 0.0018 & 0.0011 \\
133.04 & 72.0 & 0.0015 & 0.0009 \\
133.06 & 87.3 & 0.0018 & 0.0011 \\
133.07 & 77.0 & 0.0016 & 0.0010 \\
\hline
\end{tabular}

Table 5: AHSTF Test Results Summary 


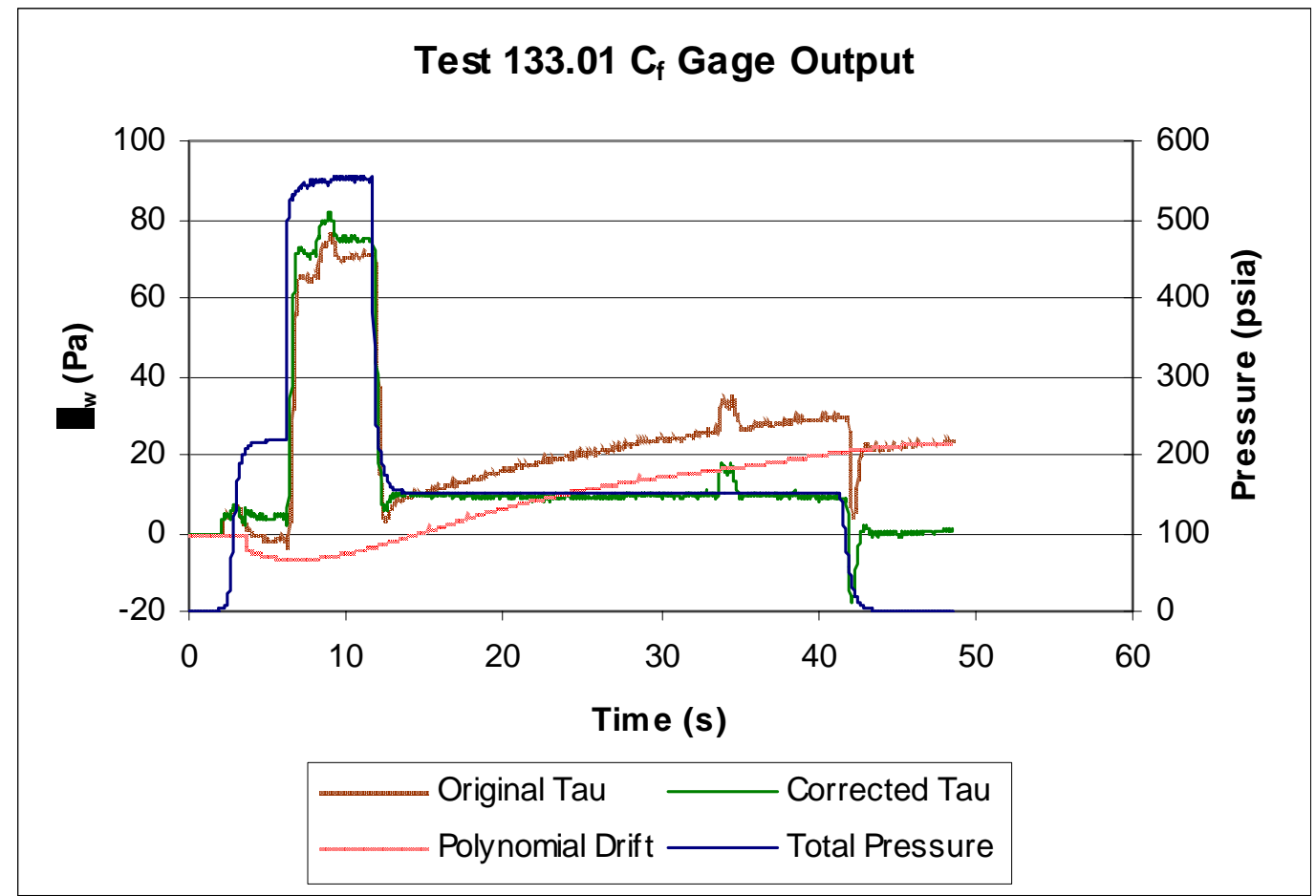

Figure 86: AHSTF Test Results: Run 133.01

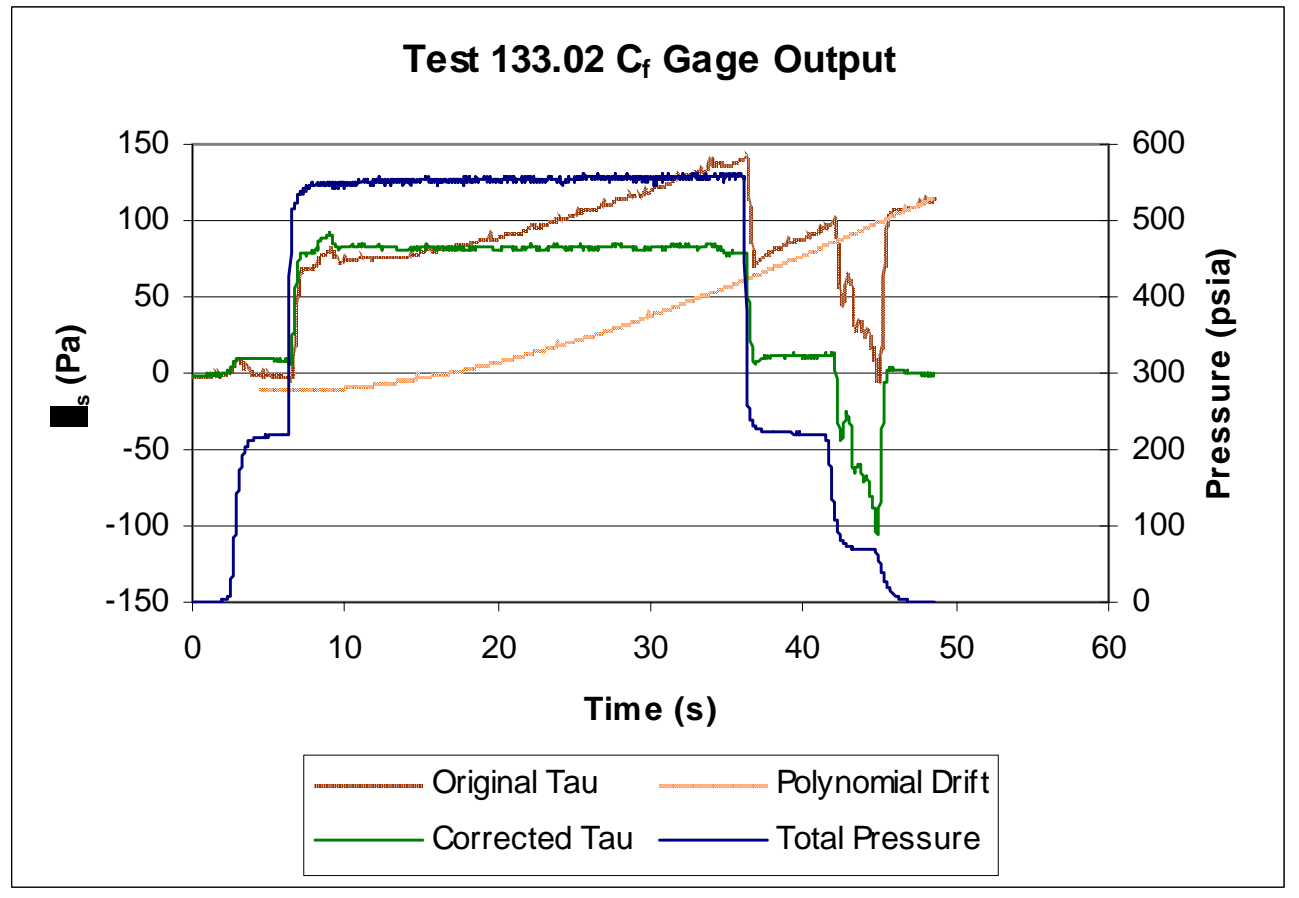

Figure 87: AHSTF Test Results: Run 133.02 


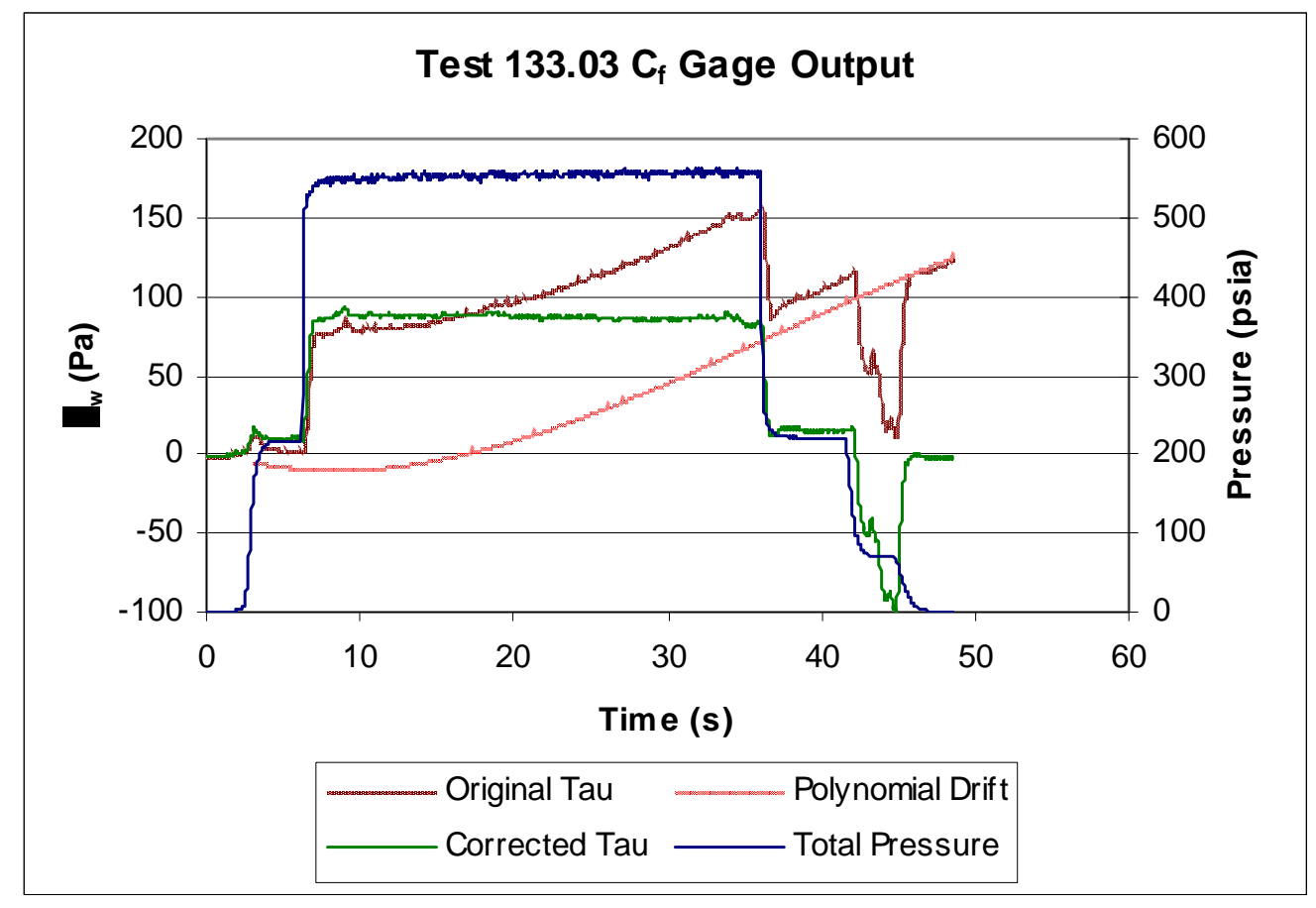

Figure 88: AHSTF Test Results: Run 133.03

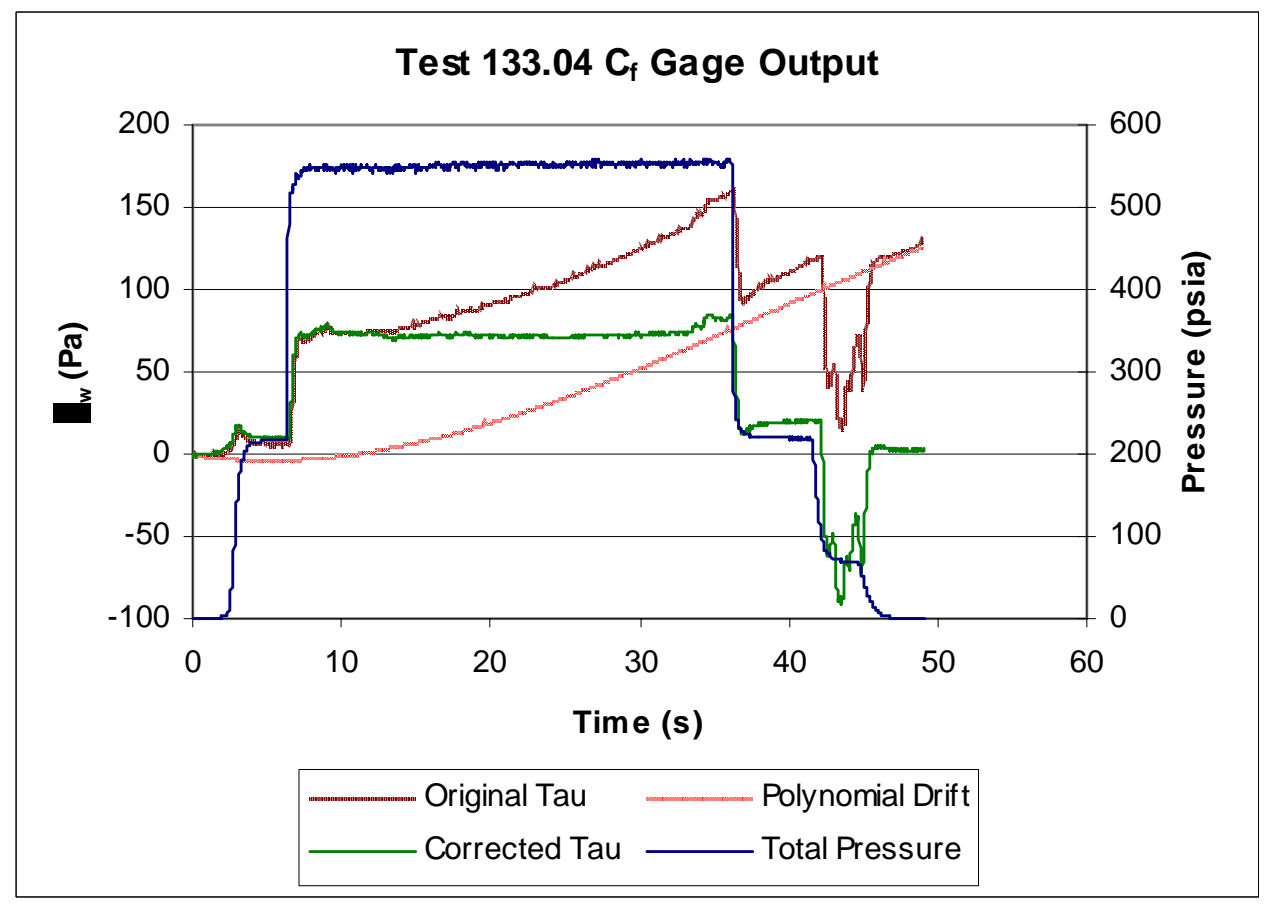

Figure 89: AHSTF Test Results: Run 133.04 


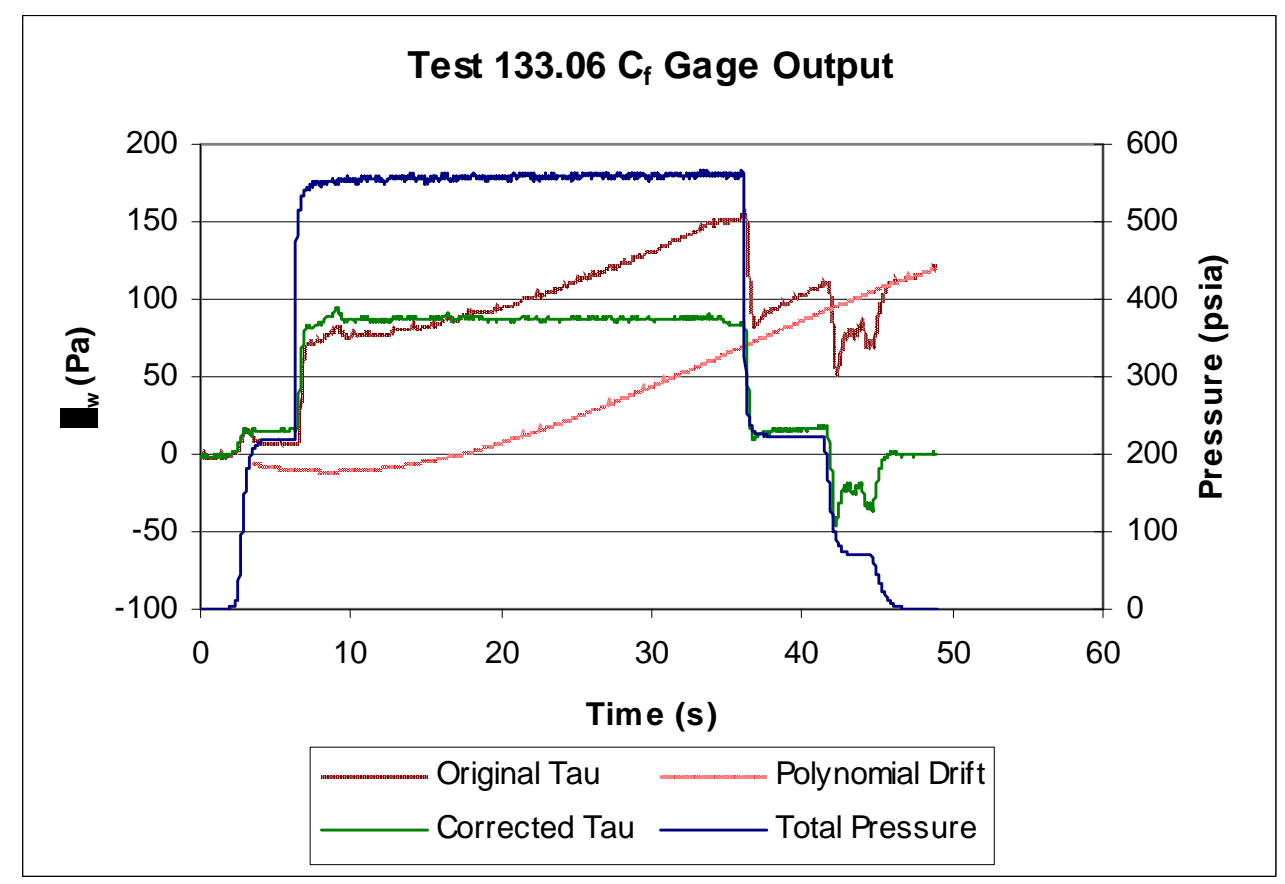

Figure 90: AHSTF Test Results: Run 133.06

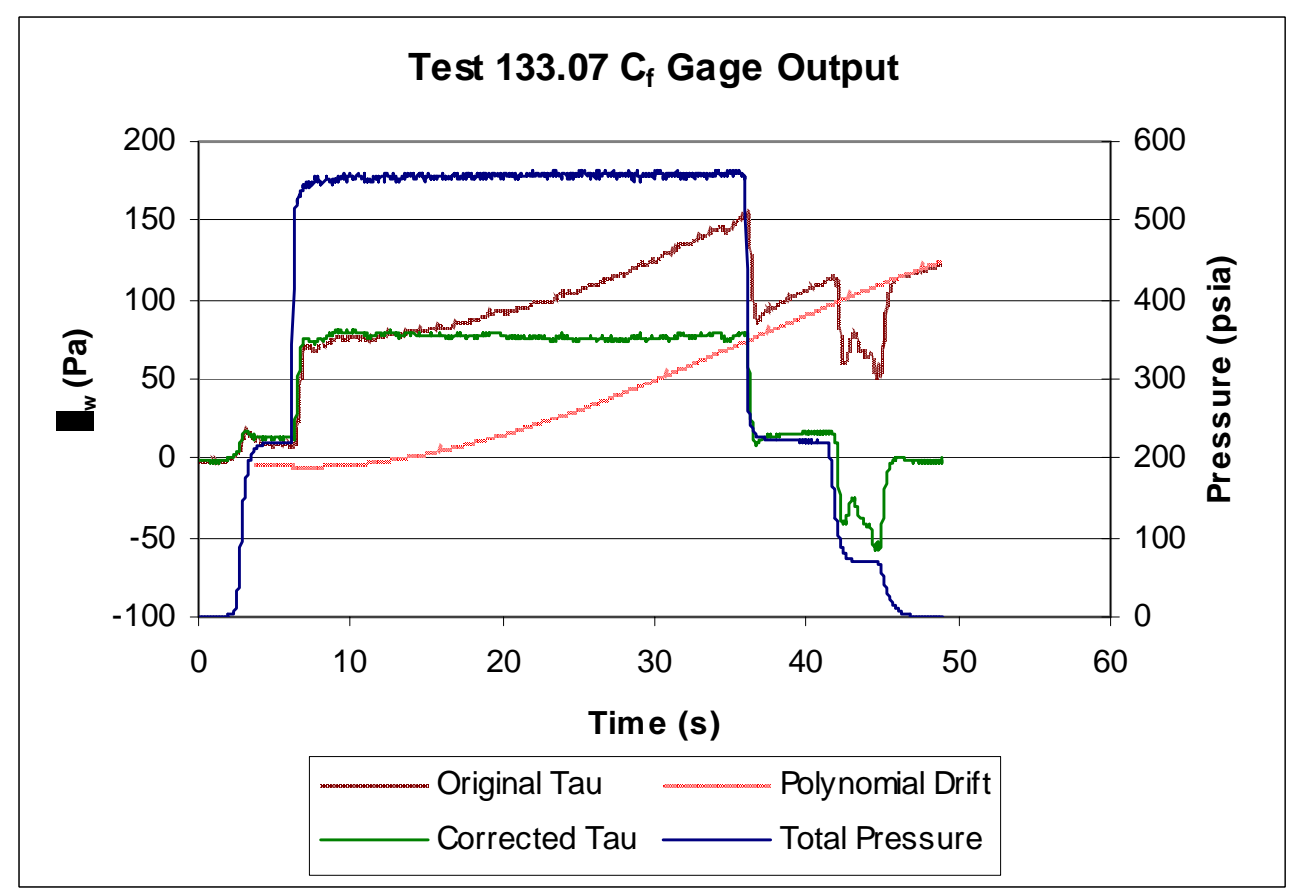

Figure 91: AHSTF Test Results: Run 133.07 


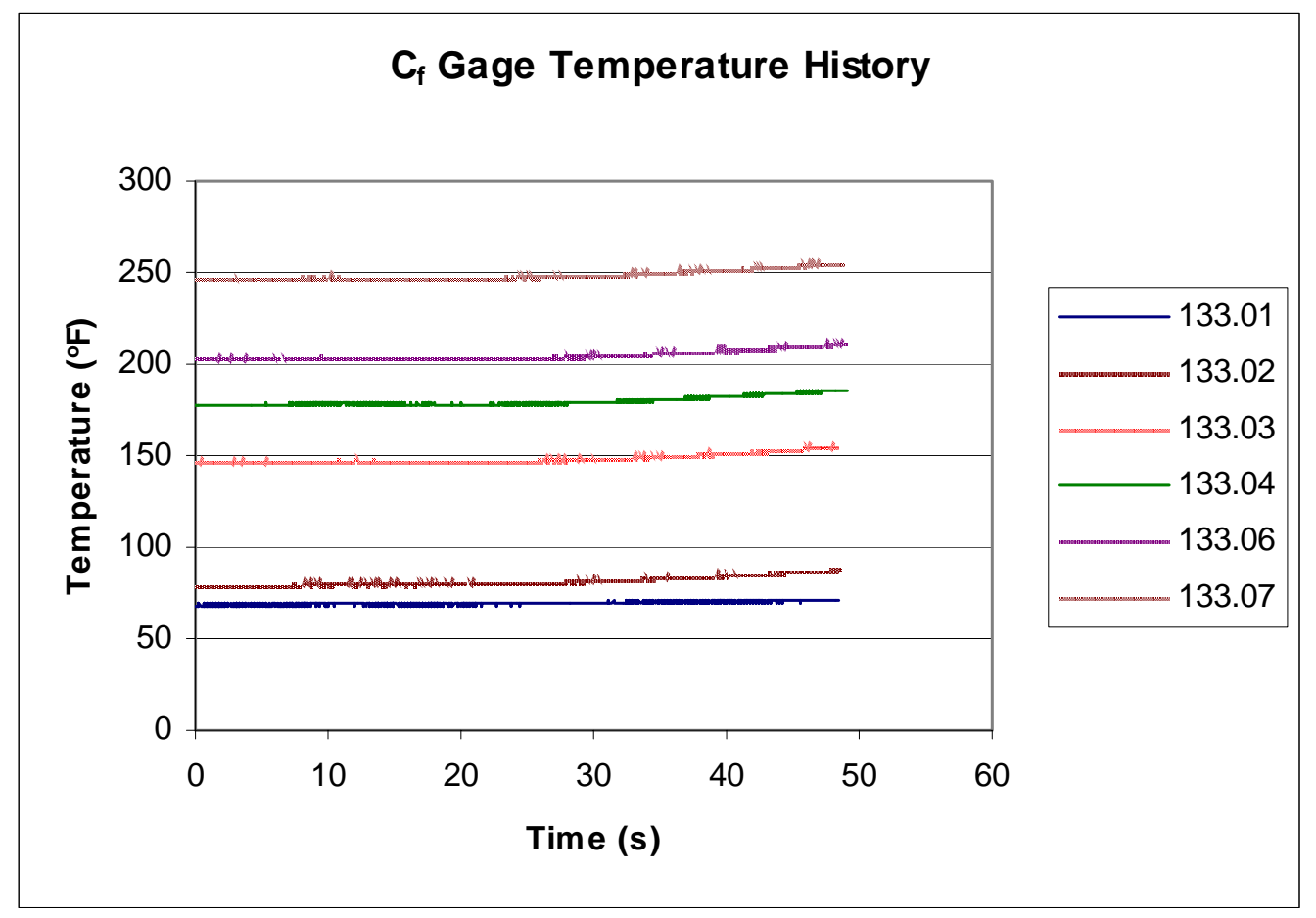

Figure 92: AHSTF Test Results: Gage Temperature History

\subsection{Concluding Remarks About Gage Design Three}

The tests in the Hyper-X Engine Model were the climax of the development program for the Hyper-X flight vehicle skin friction sensor. The tests in the HXEM provided the closest simulation of the flight vehicle environment possible on the ground. In these tests it was determined if the new design features of the gage would work in the high-speed, high-enthalpy supersonic flow of the scramjet engine.

The HXEM tests showed that an un-cooled skin friction sensor without oil fill could be used for testing on a scramjet engine. This was made possible largely by the foil strain gages in conjunction with the flexure ring. Though it was not possible to entirely eliminate thermal output from this gage, the thermal output was small enough to be accounted for with relative ease. This would not have been possible with semiconductor strain gages. Experience with the semiconductor strain gages showed much greater sensitivity to temperature than the foil strain gages. The design of the gage, with its thermal barriers, also helped to delay the onset of thermal drift until well into the hot test time. This is important since the flight vehicle test will be much shorter than the wind 
tunnel test. There will be even less exposure time to the high heating levels. It should be possible to determine the shear levels in the flight test from the first several seconds of testing, before thermal drift affects the signal. And, even after thermal drift occurs, it should be just as easy to account for it by looking at the jumps in the output as was done in the HXEM tests.

There will, of course, be other factors on the flight vehicle. The accelerations and changes in angles during the flight are also of concern. However, the vehicle state data acquired during the flight should help in determining the angles at various points during the flight. Then the angular calibration of the skin friction gage can be used to subtract out any output due to angles. This shouldn't be much of a problem during the engine test, though, which should take place under straight and level flight conditions.

The HXEM tests also provided insight into the response of the skin friction gage to axial accelerations. Fuelling during the HXEM tests produced sudden changes in the axial force of up to $50 \mathrm{lb}_{\mathrm{f}}(222 \mathrm{~N})$. There was no response from the skin friction gage due to these force changes. The counterweight of the gage effectively reduced the sensitivity of the gage to such accelerations.

After the HXEM tests the skin friction gage was again inspected, and no visual damage was seen. The gage was calibrated to see if the any change in the sensitivity had occurred. The result of the calibration after the test was within $3 \%$ of the pretest calibration. The sensitivity of the sensor was not significantly changed by the wind tunnel tests.

It has been effectively demonstrated that this skin friction sensor design is suitable for testing on the Hyper-X Mach 10 flight vehicle. See Appendix C for official NASA reports documenting the qualification of this gage. The environmental pressure and temperature tests, the vibrational tests, the heat loads tests, and the HXEM tests were all successfully performed with this gage design. All that remains at the time of this writing (mid-August, 2001) is for the final approval and the installation in the flight vehicle engine. The primary goal of the project was achieved by developing a very different skin friction sensor than previous designs. The new key features- foil strain gages and flexure ring, no water-cooling, and no oil fill- made the gage more robust, a prerequisite for flight-testing. 


\section{CHAPTER SEVEN:}

\section{HEAT FLUX GAGE DESIGN FOR HYPER-X FLIGHT VEHICLE}

The goal of this research was to produce a skin friction gage to be installed on the third Hyper-X Research Vehicle (HXRV), designated X-43A. During the development of the skin friction sensor for the Hyper-X, a need for heat flux sensors was realized for the Hyper-X flight vehicle engine. The skin friction and heat flux to the engine wall are related to each other by the Reynolds Analogy. The Reynolds Analogy for simple, lowspeed flows is well established. However, it is not as well documented in complex, hot, high-speed flows such as the scramjet engine. Therefore, in order to investigate this relationship, it was desirable to also measure the heat flux in the engine at locations near the skin friction sensors. To achieve this goal, a heat flux gage was designed that would make use of the same mounting holes as the skin friction sensor. The heat flux gage discussed below was the final product designed, tested, approved, and installed on the Hyper-X flight vehicle.

\subsection{Gage Design}

The heat flux gage design was simple in concept. The gage consisted of a copper plug that fit into the same mounting hole as the skin friction sensor. The copper plug had a one-inch $(2.54 \mathrm{~cm})$ cylindrical shaft, and a 1/4-inch flange. The flange was the same shape as the flange of the first two skin friction gage designs. Clearance holes for the \#1/4-20 mounting screws passed through the flange, providing the mounting mechanism for the gage. A sketch of the copper plug is shown in Figure 93.

Accommodations for three thermocouples were machined into the copper plug. The first thermocouple was a fast-response, coaxial Type $\mathrm{T}$ thermocouple manufactured by Medtherm, Inc. that mounted flush with the flow surface at the top of the plug. This thermocouple consists of a constantan lead wire (0.011-inch diameter) coated in ceramic insulation 0.0005 -inch $(12.7 \mu \mathrm{m})$ thick. Next, a copper tube is swaged over the coated constantan lead wire. A very thin layer of chromium is then vacuum deposited over the end of the probe to provide the junction between the two metal leads. This surface, 

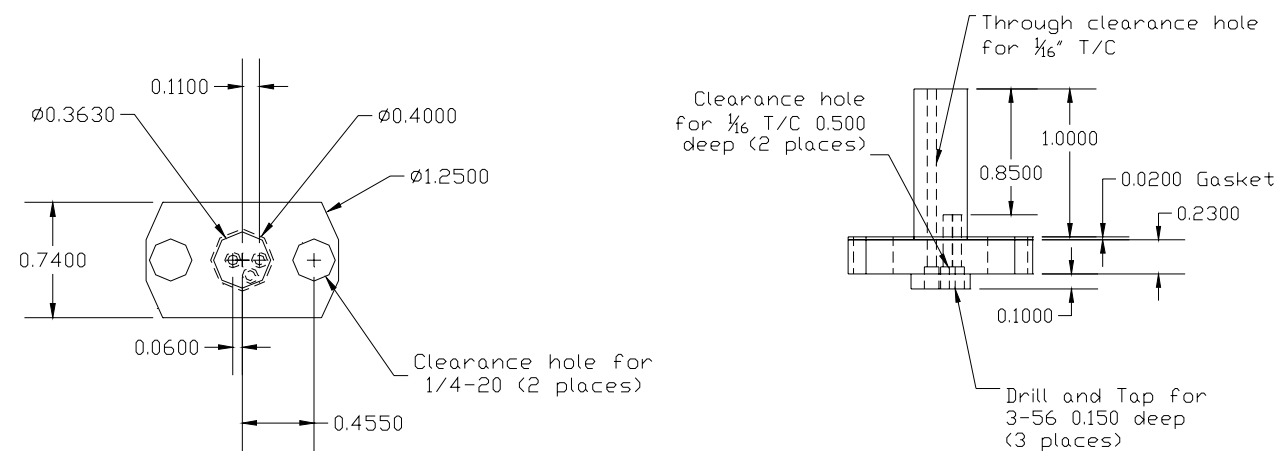

Figure 93: Heat Flux Copper Plug Sketch

which is 1 to $2 \mu \mathrm{m}$ ( 0.00004 to 0.00008 inches) thick, is the key to the fast response (on the order of microseconds) of the thermocouple ${ }^{46}$. The low thermal mass of this junction plating ensures that the heat into the plating will be quickly transferred to the thermocouple leads. A second copper shaft surrounded the thermocouple leads and was insulated from them. This provided electrical insulation of the thermocouple from the surrounding copper plug. A sketch of the coaxial thermocouple is given in Figure 94.

Type $\mathrm{T}$ thermocouples were chosen because of the match in material properties of the thermocouple to the copper plug. The property $\mathrm{k} \rho \mathrm{C}$ (where $\mathrm{k}$ is the thermal conductivity, $\rho$ is the density, and $\mathrm{C}$ is the heat capacity) is the most important parameter to match when choosing a coaxial thermocouple. The product $\mathrm{k} \rho \mathrm{C}$ is taken as the average for the different materials layers of the thermocouple ${ }^{47}$. Type $\mathrm{T}$ thermocouples

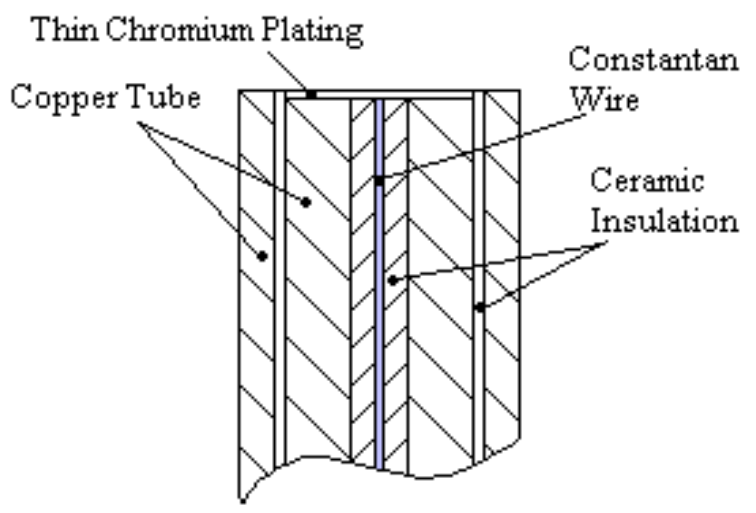

Figure 94: Coaxial Thermocouple Sketch (not to scale) 


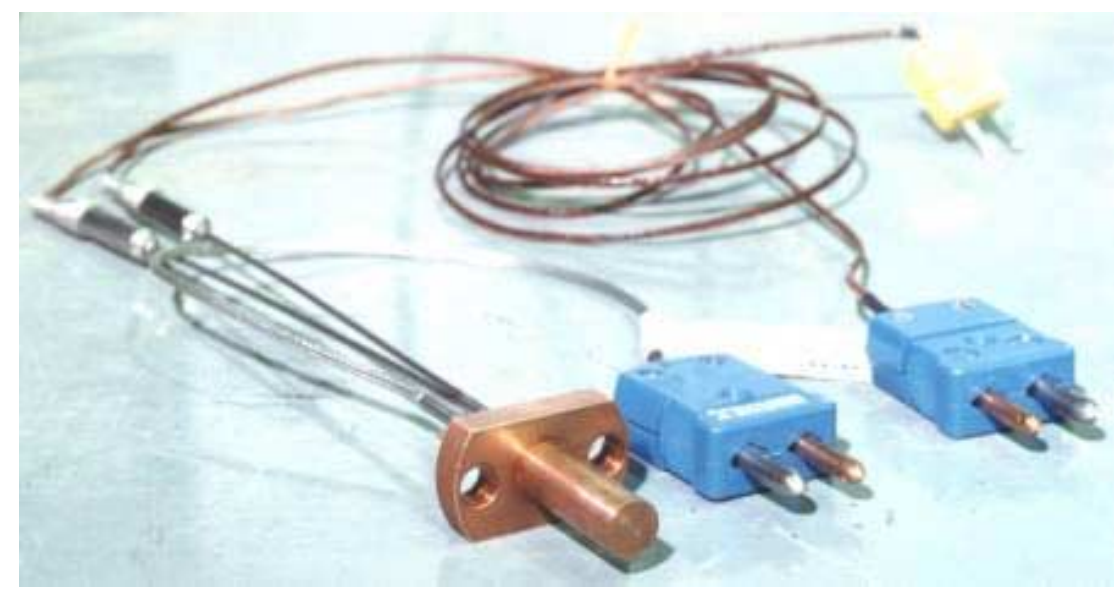

Figure 95: Assembled Heat Flux Gage (Connectors Shown to Identify Thermocouples: Blue-Type T, Yellow-Type K)

are made from copper and constantan leads. The outer thermocouple tube and the insulating tube are both made of copper, and the thin center wire is constantan. Since the constantan wire and the ceramic insulation layers are so small, the average properties of the coaxial thermocouple are very close to the properties of the copper plug in which the thermocouple is mounted.

The two thermocouples mounted in the back of the copper plug were exposed junction thermocouples. These mounted next to each other inside the copper plug such that the junctions were in contact with the copper plug at a distance 0.85 inches (2.159 $\mathrm{cm}$ ) from the surface. One of these thermocouples was a Type T thermocouple, and the other was a Type $\mathrm{K}$ thermocouple. Figure 95 shows a completed heat flux gage.

Using Type $\mathrm{T}$ thermocouples was the best way to make the temperature measurements inside the heat flux sensor, and in a normal laboratory environment would have posed no difficulties. However, due to the nature of the data acquisition system available for the flight test, an alternative method for obtaining the temperatures in the heat flux sensor was derived that required the addition of the Type $\mathrm{K}$ thermocouple.

Instead of acquiring the two Type $\mathrm{T}$ thermocouples independently, it was necessary to connect them in series. The constantan leads were spliced together, and then the millivolt signal between the copper leads was acquired by the data system. The Type $\mathrm{K}$ thermocouple data was acquired normally and used as a reference temperature assumed to be the same as the backside Type $T$ thermocouple. Figure 96 illustrates the wiring scheme. 
Since the millivolt signal corresponds to the temperature difference between the surface and backside temperatures, post-processing was necessary to find the surface temperature. The calibration for Type $\mathrm{T}$ thermocouples gives the temperature as a seventh order polynomial function of the voltage from a Type $\mathrm{T}$ thermocouple $\left(\mathrm{T}=\mathrm{a}_{0}+\right.$ $\mathrm{a}_{1} \mathrm{~V}+\mathrm{a}_{2} \mathrm{~V}^{2}+\mathrm{a}_{3} \mathrm{~V}^{3}+\ldots$ ). A root-finding Fortran code was written to find the voltage from this calibration that would correspond to the temperature of the backside, as measured by the Type $\mathrm{K}$ thermocouple. The voltage computed by the program, which is the same as would be given by a Type $\mathrm{T}$ thermocouple measuring the backside temperature, was then added to the voltage acquired from the two Type $\mathrm{T}$ thermocouples in series. This new voltage then corresponds to the voltage that would be given by a Type $\mathrm{T}$ thermocouple measuring the surface temperature. Finally, by using this voltage in the seventh-order Type T thermocouple calibration polynomial, the surface temperature could be found.

The above procedure produced time histories of the surface and backside temperatures. The temperature difference across the heat flux gage was also obtained by

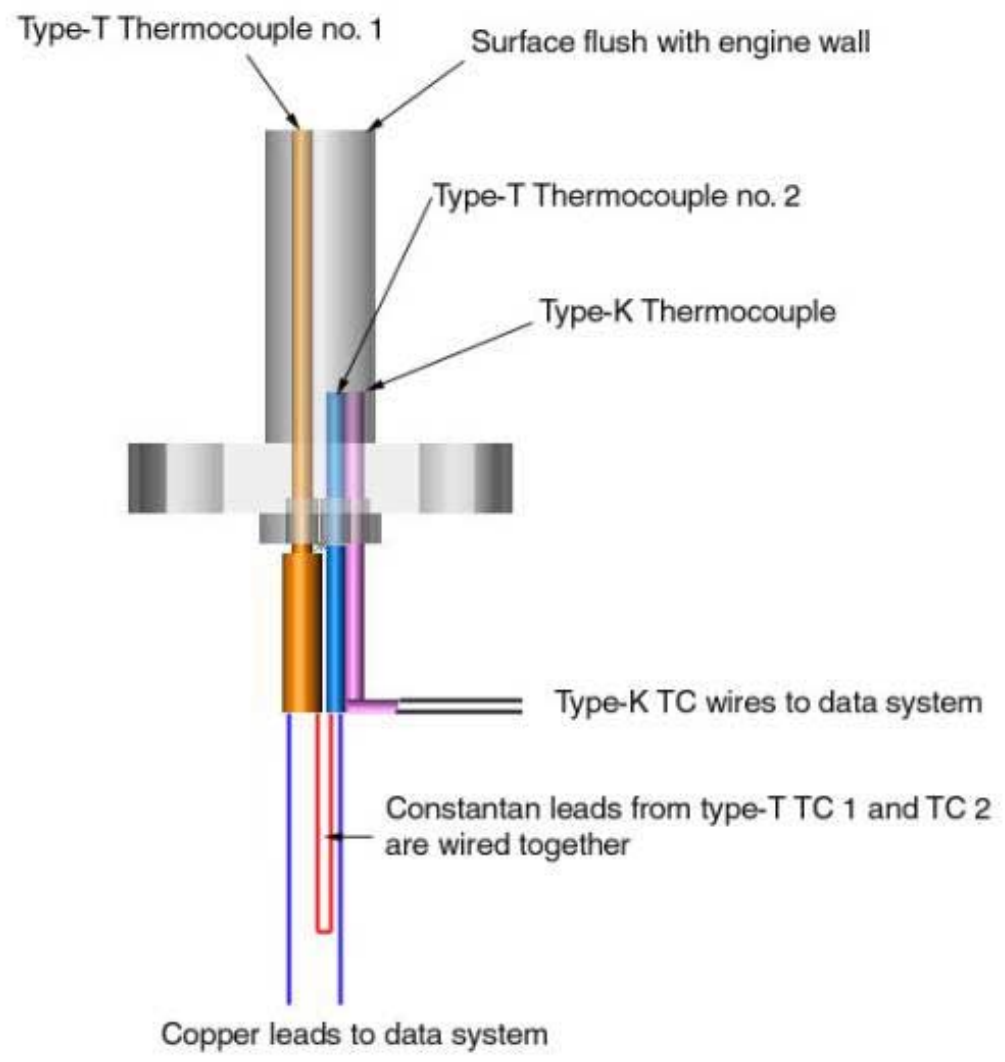

Figure 96: Wiring Schematic for Heat Flux Gage (From T. Bui ${ }^{48}$ ) 
taking the difference of the surface and backside temperatures.

In the overview of heat flux measurement techniques by Diller ${ }^{47}$, this configuration is categorized as a "Type 2 Method". Slug and null-point calorimeters are other examples of this type of sensor. No calibration is necessary for this type of heat flux sensor. Instead it operates on first principles. Taking the time history of the temperature, the problem is solved by calculating backward to find the heat flux necessary to produce that temperature history. One-dimensional transient conduction equations are often used assuming a semi-infinite solid. The calorimeter devices however, measure only a single temperature that is located below the surface of the gage. This limits the time response of these types of sensors. There is an initial transient response before the useful measurement begins. There is also a constraint on the length of the useful measurement period, which is limited by the time taken for the temperature to penetrate the total depth of the gage. By using a coaxial thermocouple, the time response of the heat flux sensor is much faster. Adding the second temperature measurement on the backside of the gage can also extend the useful measurement time. Using the backside temperature as a boundary condition and appropriately modeling the heat transfer essentially relaxes the semi-infinite assumption, allowing for the longer measurement time ${ }^{47}$.

The data reduction for these types of heat flux sensors is the most involved part of the measurement process. For a semi-infinite body, there is the well-known CookFelderman technique ${ }^{49,50,51}$, and several variations and modifications of it. These are called Class 1 Methods, and are limited in their ability to adequately model the physics of the problem ${ }^{52}$. A more detailed review of these methods is given by Diller ${ }^{53}$.

In response to the shortcomings of the Class 1 Methods, numerical (as opposed to analytical) techniques were developed for resolving the temperature distribution. These are called Class 2 Methods. These methods are better at modeling the physics of the problem, but have trouble resolving surface temperature gradients. See Walker and $\mathrm{Scott}^{52}$ for a more thorough discussion.

Class 3 Methods use an inverse technique. The heat flux into the sensor is assumed, and the temperature distribution is calculated. A least-squares comparison is then made between the calculated and measured temperatures. An iterative optimization 
scheme minimizes the difference between the measured and calculated temperatures to find updated heat flux guesses. These methods are capable of eliminating the effect of noisy data, and they do not amplify errors and/or noise in the data. These methods can also be extended to multi-dimensional problems ${ }^{52}$.

The data reduction method used for the heat flux sensor of this study falls into the third class, and has been developed by Walker ${ }^{52,54}$ and others. This particular method uses an axisymmetric conduction equation to solve for the temperature distribution inside the heat flux sensor, using known information about the geometry and thermal properties of the sensor. The backface temperature is added to the heat transfer model as a boundary condition. The inclusion of the backface measurement allows a longer useful measurement period. A previous successful test of this method was made using a coaxial and backside thermocouple pair in a turbine blade ${ }^{55}$.

\subsection{Pressure Tests}

Since this heat flux sensor was designed for the Hyper-X flight test, it also had to undergo environmental qualification tests. As with the skin friction sensor, these tests included pressure and temperature tests.

The pressure test was performed at NASA Dryden Flight Research Center. The heat flux gage was placed in a pressure chamber. The wires for the gage were routed through a pass-through in the wall of the chamber to multimeters so that data could be recorded during the tests. The Type $\mathrm{K}$ thermocouple was acquired by a thermocouple multimeter, and the leads from the two Type $\mathrm{T}$ thermocouples were wired in series and acquired as a millivolt signal on a regular multimeter. The chamber was then sealed and the pressure was reduced to correspond to different altitudes. Each altitude level was held for about 10 minutes before continuing to the next data point. It was planned to simulate up to $120,000 \mathrm{ft}$, but the pressure chamber was not able to go any further than $96,000 \mathrm{ft}$. The temperature in the chamber remained at ambient during the pressure tests.

Figure 97 shows the results of the pressure test. As can be seen, there was no change in the output of the heat flux sensor during the test. This was expected since only a temperature difference across the heat flux sensor should produce a signal. Visual inspection showed no change in the heat flux gage. These tests showed that the gage was 


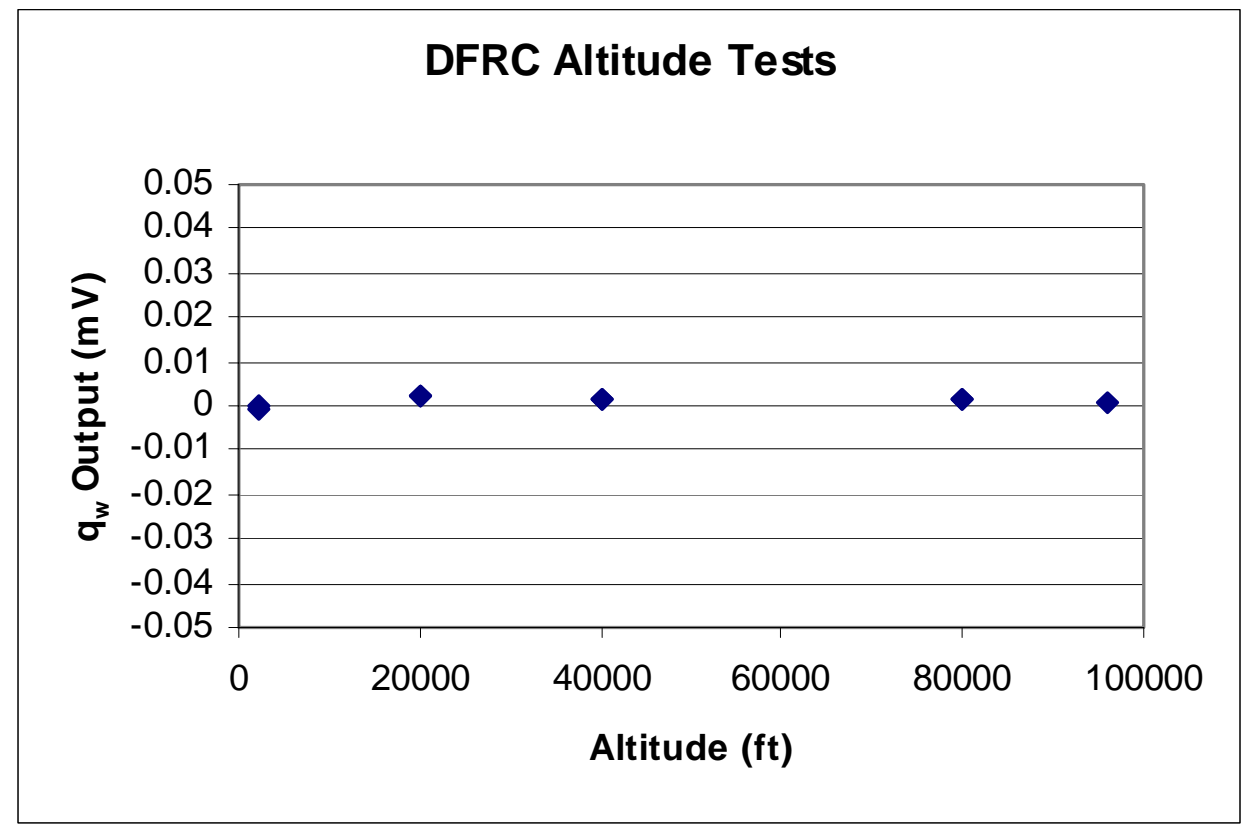

Figure 97: Pressure Environmental Qualification Test Results

not sensitive to pressure and could survive the altitude expected during the flight test without difficulty.

\subsection{Temperature Tests}

The heat flux gage was also tested for temperature sensitivity. These tests were to examine the ability of the gages to function at the temperatures expected during the flight test. Those temperatures range from a low of $-40^{\circ} \mathrm{F}\left(-40^{\circ} \mathrm{C}\right)$, which corresponds to the temperature at the altitude of the flight test, to a high of $160^{\circ} \mathrm{F}\left(71^{\circ} \mathrm{C}\right)$, which occurs when the vehicle is sitting on the tarmac before takeoff (NASA Dryden, where the flight is to take place, is located in the Mojave Desert in California).

The NASA Dryden pressure chamber was also used to do transient temperature tests. The heat flux gage was placed in the chamber at ambient pressure. The temperature of the heat flux gage was monitored using the Type $\mathrm{K}$ thermocouple in the backside of the copper plug. The output from the two Type $\mathrm{T}$ thermocouples wired in series was also monitored. The temperature of the heat flux gage fell slower than the air in the chamber, but faster than the skin friction sensor. This is due to the thermal mass of the sensor being less than the skin friction sensor. As the temperature of the gage fell, the temperature and output of the gage were recorded. 


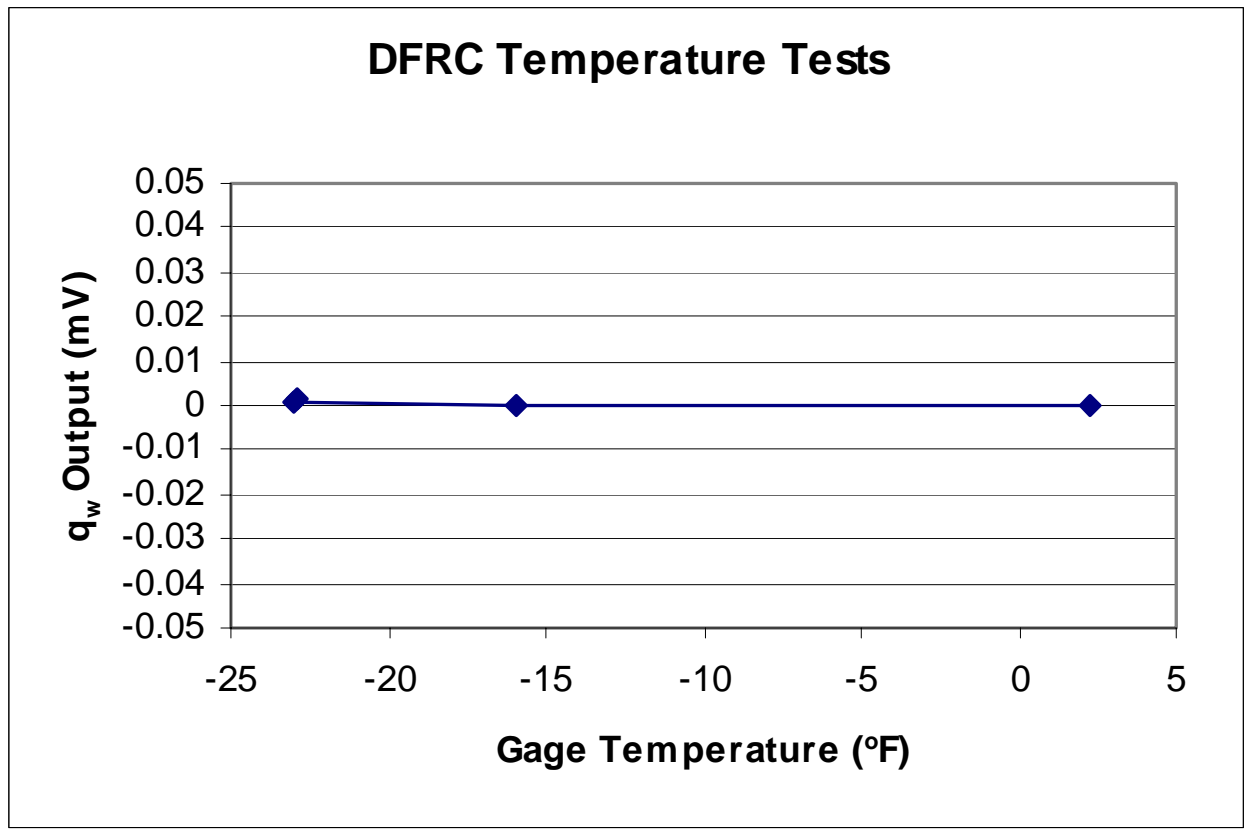

Figure 98: Transient Temperature Test

The result of this test is shown in Figure 98. Again, the output from the heat flux gage did not change with temperature. The high thermal conductivity of the copper plug makes it difficult for a temperature difference to be established between the surface and backside thermocouples. In a very slowly changing thermal environment such as the temperature chamber, the temperature across the heat flux sensor remains nearly constant. Therefore no output was produced from the two Type $\mathrm{T}$ thermocouples in series.

Steady state temperature tests were also attempted. These were done in the Virginia Tech Engineering Science and Mechanics Department using a Blue M Electric ETC-04D-C Environmental Test Chamber with a Pro-550 Controller. This test chamber has a two-stage cascade refrigeration system and is capable of testing at temperatures from $-80^{\circ} \mathrm{C}$ to $+190^{\circ} \mathrm{C}$. The gage was placed in the chamber with wires routed through a pass-through to a multimeter and an Omega Type $\mathrm{K}$ thermocouple DC millivolt amplifier. Output from the thermocouple amplifier was read on a multimeter and converted to temperature using the calibration for Type $\mathrm{K}$ thermocouples.

For each test point the test chamber was programmed to reach and hold a steady temperature. The gage was then allowed to reach the temperature of the chamber and remain there for about 45 minutes before moving to the next test point. 


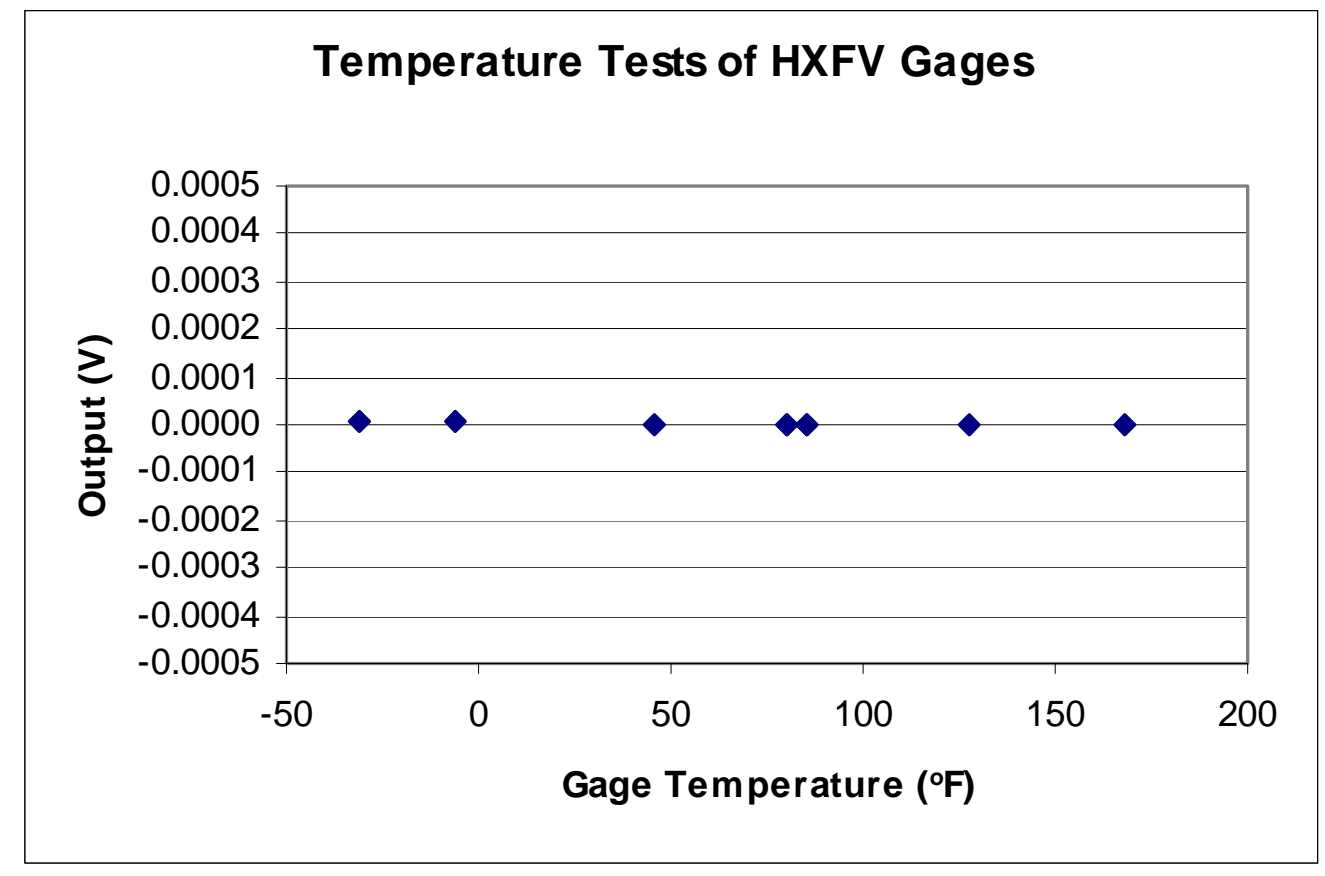

Figure 99:Steady State Temperature Tests

The result of these tests is shown in Figure 99. Like the tests in the Dryden test chamber, the output did not change with temperature. Even during the transient phases between temperatures in the chamber no temperature difference was established across the heat flux sensor. This result was expected, and demonstrated the great ability of copper to conduct heat quickly. This also indicated that the heat flux sensor would not be susceptible to erroneous output due to temperature as an interfering input.

\subsection{Heat Load Tests}

It was desired to test the heat flux gage in a heating environment closer to that expected in the scramjet engine during flight. To do this, it was necessary to reproduce the wall heat flux that was estimated for the engine near the location of the sensors. The same two facilities as were used for testing the skin friction sensor were used to attain this goal for the heat flux sensor. Both facilities used radiative heat flux sources. The heat to the engine wall during the flight test, however, will be transferred via convection. Methods for accurately reproducing high levels of convective heat fluxes are not available. The best way to accurately simulate high heating loads, then, is to do so with calibrated radiative systems. 


\subsubsection{NASA Dryden Tests}

The first set of heat load tests was performed at NASA Dryden Flight Research Center in the Flight Loads Laboratory. The facility was described in Section 6.6.1. As was discussed in that section, the heat loads tests there were not very successful. The heat flux level absorbed by the gage was not accurately known because the sensor surfaces were not coated with black soot. The heating was also not done with a step increase as was desired. An additional problem with the heat flux gage was discovered during these tests. During these tests, the output from the two Type $T$ thermocouples wired in series was much less than expected. When the two Type $\mathrm{T}$ thermocouples were acquired individually as thermocouples, the output was good and made sense. However, since the gage would be wired in series for the flight test, it was important to resolve this issue.

The coaxial thermocouple in the heat flux gage tested at NASA Dryden was not electrically insulated from the surrounding copper plug. This did not affect the thermocouple signals when acquired as individual thermocouples. But, when the two Type $T$ thermocouples were wired in series a parallel circuit was formed because both thermocouples were in contact with the copper plug. The copper plug itself became a resistor in parallel with the two thermocouples. The current flowing through the circuit was thus divided between the copper plug and the thermocouples, reducing the signal from the thermocouples. By replacing the coaxial thermocouple with one that was electrically insulated, as described earlier, this problem was solved.

\subsubsection{Vatell Tests}

The second set of tests was performed at Vatell Corporation in Christiansburg, Virginia. A flat plate heater facility very much like the one at NASA Dryden was used at Vatell to reproduce the predicted heat flux of the Hyper-X flight test. These tests provided the first real test of the heat flux gage in an environment close to the expected flight environment.

As described in Section 6.6.2, the tests at Vatell were better suited to simulate the heat load expected during flight. The sensor surfaces were coated with soot prior to testing. This gave the surface an emissivity of nearly 1.0, which meant that the amount of heat flux absorbed by the heat flux gage was nearly the same as was measured by the 


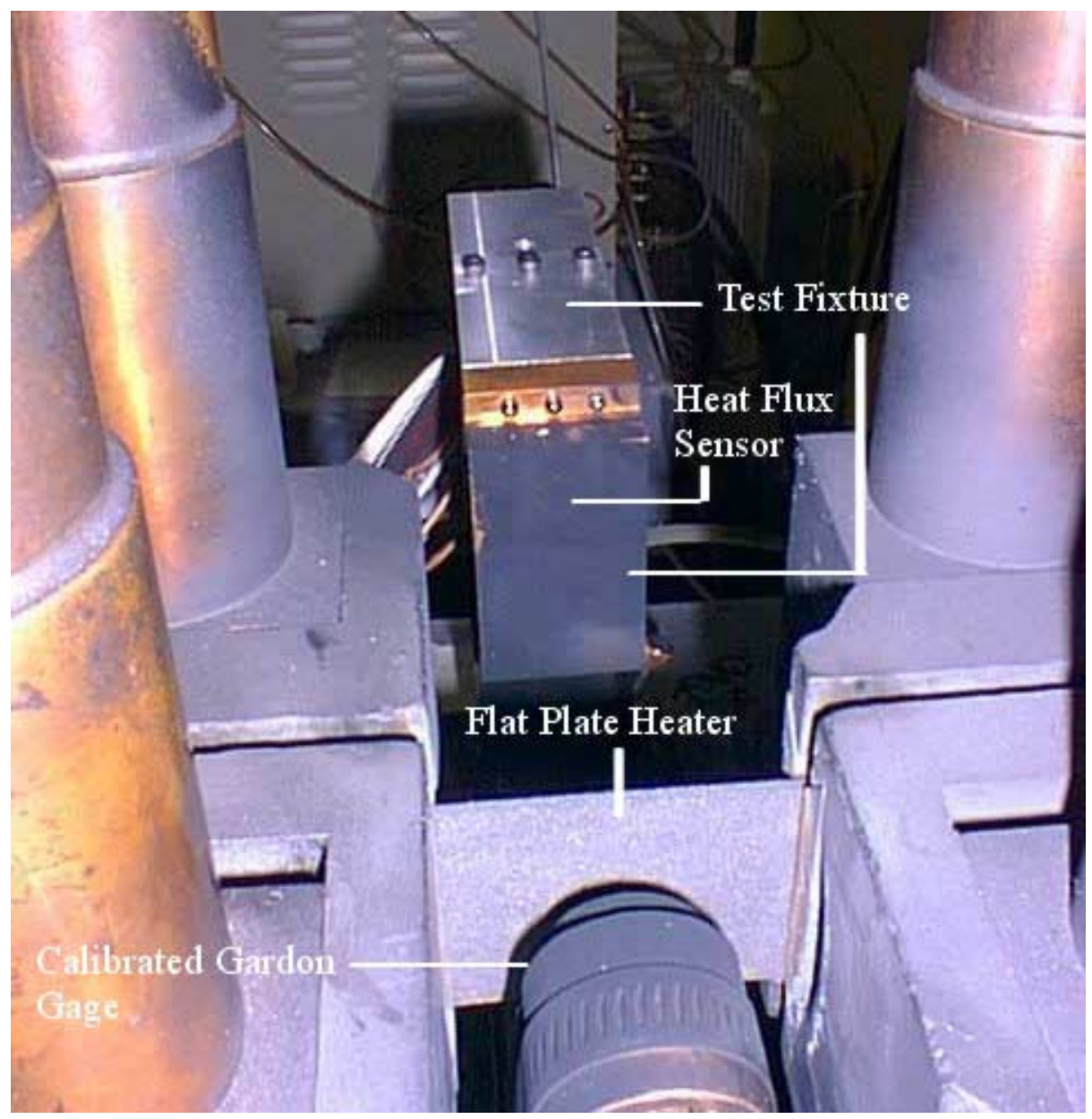

Figure 100: Heat Load Test Setup: Heat Flux Gage Mounted in Copper Block and Coated with Soot

calibrated Gardon gage on the opposite side of the flat plate heater. Figure 100 shows the heat flux gage mounted in the copper block and coated with soot prior to testing.

The step increase in heat flux could also be performed at Vatell. A traverse system allowed the heat flux gage to be pulled away from the heater plate and shielded during the ramp-up and shutdown periods. In this way the heat flux gage could be exposed to the heat load only for the brief amount of time expected during the flight test.

The heat flux sensor was tested at 30,70 and $100 \mathrm{Btu} / \mathrm{ft}^{2} \mathrm{~s}$. The output from the Type $\mathrm{T}$ thermocouples wired in series and the Type $\mathrm{K}$ thermocouple in the housing were recorded on a PC via a National Instruments A/D board using LabView data acquisition software. While the heater plate was ramped up to the test point the heat flux gage and copper mounting block were covered with a tin sheet and traversed on rails away from plate. Once the flat plate reached the designated heat flux, the shielding was removed 


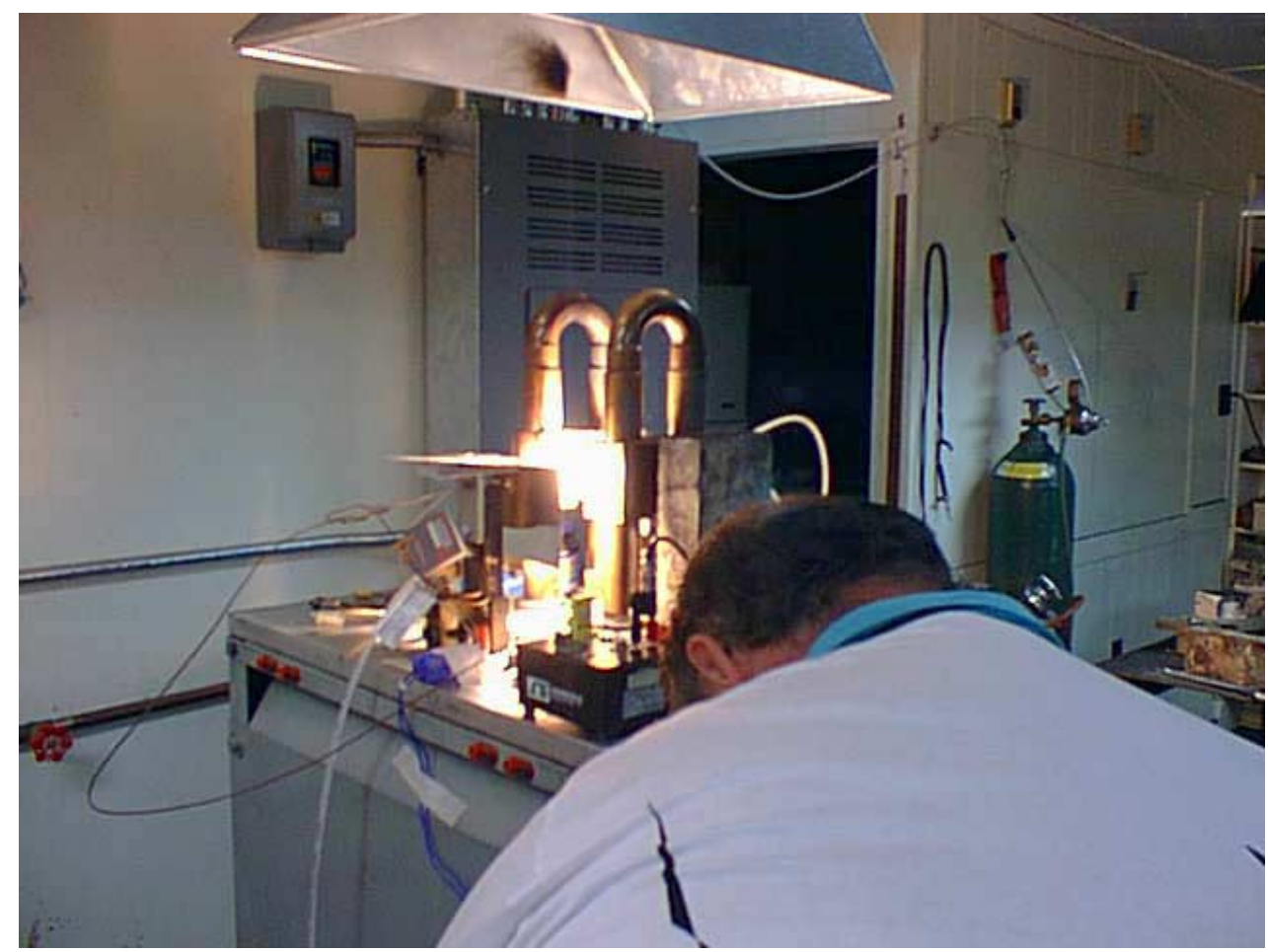

Figure 101: Heat Load Test Setup: Shielded Gage During Heater Ramp-Up

and the gage was traversed up to the flat plate for several seconds. Then the gage was traversed away from the plate again and the power to the heater plate was cut. Figure 101 shows the gage withdrawn from the plate and shielded as the flat plate heats up.

Results of the first of these tests are shown in Figure 102. Here the heat flux level was $30 \mathrm{Btu} / \mathrm{ft}^{2} \mathrm{~s}$. Figure 102 shows the surface and backside temperatures, as well as the difference between them, over time. The backside temperature was measured by the Type $\mathrm{K}$ thermocouple mounted in the back of the copper plug. The previously discussed method was used to convert the voltage from the two Type $\mathrm{T}$ thermocouples into the surface temperature. Several seconds of data were recorded before and after the exposure period. Before the exposure, the temperature was steady at room temperature as seen in the chart. At about 17 seconds into the run, the shielding was removed and the gage was moved within range of the flat plate heater. The gage was in the test position until about 25 seconds into the run, for a total exposure time of about eight seconds. Then the heater was powered down and the gage was withdrawn from the plate. At the start of the exposure period, the surface temperature rose quickly. The rate of temperature rise decreased slightly after the initial startup, but continued nearly linearly. The surface temperature reached a maximum of about $180^{\circ} \mathrm{F}\left(82^{\circ} \mathrm{C}\right)$. A slight delay was seen in the 


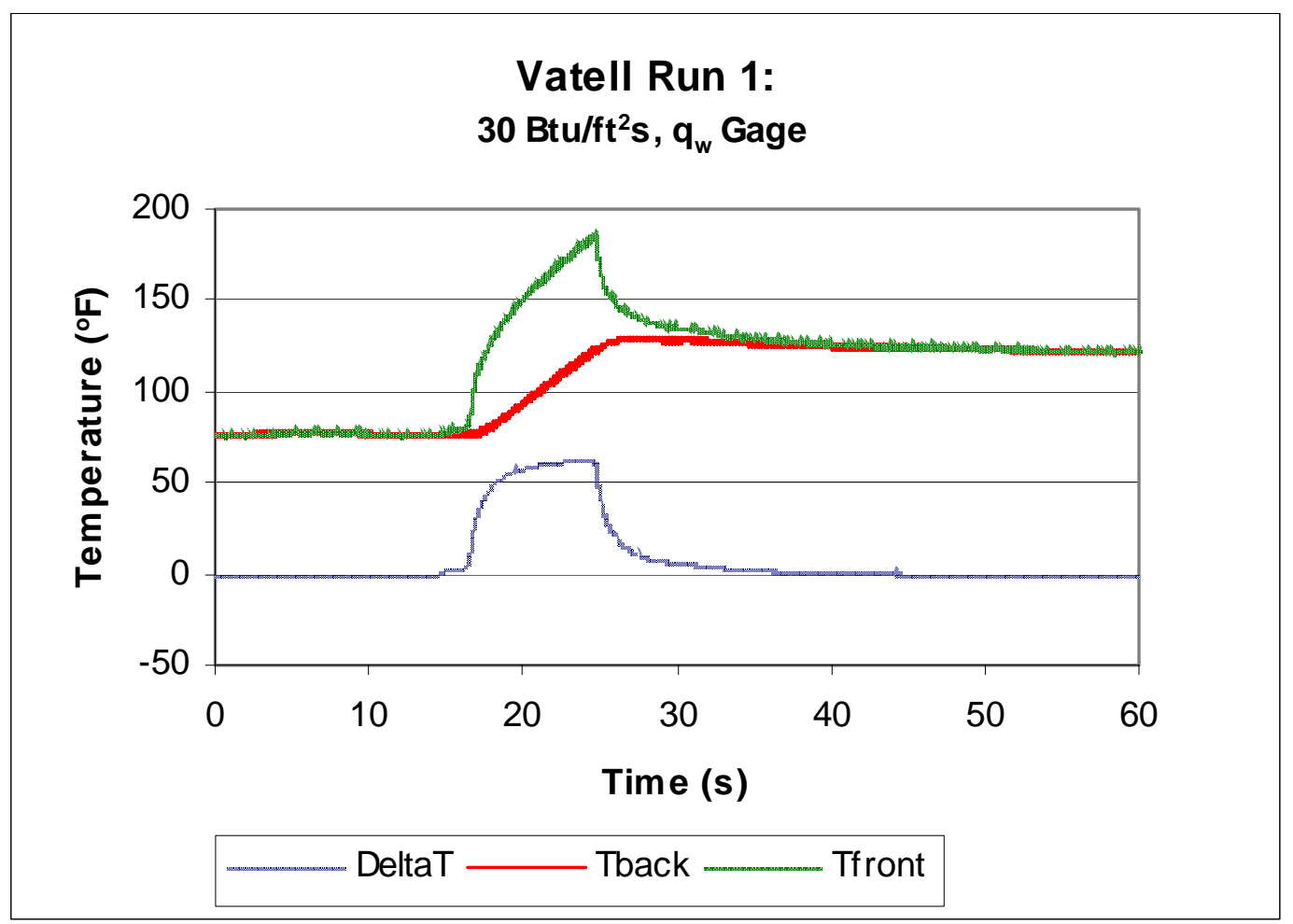

Figure 102: Heat Load Test Results: $30 \mathrm{Btu} / \mathrm{ft}^{2} \mathrm{~s}$

initial response of the backside temperature, as was expected. The backside temperature response followed that of a calorimeter exposed to a constant level heat flux, with a linear rise in temperature. The backside temperature reached a maximum of about $130^{\circ} \mathrm{F}$ $\left(54^{\circ} \mathrm{C}\right)$ shortly after the shutdown of the heater plate. The difference between the surface and backside temperatures rose sharply at the beginning of the test, approached a constant value of about $60^{\circ} \mathrm{F}\left(15.6^{\circ} \mathrm{C}\right)$, and then sharply fell towards zero at the end of the test.

Results of the second of these tests are shown in Figure 103. For this test the heat flux was $70 \mathrm{Btu} / \mathrm{ft}^{2} \mathrm{~s}$. Again the backside temperature, surface temperature, and temperature difference are presented in Figure 103. The heat flux exposure period for this test can be seen from the increase and decrease in the surface temperature. The beginning of the exposure period was at about 20 seconds into the run, and the end of the exposure period was at about 25 seconds, for a total test time of about 5 seconds. The backside and surface temperatures both began at about $83^{\circ} \mathrm{F}\left(28^{\circ} \mathrm{C}\right)$. Similar to the first test, the surface temperature responded before the backside temperature. The surface temperature again rose sharply at first, with a slightly slower rate of increase following. The maximum temperature of the surface was about $270^{\circ} \mathrm{F}\left(132^{\circ} \mathrm{C}\right)$. Again the backside 


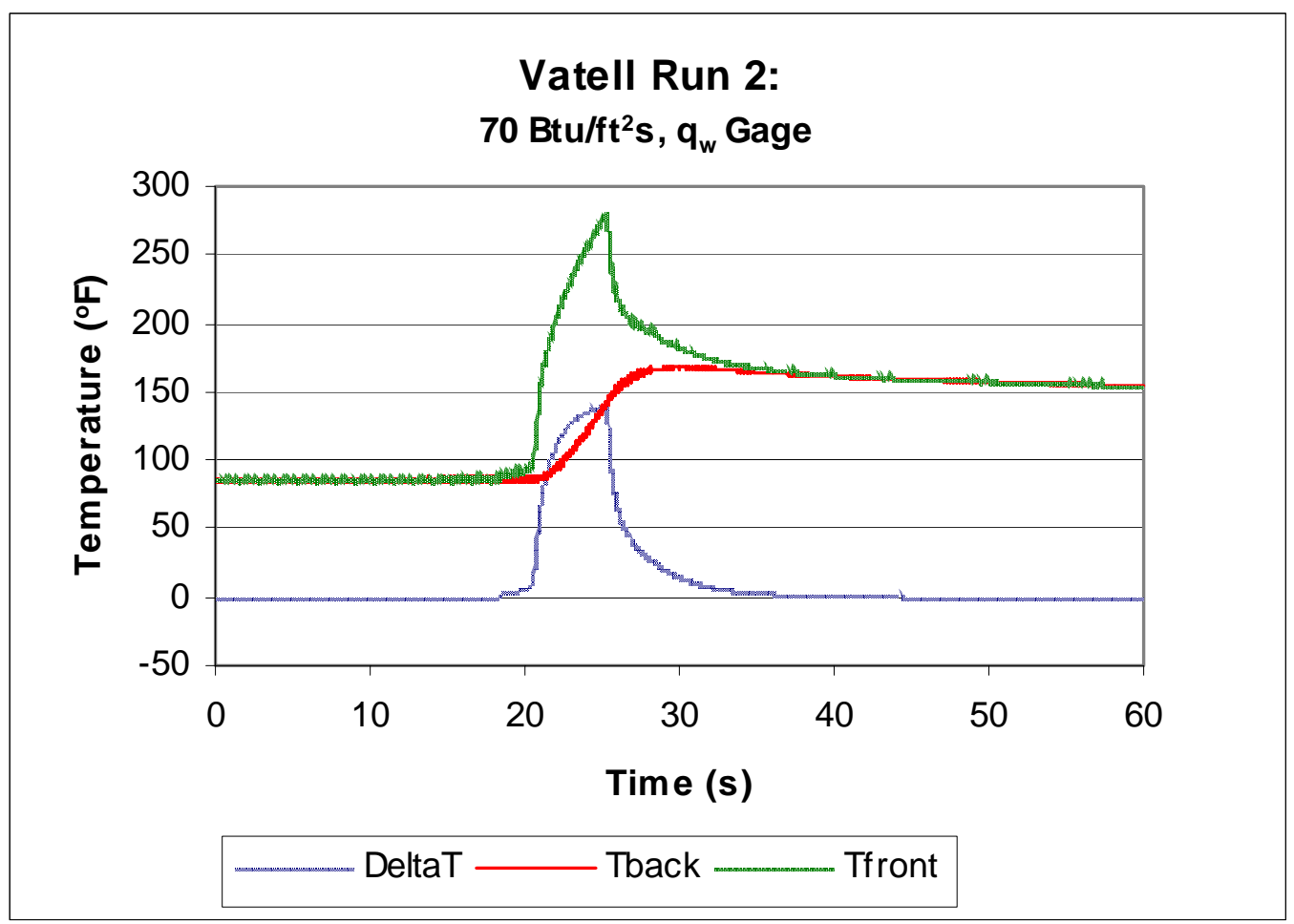

Figure 103: Heat Load Test Results: $70 \mathrm{Btu} / \mathrm{ft}^{2} \mathrm{~s}$

temperature increased linearly, this time to about $165^{\circ} \mathrm{F}\left(74^{\circ} \mathrm{C}\right)$. The temperature difference approached $140^{\circ} \mathrm{F}\left(60^{\circ} \mathrm{C}\right)$.

The third test was conducted at $100 \mathrm{Btu} / \mathrm{ft}^{2} \mathrm{~s}$. The results are shown in Figure 104. The heat flux exposure period for this test began at about 6 seconds, and ended at about 12 seconds, for a total exposure period of about 6 seconds. As in the other tests, the surface temperature responded first with a sharp rise in temperature, followed by continued temperature rise at a slower rate. The maximum surface temperature was about $400^{\circ} \mathrm{F}\left(204^{\circ} \mathrm{C}\right)$. The backside temperature again rose linearly. The maximum backside temperature reached was $235^{\circ} \mathrm{F}\left(113^{\circ} \mathrm{C}\right)$. The temperature difference for this test approached a maximum of just over $200^{\circ} \mathrm{F}\left(93^{\circ} \mathrm{C}\right)$.

The data from these tests was sent to D.G. Walker. The data reduction method described earlier would be used at a later date to calculate the heat flux estimate. The primary goal of these tests, however, was to verify that the heat flux sensor would provide good temperature histories. These experiments also provided a chance to test the method of acquiring the Type $\mathrm{T}$ thermocouples in series as a millivolt signal, using the Type $\mathrm{K}$ thermocouple as a reference, and post-processing to get the surface temperature. 


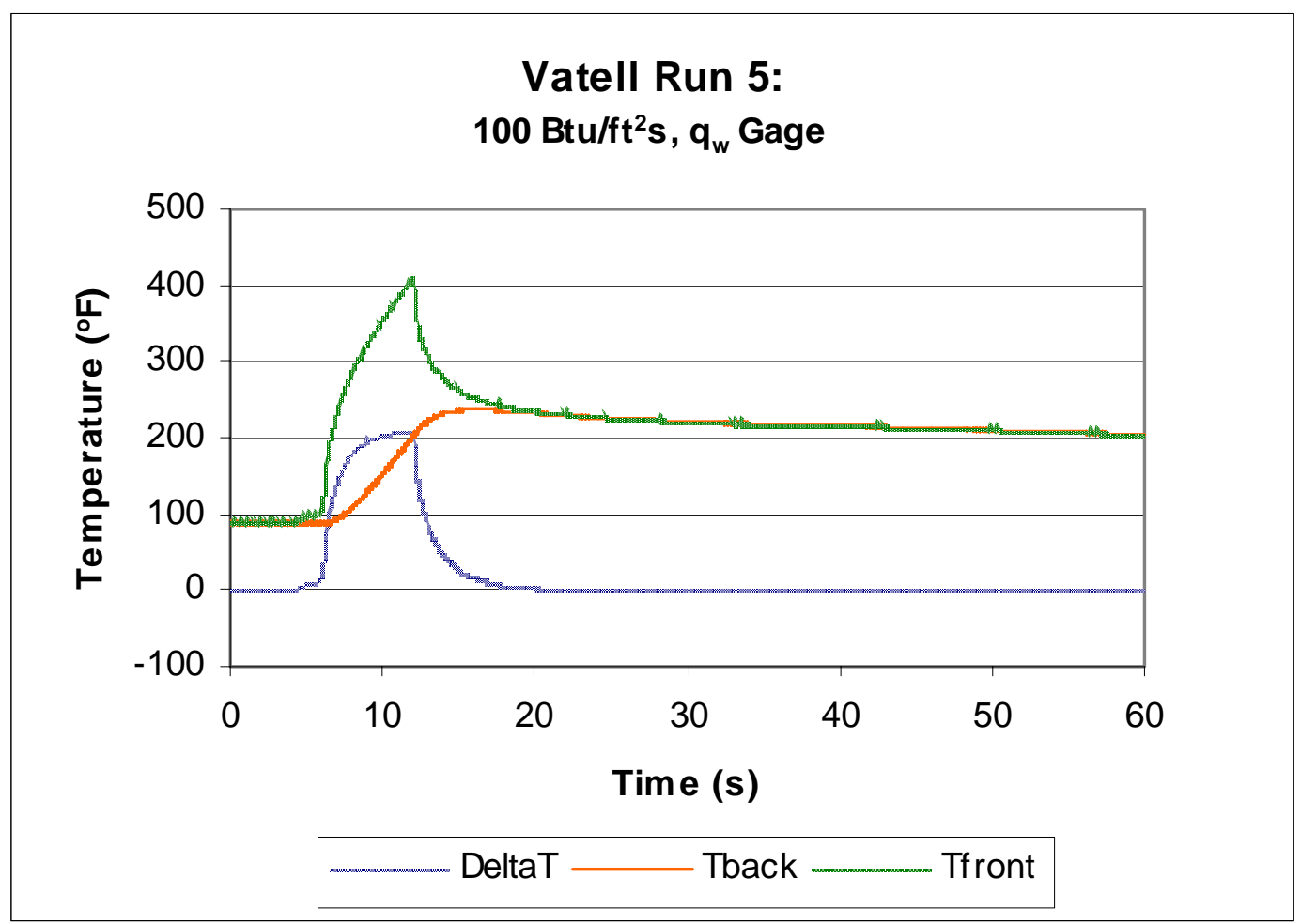

Figure 104: Heat Load Test Results: 100 Btu/ft2s

This post-processing method was successful in providing the surface temperature histories seen above. The temperature histories from these tests made good sense, and had little noise.

After the heat load tests the heat flux gage was visually inspected. The gage showed no visual sign of damage. These tests showed that the heat flux gage was functioning as designed and could operate in high heat flux environments.

\subsection{HXEM Tests}

The heat load tests proved that the heat flux sensor could accurately measure temperature histories in a radiant heat environment. The next step was to verify that the heat flux gage was capable of measurement in a scramjet flow environment. Therefore, before installation on the Hyper-X Mach 10 flight vehicle, it was tested in a wind tunnel ground test of the Hyper-X Engine Model (HXEM). This was the same full-scale partialwidth model of the Hyper-X engine on which the second and third skin friction gages were tested. The heat flux gage was tested at the same time as the third skin friction gage, during the same set of wind tunnel tests. 


\subsubsection{Test Facility}

The facility used for these tests was the NASA Langley Arc-Heated Scramjet Test Facility (AHSTF). This facility was described in detail in Section 5.4.1. The wind tunnel and the engine model (HXEM) were the same as for the previous tests. The reader is referred to the previous discussion for a description of the wind tunnel operating capabilities and procedures.

The same wiring configuration as shown in Figure 51 was used for the heat flux gage. A 2310 signal-conditioning amplifier was used to acquire the millivolt signal from the two Type $\mathrm{T}$ thermocouples wired in series. Though the 2310 was designed for use with strain gage systems, it can be used to process any signal. The excitation was simply turned off, and the two leads from the heat flux gage were routed to the signal input channels of the 2310 connector. The 2310 amplified the signal from the heat flux gage by a gain factor of 500 . The signal from the 2310 was acquired by the facility NEFF system, as was the signal from the backside Type $\mathrm{K}$ thermocouple. Both signals were sampled at $50 \mathrm{~Hz}$. The Type $\mathrm{K}$ thermocouple signal was filtered at $1 \mathrm{~Hz}$, and the Type $\mathrm{T}$ thermocouples wired in series were filtered at $10 \mathrm{~Hz}$. The facility equipment, rather than the 2310 , performed the filtering of the signal from the heat flux sensor.

The gage was mounted on the inlet ramp of the engine, as was done with the skin friction gage. Again, this was because there was no internal room for the sensors to be mounted in the combustor of the engine. However, the heat flux gage was mounted in different portion of the inlet ramp than the skin friction gage, where the local conditions (Mach number, pressure, temperature) were different.

The earlier tests $\left(2^{\text {nd }}\right.$ skin friction design) in the AHSTF were conducted to simulate the enthalpy of Mach 7 flight. For these tests, however, NASA's test program called for Mach 6.5 flight enthalpy. The nozzle area ratio was the same as before, producing a Mach 6.0 freejet at the nozzle exit. But the nominal total pressure was raised from $460 \mathrm{psia}(3172 \mathrm{kPa})$ for the previous tests to $555 \mathrm{psia}(3827 \mathrm{kPa})$ for these tests. Likewise the total enthalpy was reduced from $1055 \mathrm{Btu} / \mathrm{lb}$ for the previous tests to 900 $\mathrm{Btu} / \mathrm{l} \mathrm{b}_{\mathrm{m}}$ for these tests.

The tunnel operation sequence was as follows. The main and bypass air were opened and allowed to stabilize for a couple of seconds. Then the arc power was turned 
on for a total of about 30 seconds of hot, high enthalpy flow. The bypass air was closed off 5 seconds after the arc power was off, with the main air following about 3.5 seconds later. The tunnel condition for the shutdown sequence is unknown. With the arc off and the bypass air off, the flow is due to the main air alone. The flow from the nozzle exit may or may not be supersonic, and without heating it is likely to be very cold with possible condensation of the air.

\subsubsection{Test Results}

A total of six high-enthalpy runs were performed with the heat flux gage design in the HXEM. The results of these tests are shown in Figure 105 through Figure 110. Table 6 summarizes the numerical output, and Table 7 gives results of different heat flux estimation methods based on those results. In addition there were two runs in which the arc power did not come on. Without the arc, the flow in the tunnel was at a low enthalpy, and no heating occurred, so the results of these are not shown. The first run was a trial run in which the arc was only powered for about five seconds. All the other runs followed the run procedure noted above, with the arc on for a total of about 30 seconds. This test procedure results in a relatively long exposure to the high-enthalpy flow. The flight test, in comparison, is only planned to expose the engine to 7 to 15 seconds of hot flow.

The output from the heat flux sensor was extremely repeatable for all of the runs. The output clearly followed the tunnel events, with fast response in surface temperature as the arc came on. For runs that were conducted shortly after the previous run, the temperature of the gage at the start of the consecutive run was higher than at the start of the previous run. This can be seen in the temperature history of the gage shown in Figure 111. This indicated that the temperature of the engine continued to rise for a time after shutdown as the heat soaked into the engine walls.

In Figure 105 through Figure 110, the surface temperature, the backside temperature, and the temperature difference are shown for each run, along with the total pressure. It is clear when comparing the charts that the heat flux gage gave extremely similar results in each run. The charts show consistently steady temperature differences of about $13.1^{\circ} \mathrm{F}\left(7.3^{\circ} \mathrm{C}\right)$ during the hot flow test time. In every run there was interference in the surface temperature signal between about 8.5 and 10 seconds, and again between 
about 34 and 36 seconds. The cowl actuation motor that opened and closed the engine cowl at the beginning and end of each hot test period caused this. This was quite a large motor, and it produced electromagnetic interference during operation.

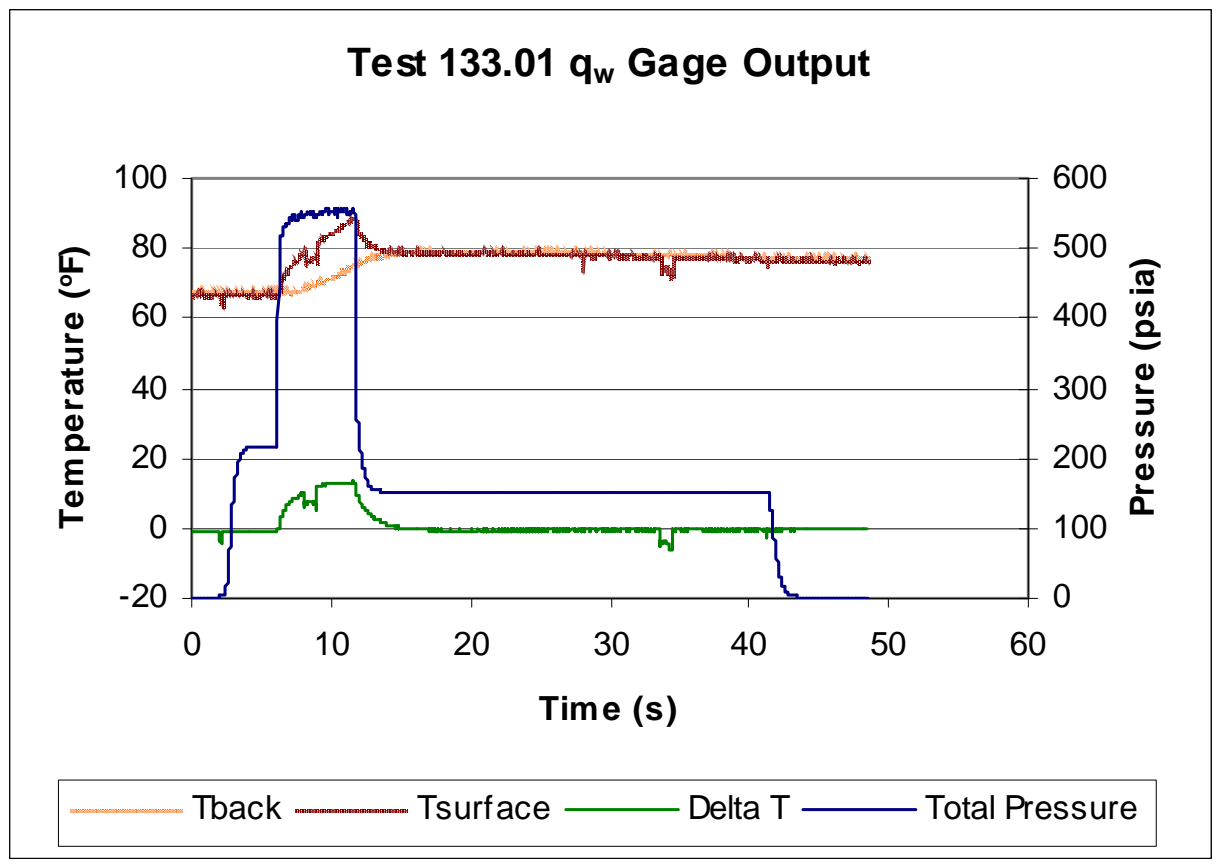

Figure 105: AHSTF Test Results: Run 133.01

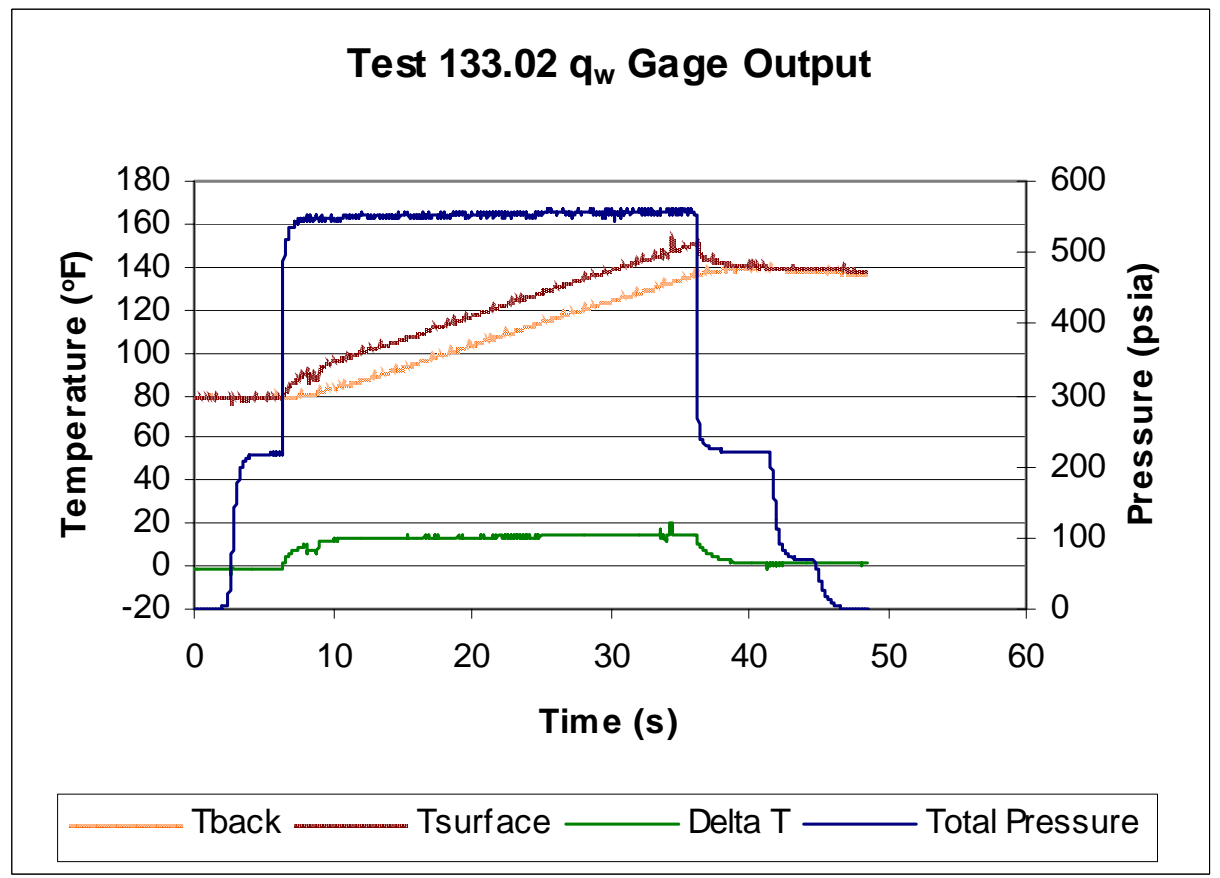

Figure 106: AHSTF Test Results: Run 133.02 


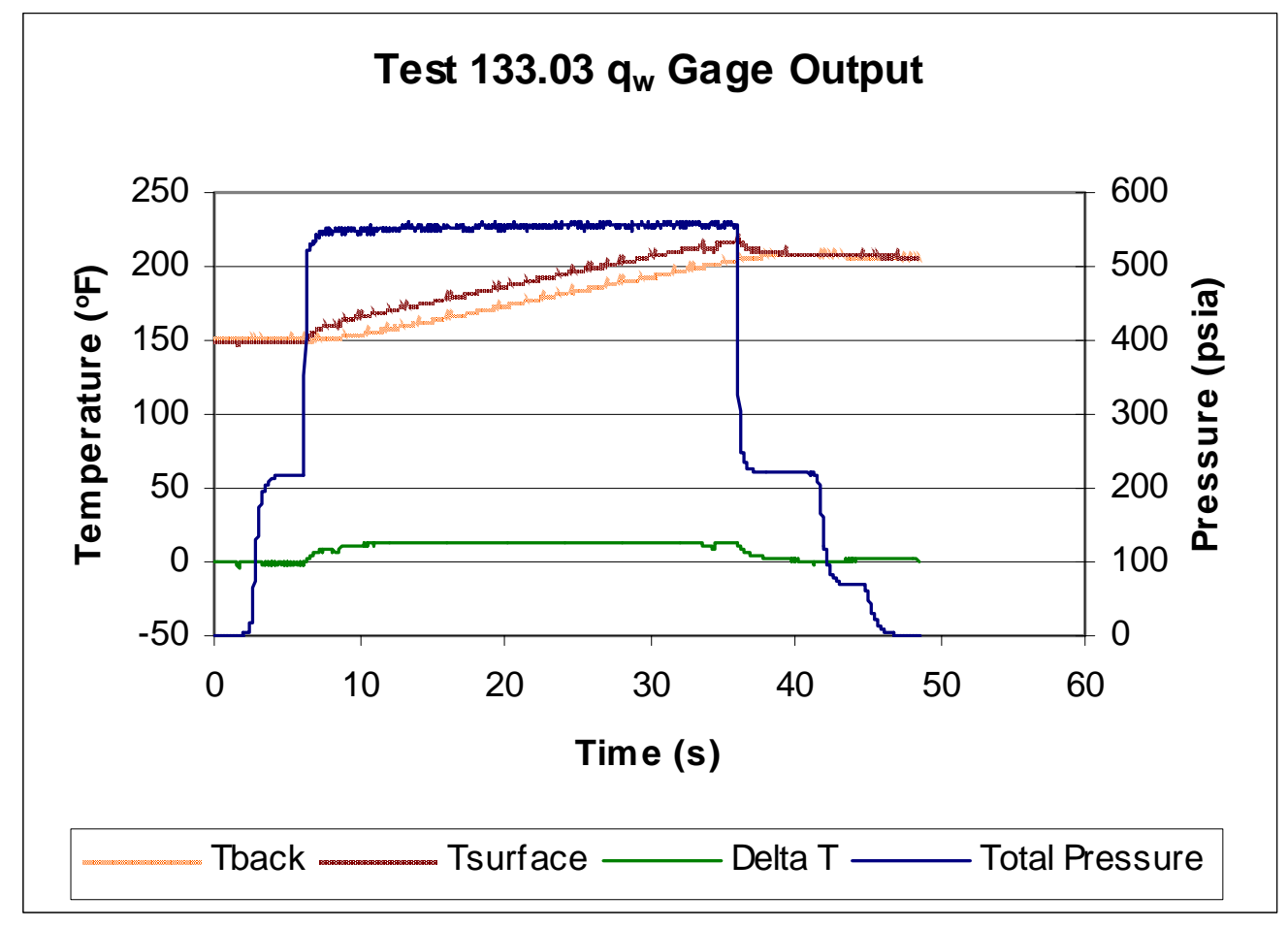

Figure 107: AHSTF Test Results: Run 133.03

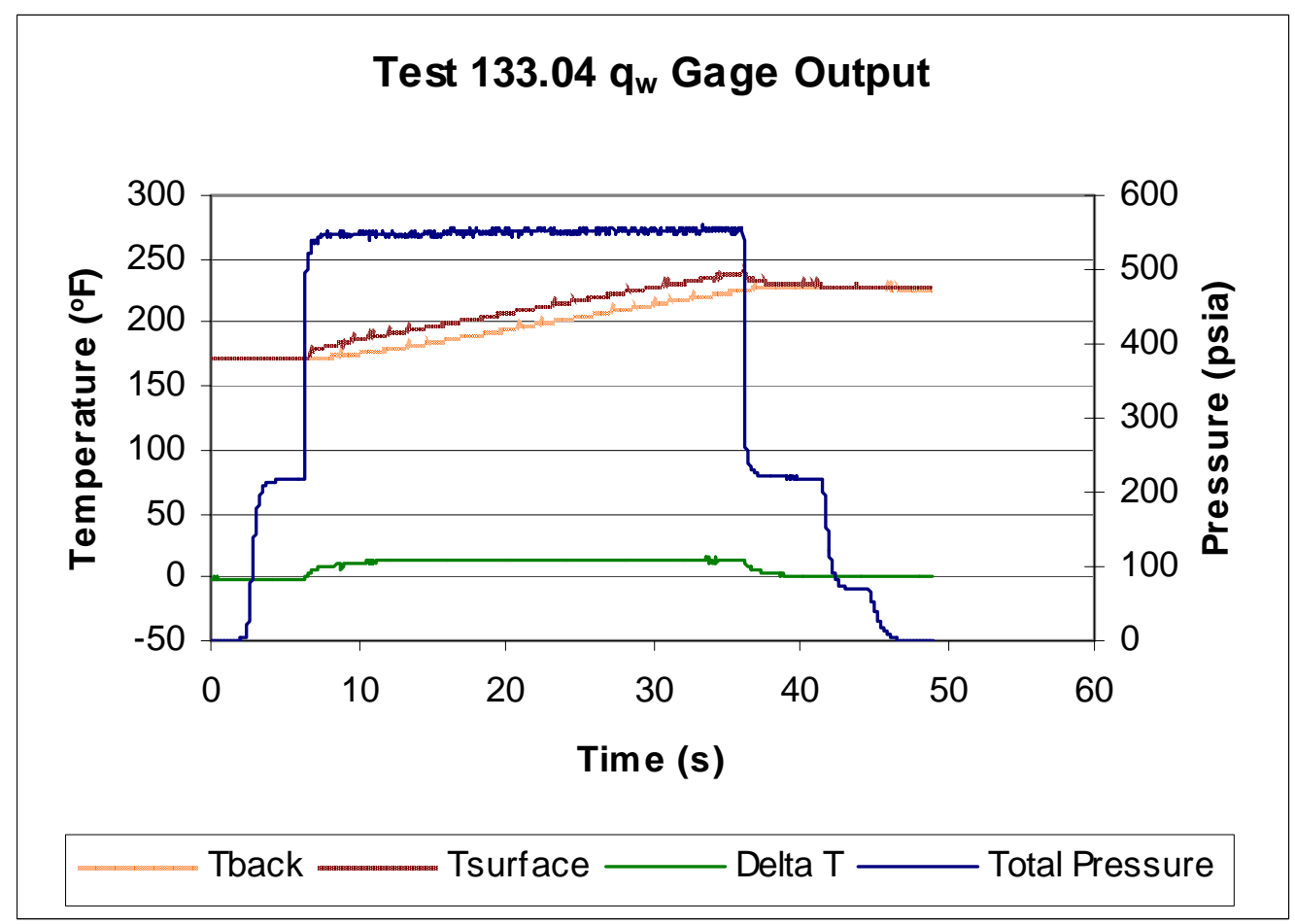

Figure 108: AHSTF Test Results: Run 133.04 


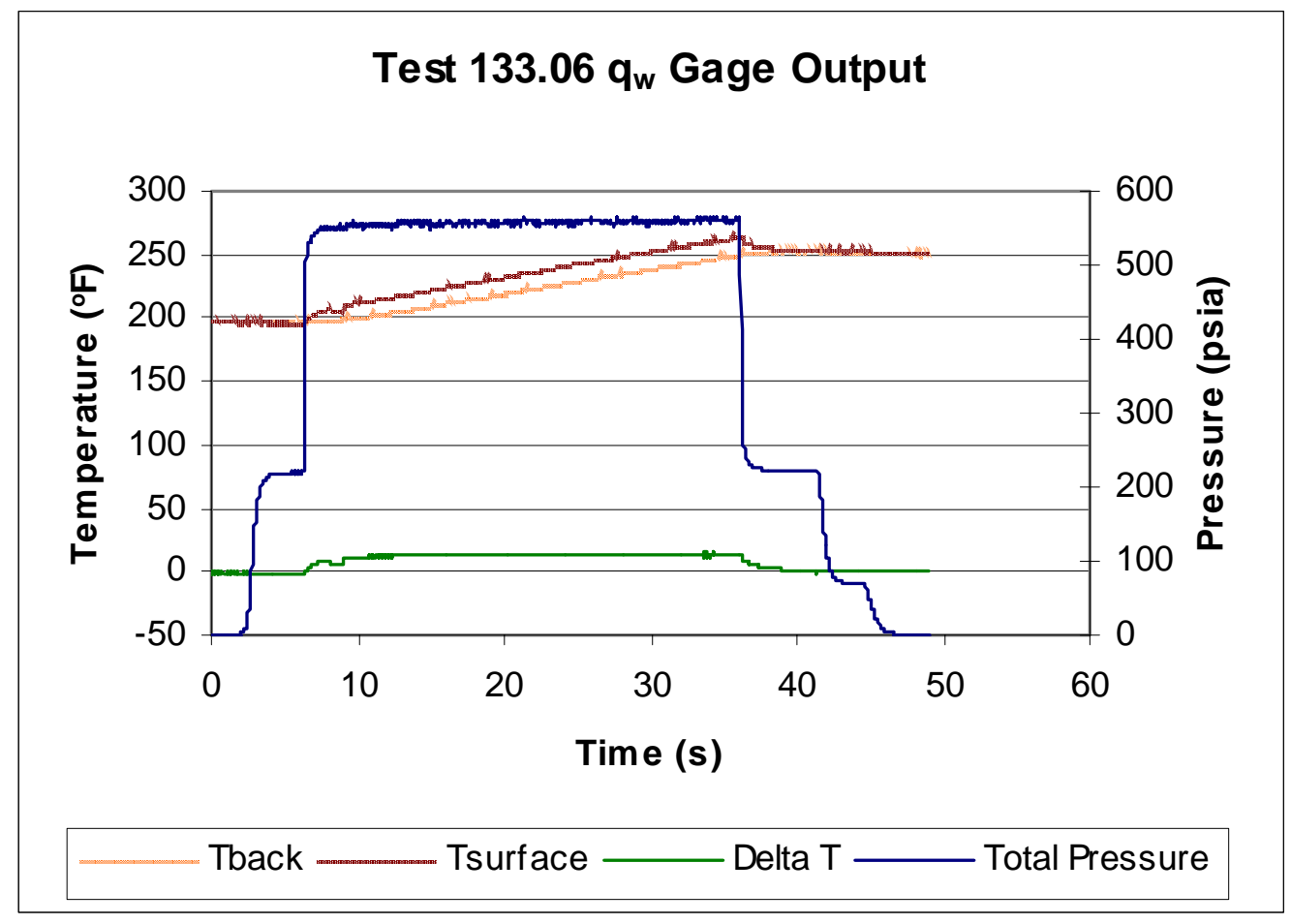

Figure 109: AHSTF Test Results: Run 133.06

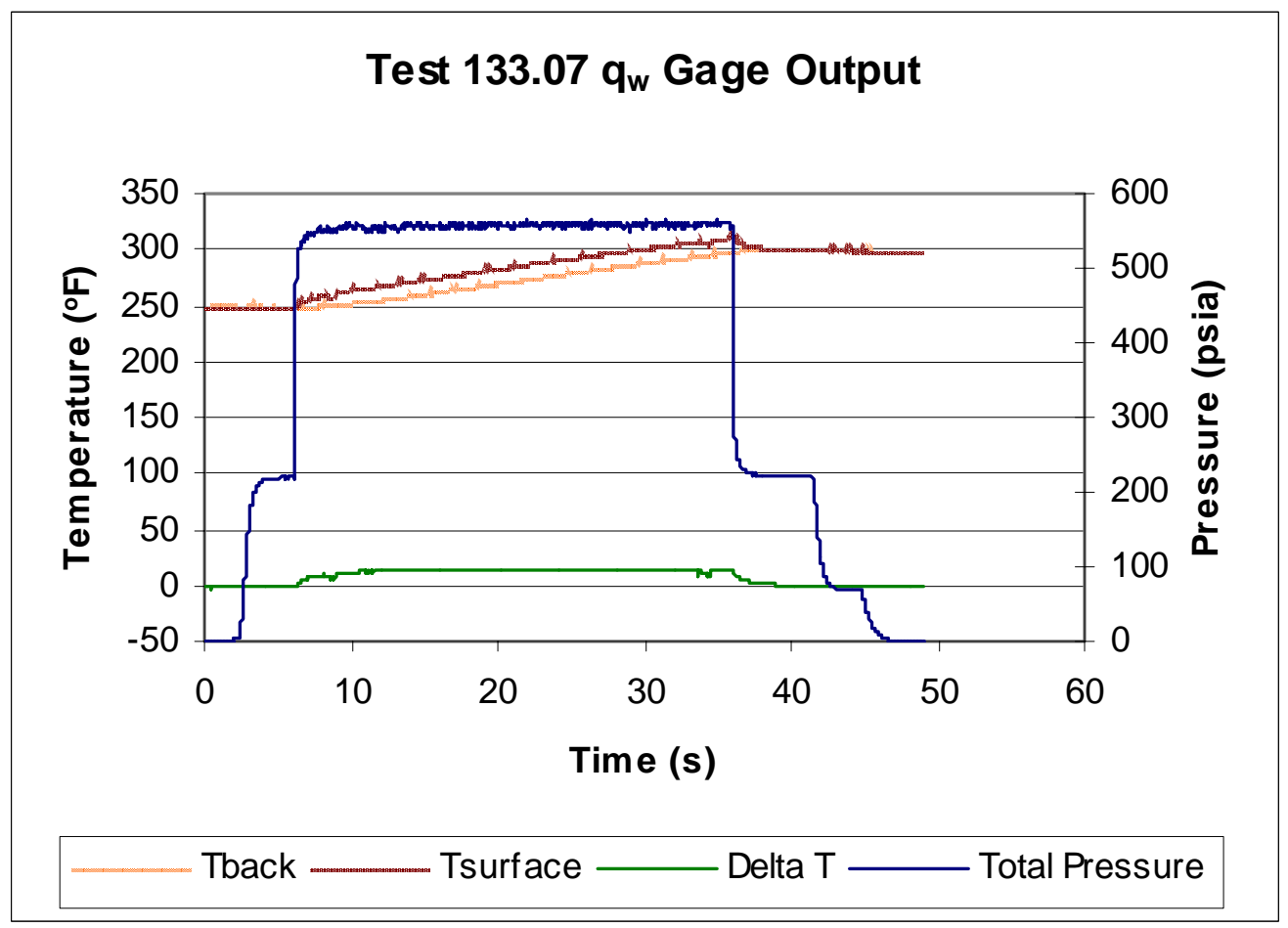

Figure 110: AHSTF Test Results: Run 133.07 


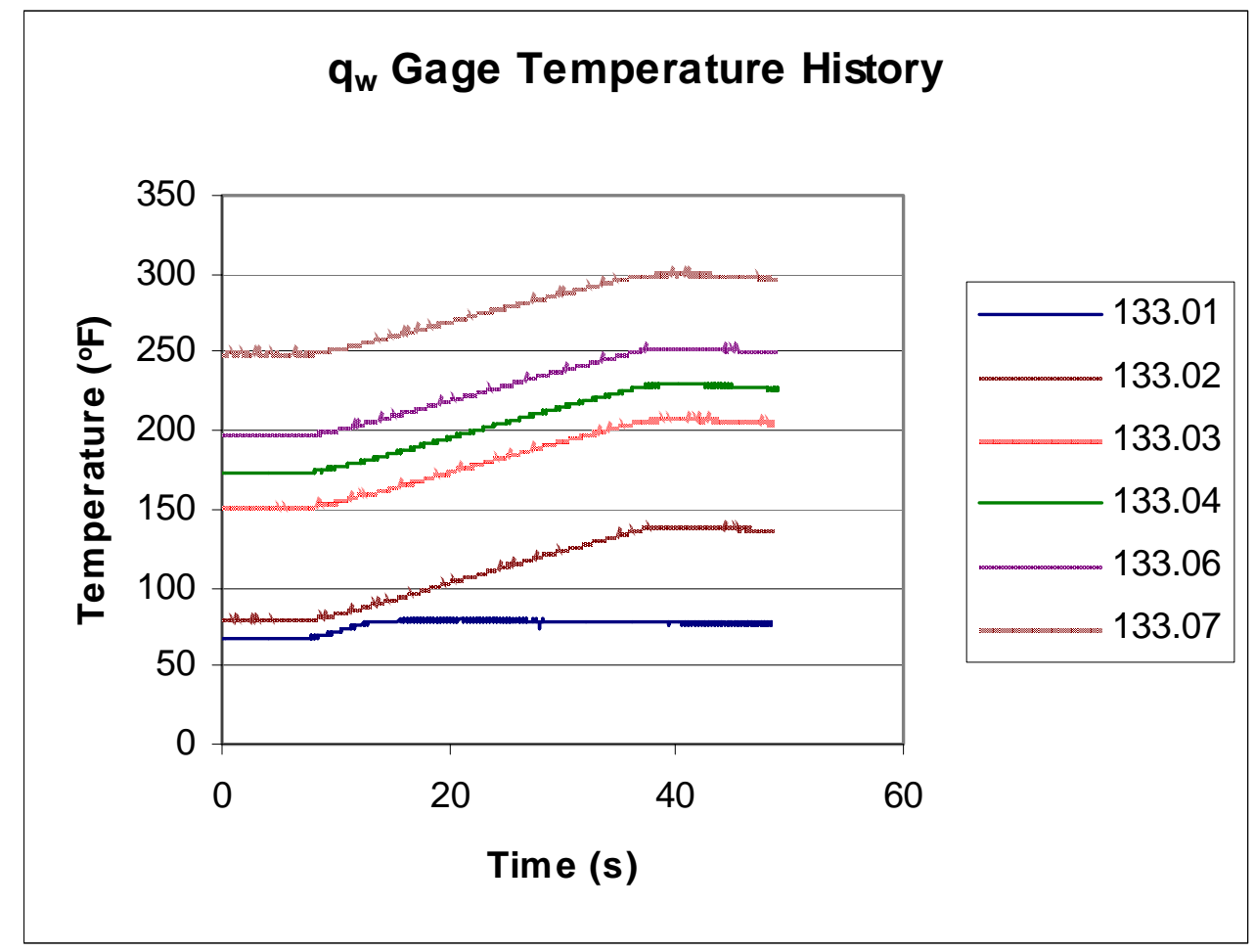

Figure 111: AHSTF Test Results: Gage Backside Temperature History

\begin{tabular}{|c|c|c|c|}
\cline { 2 - 4 } \multicolumn{1}{c|}{} & \multicolumn{3}{c|}{ Measurements } \\
\hline \multirow{2}{*}{ Run } & \multicolumn{2}{c|}{$\mathrm{T}_{\max }\left({ }^{\mathrm{O}} \mathrm{F}\right)$} & Average \\
\cline { 2 - 4 } & Surface & Backside & $\Delta \mathrm{T}\left({ }^{\circ} \mathrm{F}\right)$ \\
\hline 133.01 & 88 & 79 & 13.1 \\
133.02 & 150 & 139 & 13.7 \\
133.03 & 218 & 208 & 13.1 \\
133.04 & 240 & 229 & 13.1 \\
133.06 & 264 & 253 & 13.2 \\
133.07 & 311 & 300 & 13.0 \\
\hline
\end{tabular}

Table 6: AHSTF Test Results Summary

The results are summarized in Table 6, which also shows the maximum temperatures for each run. As seen in Figures 105-110, the temperature difference reached a nearly constant value during each run. This makes sense because the copper material of the sensor conducts heat so well. This indicated that the heat transfer through the gage reached a steady equilibrium state. For this case, an estimate of the heat flux was made assuming the one-dimensional steady heat conduction equation, shown below: 


$$
q_{w}=-k\left(\frac{\partial T}{\partial y}\right) \approx-k\left(\frac{\Delta T}{\Delta y}\right)
$$

\section{Equation 24}

The thermal conductivity, $\mathrm{k}$, was taken as that of the copper plug, and the vertical distance between the surface and backside thermocouples was used for $\Delta y$. The data from these tests was also post-processed by D.G. Walker using the Class 3 Method discussed earlier. Figure 112 through Figure 117 show the results of this post-processing for the hot tests in the AHSTF. These figures show the heat flux estimate, the resulting surface temperature history calculated based on the physical properties of the sensor, and the measured surface temperature history. The estimated surface temperature histories match the measured surface temperature histories. The electromagnetic interference due to the cowl actuator motor is seen in the thermocouple signal, and is reproduced in the heat flux estimates. In addition to Walker's estimates, NASA performed a CFD solution of the AHSTF/HXEM flow. The methodology is discussed in Appendix C.4.

The results of the different heat flux estimation methods are shown for comparison in Table 7. The negative values of the heat flux indicate heat being transferred out of the fluid (and into the wall). There was pretty good agreement between the three methods. The 1-D method predicted heat fluxes of $-10.8 \mathrm{Btu} / \mathrm{ft}^{2} \mathrm{~s}$. The numerical post-processing indicated average fluxes of $-9.1 \mathrm{Btu} / \mathrm{ft}^{2} \mathrm{~s}$, with maximum values of about $-9.6 \mathrm{Btu} / \mathrm{ft}^{2} \mathrm{~s}$ during later periods in some of the tests. The CFD solution predicted the heat flux as $-9.77 \mathrm{Btu} / \mathrm{ft}^{2} \mathrm{~s}$. The 1-D method likely over-predicts the heat flux because of material properties and lateral conduction in the actual case ${ }^{56}$. These tests allowed a good check on the Class 3 method, since the estimate from the numerical algorithm was compared to the one-dimensional calculation and the CFD prediction.

\begin{tabular}{|c|c|c|c|c|c|c|}
\cline { 2 - 7 } \multicolumn{1}{c|}{} & \multicolumn{2}{c|}{ 1-D Estimate } & \multicolumn{2}{c|}{ Class 3 Estimate } & \multicolumn{2}{c|}{ CFD Estimate } \\
\hline \multirow{3}{*}{ Run } & \multicolumn{2}{c|}{ Average $\mathrm{q}_{\mathrm{w}}$} & \multicolumn{2}{c|}{ Average $\mathrm{q}_{\mathrm{w}}$} & \multicolumn{2}{c|}{ Average $\mathrm{q}_{\mathrm{w}}$} \\
\cline { 2 - 7 } & $\left(\mathrm{Btu} / \mathrm{ft}^{2} \mathrm{~s}\right)$ & $\left(\mathrm{W} / \mathrm{m}^{2}\right)$ & $\left(\mathrm{Btu} / \mathrm{ft}^{2} \mathrm{~s}\right)$ & $\left(\mathrm{W} / \mathrm{m}^{2}\right)$ & $\left(\mathrm{Btu} / \mathrm{ft}^{2} \mathrm{~s}\right)$ & $\left(\mathrm{W} / \mathrm{m}^{2}\right)$ \\
\hline 133.01 & -10.8 & -123100.3 & -9.16 & -104000 & $\mathrm{~N} / \mathrm{A}$ & $\mathrm{N} / \mathrm{A}$ \\
133.02 & -11.3 & -128738.5 & -8.79 & -99800 & $\mathrm{~N} / \mathrm{A}$ & $\mathrm{N} / \mathrm{A}$ \\
133.03 & -10.8 & -123100.3 & -9.13 & -103700 & $\mathrm{~N} / \mathrm{A}$ & $\mathrm{N} / \mathrm{A}$ \\
133.04 & -10.8 & -123100.3 & -9.10 & -103400 & $\mathrm{~N} / \mathrm{A}$ & $\mathrm{N} / \mathrm{A}$ \\
133.06 & -10.9 & -124040 & -9.17 & -104100 & $\mathrm{~N} / \mathrm{A}$ & $\mathrm{N} / \mathrm{A}$ \\
133.07 & -10.8 & -122160.6 & -8.83 & -100300 & -9.77 & -111000 \\
\hline
\end{tabular}

Table 7: AHSTF Test Heat Flux Estimates 


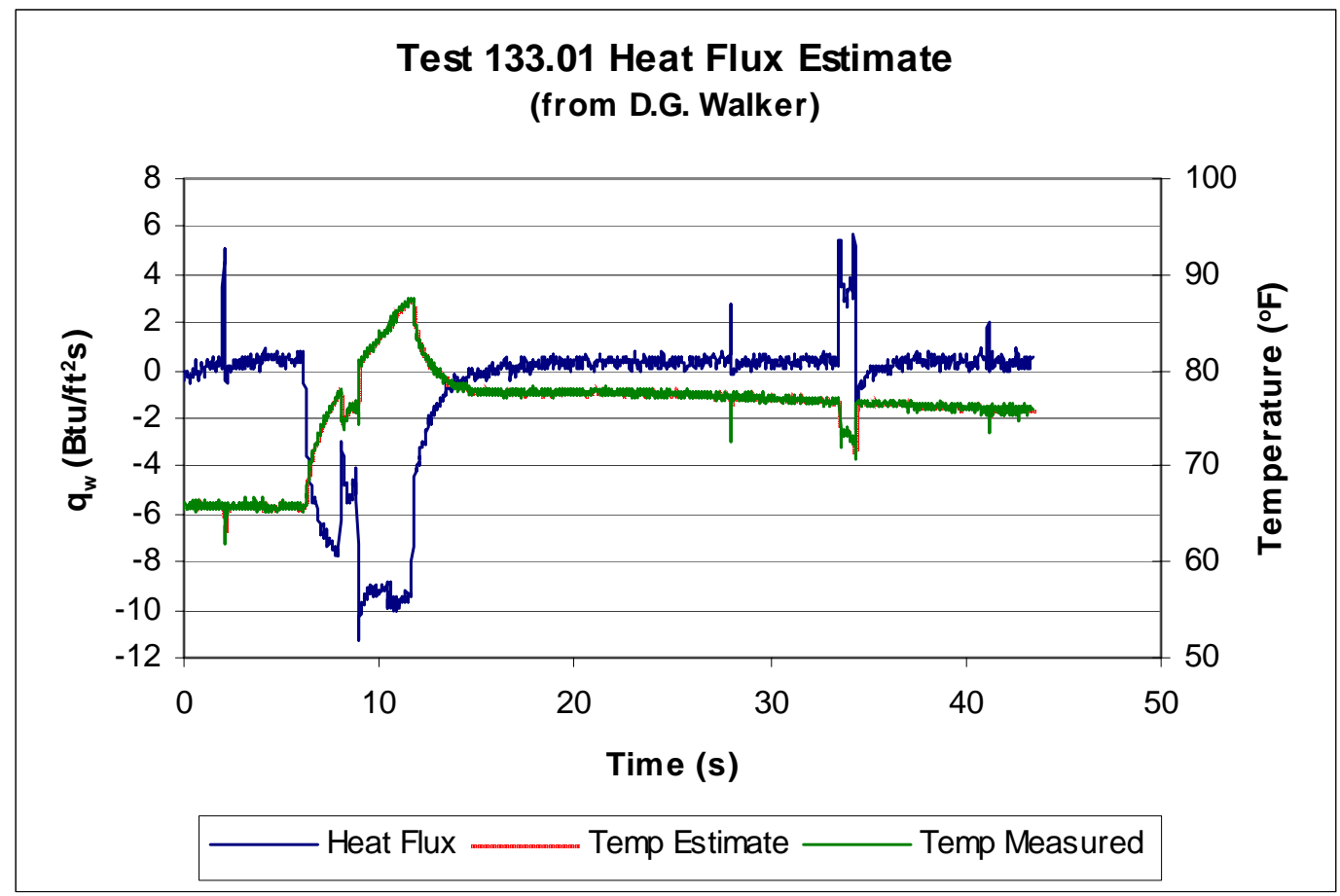

Figure 112: AHSTF Heat Flux Estimate (Class 3): Run 133.01

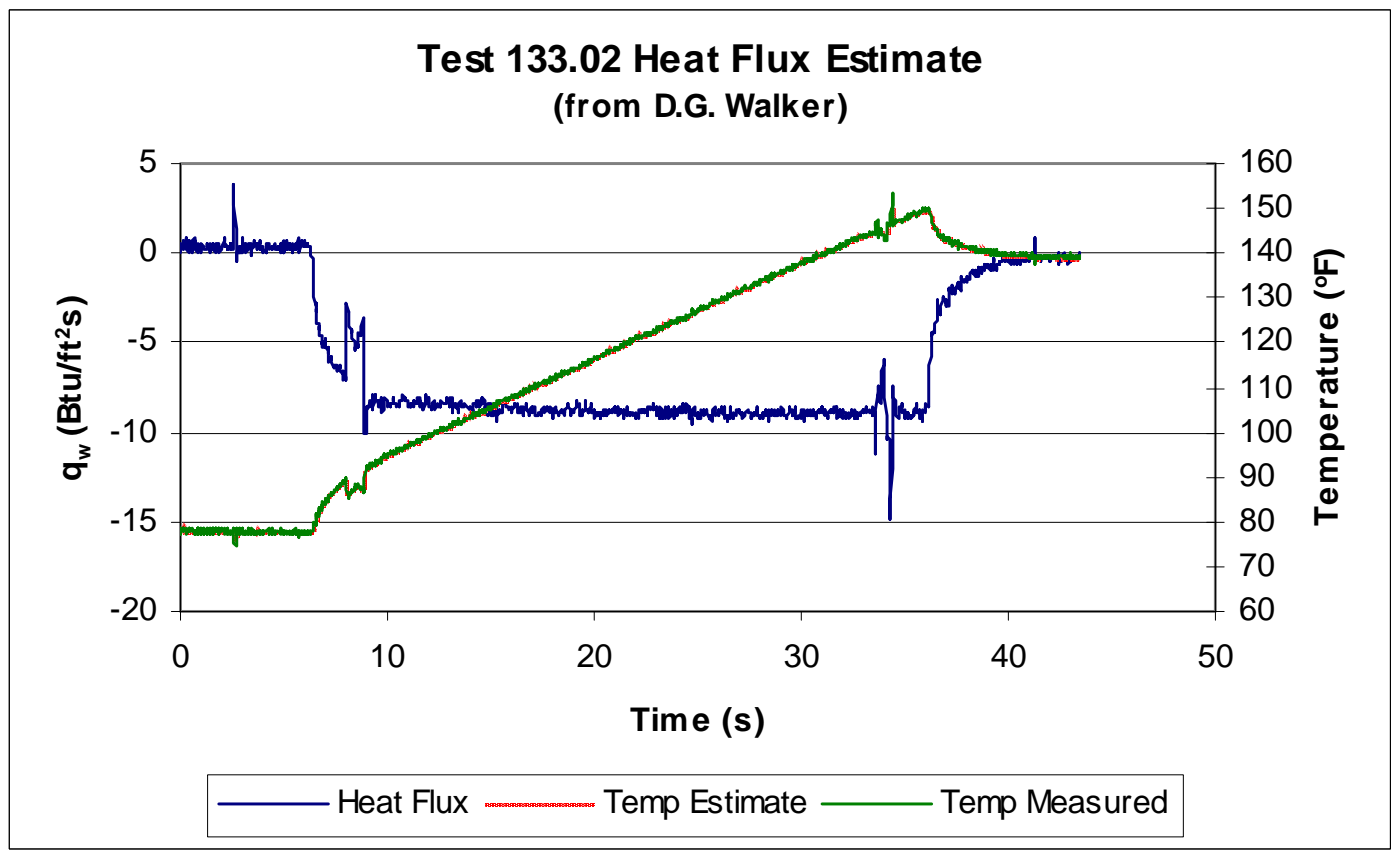

Figure 113: AHSTF Heat Flux Estimate (Class 3): Run 133.02 


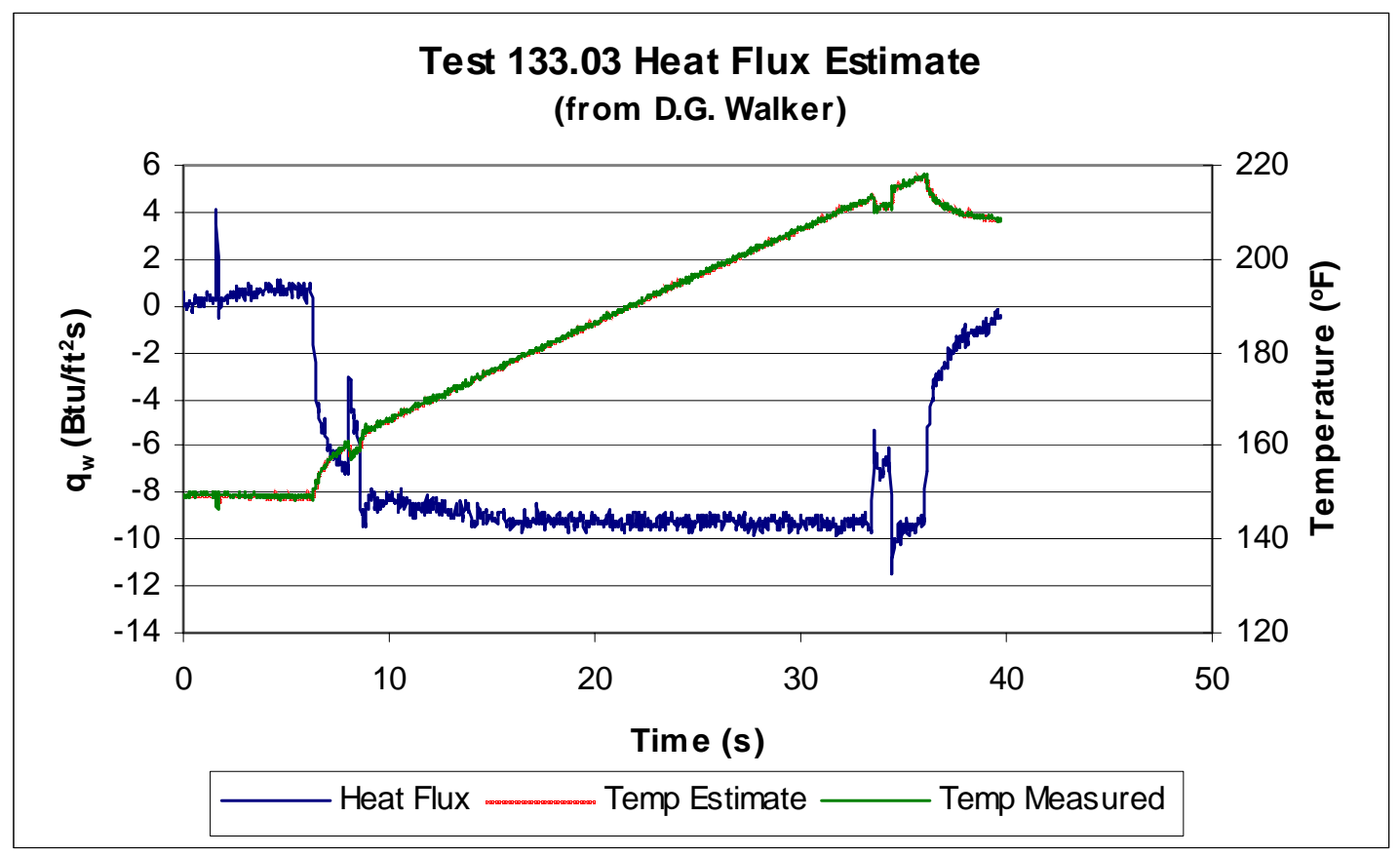

Figure 114: AHSTF Heat Flux Estimate (Class 3): Run 133.03

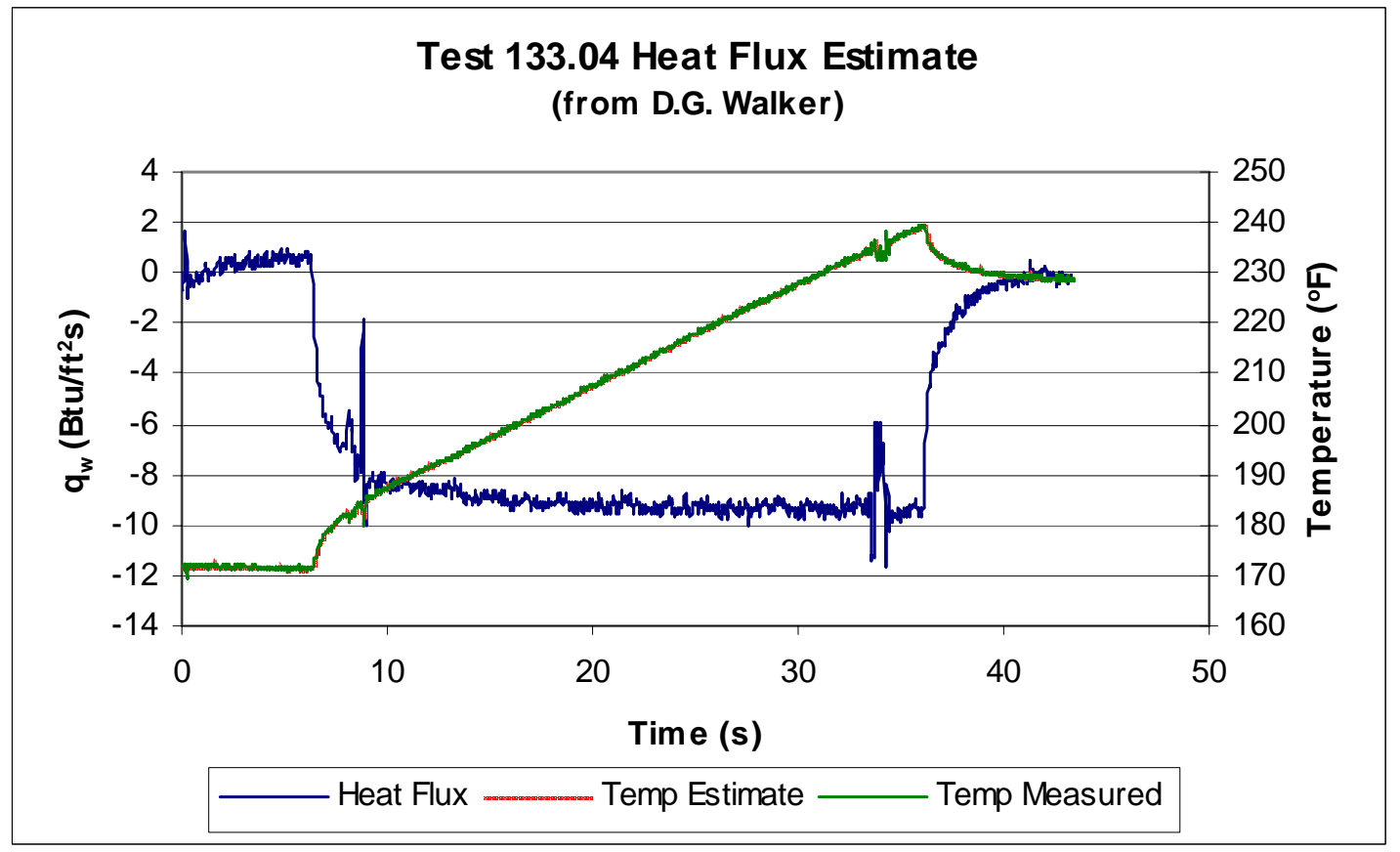

Figure 115: AHSTF Heat Flux Estimate (Class 3): Run 133.04 


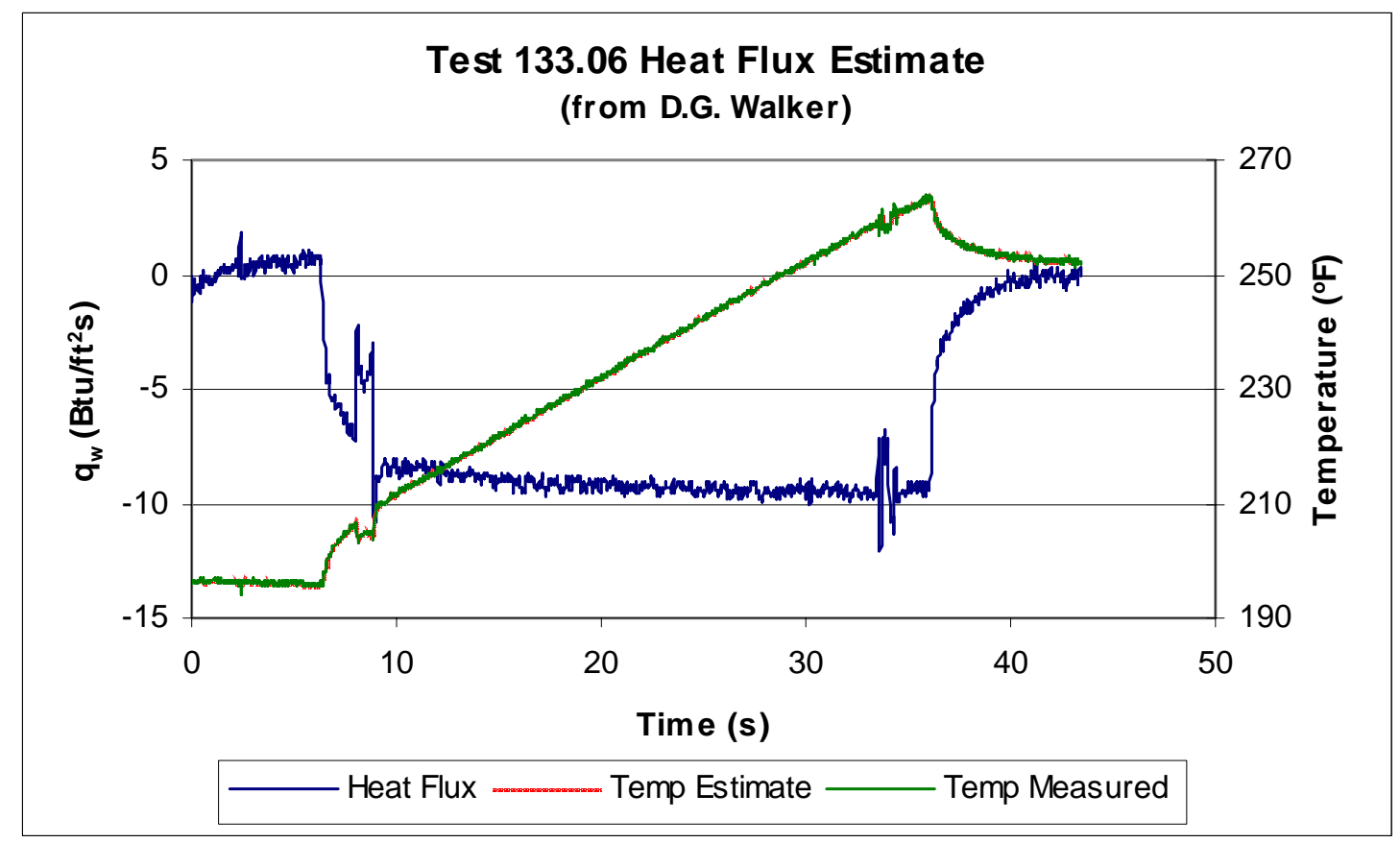

Figure 116: AHSTF Heat Flux Estimate (Class 3): Run 133.06

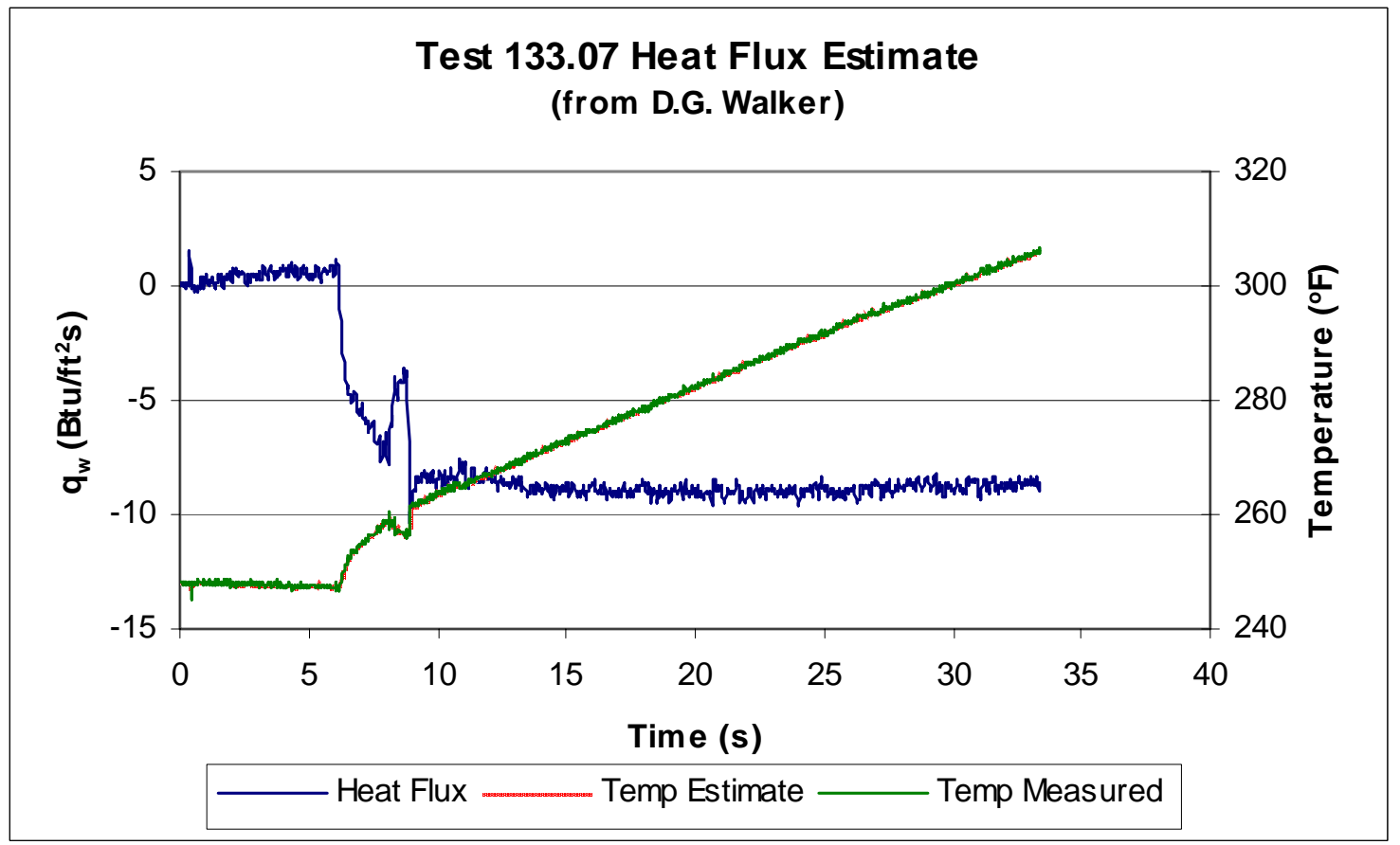

Figure 117: AHSTF Heat Flux Estimate (Class 3): Run 133.07 
One of the goals of developing the heat flux gage was to investigate the Reynolds Analogy for scramjet engine flows. The heat flux and skin friction gages were in different locations of the inlet during the HXEM tests. This, unfortunately, meant that the Reynolds Analogy could not be calculated for these tests. The measurements planned for the Hyper-X flight test, though, should allow for the Reynolds Analogy to be studied.

A visual inspection of the heat flux gage after the AHSTF tests again showed no sign of damage to the gage.

\subsection{Concluding Remarks About The Heat Flux Gage}

The tests in the Hyper-X Engine Model were the conclusion of the heat flux sensor development. The tests in the HXEM provided the closest simulation of the flight vehicle environment possible on the ground. In these tests it was shown that the simple configuration of the heat flux sensor would provide good time histories of surface and backside temperatures in a scramjet engine environment. These time histories can then be used to determine the heat flux into the engine walls of the Hyper-X flight vehicle.

Not only were the gage configuration and operating principle confirmed. The heat flux sensor was also proved to be flight worthy by being passed through the environmental qualification tests. Appendix C presents the NASA reports that officially document the qualification effort. The simple, solid construction of the sensor makes it even more robust than the skin friction sensor, which is more massive and includes the sensitive flexure ring. For this reason it was proposed that heat flux sensors take the place of skin friction sensors in the flight test, in the event that the skin friction sensor should not be available or flight worthy. However, as was discussed in the previous chapter, the skin friction gage should be available and capable of testing on the Hyper-X flight vehicle. In that case, the heat flux gage will be incorporated such that a comparison of the skin friction and heat flux in the engine can be made. The Reynolds Analogy can then be evaluated in the engine of a scramjet during flight. 


\section{CHAPTER EIGHT:}

\section{CONCLUSIONS AND RECOMMENDATIONS}

\subsection{Conclusions}

The major goal of this research was to produce a direct-measuring skin friction gage to be installed in the scramjet engine of the third Hyper-X Research Vehicle (HXRV), designated X-43A. Three different skin friction sensor designs were produced to meet the goal of accurately measuring the wall shear in such a high-enthalpy, supersonic flow.

The first two of these sensors followed in the path of earlier scramjet-tested skin friction sensors from Virginia Tech. They incorporated semiconductor strain gages on a cantilever beam, had active water-cooling systems, and used silicon oil to fill their interior volumes. The first gage was successfully tested in both the Virginia Tech 9-inch by 9-inch supersonic wind tunnel and in a wind tunnel model of a Rocket-BasedCombined-Cycle scramjet combustor. The former test was a Mach 2.4 cold flow test, while the latter test was conducted at Mach 6.4 in the GASL Leg IV freejet facility with full flight enthalpy simulation $\left(\mathrm{P}_{0}=1350 \mathrm{psia}, \mathrm{T}_{0}=2800^{\circ} \mathrm{R}\right)$. The second gage, however, revealed some of the difficulties of incorporating the active cooling systems and oil-fill into a gage to be tested on a scramjet flight vehicle.

Therefore, a radically different design was developed. The third skin friction gage used foil strain gages on a very different flexible member, had no active cooling, and had no oil-fill. Foil strain gages were used due to their relative insensitivity to temperature compared to semiconductor strain gages. The new flexible member was in the form of a ring with very thin spokes. Materials were chosen to provide passive thermal isolation of the strain gages. High temperature gaskets were included to mitigate the risk of hot gas burn through so that the gage, if it failed during flight, would not pose a threat to the rest of the flight vehicle. This third gage also incorporated a flight-worthy electrical connector.

The third skin friction gage was thoroughly tested in many different facilities to 
prove its flight-worthiness. The gage was tested in pressure/altitude and temperature chambers to simulate pressures and temperatures from sea level in the desert $\left(160^{\circ} \mathrm{F}\right.$ to 100,000 feet and $-40^{\circ} \mathrm{F}$. It was shaken at $8 \mathrm{~g}_{\mathrm{rms}}$ from 15 to $2000 \mathrm{~Hz}$ to simulate the flight vibration environment, and it was also shaken to destruction to determine failure modes. The gage was exposed to very high levels (up to $70 \mathrm{Btu} / \mathrm{ft}^{2} \mathrm{~s}$ ) of heat flux such as expected during flight. The skin friction gage passed all of these tests with great success.

More fundamentally important, however, the gage was tested in the Virginia Tech supersonic wind tunnel at Mach 2.4 with $\mathrm{P}_{0}=50$ psia and $\mathrm{T}_{0}=540^{\circ} \mathrm{R}$. These tests proved it could measure the skin friction in a relatively simple flow within about 10-15\% of an estimated value. Finally, as the final pre-flight verification, the third design skin friction gage was tested in a model of the Hyper-X engine in the NASA Langley ArcHeated Scramjet Test Facility. This facility simulated Mach 6.5 flight enthalpy by providing a Mach 6.0 freejet and $\mathrm{P}_{0}=555 \mathrm{psia}$ and $\mathrm{h}_{0}=900 \mathrm{Btu} / \mathrm{l} \mathrm{b}_{\mathrm{m}}$. The successful results of these tests indicated that the skin friction gage developed in this work is ready for installation and testing in the Hyper-X Research Vehicle scramjet engine.

In the development of the final skin friction gage, it became desirable to also design a heat flux sensor for the scramjet flight test. A simple design was developed that incorporated a fast-response coaxial thermocouple mounted flush with the surface of a copper plug. A separate thermocouple was also mounted at a known distance from the surface inside the copper plug. This configuration was designed to provide accurate time histories of the temperature on the surface and at a depth inside the copper plug. An inverse method was then used to calculate an estimate of the heat flux into the copper plug. The heat flux sensor was qualification tested in temperature and pressure chambers. This sensor was also tested in a radiant heat flux facility. Lastly, the heat flux gage was successfully tested in the scramjet engine model. The use of the heat flux gage in the scramjet engine of the flight vehicle at locations near the skin friction sensors will help to extend the Reynolds Analogy to such complicated flowfields. Extending the Reynolds Analogy to such flows would be a very useful prediction tool.

\subsection{Recommendations}

As with any instrumentation development, the greatest task is to design an instrument that can accurately measure the desired parameter with little or no sensitivity 
to other parameters. For a direct measuring skin friction sensor as was developed for this work, the greatest interfering inputs are temperature and pressure. The skin friction sensors of this study were all pre-tested on flat surfaces in supersonic flows, where there is no pressure gradient. In addition, the first two designs used oil-fill to prevent a pressure gradient from acting on the sides of the floating element. Hence pressure gradient effects are not believed to be a major source of error in the tests of the final skin friction gage reported earlier. But, some crude 2-dimensional estimation methods suggest possible errors up to $25 \%$ for the pressure gradient in a scramjet combustor. The actual error would likely be much less due to the three-dimensional relief around the floating head (see Appendix A for Uncertainty Analysis).

In any event, a better understanding of the effects of pressure gradients on skin friction measurements is needed. There is current work being done at Virginia Tech to parametrically quantify the errors of skin friction sensors to pressure gradients. The information from such a study will surely help in the design of skin friction sensors that are insensitive to pressure gradients.

The major interfering input for the skin friction sensors of this work was temperature. This is mostly due to the use of strain gages. And, for tests in high enthalpy supersonic flows, the temperature environment is extreme. For the earlier designs, active cooling systems were incorporated to minimize the possibility of temperature changes. These designs also had oil filling the internal volume, which helped protect the strain gages by providing a physical barrier from the hot flow and a large thermal mass. The final skin friction sensor, however, had neither active cooling nor any oil-fill, and some drift due to temperature was experienced, but it was accounted for without great difficulty.

For future work, however, it would be better to eliminate temperature as an interfering input. The best way to do this would be to use a method of measuring strain that is not temperature sensitive. Fiber-optic technologies have been considered to be promising in this regard, but the technology readiness level is not yet at a stage where fiber-optics can easily be incorporated into an instrument, as can be done with strain gages. In the meantime, there may be ways to make strain-gage-based instruments less temperature sensitive. The simplest way is to design the instrument to produce a larger 
strain so that the temperature portion of the signal makes up only a very small portion of the total signal. A larger floating element and/or a longer moment arm would accomplish this. 


\section{REFERENCES}

\footnotetext{
${ }^{1}$ Sang, A.K., Study of Rubber Damped Skin Friction Gages for Transonic Flight Testing, Master's Thesis, Aerospace and Ocean Engineering Department, Virginia Polytechnic Institute and State University, pp. 1-6, 2001.
}

2 Blasius, H., "Grenzschichten in Fl,ssigkeiten mit kleiner Reibung," [Boundary Layers in Fluids with Small Viscosity], ZAMP, Vol. 56, No. 1, pp. 1-37, 1908. [Available in translation as NACA TM 1256, 1950.]

3 Schetz, J.A., "Direct Measurement of Skin Friction in Complex Fluid Flows," Applied Mechanical Review, vol. 50, no 11, part 2, pp. S198-S203, Nov. 1997.

${ }^{4}$ Schetz, J.A., Boundary Layer Analysis, Prentice Hall, Englewood Cliffs, New Jersey, pp. 206-208, 1993.

5 Moszee, R., and Snyder, C., "A Propulsion Development Strategy for the National Aero-Space Plane," AIAA-89-2751, 25th Joint Propulsion Conference, July 1989.

${ }^{6}$ Billig, F. S., private communication, 1998.

${ }^{7}$ Heiser, W.H., and Pratt, D.T., Hypersonic Airbreathing Propulsion, AIAA Education Series, American Institute of Aeronautics and Astronautics, Washington, DC, pp. 189, 429-434, 1994.

${ }^{8}$ Hazelton, D.M. and Bowersox, R.D.W., "Skin Friction Correlations for High Enthalpy Flows," AIAA-981636, 8th International Space Planes and Hypersonic Systems and Technology Conference, April 1998.

${ }^{9}$ DeTurris, D.J., A Technique for Direct Measurement of Skin Friction in Supersonic Combustion Flow, Ph.D. Dissertation, Aerospace and Ocean Engineering Department, Virginia Polytechnic Institute and State University, pp. 1-16, 1992.

${ }^{10}$ Pulliam, W.J., Development of Fiber Optic Aerodynamic Sensors for High Reynolds Number Supersonic Flows, Ph.D. Dissertation, Aerospace and Ocean Engineering Department, Virginia Polytechnic Institute and State University, pp. 1-24, 2000.

${ }^{11}$ McClinton, C.R., Rausch, D.R., Sitz, J. and Reukauf, P., "Hyper-X Program Status,” AIAA 2001-1910, AIAA/NAL-NASDA-ISAS 10th International Space Planes and Hypersonic Systems and Technologies Conference, April 2001.

12 Erdos, J.I., "On the Bridge from Hypersonic Aeropropulsion Ground Test Data to Flight Performance," AIAA 98-2494, 20th AIAA Advanced Measurement and Ground Testing Technology Conference, June 1998.

13 von Ofenheim, B. (1999), "NASA Langley Research Center, LISAR: Hyper-X," Retrieved July 26, 2001 from the World Wide Web: http://lisar.larc.nasa.gov/LISAR/BROWSE/hyperx.html.

${ }^{14}$ Froude, W., "Experiments on the Surface-friction Experienced by a Plane Moving through Water," 42nd British Association Report, pp. 118-124, 1872.

${ }^{15}$ Winter, K.G., "An Outline of the Techniques Available for the Measurement of Skin Fiction in Turbulent Boundary Layers," Progress in Aerospace Science, Vol. 18, pp. 1-57, 1977.

${ }^{16}$ Nitsche, W., Haberland, C., and Thunker, R., "Comparative Investigations on Friction Drag Measuring Techniques in Experimental Aerodynamics,” ICAS-84-2.4.1, 14th ICAS Congress, pp. 391-403, 1984. 
${ }^{17}$ Dhawan, S., “Direct Measurements of Skin Friction,” NACA Report 1121, 1953.

18 Coles, D., Measurements in the Boundary Layer on a Smooth Flat Plate in Supersonic Flow. II. Instrumentation and Experimental Technique at the Jet Propulsion Laboratory, JPL Report 20-70, 1953.

19 Allen, J.M., "Systematic Study of Error Sources in Supersonic Skin-Friction Balance Measurements," NASA TN D-8291, 1976.

${ }^{20}$ Allen, J.M., "Improved Sensing Element for Skin-Friction Balance Measurements," AIAA Journal, Vol. 18, No. 10, pp. 1342-1345, 1980.

${ }^{21}$ Voisinet, R., “Temperature Step Effect on Direct Measurement of Skin Friction Drag," AIAA 78-0779, 1978.

${ }^{22}$ Roensch, R.L. and Cadwell, J.D., "Direct Measurement of Skin Friction in a High Reynolds Number Supersonic Blow-Down Wind Tunnel,” Douglas Paper 1728, Douglas Aircraft Company.

${ }^{23}$ Schetz, J.A. and Nerney, B., "Turbulent Boundary Layer with Injection and Surface Roughness," AIAA Journal, Vol. 15, No. 9, pp. 1268-1274, 1977.

${ }^{24}$ DeTurris, D.J., Schetz, J.A., and Hellbaum, R.F., "Direct Measurement of Skin Friction in a Scramjet Combustor," AIAA 90-2342, AIAA/SAE/ASME/ASEE 26th Joint Propulsion Conference, July 1990.

${ }^{25}$ Chadwick, K.M., DeTurris, D.J., and Schetz, J.A., "Direct Measurements of Skin Friction in Supersonic Combustion Flowfields,” J. Eng. Gas Turbines and Power, Vol. 115, No. 3, pp. 507-514, 1993.

${ }^{26}$ Bowersox, R. and Schetz, J., "Skin Friction Gages for High Enthalpy Impulsive Flows," AIAA-93-5079, AIAA/DGLR Fifth International Aerospace Planes and Hypersonic Technologies Conference, December 1993.

${ }^{27}$ Novean, M.G., Schetz, J.A., and Bowersox, R.D.W., "Direct Measurements of Skin Friction in Complex Supersonic Flows," AIAA-97-0394, 35th Aerospace Sciences Meeting \& Exhibit, January 1997.

${ }^{28}$ Paros, J.M., “Application of the Force-Balance Principle to Pressure and Skin Friction Sensors," 16th Annual Technical Meeting, Proc. Inst. Environmental Sciences, pp. 363-368, 1970.

${ }^{29}$ Magill, S.A., Study of a Direct Measuring Skin Friction Gage with Rubber Compounds for Damping, Master's Thesis, Aerospace and Ocean Engineering, Virginia Polytechnic Institute and State University, pp. 8-11, 1999.

${ }^{30}$ Lyons, W.C., The Design of an Acceleration Insensitive Skin Friction Balance for use in Free Flight Vehicles at Supersonic Speeds, Master's Thesis, University of Texas, 1957.

31 Fisher, D.F., "Boundary Layer, Skin Friction, and Boattail Pressure Measurements from the YF-12 Airplane at Mach Numbers up to Three," NASA CP 2054, YF-12 Experiments Symposium, Vol. 1, 1978.

32 Quinn, R.D. and Gong, L., "In-Flight Compressible Turbulent Boundary Layer Measurements on a Hollow Cylinder at a Mach Number of 3.0," NASA CP 2054, YF-12 Experiments Symposium, Vol. 1, 1978.

${ }^{33}$ O’Donnell, F.B., and Westkaemper, J.C., "Measurements of Errors Caused by Misalignment of FloatingElement Skin-Friction Balances," AIAA Journal, Vol. 3, No. 1, pp. 163-165, 1965. 
${ }^{34}$ Hakkinen, R.J., "Uncertainties in Measurement of Skin Friction by Conventional and Miniaturized Force-Sensing Elements," American Physical Society Fluid Dynamics Division Meeting, Syracuse, NY, November 1996.

${ }^{35}$ Chadwick, K.M., An Actively Cooled Floating Element Skin Friction Balance for Direct Measurement in High Enthalpy Supersonic Flows, Ph.D. Dissertation, Aerospace \& Ocean Engineering Department, Virginia Polytechnic Institute and State University, pp. 15-26, 1992.

${ }^{36}$ Doebelin, E.O., Measurement Systems, Fourth Edition, McGraw-Hill, Boston, Massachusetts, pp. 220233,1990 .

${ }^{37}$ Lineback, D.L., Editor, "Force and Torque Measurement," Experimental Stress Analysis Notebook, Issue 6, May 1987, Measurements Group, Inc., Raleigh, N.C.

${ }^{38}$ Smith, T.B., Schetz, J.A., and Bui, T.T., "Direct Skin Friction Measurement in a Rocket-BasedCombined-Cycle Scramjet Combustor," AIAA-2000-3724, 36 ${ }^{\text {th }}$ Joint Propulsion Conference, July 2000.

${ }^{39}$ Virginia Tech Aerospace \& Ocean Engineering (1996), "Supersonic Wind Tunnel," Retrieved July 16, 2001 from the World Wide Web: http://www.aoe.vt.edu/aoe/physical/superson.html.

40 GASL, "The Blowdown Laboratory," Retrieved July 16, 2001 from the World Wide Web: http://www.gasl-usa.com/blowdown.htm.

${ }^{41}$ GASL, "Leg IV: A Staged Heating Mach 2 to 8 Free Jet Facility," Retrieved July 16, 2001 from the World Wide Web: http://www.gasl-usa.com/leg4.htm.

${ }^{42}$ Pearson, C.R. (2000), “Arc-Heated Scramjet Test Facility,” Retrieved July 18, 2001 from the World Wide Web: http://wte.larc.nasa.gov/facility/ahstf/ahstf_Brochure/AHSTF_Brochure.karenV1.0.html.

${ }^{43}$ Ruf, E., Site Curator (2001), "Hyper-X Engine Model," Retrieved July 18, 2001 from the World Wide Web: http://hapb-www.larc.nasa.gov/Engines/HXEM/HXEM.html.

${ }^{44}$ Bruno, J.R., Yanta, W.J., and Risher, D.B., "Balance for Measuring Skin Friction in the Presence of Heat Transfer,” NOLTR 69-56, June 1969, U.S. Naval Ordnance Laboratory, White Oak, Maryland.

${ }^{45}$ Goldfeld, M., PetroChenko, V., Nesoulia, R., Shishov, V., and Falempin, F., "The Direct Measurement of Friction in the Boundary Layer at Supersonic Flow Velocities," AIAA-2001-1769, AIAA/NALNASDA-ISAS $10^{\text {th }}$ International Space Planes and Hypersonic Systems and Technologies Conference, Kyoto, Japan, April 2001.

46 “Coaxial Thermocouple Probes," Bulletin 500, Medtherm Corporation, Huntsville, Alabama.

${ }^{47}$ Diller, T.E., "Advances in Heat Flux Measurements," Advances in Heat Transfer, Vol. 23, Eds. J.P. Hartnett et al., Academic Press, Boston, pp. 279-368, 1993.

${ }^{48}$ Bui, T., private communication, July 2001.

${ }^{49}$ Cook, W.J., and Felderman, E.J., "Reduction of data from Thin Film Heat Transfer Gages: A Concise Numerical Technique," AIAA Journal, Vol. 4, No. 3, pp. 561-562,1966.

${ }^{50}$ Cook, W.J., "Determination of Heat Transfer Rates from Transient Surface Temperature Measurements," AIAA Journal, Vol. 8, No. 7, pp. 1366-1368, 1970. 
${ }^{51}$ Cook, W.J., "Unsteady Heat Transfer to a Semi-Infinite Solid with Arbitrary Surface Temperature History and Variable Thermal Properties," TR ISU-ERI-Ames 67500, Engineering Research Institute, Iowa State University, Ames, Iowa, 1970.

${ }^{52}$ Walker, D.G. and Scott, E.P., "Evaluation of Estimation Methods for High Unsteady Heat Fluxes from Surface Measurements," Journal of Thermophysics and Heat Transfer, Vol. 12, p. 543, 1998.

${ }^{53}$ Diller, T.E., "Methods of Determining Heat Flux from Temperature Measurements," Department of Mechanical Engineering, Virginia Tech, Blacksburg, Virginia, pp. 357-369, 1996.

54 Walker, D.G., "Estimation of Unsteady Nonuniform Heating Rates from Surface Temperature Measurements," Ph.D. Dissertation, Virginia Polytechnic Institute and State University, 1997.

${ }^{55}$ Bezuidenhout, J.J., Schetz, J.A., and Walker, D.G., "Heat Flux Determination Using Surface and Backface Temperature Histories and Inverse Methods," AIAA 2001-3530, 37 $7^{\text {th }}$ AIAA/ASME/SAE/ASEE Joint Propulsion Conference \& Exhibit, Salt Lake City, Utah, July 2001.

${ }^{56}$ Walker, D.G., private communication, August 2001. 


\section{APPENDIX A:}

\section{UNCERTAINTY ANALYSIS}

The errors and uncertainty in these direct measurements of skin friction are due to three basic sources. The first of these sources is the uncertainty in the calibration of the sensor and repeatability issues in wind tunnel tests. The second major source is the uncertainty due to thermal drift in the output. Errors caused by the floating head factors such as pressure gradients and misalignment make up the final source.

\section{A.1 Calibration and Repeatability}

The calibrations performed on the skin friction sensors of this study typically provided good results. Good linearity and repeatability of the calibrations were observed for all of the designs. For the final skin friction gage, the maximum difference in output from the same input mass that was observed was $2.8 \%$ of the full-scale output (FSO). A statistical analysis of the all of the calibration data for this skin friction gage produced a standard deviation, s, of $0.80 \%$ FSO. For a Gaussian distribution, 95\% of all samples fall within \pm 2 s of the mean. Therefore, the true load for a given output should be within $1.6 \%$ FSO of the indicated load $95 \%$ of the time.

The final skin friction gage was only calibrated with the static force calibration method. The repeatability, as mentioned above, was good for those tests. The tests of the skin friction gage in dynamic flow environments, however, gave a wider range of uncertainty. For the tests in the Virginia Tech supersonic wind tunnel, the first skin friction gage gave $\mathrm{C}_{\mathrm{f}}$ results with a standard deviation of $3.96 \%$ (see Table 2, section 4.4). The final skin friction gage, however, gave $C_{\mathrm{f}}$ results with a standard deviation of only $0.96 \%$, and a maximum scatter of $4.5 \%$ (see Table 4 , section 6.7). Individual measurements of $\mathrm{C}_{\mathrm{f}}$ can therefore be expected to fall within $\pm 2 \%$ of the average value $95 \%$ of the time for the final skin friction gage. Such statistical analysis of these results is somewhat less reliable due to the small sample size (less than 10 runs per gage). Some of the variation can be explained by differences in the flow conditions of the individual tests. Though the nominal tunnel conditions of the tunnel were kept the same, in practice 
it was very difficult to reproduce identical flow properties between tests. The fluctuations in the tunnel parameters would also be reflected in the standard deviation.

The $\mathrm{C}_{\mathrm{f}}$ results from the RBCC tests conducted with the first skin friction gage had a range of values that varied up to $12.4 \%$ from each other (see Table 3 , section 4.5.2). A disclaimer must be made about this, though. This skin friction gage was mounted in the combustor of the engine, and the fuel schedule was different for each run. Therefore no two runs were conducted with the same conditions at the gage location. Therefore, this number gives confidence to the results, but it cannot be used for error estimates.

The results from the HXEM tests of the final skin friction gage were a little more complicated. The standard deviation of the local $\mathrm{C}_{\mathrm{f}}$ results was $3.33 \%$, with a maximum scatter of $16.7 \%$ (see Table 5, section 6.8.2). The measured local $\mathrm{C}_{\mathrm{f}}$ for any given run could be expected to fall within $\pm 6.66 \%$ of the average $C_{f}$ for all runs $95 \%$ of the time. The tunnel conditions were intended to be identical from run to run, and the gage was mounted on the inlet, where the fuelling did not alter the flow. The repeatability of the gage itself however was not the only factor in these tests; there was the added variable of thermal drift in these tests. Accounting for the thermal drift, as described in section 6.8.2, was not perfect, and likely is to blame for some portion of the scatter.

\section{A.2 Thermal Drift}

The first two skin friction sensors of this study incorporated active cooling systems to help alleviate some uncertainty due to temperature sensitivity of the strain gages. The final skin friction gage, however, was completely uncooled. The result was that there was thermal drift in the output from this sensor during tests in the high enthalpy scramjet wind tunnel tests. A relatively simple method was employed to subtract out the thermal drift portion of the signal from those tests, and the results looked good. However, there was still an increased amount of scatter in those results (16.7\%) compared to results from the cold flow tests in the Virginia Tech supersonic wind tunnel (4.5\%). Certainly, the thermal drift and the subsequent post-processing must play a major part in contributing to this increased uncertainty.

It was attempted to calibrate the final skin friction sensor with temperature. Unfortunately, the temperature sensitivity of the final skin friction gage was non-linear and hard to interpret. Therefore, it is difficult to quote an error estimate per degree of 
temperature change. Rather, taking into account the uncertainty in the cold flow tests and the calibration, a conservative estimate of the uncertainty due to temperature effects would be 10-15\%, with the effect being the largest in hot, high enthalpy flows.

\section{A.3 Floating Element Errors}

\section{A.3.1 Misalignment Errors}

Allen's ${ }^{20}$ work showed that for a perfectly aligned floating element there was no error introduced into the measurement of skin friction. O'Donnell and Westkaemper ${ }^{33}$ similarly showed small errors for small misalignment; less than $2 \%$ for protrusions of 0.0005 inch $(0.00127 \mathrm{~cm})$. Hakkinen ${ }^{34}$ discussed a method for estimating the error caused by a protruding floating element. The equation, which was developed for a 2dimensional, rectangular floating element, is shown below:

$$
\varepsilon=(\Delta P / \tau)(h / l)
$$

Here $\Delta \mathrm{P}$ is the difference in pressure between the upstream and downstream sides of the floating element; $\tau$ is the wall shear stress; $h$ is the amount of protrusion; and 1 is the length of the floating element. Since the gages used in this study had cylindrical heads, the length 1 is replace with $\mathrm{d}$, the diameter of the floating head.

All of the checkout tests of the final skin friction gage took place on flat surfaces in supersonic flows. For these conditions there should have been no pressure gradient present. In this case, the error should be zero. Still, it is useful to provide a predictive estimate of the error that could be seen in a scramjet flow with a pressure gradient. Therefore, a pressure gradient that was observed by Chadwick, et $\mathrm{al}^{26}$ was used in the above equation. They quoted a pressure gradient of $1.5 \mathrm{psi} / \mathrm{ft}(34 \mathrm{kPa} / \mathrm{m})$ in a supersonic combustion wind tunnel experiment. Substituting this pressure gradient across the diameter of the floating element, and using the wall shear seen on the inlet of the HXEM tests, an error of less than $0.5 \%$ is predicted for a maximum protrusion of 0.0005 inches $(0.00127 \mathrm{~cm})$.

\section{A.3.2 Pressure Gradient Errors}

Pressure gradients can also induce errors even for a perfectly aligned floating element. In order to prevent this, the gaps of the first two skin friction gages were filled with silicon oil. Since the oil is an incompressible fluid, it makes it very difficult to 
establish a pressure difference from one side of the floating element to the other. The final skin friction gage, however, had no oil fill. It is more possible that a pressure gradient across the floating element could have induced errors in this configuration. As stated above, the checkout tests of the final skin friction sensor were conducted in zero pressure gradient environments. But, an estimate of the error that could be introduced due to a pressure gradient typical of a scramjet combustor was desired.

Hakkinen ${ }^{34}$ also discussed a crude method of estimating the error caused by a pressure gradient acting on a floating element. The equation is shown below:

$$
\varepsilon=\frac{(d P / d x) l \cdot t}{\tau \cdot l}
$$

Here 1 is the streamwise length of the floating element; $t$ is the effective gap depth; and $\tau$ is the shear stress. This equation was developed for a 2-dimensional floating element. Therefore, the quantity in the numerator is the force on the floating element per unit width due to pressure gradient. The quantity in the denominator is the shear force per unit width. To apply this equation to the final skin friction gage, the length, 1 , is taken to be equal to the diameter of the floating element ( 0.348 inches), and $t$ is taken to be equal to the height of the lip at the top of the floating element ( 0.030 inches). A pressure gradient considered representative of a scramjet combustor $(34 \mathrm{kPa} / \mathrm{m})$ was entered into the above equation along with a range of shear levels (150 to $800 \mathrm{~Pa})$ that might be experienced in a scramjet engine. The geometry of the final skin friction gage was used for 1 and $t$ (though 1 cancels out). This produced a range of estimated errors of 3 to 17\%, depending on the severity of the ratio of pressure gradient to shear level.

Two assumptions about the above equation make it a very conservative estimate. The first is that this equation assumes the floating element to have infinite width, isolating the upstream and downstream pressures. The 3-dimensional cylindrical shape of the actual floating element provides relief. The pressures upstream and downstream of the floating element are not isolated, but instead can equalize around the gap. The other assumption is that the pressure acting on the floating element remains constant across the entire height of the lip. Initial results of a numerical study of floating element errors indicate that the pressure varies around and across the cylindrical lip. These initial results 
also support the conclusion that a smaller lip height (C) greatly helps to reduce pressure forces on the floating element.

For the wind tunnel tests conducted with the final skin friction gage, the error from pressure gradients is believed to be very small (less than 3\%). An estimate of the errors for the scramjet flight test is desired, taking into consideration the above estimates and their limiting assumptions. It is believed that a worst-case estimate would indicate possible pressure gradient errors of up to roughly $15 \%$, with more likely moderate errors between 5 and $10 \%$.

\section{A.4 Uncertainty Summary}

Calculating the errors in direct measuring skin friction sensors is difficult. The uncertainty in the measurements reported here due to the calibration and misalignment effects has been shown to be small (1.6\% and $0.5 \%$, respectively). The results of the tests of the final skin friction gage in the supersonic wind tunnel were repeatable within $\pm 2 \%$, and were within $10 \%$ of previous tests and $10-15 \%$ of the integral method estimate. Differences in the test conditions and the estimate accuracy make these differences less worrisome. The pressure gradient effects for the tests conducted to date are believed to be less than $3 \%$. The largest source of uncertainty for the tests of the final skin friction gage is the thermal drift, which is believed to be 10 to $15 \%$. Adding the maximum of these together to get a worst-case estimate gives an error of about $25 \%$. More sensibly, calculating the root of the sum of the squares of these values gives an estimated error range of 11 to $16 \%$ for the high enthalpy scramjet wind tunnel tests. Doing the same with a larger pressure gradient that could be seen in the flight vehicle scramjet engine, the uncertainty range increases to 12 to $22 \%$. The errors encountered in flows without thermal effects and/or without pressure gradients, such as the supersonic cold flow tests, will be much less. 


\section{APPENDIX B:}

\section{FORTRAN CODE FOR HEAT FLUX GAGE POST-PROCESSING}

The heat flux gage incorporated two type $\mathrm{T}$ thermocouples wired in series. One of these was on the surface of the heat flux gage; the second was mounted inside the copper plug. A type $\mathrm{K}$ thermocouple was mounted adjacent to the second type $\mathrm{T}$ thermocouple. The temperature of the second type $\mathrm{T}$ thermocouple and the type $\mathrm{K}$ thermocouple were assumed to be the same. The voltage signal from the type $\mathrm{T}$ thermocouples corresponded to the temperature difference between the surface and backside thermocouple. In order to find the surface temperature from this signal, it was necessary to write a Fortran code to process the voltage. The code used to do this is attached below. The method was fairly simple. First the backside temperature was read from the type $\mathrm{K}$ thermocouple. Next it was necessary to determine the voltage from a type $\mathrm{T}$ thermocouple that would correspond to the backside temperature. The temperature and the voltage from a type $\mathrm{T}$ thermocouple are related through a $7^{\text {th }}$ order polynomial. The equation is written giving temperature as a function of voltage. To find voltage as a function of temperature, Newton's method was used to find the root of the $7^{\text {th }}$ order polynomial calibration equation given the backside temperature. The voltage signal measured from the two type $\mathrm{T}$ thermocouples wired in series was then added to the root. The resulting number, which was the output of the program, was the voltage for a type $\mathrm{T}$ thermocouple that corresponded to the surface temperature. Once calculated by the Fortran code, the surface temperature could be found by solving the $7^{\text {th }}$ order polynomial equation with this voltage. 


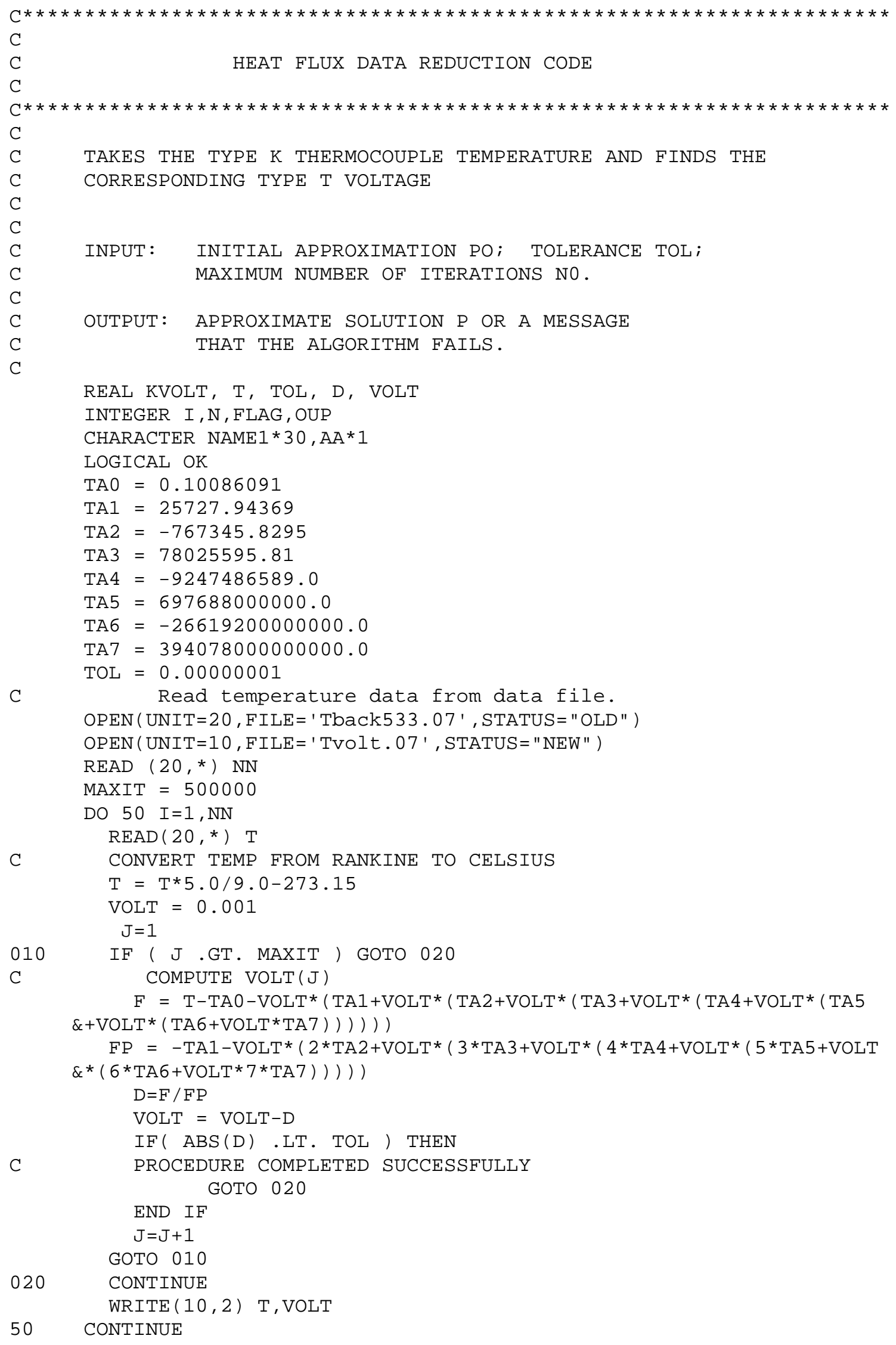


CLOSE (UNIT $=20)$

CLOSE (UNIT $=10$ )

STOP

2 FORMAT (1X, E15.8, 1X, E15.8)

3 FORMAT (1X,E15.8)

END 


\section{APPENDIX C:}

\section{NASA REPORTS}

NASA required several reports pertaining to the skin friction and heat flux gages installed on the Hyper-X flight vehicle. These are included below. The first two test reports originally contained appendices with test results. Since the test results have already been presented previously, those appendices have been omitted. 
C.1 Calibration Test Report 


\section{CALIBRATION TEST REPORT \\ FOR \\ HYPER-X SKIN FRICTION \\ AND \\ HEAT FLUX GAGES}

\section{PERFORMED/PREPARED BY \\ TED SMITH \\ VIRGINIA TECH \\ AEROSPACE \& OCEAN ENGINEERING \\ 215 RANDOLPH HALL \\ BLACKSBURG, VA 24061}

AUGUST 1, 2001 


\section{TABLE OF CONTENTS}

INTRODUCTION

TEST OBJECTIVE

TEST SEQUENCE

TEST RESULTS

APPENDIX A - TEST DATA 


\title{
INTRODUCTION
}

This test report documents the calibration effort for the Hyper-X Mach 10 skin friction and heat flux sensors developed by Virginia Tech, Aerospace \& Ocean Engineering Department. These sensors have been designed for testing in the engine of the third $\mathrm{X}$ 43A flight vehicle.

\section{TEST OBJECTIVE}

The objective of the calibration test program was to determine the sensitivities of the skin friction and heat flux gages to their design inputs, and any interfering inputs expected during flight-testing on the Hyper-X Research Vehicle.

\section{TEST SEQUENCE}

\author{
1.0 Static Weight Test (Skin Friction Gage Only) \\ 2.0 Angular Calibration (Skin Friction Gage Only) \\ 3.0 Pressure/Altitude Tests \\ 4.0 Thermal Tests \\ 5.0 Heat Loads Tests
}

\section{TEST RESULTS}

\subsection{Static Weight Test (Skin Friction Gage Only)}

A static calibration was performed on the skin friction sensor. The method used is illustrated in Figure 1. The method was a direct force application method. The gage was clamped in a horizontal position, and a cone was hung from the flow surface of the floating head with string and tape. Excitation of the strain gages was provided by the signal conditioning amplifiers, and the bridge output was balanced (trimmed to zero). Calibrated masses were then placed in the cone, and the corresponding output was read from a multimeter and recorded. Careful attention was given to ensure that the hanging weight was aligned with the desired axis of the skin friction sensor. This process was repeated to ensure repeatability of the sensor. Both positive and negative directions were calibrated along the sensing axis.

The input mass was converted to a force by simply multiplying by the gravitational constant $(\mathrm{F}=\mathrm{m} \cdot \mathrm{g})$. The force was then converted to a shear by dividing by the area of the flow surface of the floating head $\left(\tau_{\mathrm{w}}=\mathrm{F} / \mathrm{A}_{\text {head }}\right)$. This produced calibration curves that directly related the skin friction sensor output voltage to the shear acting on the floating head. Results of the calibration tests are shown in Appendix A.

This calibration procedure was repeated after each of the qualification tests to ensure that no change in the sensitivity had occurred. 


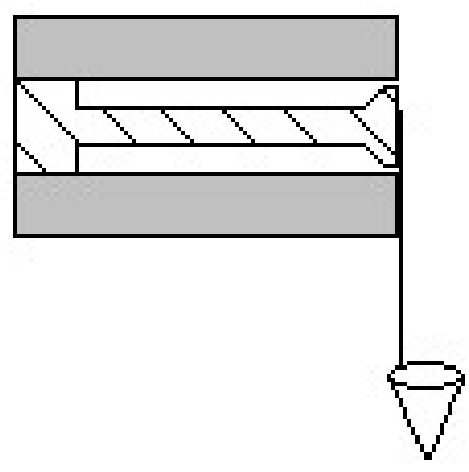

Figure 1: Static Calibration Method

The heat flux gage requires no calibration. It operates on first principles. It simply measures the temperature at the surface of the flow and at a point inside the copper wall a known distance from the surface. The time history of these two temperatures can be used to back out the heat flux into the wall.

\subsection{Angular Calibration (Skin Friction Gage Only)}

Since the skin friction gage was to be mounted on the Hyper-X Flight Vehicle (HXFV), and the vehicle would go be oriented at different angles during its flight, the gage was calibrated to determine any output due to angle changes. The gage was to be mounted in the vehicle such that the axis of the aircraft was aligned with the direction of motion of the floating element. This meant that changes in the pitch angle, or angle of attack, of the aircraft would cause the component of the gravity vector in the axial direction to change. This was the primary concern that warranted these tests, but the sensitivity to roll changes was also calibrated. Yaw angles cannot cause output from the sensor since they are about the same axis as the flexure ring hub.

The test data in Appendix A shows the orientation and notation of these angles. Results of these angular calibrations are also shown in Appendix A. The output due to changes in pitch angle (angle of attack) was small, about $40 \mathrm{mV}$ maximum. This was because of the counterweight. Theoretically, if the counterweight had been perfectly matched, there would have been no output at all. Though it was not possible to make a perfect counterweight, the output was satisfactorily small. The maximum output due to roll was about $55 \mathrm{mV}$. Also of note is that the output was greatest for large angular excursions from straight and level flight. If the output from the Wheatstone bridge is balanced before the flight when the vehicle is horizontal (zero pitch angle) and level (zero roll angle), then the gage output due to pitch and roll angles should be small. This calibration, however, makes it possible to subtract out the output due to these angles, should it be necessary.

\subsection{Pressure/Altitude Tests}

The altitude test was performed at NASA Dryden Flight Research Center. The skin friction and heat flux gages were placed in a pressure chamber. The wires for the gages 
were routed through a pass-through in the wall of the chamber to multimeters so that data could be recorded during the tests. A mass of 2 grams was hung from the floating head of the skin friction sensor to simulate a load. The chamber was then sealed and the pressure was reduced to correspond to different altitudes. Each altitude level was held for about 10 minutes before continuing to the next data point. It was planned to simulate up to $120,000 \mathrm{ft}$ as per HX-279, Rev. C, but the pressure chamber was not able to go any further than 96,000 $\mathrm{ft}$. The temperature in the chamber remained at ambient during the pressure tests.

Both gages passed this test with no problem. Neither gave any output due to changing pressures. This was expected since there are no sealed cavities. Visual inspection showed no damage or change in the physical appearance of the gages. The test data can be seen in Appendix A.

\subsection{Thermal Tests}

Thermal tests were performed on the skin friction and heat flux gages as per HX-279, Rev. C. The requirement states that the test articles are to be tested from $-40^{\circ} \mathrm{F}$ to $+160^{\circ} \mathrm{F}$ in operational mode. This was done at NASA Dryden and at Virginia Tech. The Virginia Tech test used a Blue M Electric ETC-04D-C Environmental Test Chamber with a Pro-550 Controller in Virginia Tech's Engineering Science and Mechanics Department.

\subsection{Transient Tests}

The NASA Dryden pressure chamber was used to do transient temperature tests. The skin friction and heat flux gages were placed in the chamber at ambient pressure, and the skin friction sensor output was balanced. Then a 2-gram weight was hung from the head of the skin friction gage in the same manner as during calibration. The temperature of the chamber was then reduced to $-40^{\circ} \mathrm{F}$. The temperature of the skin friction and heat flux gages was monitored using the thermocouples built into their housings. The output from the Wheatstone bridge of the skin friction sensor was also monitored. The temperature of the gages fell slower than the air in the chamber, due to the thermal mass of the sensors. The skin friction sensor fell more slowly than the heat flux gage since it is larger. As the temperature of the gages fell, the temperature and output of the gages were recorded.

The heat flux gage output did not change with temperature, as expected. The skin friction gage, however, did give output with temperature. At ambient temperature the output of the skin friction sensor was -0.4 volts, which corresponds to the 2 gram mass applied to the floating element. As the gage temperature fell, the output steadily increased in a nearly linear manner. The temperature of the skin friction gage was not allowed to reach a steady state, however. The output of the gage may have continued to change even after the temperature had reached a constant level. While the skin friction gage was still very cold, the chamber was opened, and the weight was removed from the floating element. The output of the gage increased from -0.08 volts to +0.32 volts, a change of +0.4 volts. This change in voltage due to the mass was the same as the calibration done at ambient conditions. This indicated that although the output had drifted due to temperature, the sensitivity had remained the same. 


\subsection{Steady-State Tests}

Steady state temperature tests were also attempted. These were done in Virginia Tech's Engineering Science and Mechanics Department using a Blue M Electric ETC-04D-C Environmental Test Chamber with a Pro-550 Controller. This test chamber has a twostage cascade refrigeration system and is capable of testing at temperatures from $-80^{\circ} \mathrm{C}$ to $+190^{\circ} \mathrm{C}$. The gages were placed in the chamber with wires routed through a passthrough. Output from the skin friction sensor and the thermocouples were read on multimeters.

For each test point the test chamber was programmed to reach and hold a steady temperature. The gages were then allowed to reach the temperature of the chamber and remain there for about 45 minutes before moving to the next test point.

Again the heat flux gage gave no output from temperature changes. And again the skin friction sensor did. The remainder of the discussion pertains only to the skin friction gage. The heat flux sensor was considered to have passed the temperature test with no problem, while further investigation was needed for the skin friction sensor.

When the temperature was changed the output from the skin friction gage would go through a transient response before approaching a steady value. Both the maximum transient value and the steady value were recorded for each test point. The transient output was always greater than the steady value. Like the tests in the Dryden test chamber, the output increased with decreasing temperature, and decreased with increasing temperature. The output did not continue to increase at the coldest temperature. Similarly, the output did not continue to decrease at the hottest temperature. After the hottest temperature test point, the chamber was returned to ambient temperature. The gage output, however, did not return to zero during the following few hours.

In order to study this more thoroughly, the hottest data point $\left(160^{\circ} \mathrm{F}\right)$ was repeated over a much longer time period. The chamber and gage were allowed to remain at the test temperature for well over an hour. The chamber was then opened to the atmosphere. The gage was allowed to slowly reach ambient temperature overnight. The temperature and output from the gage were recorded during the following 22 hours.

See the test data chart for the $2^{\text {nd }}$ temperature test in Appendix A. The data points were recorded chronologically in the clockwise direction, starting from the ambient temperature. The data points on the upper portion of the chart that are connected by the line were recorded before the test chamber was opened to the atmosphere. The data points after the break in the line were recorded as the gage cooled after the test chamber was opened. The upper portion of the graph shows the initial rise in the output during the transient response, and then the approach to a steady level of about -0.1 volts. Once the chamber was opened, the voltage fell a small amount as the gage began to cool. It was at this point that the gage was left overnight. When the measurements were resumed in the 
morning, the temperature had returned to ambient, and the output also had approached zero.

Once again, the gage was calibrated after the temperature tests. These calibrations showed that the exposure to the hot and cold temperatures did not alter the calibration of the sensor. Visual inspection showed no damage or change in the gage.

These temperature tests were of limited use. The actual flight temperature environment is transient and very difficult to reproduce in a lab. And the steady-state response of the gage does not help much in predicting the transient response. Also, since the gage is to be mounted in the engine of the flight vehicle, the temperatures that it sees may be well above the $160^{\circ} \mathrm{F}$ that was specified for these environmental tests. Therefore the behavior of the skin friction gage in the flight may or may not be like the behavior in these tests. The most important fact that was determined from these tests was that the output of the gage did change with temperature, but the sensitivity did not.

The test data can be seen in Appendix A.

The two gages are considered to have passed the temperature tests. For the flight test the skin friction gage will require post-processing to account for thermal drift in its signal.

\subsection{Heat Loads Tests}

It was desired to test the skin friction and heat flux gages in a heating environment closer to that expected in the scramjet engine during flight. To do this it was necessary to reproduce the wall heat flux that was estimated for the engine near the location of the sensors. Two facilities were used to attain this goal. Both facilities used radiative heat flux sources. The heat to the engine wall during the flight test, however, will be transferred via convection. Methods for accurately reproducing high levels of convective heat fluxes are not available. The best way to accurately simulate high heating loads, then, is to do so with calibrated radiative systems.

\subsection{NASA Dryden Tests}

The first set of heat load tests was performed at NASA Dryden Flight Research Center in the Flight Loads Laboratory. This laboratory is equipped with a heat flux gage calibration system that can reproduce high heat flux levels. The equipment includes a Thermogage Model $48 \mathrm{KW}$ Furnace and a flat plate heater assembly. The furnace provides a high power voltage across a thin, flat graphite plate. The current flowing through the flat plate causes it to heat to extreme temperatures. At these extreme temperatures, the plate glows and radiates heat flux values up to $400 \mathrm{Btu} / \mathrm{ft}^{2} \bullet \mathrm{s}$. A calibrated Gardon-type heat flux gage is placed on one side of the flat plate to monitor the heat flux level. The test article is placed on the opposite side and at the same distance from the flat plate in order to be exposed to the same heat flux.

For these tests a special mounting bracket was constructed for the skin friction gage. This consisted of a one-inch thick copper block to simulate the surrounding engine wall. 
The holes for the heat flux gage (or the skin friction gage floating element) and $1 / 4-20$ mounting screws were machined into this block so that the heat flux gage (or floating element) would be flush with the surface. The flat plate heater was oriented vertically, so the copper block was designed to be vertically mounted such that the surface would be parallel to the flat plate. An aluminum plate was fastened to the copper block so that the entire fixture could be clamped in place. Figure 2 presents a sketch of the skin friction gage mounted in the copper block mounting assembly opposite the flat plate heater. Only the electrodes and the graphite flat plate itself are shown in the sketch for simplicity. When mounted in this configuration the surface of the floating element and the copper plate are exposed to the heat flux in the same manner as would be in the Hyper-X engine.

To simulate the heating environment of the flight vehicle it was desired to expose the gage to a step increase in heat flux, hold that level for several seconds, and then return to zero heat flux. This would reproduce the opening of the engine cowl at Mach 10 and the firing of the engine for several seconds before the cowl is closed again. There is no flow through the engine, and therefore no heat flux, before and after the cowl is opened and closed.

However, this proved difficult to reproduce in the Dryden Flight Loads Laboratory. The flat plate heater ramps up to the test heat flux level over about 25 seconds. This length of time is about the same as the total engine-on time of the flight test. A shutter system was designed to raise and lower a graphite plate from between the heater plate and the copper block. It was hoped that this could be used to shield the gage during the ramp-up and

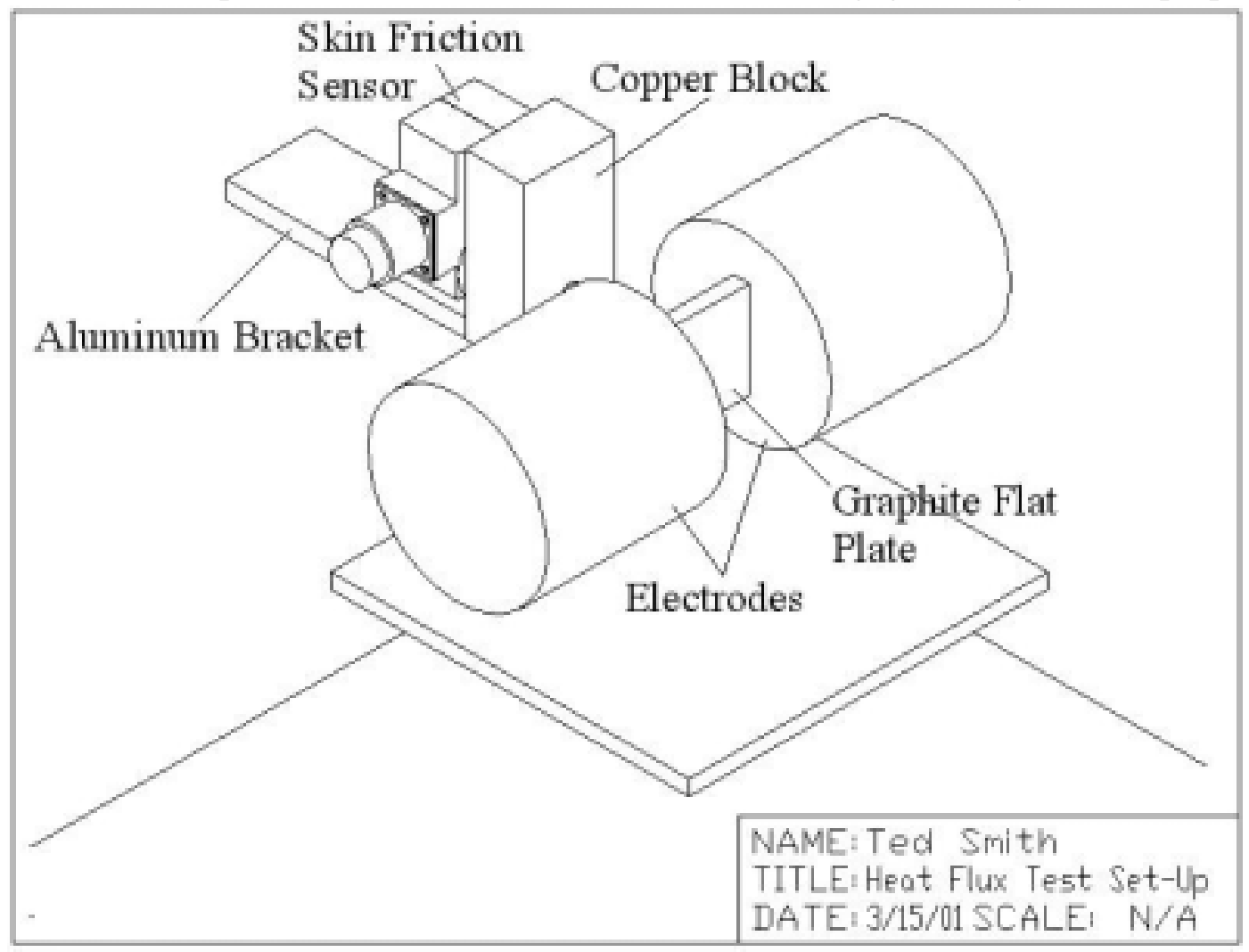

Figure 2: Heat Load Test Setup Sketch 
ramp-down periods. Unfortunately, the high temperatures proved to be too much for the shutter system, and the idea had to be abandoned.

A second difficulty also prevented the Dryden tests from providing useful information. The flat plate heater was easily capable of radiating the heat flux levels that were predicted for the flight test. These levels were between 70 and $130 \mathrm{Btu} / \mathrm{ft}^{2} \mathrm{~s}$. But the surface of the copper block, the heat flux gage, and the floating element are machined copper, which has an emissivity of between 0.07 and 0.15 . This meant that the copper block, heat flux gage, and floating head were absorbing only roughly $10 \%$ of the radiated heat flux.

Because of the emissivity problem, there was considerable uncertainty in the amount of heat flux that was absorbed by the sensor. It also meant that the absorbed heat flux was much less than the predicted level for the flight test. And the lack of a shuttering system led to exposure of the sensor to the heat flux for periods much longer than the expected test time for the flight. These problems with the tests at NASA Dryden led to a second set of tests.

\subsection{Vatell Tests}

The second set of tests was performed at Vatell Corporation in Christiansburg, Virginia. Vatell manufactures heat flux sensors and also performs heat flux tests and calibrations. A flat plate heater facility very much like the one at NASA Dryden was used at Vatell to reproduce the predicted heat flux of the Hyper-X flight test.

An added capability at Vatell was the ability to traverse the test article on rails toward and away from the flat plate heater during testing. This allowed the skin friction and heat flux gage to be pulled away from the heater plate and shielded during the ramp-up and shutdown periods. In this way the gages could be exposed to the heat flux only for the brief amount of time expected during the flight test.

It was also required that the gages should absorb the entire amount of radiated heat flux. To ensure this, the surface of the gage and the copper block were coated with soot. The emissivity of the surface of the copper block and floating element when coated with the black soot was very near 1.0. This meant that during the heat load tests at Vatell the sensors absorbed almost the entire amount of heat flux radiated by the flat plate. Figure 3 shows the heat flux gage mounted in the copper block and coated with soot prior to testing.

The skin friction sensor was tested at 30 and $70 \mathrm{Btu} / \mathrm{ft}^{2} \mathrm{~s}$. Before each run the output from the skin friction sensor was balanced to zero. The output from the Wheatstone bridge and the type $\mathrm{K}$ thermocouple in the housing were recorded on a PC via a National Instruments $\mathrm{A} / \mathrm{D}$ board using LabView data acquisition software. The heat flux gage was tested separately at 30,70 , and $100 \mathrm{Btu} / \mathrm{ft}^{2} \mathrm{~s}$. The voltage from the surface and backside type $\mathrm{T}$ thermocouples wired in series and the backside type $\mathrm{K}$ thermocouple were recorded on the same PC. 


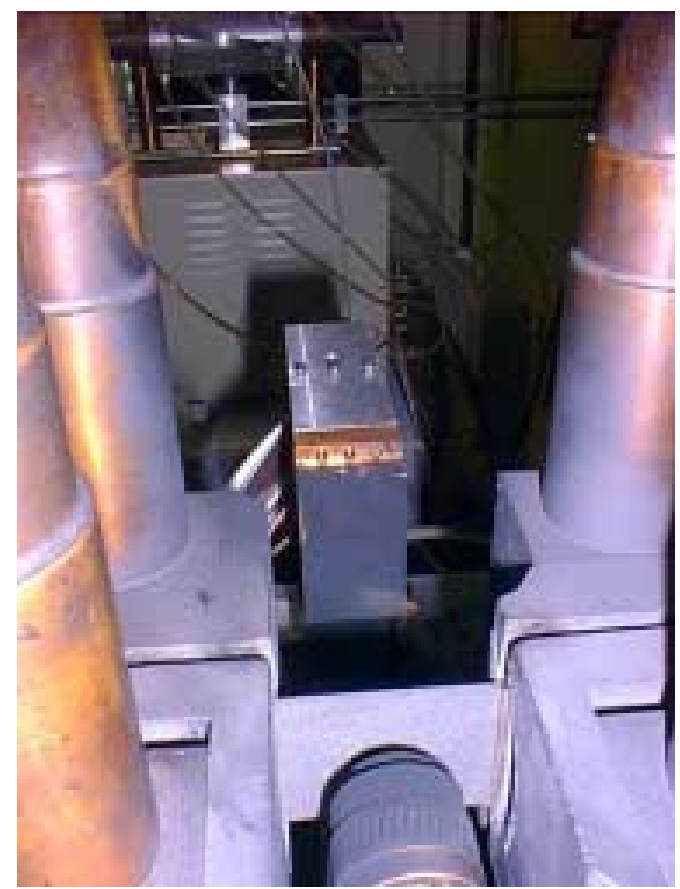

Figure 3: Heat Flux Gage in Test Fixture Coated with Soot

While the heater plate was ramped up to the test point the skin friction or heat flux gage and copper mounting block were covered with a tin sheet and traversed on rails away from plate. Once the flat plate reached the designated heat flux, the shielding was removed and the gage was traversed up to the flat plate for several seconds. Then the gage was traversed away from the plate again and the power to the heater plate was cut. Figure 4 shows the skin friction gage withdrawn from the heater plate and traversed up to the flat plate. Figure 5 shows the gage withdrawn from the plate and shielded as the flat plate heats up.

\subsubsection{Skin Friction Gage Tests}

Results of these tests are shown in Appendix A. The first tests were of the skin friction gage, first at 30 and then at $70 \mathrm{Btu} / \mathrm{ft}^{2} \mathrm{~s}$. The figures show the temperature and Wheatstone bridge output from the skin friction gage over time. The temperature was measured by the thermocouple mounted in the housing. The large spikes in the bridge output are due to the test fixture hitting the stops of the guide rails as the fixture was traversed towards and away from the flat plate. These impulsive loads effectively mark the beginning and end of the heat flux exposure period. Several seconds of data were recorded before and after the exposure period.

Before the first test exposure the output from the sensor was steady near zero and the temperature was steady at room temperature as seen in the chart. At about eight seconds into the run, the shielding was removed and the gage was moved within range of the flat plate heater. The gage was in the test position until about 15 seconds into the run, for a total exposure time of about seven seconds. Then the heater was powered down and the gage was withdrawn from the plate. The temperature and bridge output remained nearly unchanged during the first several seconds of the test time. Then, after several seconds, 


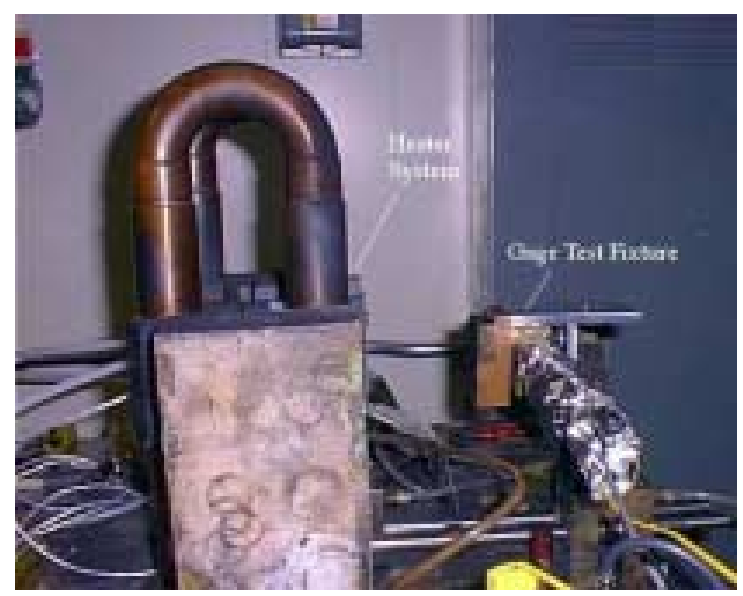

a)

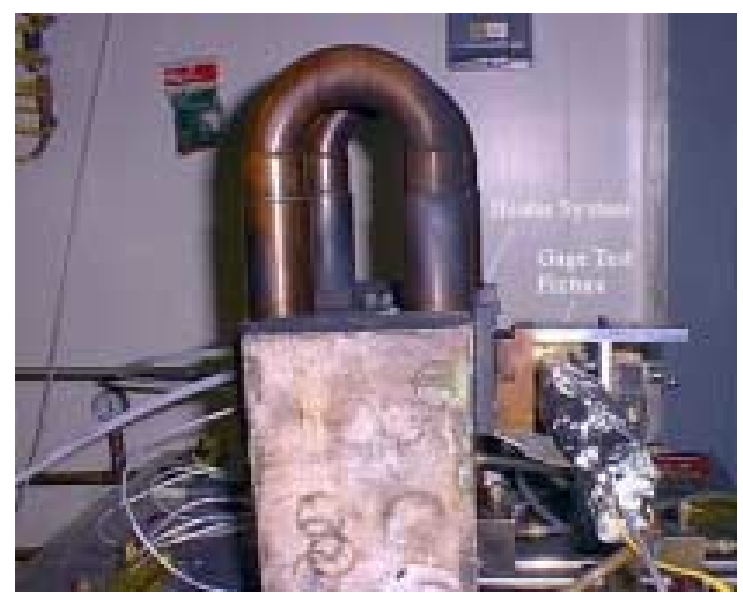

b)

Figure 4: Test Fixture a) Withdrawn and b) Up Close to Heater Plate

the temperature and the output from the skin friction sensor began to rise, the temperature rising from about $80^{\circ} \mathrm{F}\left(27^{\circ} \mathrm{C}\right)$ to about $90^{\circ} \mathrm{F}\left(32^{\circ} \mathrm{C}\right)$ and the gage output approaching a maximum offset of about $45 \mathrm{mV}$. The major portion of the temperature and output rise occurred after the exposure time and both seemed to follow the same basic trend.

For the second skin friction gage test the heat flux was $70 \mathrm{Btu} / \mathrm{ft}^{2} \mathrm{~s}$. Again the temperature measured in the housing of the gage and the output from the strain gages are presented in Appendix A. The heat flux exposure period for this test can be seen from the spikes in the gage output, once again caused by the test fixture hitting the stops of the rail traverse system. The beginning of the exposure period was at about 15 seconds into the run, and the end of the exposure period was at about 22 seconds, for a total test time of about 7 seconds. The strain gage output began at about $-12 \mathrm{mV}$ and began to rise after about 5 seconds of exposure to the high heat flux. Likewise the temperature began at about $90^{\circ} \mathrm{F}\left(32^{\circ} \mathrm{C}\right)$ and began to increase after several seconds of exposure. Similar to the first test, the major portion of the drift in temperature and strain gage output occurred after the test window. The temperature continued to increase up to about $115^{\circ} \mathrm{F}\left(46^{\circ} \mathrm{C}\right)$ and the Wheatstone bridge continued to drift up to about $110 \mathrm{mV}$. These shifts occurred 


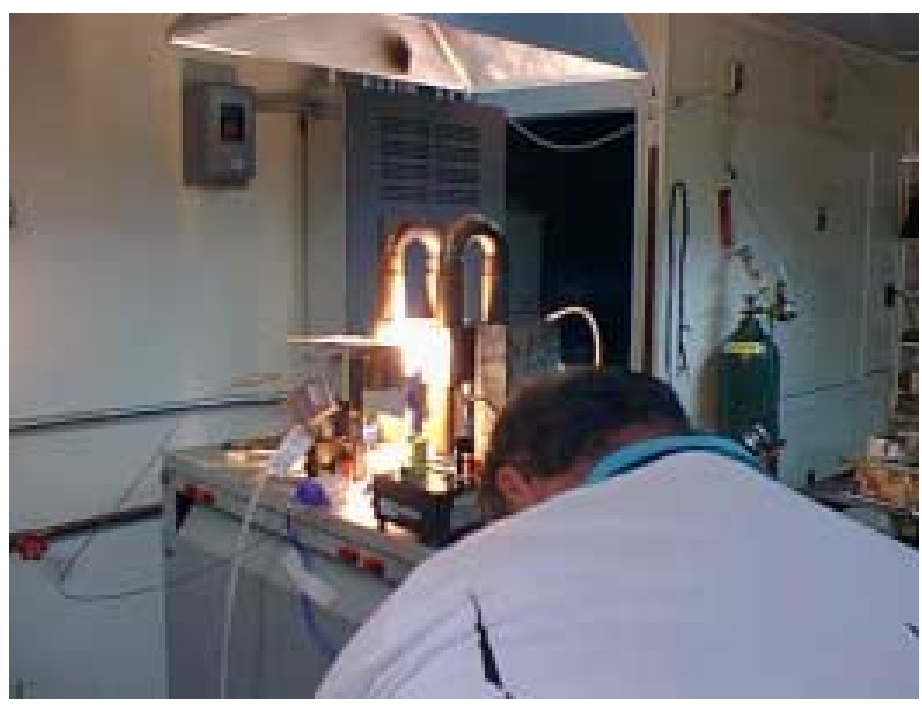

Figure 5: Heater Plate Coming Online, Test Fixture Shielded

more rapidly and to greater extremes than the during the lower heat flux test, as expected. The internal temperature and the gage output were monitored for several minutes after the test. The temperature reached a maximum of about $140^{\circ} \mathrm{F}\left(60^{\circ} \mathrm{C}\right)$ after a few minutes, before slowly cooling back down. The gage output reached about $150 \mathrm{mV}$ and then slowly returned towards zero.

These tests showed that the skin friction sensor did not give an output directly related to heat flux. However, the output did drift due to the temperature increase inside the gage. The increase in temperature and the subsequent drift in the strain gage signal were delayed until several seconds after the initial exposure to the heat flux. Tests of a heat flux gage in the same test fixture (to be discussed later) showed that the temperature of the surface of the copper block reached about $270^{\circ} \mathrm{F}\left(132^{\circ} \mathrm{C}\right)$ during a $70 \mathrm{Btu} / \mathrm{ft}^{2} \mathrm{~s}$ test, and it is assumed that the temperature of the surface of the floating element reached a similar temperature. This means that although the surface of the floating element may get very hot, the sensor a large enough thermal mass that the thermal wave takes a relatively long time to propagate through the sensor. This was considered to be very good because of the relatively short duration of the engine firing during the flight test. The cowl of the engine will only be open for about 15 seconds during the entire flight, and the engine will only be fired for about 7 of those seconds. Any thermal drift from the skin friction gage during that time period should be minimal and/or easily accounted for in post-processing. This very situation was encountered later during wind tunnel tests.

After the heat load tests the skin friction gage was recalibrated to determine if the exposure to the heat flux had in any way altered its sensitivity. These calibrations once again showed that the sensitivity remained unchanged. Visual inspection of the sensor showed no damage to the floating element, the gaskets, or any other component of the gage. The gage was not sensitive to the heat flux and was able to survive that hot environment. 


\subsubsection{Heat Flux Gage Tests}

The heat flux gage was tested at 30,70 , and $100 \mathrm{Btu} / \mathrm{ft}^{2} \mathrm{~s}$. The surface type $\mathrm{T}$ thermocouple and the backside type $\mathrm{T}$ thermocouple were wired in series to produce a millivolt signal corresponding to the temperature difference between the surface and the backside. A type $\mathrm{K}$ thermocouple adjacent to the backside type $\mathrm{T}$ thermocouple was acquired for a reference temperature. There is a polynomial equation that relates temperature to the millivolt signal that a type T thermocouple would produce. Given the backside temperature from the type $\mathrm{K}$ thermocouple, a root-finding Fortran code was run to solve for the corresponding type $\mathrm{T}$ thermocouple voltage. The voltage from the type $\mathrm{T}$ thermocouple in series was then added to this voltage to find the voltage that corresponded to the surface temperature. The polynomial was then solved to determine this temperature.

See Appendix A. For each test, the backside and surface temperatures are the same before the exposure period. Then the surface temperature jumps as the gage is exposed to the heat flux. The backside temperature climbs more slowly. When the heater plate is shut down, the surface temperature drops quickly, while the backside temperature drops more slowly. The surface temperature approaches the backside temperature as the temperature in the sensor becomes uniform. For the first test, the surface temperature reached a maximum of about $180^{\circ} \mathrm{F}$, while the temperature difference approached about $60^{\circ} \mathrm{F}$. For the second test, the surface temperature reached a maximum of about $280^{\circ} \mathrm{F}$, while the temperature difference approached $140^{\circ} \mathrm{F}$. In the last test, the surface approached $400^{\circ} \mathrm{F}$, and the temperature difference was just over $200^{\circ} \mathrm{F}$.

The heat flux gage gave good output during these tests. The temperature histories make sense. Further analysis of the data is to be done by Greg Walker at Vanderbilt University. He will run a code to back out the heat flux levels from the temperature histories. Inspection of the gage afterwards showed no damage to the gage.

\section{POST TEST STATUS}

The skin friction and heat flux gages have completed all of the above mentioned calibration tests. They have also been tested in the Virginia Tech 9-inch by 9-inch supersonic wind tunnel. Finally, these sensors were successfully tested in the Hyper-X Engine Model (HXEM) at NASA Langley in the Arc-Heated Scramjet Test Facility (AHSTF). Results from all of these tests were very good. The gages survived all of the tests with no damage or change in calibration. The measurements obtained from the HXEM tests were in agreement with expectations and predictions.

One skin friction gage and one heat flux gage are currently ready for installation in the engine. A second skin friction gage was fitted with a special adapter for the HXEM tests. This sensor needs to be reassembled for the flight vehicle configuration. This should occur by no later than $8 / 17 / 01$.

All that remains is for final approval of the qualification of the sensors and the installation in the flight vehicle. 
C.2 Qualification Test Report 


\title{
QUALIFICATION TEST REPORT \\ FOR \\ HYPER-X SKIN FRICTION \\ AND \\ HEAT FLUX GAGES
}

\author{
PERFORMED/PREPARED BY \\ TED SMITH \\ VIRGINIA TECH \\ AEROSPACE \& OCEAN ENGINEERING \\ 215 RANDOLPH HALL \\ BLACKSBURG, VA 24061
}

JULY 31, 2001 


\section{TABLE OF CONTENTS}

INTRODUCTION

TEST OBJECTIVE

TEST SEQUENCE

TEST RESULTS

POST TEST STATUS

APPENDIX A - TEST DATA 


\section{INTRODUCTION}

This test report documents the qualification effort for the Hyper-X Mach 10 skin friction and heat flux sensors developed by Virginia Tech, Aerospace \& Ocean Engineering Department. These sensors have been designed for testing in the engine of the third X43A flight vehicle. The sensors were tested to the specifications of HX-279, Revision C where possible. When the specifications of HX-279, Rev. C were not possible, the reasons are presented, as well as any actions undertaken to perform a similar test.

\section{TEST OBJECTIVE}

The objective of the qualification test program was to verify that the skin friction and heat flux gages would meet the requirements necessary for approval for flight-testing on the Hyper-X Research Vehicle.

\section{TEST SEQUENCE}

6.0 Pressure/Altitude Tests

7.0 Thermal Tests

8.0 Vibration Tests

\section{TEST RESULTS}

\subsection{Pressure/Altitude Tests}

The altitude test was performed at NASA Dryden Flight Research Center. The skin friction and heat flux gages were placed in a pressure chamber. The wires for the gages were routed through a pass-through in the wall of the chamber to multimeters so that data could be recorded during the tests. A mass of 2 grams was hung from the floating head of the skin friction sensor to simulate a load. The chamber was then sealed and the pressure was reduced to correspond to different altitudes. Each altitude level was held for about 10 minutes before continuing to the next data point. It was planned to simulate up to $120,000 \mathrm{ft}$ as per HX-279, Rev. C, but the pressure chamber was not able to go any further than $96,000 \mathrm{ft}$. The temperature in the chamber remained at ambient during the pressure tests.

Both gages passed this test with no problem. Neither gave any output due to changing pressures. This was expected since there are no sealed cavities. Visual inspection showed no damage or change in the physical appearance of the gages. The test data can be seen in Appendix A.

\subsection{Thermal Tests}

Thermal tests were performed on the skin friction and heat flux gages as per HX-279, Rev. C. The requirement states that the test articles are to be tested from $-40^{\circ} \mathrm{F}$ to $+160^{\circ} \mathrm{F}$ in operational mode. This was done using a Blue M Electric ETC-04D-C Environmental 
Test Chamber with a Pro-550 Controller in Virginia Tech's Engineering Science and Mechanics Department.

The heat flux gage gave no output due to temperature changes. Visual inspection showed no sign of damage or change in the appearance of the gage.

The skin friction gage also showed no sign of damage or change in appearance. The skin friction gage did give output with temperature, but its calibration did not change. The temperature sensitivity of the skin friction gage was investigated thoroughly. The test data can be seen in Appendix A, and the reader is also referred to the Calibration Test Report for the skin friction and heat flux gages for a thorough discussion.

The two gages are considered to have passed the temperature tests. For the flight test the skin friction gage will require post-processing to account for thermal drift in its signal.

\subsection{Vibration Tests}

The heat flux gage was not shaken. Its current configuration includes long thermocouple shafts that extend from the base of the gage. If shaken in this configuration, the thermocouple shafts could be damaged. However, the thermocouple shafts will be bent when installed into the Hyper-X engine. They will also be rigidly mounted so that they cannot deflect. In the installed configuration, damage to the thermocouple shafts due to vibration is unlikely. Since there were only a couple of these sensors, it was not possible to bend the thermocouples just for the shaking test, since they would then not be able to be installed in the engine. The rest of the heat flux sensor is rigid and unlikely to be susceptible to vibrational damage. Therefore the vibrational test effort was concentrated on the skin friction gage, which has moving parts that are more of concern in a vibrational environment.

The skin friction sensors were shaken in the Virginia Tech Modal Analysis Lab. The skin friction gage was fastened to a shaker (Vibration Test Systems VG 100-41), and an accelerometer (Kistler 8630B50) was mounted onto the skin friction gage. As usual, the 2310 signal-conditioning amplifier powered the skin friction gage. An HP 35665A Digital Signal Analyzer (DSA) was used to supply the source signal for the shaker and to acquire and process the signals from the accelerometer and the skin friction gage. An Onkyo Integra M-504 stereo power amplifier amplified the source signal from the DSA.

The first test was the test of survivability. Since the flexure ring was the only moving part in the sensor, it was the part that had the greatest possibility of failure. Therefore a skin friction sensor was assembled with a flexure ring that had no strain gages mounted onto it. In case the flexure ring did fail, it would not be one that had already been instrumented with strain gages.

It was intended to shake according to HX-279, Rev. C, which calls for a random vibration at $8 \mathrm{~g}_{\mathrm{rms}}$ from 15 to $2000 \mathrm{~Hz}$. The equipment was able to produce a random noise signal, but the vibration was not uniformly $8 \mathrm{~g}_{\mathrm{rms}}$ at all frequencies, but only at some 
frequencies. Therefore the dummy skin friction gage was shaken with a sine wave at discrete frequencies of 15, 100,150,300,500,1000,1500, and $2000 \mathrm{~Hz}$ at levels of 2 and $8 \mathrm{~g}_{\mathrm{rms}}$. In this way it was possible to ensure that the level of shaking was $8 \mathrm{~g}_{\mathrm{rms}}$ for each frequency tested. This was done along all three geometric axes. The total time shaken along each axis at $8 \mathrm{~g}_{\mathrm{rms}}$ was roughly 6.5 minutes.

Before the above tests were done, a failure mode test was done. It was discovered that at extremely high g-loads (20-50 $\mathrm{g}_{\mathrm{rms}}$ ) a vibrational mode could be excited that could lead to the failure of the ring, but only if the floating head was not constrained. In this manner a non-instrumented flexure ring was shaken to failure. The webs of the flexure ring were shorn. Even after the webs were broken, however, the hub remained clamped in the housing, and the ring itself remained intact. Therefore the ring and the components attached to it (the adapter, floating head, and counterweight) were constrained within the housing and were not able to come out. This was important because it showed that even if the flexure ring were to fail during the flight test, that the sensor would not come apart and pose a risk to any other part of the vehicle. See Appendix A for images of the broken ring being constrained within "Housing b" after the gage was disassembled for inspection. Even more importantly, this failure mode can be avoided. When a sleeve with the same diameter as that of the engine wall mounting hole was fitted over the floating element, the floating head would strike the sleeve at high loads, but would not fail since the deflections were constrained. Once the floating head was constrained in this manner, the gage survived the tests at the aforementioned frequencies and g-loadings without any problem. Visual inspection of the flexure ring after these tests did not reveal any distortion of the webs. For the actual flight test, the mounting hole in the engine will act as a mechanical stop in the same manner, and prevent failure of the flexure ring due to vibrational loads.

Since the survivability tests were done with non-instrumented flexure rings, no data was recorded from the skin friction gage during those tests. Once the dummy gage survived the shaking tests at high levels, a gage with an instrumented flexure ring was shaken. The purpose was to determine the frequency response of the skin friction sensor. These tests were done at lower g-loadings of about $1 \mathrm{~g}_{\mathrm{rms}}$. This allowed the frequency response of an instrumented flexure ring to be determined without exposing it to the higher load levels.

For these tests, the skin friction sensor was mounted to the shaker and the DSA recorded the output from the skin friction sensor. The source signal that was sent to the shaker was a burst-chirp sine wave sweep from 15 to $3215 \mathrm{~Hz}$. The DSA produced frequency response function charts during these tests, where the input $(\mathrm{F})$ was taken to be the source signal that was sent to the shaker, and the output (X) was taken to be the signal from the skin friction gage. This was done for all three geometric axes of the sensor.

After the vibration tests were done the skin friction gage was calibrated with the static calibration method. It was important to know whether or not the shaking had in any way altered the calibration or sensitivity of the sensor. It was found that the calibration of the 
sensor after the shaking tests was identical to the calibration done beforehand. The shaking had not affected the sensitivity of the gage.

See Appendix A for the test data.

\section{POST TEST STATUS}

The skin friction and heat flux gages have completed all of the above mentioned qualification tests. They have also been calibrated, tested in radiative heat loads facilities, and tested in the Virginia Tech 9-inch by 9-inch supersonic wind tunnel. Finally, these sensors were successfully tested in the Hyper-X Engine Model (HXEM) at NASA Langley in the Arc-Heated Scramjet Test Facility (AHSTF). Results from all of these tests were very good. The gages survived all of the tests with no damage or change in calibration. The measurements obtained from the HXEM tests were in agreement with expectations and predictions.

One skin friction gage and one heat flux gage are currently ready for installation in the engine. A second skin friction gage was fitted with a special adapter for the HXEM tests. This sensor needs to be reassembled for the flight vehicle configuration. This should occur by no later than $8 / 17 / 01$.

All that remains is for final approval of the qualification of the sensors and the installation in the flight vehicle. 
C.3 Handling and Cleanliness Report 


\title{
HANDLING AND CLEANLINESS REQUIREMENTS FOR HYPER-X SKIN FRICTION AND \\ HEAT FLUX GAGES
}

\author{
PERFORMED/PREPARED BY \\ TED SMITH \\ VIRGINIA TECH \\ AEROSPACE \& OCEAN ENGINEERING \\ 215 RANDOLPH HALL \\ BLACKSBURG, VA 24061
}

AUGUST 1, 2001 


\section{TABLE OF CONTENTS}

INTRODUCTION

1.0 SKIN FRICTION GAGE

1.1 HANDLING

1.2 INSTALLATION

1.3 FUNCTIONAL TESTING

1.4 CLEANLINESS

2.0 HEAT FLUX GAGE

2.1 HANDLING

2.2 INSTALLATION

2.3 FUNCTIONAL TESTING

2.4 CLEANLINESS 


\section{INTRODUCTION}

This document provides instructions for the safe handling of the Hyper-X Mach 10 skin friction and heat flux sensors developed by Virginia Tech, Aerospace \& Ocean Engineering Department. These sensors have been designed for testing in the engine of the third X-43A flight vehicle.

\subsection{SKIN FRICTION GAGE}

The Virginia Tech skin friction sensor design consists of several components. The most important of the internal components is the flexure ring. This is the only moving part in the gage, and provides the mechanism by which the shear force is measured. It does this by deflecting under a load, and strain gages mounted on the flexures produce an output proportional to the load. The load is transferred to the flexure ring by means of a copper floating head that extends out of the brass housing, through the engine wall, and is flush with the flow surface of the engine. Shear forces acting on the wall also act on the surface of the floating head, causing it to deflect.

\subsection{HANDLING}

This sensor should be handled with the same care that would be given to any other expensive and unique piece of instrumentation. It should be stored and packaged in padded containers when not in use. Special handling considerations are described below.

The flexure ring is designed to deflect under the load expected in the flow. It is a sensitive piece of equipment. It is therefore, the most important part of the sensor to protect. Since the floating head protrudes out of the housing and is rigidly connected to the flexure ring, it presents the easiest way by which to transmit accidental damaging forces to the flexure ring.

In order to prevent this, the skin friction sensor is to be stored and transported with a sleeve that fits over the floating head mounted to the housing. This sleeve will be provided with the sensor. It has the same diameter as the hole in the engine in which the floating head is to be mounted. This prevents the floating head from being able to deflect far enough to damage the flexure ring. The only time that the sleeve is to be removed is during installation into test fixtures.

\subsection{INSTALLATION}

During installation of the gage into the engine or other test fixture, care should be taken not to place excessive loads on the floating element. As the sensor is mounted into the engine, the floating element is inserted into the hole in the engine wall. During this process the floating element should be carefully aligned such that it enters the hole straight. Hitting the sides of the hole with the floating head may not be totally avoidable, but it should be minimized as much as possible.

Once the gage is installed in the engine, the mounting screws can be fastened. The floating head should be monitored as the screws are tightened to make sure that the head 
remains centered in the hole. If gage were to be tightened down such that the floating head were hitting the side of the hole, the sensor would not be able to measure the shear during the flow. Deflecting the head by lightly running a finger over it can be done to verify that the head is not hitting the engine wall. It should be possible to see the head deflect about one thousandth of an inch in the positive and negative directions.

Once the gage is installed and the screws have been tightened with the floating head centered in the hole, a final alignment should be done. There are 1/16" dowel pin through holes in the gage housing adjacent to the $1 / 4-20$ mounting screw clearance holes. A drill can be run through these dowel pin holes and a small depth into the engine wall. Then dowel pins can be press fit through the housing into the engine for final alignement.

\subsection{FUNCTIONAL TESTING}

Functional testing of the skin friction sensor can still be performed with the sleeve mounted on the sensor, or with the sensor mounted in the engine. With the gage powered on, an output can be produced simply by gently running a finger over the floating head surface in the direction of the flow. Motion in the forward and backward directions should produce positive and negative output, respectively (or vice versa, depending on mounting configuration).

\subsection{CLEANLINESS}

Once the skin friction gage is mounted in the engine, care should be taken to prevent debris from entering the gap between the engine wall and the floating element. Such debris could deprive the floating head from its full range of motion.

To prevent such debris, it is suggested that tape be placed over the floating head and the gap surrounding it in the engine wall. It is also undesirable for adhesive to get around the surface of the floating head and the gap. Therefore a large piece of tape should be used such that a large area around the gage location is covered, and a non-adhesive shield (a piece of paper would work) should be placed between the tape and the floating head and gap. In this way the tape will adhere to the area surrounding the floating head without adhering to the floating head itself. The tape cover may need to be periodically replaced if it becomes dirty or loses adhesion. Remember to remove the tape before the flight test!

In the case that some debris should enter into the gap around the floating head, it may be possible to remove it. If it is large enough, it may be possible to carefully remove it by hand, with tweezers, or with an Exacto knife, taking care not to put excessive loads on the floating head. If this is not possible, blowing air into the gap may force the debris out. Functional testing can be used to determine if debris is restricting the sensor from its full range of output.

\subsection{HEAT FLUX GAGE}

The heat flux gage is much smaller and simpler than the skin friction gage. It has no moving parts. It basically consists of a copper slug fitted with three thermocouples; one that mounts flush with the flow surface, and two that measure inside the slug. By 
measuring the temperature difference between the engine surface and a point at a known distance within the copper slug, the heat flux to the engine wall can be calculated. The surface thermocouple is a fast-response thermocouple with a very thin sputtered junction on the surface of the slug.

\subsection{HANDLING}

This sensor should be handled with the same care that would be given to any other expensive and unique piece of instrumentation. It should be stored and packaged in padded containers when not in use. Special handling considerations are described below.

The surface thermocouple, as just stated, has a very thin sputtered junction. It is not considered fragile and has survived harsh environmental and wind tunnel testing. However it may be possible to damage this junction if care is not taken in handling and when installing or working around this gage. An accidental strike of a tool against the surface junction, for instance, or dropping something on the junction, might cause damage.

The shafts of the thermocouple leads extend back several inches from the copper slug. These shafts are to be bent as needed to fit inside the engine compartment and to route the lead wires. Until the installation, however, care should be taken not to bend these shafts.

\subsection{INSTALLATION}

The installation of the heat flux gage should be straightforward. The upper portion of the copper slug simply fits into the hole in the engine wall. Two 1/4-20 clearance holes are to be used to fasten the gage in place.

It will be necessary to bend the thermocouple lead shafts that extend from the back of the copper slug. This should be done carefully to ensure that the shafts are not crimped and the leads inside them are not damaged. The shafts may be bent however may be needed to fit within the engine compartment and route the lead wires.

The two type $\mathrm{T}$ thermocouples are to wired in series. The two constantan leads are to be spliced together and the two copper leads are to be acquired by the data system as a millivolt signal. The lead from the surface thermocouple should be the positive lead, and the lead from the backside thermocouple lead should be the negative lead. If this is not done, the only consequence will be that the signal will be inverted (opposite sign). This will be easy to diagnose and correct in the post-processing of the data.

The type $\mathrm{K}$ thermocouple in the backside of the slug is to be acquired by the data system as a type $\mathrm{K}$ thermocouple.

\subsection{FUNCTIONAL TESTING}

The best way to test the heat flux gage is to do a temperature jump test. Since the slug is copper, which has a high thermal conductivity, it is difficult to establish a large temperature difference between the surface and backside thermocouples. An easy way to 
do this is to abruptly place a heat source (such as a lighted match) near the surface thermocouple. This should produce a millivolt signal from the type $T$ thermocouples wired in series. The type $\mathrm{K}$ thermocouple should also start to change its reading as the heat passes through the copper slug. The signal from the type $T$ thermocouples will rapidly decrease again as the temperature in the copper slug becomes uniform. It is therefore necessary to monitor the output quickly as soon as the heat source is applied.

If the gage has not been installed in the engine yet, there is another way to establish a temperature difference in the gage that can be maintained. The backside of the copper slug can be placed in an ice or water bath. Then a heat source can be applied to the surface. This should produce a temperature difference that remains fairly constant. The output from the type $\mathrm{T}$ thermocouples should also remain constant during this test, as should the type $\mathrm{K}$ thermocouple reading.

\subsection{CLEANLINESS}

Cleanliness should not be a major factor for the heat flux gage. There are no gaps that need to be kept debris-free. The only possible concern is a coating of oil or grease that might cover the surface of the heat flux sensor. Such a coating could alter the heat flux into the surface of the sensor during flight. Therefore the surface of the heat flux sensor and the surrounding engine wall should be wiped down and generally kept clean of oil or grease. 
C.4 NASA Langley HXEM/AHSTF Skin Friction and Heat Flux Sensor Test Report 
NASA LANGLEY HXEM/AHSTF

SKIN FRICTION AND HEAT FLUX SENSOR

TEST REPORT

\title{
PERFORMED/PREPARED BY
}

\author{
TED SMITH \\ VIRGINIA TECH \\ AEROSPACE \& OCEAN ENGINEERING \\ 215 RANDOLPH HALL \\ BLACKSBURG, VA 24061
}

AUGUST 13, 2001 


\section{TABLE OF CONTENTS}

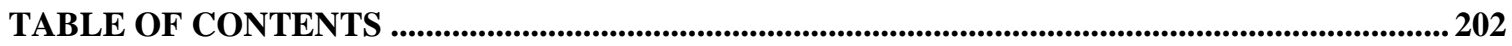

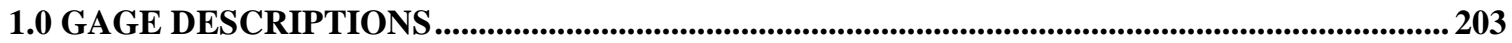

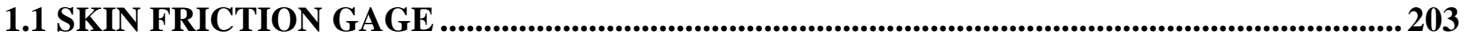

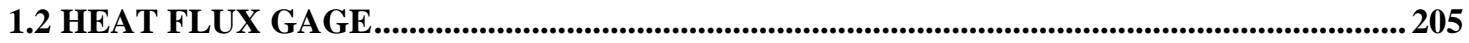

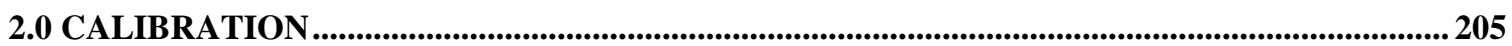

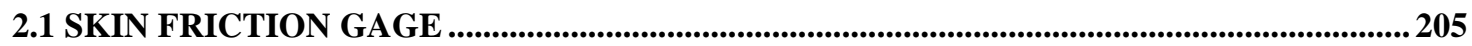

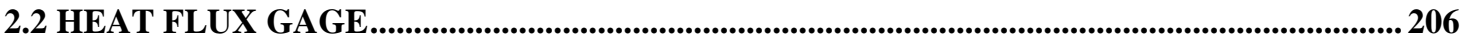

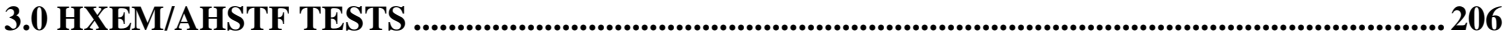

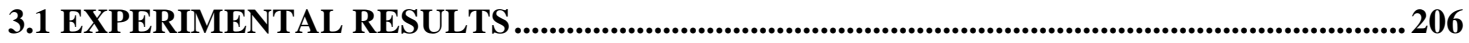

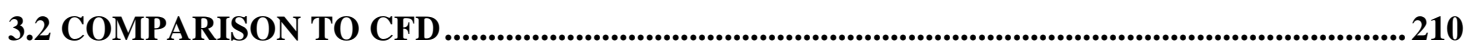




\subsection{GAGE DESCRIPTIONS}

\subsection{SKIN FRICTION GAGE}

The Virginia Tech skin friction sensor design consists of several components. The most important of the internal components is the flexure ring. This is the only moving part in the gage, and provides the mechanism by which the shear force is measured. It does this by deflecting under a load, and strain gages mounted on the flexures produce an output proportional to the load. The load is transferred to the flexure ring by means of a copper floating head that extends out of the brass housing, through the engine wall, and is flush with the flow surface of the engine. Shear forces acting on the wall also act on the surface of the floating head, causing it to deflect.

Figure 6 shows the internal components of the skin friction gage. These components are the flexure ring, a stainless steel adapter, the copper floating element, and a copper counterweight. The floating element is copper so that it matches the thermal properties of the engine wall. This is important in preventing a hot spot, where the heat flux is different than the rest of the wall. Since heat flux and skin friction are related, a different heat flux would imply an erroneous skin friction measurement. The stainless steel adapter acts as a passive thermal barrier because its thermal conductivity is low compared to that of copper. This slows the transfer of heat to the flexure ring, where the strain gages are located. The copper counterweight acts as an inertial damper, making the gage

a)

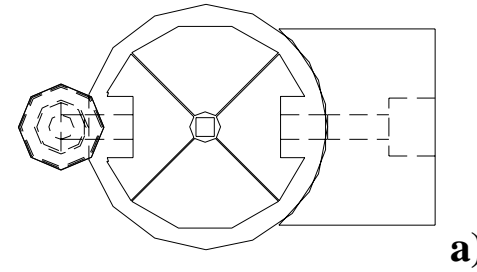

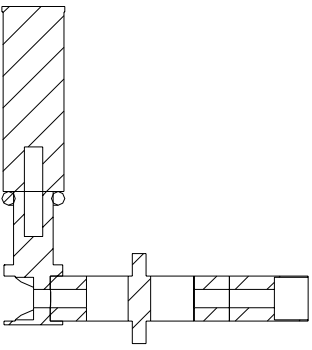

b)

c)

Figure 6: Skin Friction Gage Internal Components: a) Top Viesw b) Section View

c) Isometric View 

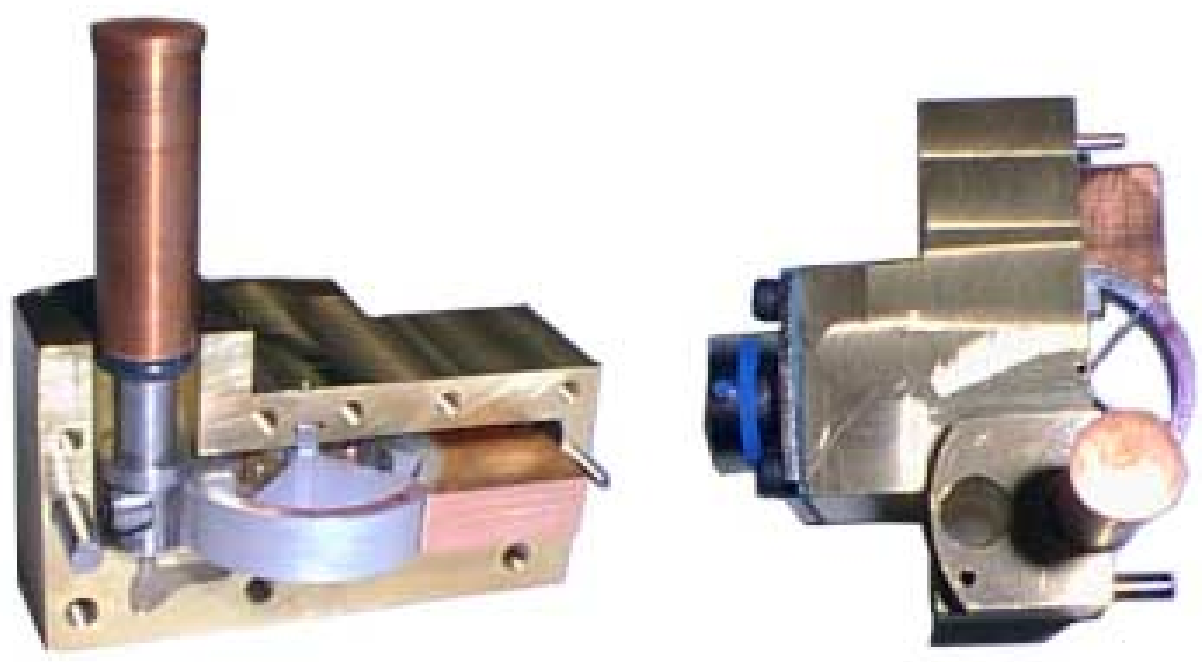

Figure 7: Internal Components Mounted in Partially Assembled Housing

insensitive to axial accelerations.

Figure 7 shows the internal components mounted in part of the two-piece housing, and Figure 8 shows the completely assembled gage. The housing is made of brass for two reasons. One is its low thermal conductivity (compared to copper), and the other is its ease of machining. The housing is very complicated, with many small, machined features. A flight-rated connector is fastened directly to the housing, and the leads from the strain gages are routed through it. A type $\mathrm{K}$ thermocouple is also built into the housing to monitor the temperature of the sensor. The leads from the thermocouple also pass through the connector.

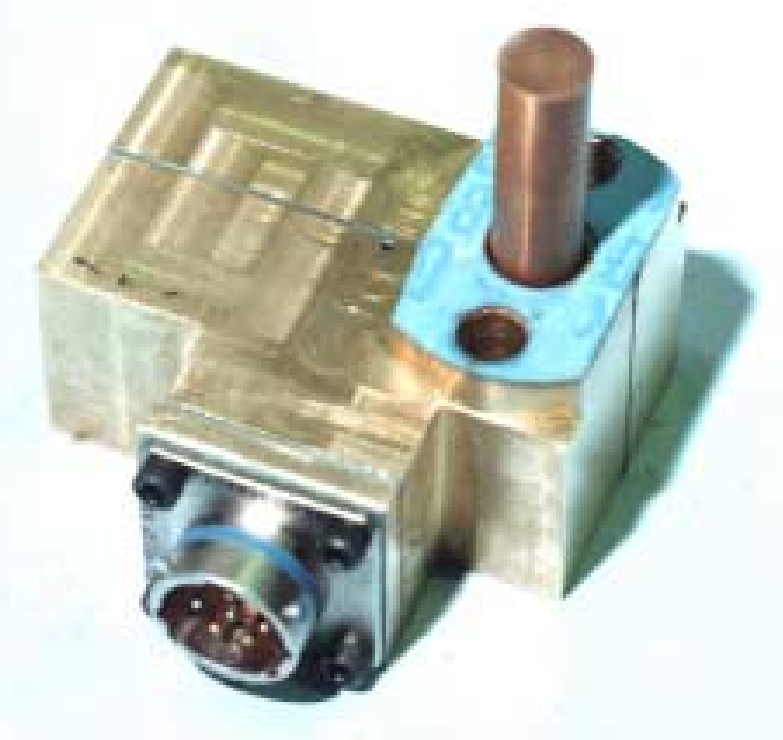

Figure 8: Assembled $C_{f}$ Gage 


\subsection{HEAT FLUX GAGE}

The heat flux gage is much smaller and simpler than the skin friction gage. It has no moving parts. It basically consists of a copper slug fitted with three thermocouples; one that mounts flush with the flow surface, and two that measure inside the slug. By measuring the temperature difference between the engine surface and a point at a known distance within the copper slug, the heat flux to the engine wall can be calculated. The surface thermocouple is a fast-response coaxial thermocouple with a very thin sputtered junction on the surface of the slug.

Since the engine wall is made of copper, type $\mathrm{T}$ thermocouples were chosen to measure the surface and backside temperature because they are made of copper and constantan leads. The coaxial thermocouple therefore has a copper sheath, with a very thin constantan wire in the center. This allows the coaxial thermocouple to closely match the thermal properties of the surrounding wall. Normally only two thermocouples would be required to get the temperature difference between the surface and the backside. However, the Hyper-X flight vehicle data system can only accept type K thermocouples. A type $\mathrm{K}$ thermocouple was therefore added adjacent to the type $\mathrm{T}$ thermocouple in the backside of the heat flux gage. The temperature measured by these two thermocouples should be the same. The two type $\mathrm{T}$ thermocouples are then wired in series by splicing the constantan leads together and acquiring the two copper leads as a millivolt signal. This millivolt signal corresponds to the temperature difference between the two type $\mathrm{T}$ thermocouples. By knowing the backside temperature from the type $\mathrm{K}$ thermocouple, the surface temperature can be found by using the voltage from the two type $T$ thermocouples, along with the calibration information for type $\mathrm{T}$ thermocouples.

\subsection{CALIBRATION}

\subsection{SKIN FRICTION GAGE}

The skin friction gage is calibrated with a direct force method. The gage is clamped horizontally, and weights are hung from the floating head. By multiplying the mass by the gravitational constant and then dividing by the surface area of the floating head, the voltage output from the sensor can be directly related to the shear on the floating head.

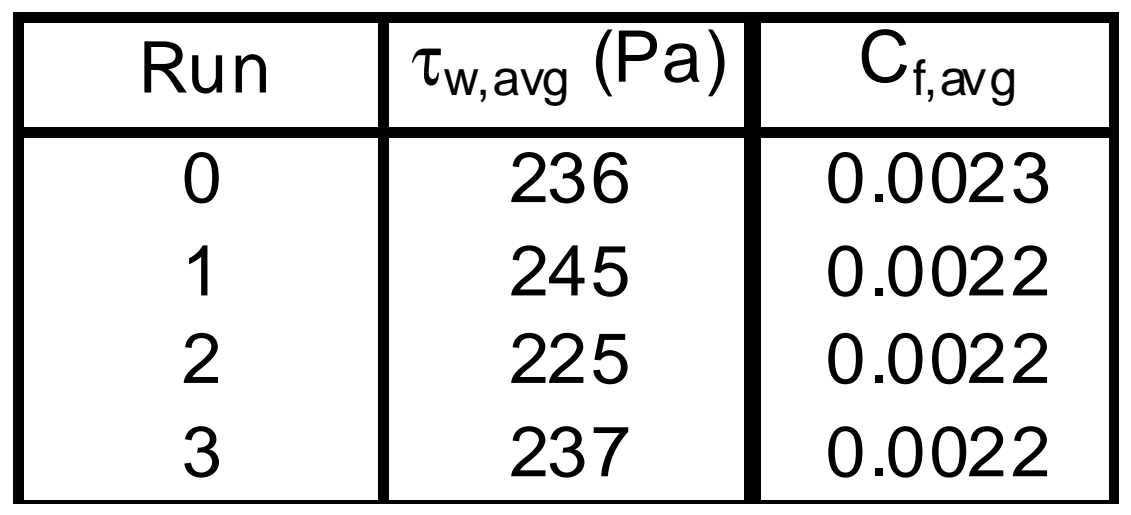

Table 1: Supersonic Wind Tunnel Results 


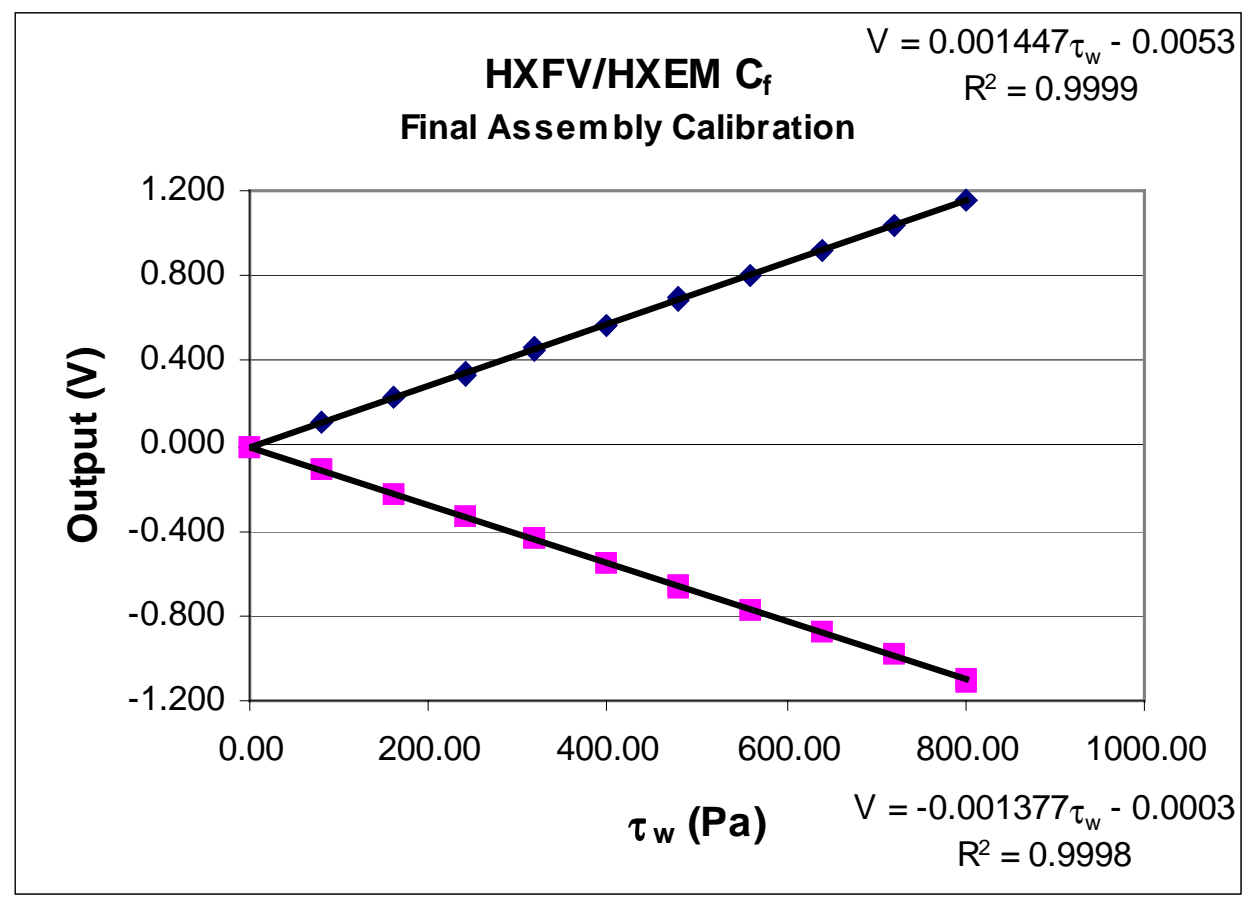

Figure 9: Sample Calibration Result

Figure 9 shows a sample calibration.

The skin friction gage was also tested in the Virginia Tech 9-inch by 9-inch supersonic wind tunnel. This wind tunnel provided a simple, cold Mach 2.4 flow with no pressure gradient. The skin friction gage output could then be compared to integral method estimates of skin friction coefficient. The results of these tests are shown in Table 1.

The results from the Virginia Tech supersonic wind tunnel were very steady and repeatable. The integral method estimated a $\mathrm{C}_{\mathrm{f}}$ of 0.0018 to 0.002 . The results from the skin friction gage were within roughly $10 \%$ of the estimate, giving confidence to the skin friction gage.

\subsection{HEAT FLUX GAGE}

The heat flux gage requires no calibration; instead it operates on first principles. A Class 3 numerical method will be used to estimate the heat flux into the gage. Using the time histories of the surface and backside temperatures and the known geometry and thermal properties of the gage, an unsteady heat conduction equation is iteratively solved until the difference between the measured and calculated temperature histories is minimized.

\subsection{HXEM/AHSTF TESTS}

\subsection{EXPERIMENTAL RESULTS}

The skin friction and heat flux gages were designed for testing on the Mach 10 Hyper-X flight vehicle. The best way to verify the gages prior to installation in the Hyper-X flight 
vehicle engine was to test in a wind tunnel model of the Hyper-X engine. This was done in the Hyper-X Engine Model (HXEM) in the NASA Langley Arc-Heated Scramjet Test Facility (AHSTF).

Testing of the skin friction and heat flux gages on the HXEM was a collaborative effort between Virginia Tech (graduate student Ted Smith and Prof. Joe Schetz) and NASA LaRC (Karen Cabell, Rick Irby) to demonstrate the operation and use of the Virginia Tech-designed gages in the AHSTF scramjet test environment.

AHSTF Test 133 was conducted on June 26 2001. Eight runs including two cold runs were conducted. One heat flux gage was installed on the 8-degree forebody (in place of heat flux gage HF05), and the skin friction gage was installed on the 11-degree forebody (in place of heat flux gage HF06). Each gage had two outputs. The heat flux gage had oune output for heat flux (temperature difference) and one output for backside temperature. The skin friction gage had one output for skin friction (shear level) and one output for internal gage temperature.

The engine was mounted at its nominal theta_model $=-3$ degrees orientation (angle between engine isolator axis and tunnel waterline, negative means leading edge down). The boundary layer trip strip on the 8-degree forebody was installed.

The tests were attempted Mach 6.5 runs (PT1 $\sim 550$ psia, HST $\sim 900 \mathrm{Btu} / \mathrm{lb}_{\mathrm{m}}$ ), except for tests 133.00 and 133.05, which were cold runs (no arc). The Mach 6 facility nozzle was installed. Data was acquired at $50 \mathrm{~Hz}$ by the Neff System and at $10 \mathrm{~Hz}$ by the PSI System. The skin friction signal was filtered at $1 \mathrm{~Hz}$ by the Neff, and the heat flux signal (temperature difference) was filtered at $10 \mathrm{~Hz}$.

The skin friction gage results of the AHSTF tests were very repeatable. Since the gage was not actively cooled (as previous skin friction sensors had been), there was thermal drift in the signal for each hot run. This drift was delayed until several seconds into the hot test period as the heat soaked through the engine wall. This allowed the skin friction level to be determined from the early portion of each test. Since the flow on the inlet was steady, any change in the signal during steady test segments could be attributed to thermal drift. This meant that the thermal drift could be subtracted out of the total skin friction gage signal. Figure 10 shows a sample result from one of the runs (all of the runs were very similar). The original signal, a polynomial fit to the thermal drift, and the corrected signal are shown.

Table 2 summarizes the results. The shear was normalized in two ways. The first was by dividing by the dynamic pressure of the facility nozzle. The second was by dividing by the local dynamic pressure. The local dynamic pressure was obtained from local static pressure measurements and an oblique shock calculation (for local Mach number). The flow from the facility nozzle was assumed to be vibrationally relaxed. The nozzle dynamic pressure was assumed to be constant between runs, while the local dynamic pressure varied slightly from run to run. 


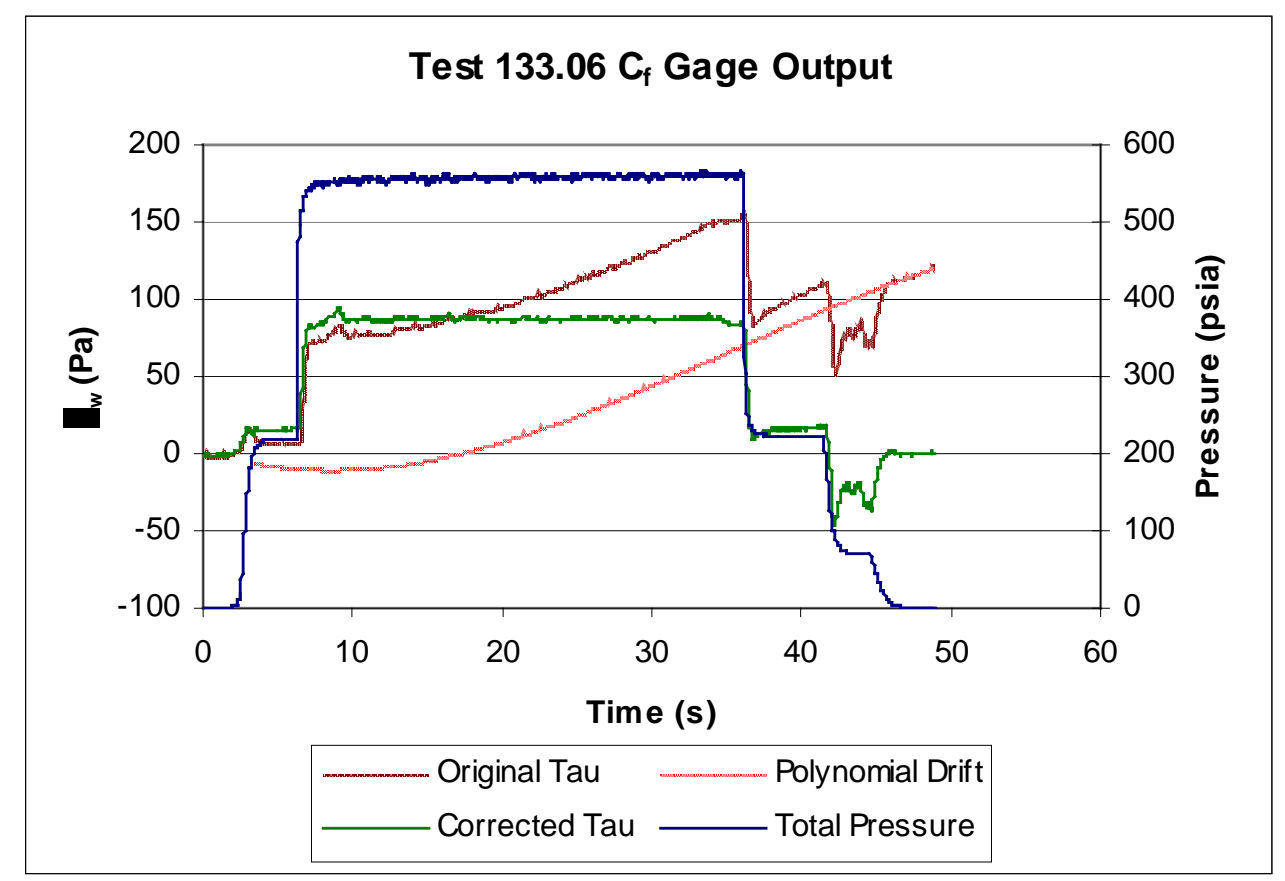

Figure 10: Sample AHSTF $C_{\mathrm{f}}$ Result

\begin{tabular}{|c|c|c|c|}
\hline Test & $\tau_{\mathrm{w}, \text { avg }}(\mathrm{Pa})$ & $\mathrm{C}_{\mathrm{f}, \text { an ozzle }}$ & $\mathrm{C}_{\mathrm{f}, \text { qlocal }}$ \\
\hline 133.01 & 74.9 & 0.0016 & 0.00094 \\
133.02 & 82.2 & 0.0017 & 0.00102 \\
133.03 & 87.3 & 0.0018 & 0.00108 \\
133.04 & 72.0 & 0.0015 & 0.00090 \\
133.06 & 87.3 & 0.0018 & 0.00108 \\
133.07 & 77.0 & 0.0016 & 0.00096 \\
\hline
\end{tabular}

Table 2: AHSTF $\mathrm{C}_{\mathrm{f}}$ Results Summary 


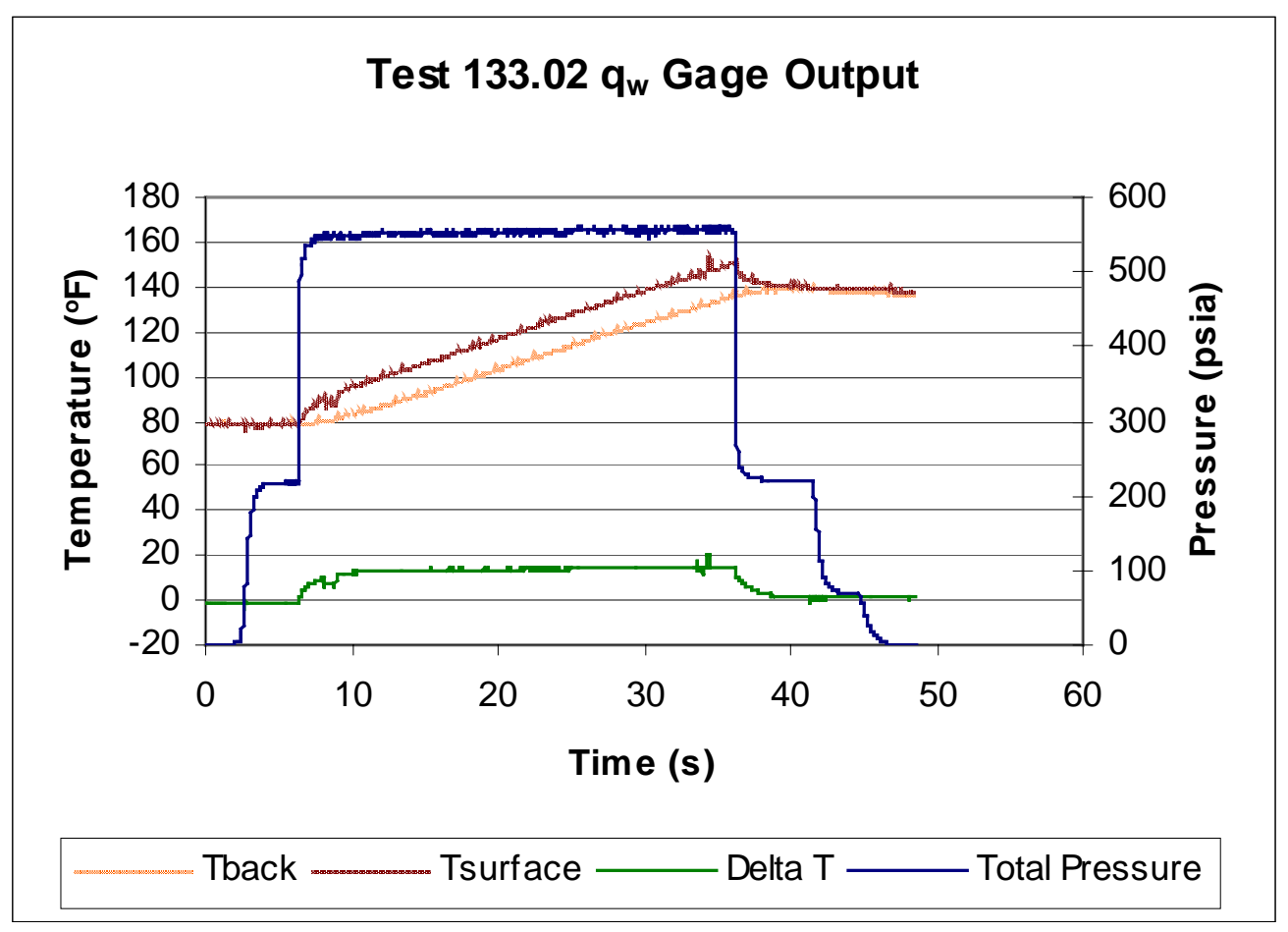

Figure 11: Sample AHSTF $q_{w}$ Result

The heat flux gage was also very repeatable. The temperature difference for each run reached a steady value of about $13^{\circ} \mathrm{R}$ between the surface and backside. Figure 11 shows a sample result. Table 3 summarizes the results. Since the temperature difference reached a steady value, it was possible to estimate the heat flux using the one dimensional relation $\mathrm{q}_{\mathrm{w}}=-\mathrm{k}(\mathrm{dT} / \mathrm{dy})$. A inverse method was also used to reduce the data and estimate the heat flux. Lastly, Trong Bui of NASA Dryden performed a CFD solution. The results of all three heat flux estimation methods are shown in Table 4. The negative values indicate heat transferred out of the fluid (and into the wall).

\begin{tabular}{|c|c|c|c|}
\cline { 2 - 4 } \multicolumn{1}{c|}{} & \multicolumn{3}{c|}{ Measurements } \\
\hline \multirow{2}{*}{ Run } & \multicolumn{2}{c|}{$\mathrm{T}_{\max }\left({ }^{\circ} \mathrm{F}\right)$} & Average \\
\cline { 2 - 3 } & Surface & Backside & $\Delta \mathrm{T}\left({ }^{\circ} \mathrm{F}\right)$ \\
\hline 133.01 & 88 & 79 & 13.1 \\
133.02 & 150 & 139 & 13.7 \\
133.03 & 218 & 208 & 13.1 \\
133.04 & 240 & 229 & 13.1 \\
133.06 & 264 & 253 & 13.2 \\
133.07 & 311 & 300 & 13.0 \\
\hline
\end{tabular}

Table 3: AHSTF Heat Flux Measurement Results 


\begin{tabular}{|c|c|c|c|c|c|c|}
\cline { 2 - 7 } \multicolumn{1}{c|}{} & \multicolumn{2}{c|}{ 1-D Estimate } & \multicolumn{2}{c|}{ Class 3 Estimate } & \multicolumn{2}{c|}{ CFD Estimate } \\
\cline { 2 - 7 } Run & \multicolumn{2}{c|}{ Average $\mathrm{q}_{\mathrm{w}}$} & \multicolumn{2}{c|}{ Average $\mathrm{q}_{\mathrm{w}}$} & \multicolumn{2}{c|}{ Average $\mathrm{q}_{\mathrm{w}}$} \\
\cline { 2 - 7 } & $\left(\mathrm{Btu} / \mathrm{ft}^{2} \mathrm{~s}\right)$ & $\left(\mathrm{W} / \mathrm{m}^{2}\right)$ & $\left(\mathrm{Btu} / \mathrm{ft}^{2} \mathrm{~s}\right)$ & $\left(\mathrm{W} / \mathrm{m}^{2}\right)$ & $\left(\mathrm{Btu} / \mathrm{ft}^{2} \mathrm{~s}\right)$ & $\left(\mathrm{W} / \mathrm{m}^{2}\right)$ \\
\hline 133.01 & -10.8 & -123100.3 & -9.16 & -104000 & $\mathrm{~N} / \mathrm{A}$ & $\mathrm{N} / \mathrm{A}$ \\
133.02 & -11.3 & -128738.5 & -8.79 & -99800 & $\mathrm{~N} / \mathrm{A}$ & $\mathrm{N} / \mathrm{A}$ \\
133.03 & -10.8 & -123100.3 & -9.13 & -103700 & $\mathrm{~N} / \mathrm{A}$ & $\mathrm{N} / \mathrm{A}$ \\
133.04 & -10.8 & -123100.3 & -9.10 & -103400 & $\mathrm{~N} / \mathrm{A}$ & $\mathrm{N} / \mathrm{A}$ \\
133.06 & -10.9 & -124040 & -9.17 & -104100 & $\mathrm{~N} / \mathrm{A}$ & $\mathrm{N} / \mathrm{A}$ \\
133.07 & -10.8 & -122160.6 & -8.83 & -100300 & -9.77 & -111000 \\
\hline
\end{tabular}

Table 4: AHSTF Heat Flux Estimates

\subsection{COMPARISON TO CFD}

To provide a way to evaluate the accuracy of the skin friction gage, a computational fluid dynamics (CFD) solution was performed for the AHSTF/HXEM test condition. The results from the experiment could then be compared to a numerical result.

The CFD solution had the following properties:

Code:

Dimensions:

Inflow Conditions:

Reference Inflow Conditions:

$\begin{array}{ll}\text { Ref. Mach: } & 5.869 \\ \text { Ref. Gamma: } & 1.399 \\ \text { Ref. Rgas: } & 288.15 \mathrm{~J} /(\mathrm{kf}-\mathrm{K}) \\ \text { Ref. Rho Static: } & 0.027055 \mathrm{~kg} / \mathrm{m}^{3} \\ \text { Ref. Rho*a }{ }^{2} & 2763.7 \mathrm{~Pa} \\ \text { Ref. Dyn. Pres.: } & 47598 \mathrm{~Pa} \\ \text { Ref. P Static: } & 1975.3 \mathrm{~Pa} \\ \text { Ref. T Static: } & 253.38 \mathrm{~K} \\ \text { Ref. a: } & 319.6 \mathrm{~m} / \mathrm{s} \\ \text { Ref. viscos.: } & 1.5365 \mathrm{E}-5 \mathrm{~N}^{*} \mathrm{~s}^{2} \mathrm{~m}^{2} \\ \text { Reynolds No.: } & 3.303 \mathrm{E} 6 \\ \text { Alpha (AoA): } & -3.0 \mathrm{deg} . \\ \text { U ref.: } & 1873 \mathrm{~m} / \mathrm{s} \\ \text { V ref.: } & -98.17 \mathrm{~m} / \mathrm{s}\end{array}$

Vulcan 4.2.0

2

Mach 6.5 Enthalpy

Finite Rate Calcultations Used Park 5 species/ 5 reactions air kinetics model

$\begin{array}{llll}\text { Species } & \text { Ref. Mass Fraction } & \text { Mol. Wt. } & \text { H0 }(299.14 \mathrm{~K})-\mathrm{H} 0(0 \mathrm{~K}) \\ \mathrm{N} 2 & 0.755338 & 28.0135 & 3.10727585 \mathrm{E} 5 \\ \mathrm{O} & 0.000000 & 15.9994 & 4.39848744 \mathrm{E} 5 \\ \mathrm{O} 2 & 0.221576 & 31.9988 & 2.76436210 \mathrm{E} 5 \\ \mathrm{~N} & 0.000000 & 14.0067 & 4.42433286 \mathrm{E} 5 \\ \mathrm{NO} & 0.023086 & 30.0061 & 3.13647573 \mathrm{E} 5\end{array}$


Freestream Turbulence Intensity:

Frozen or Finite Rate Equilibrium:

Gas Mixture:

Leading Edge Model:

Solution Starting Point:

Grid Dimensions:

Turbulence Model:

Wall Boundary Conditions:

Wall Temperature:

Range of $\mathrm{y}^{+}$Values at Wall:

Wall Functions Used:

Space Marching or Elliptic:

Convergence Criteria:
0.01

Both Were Conducted
Thermally Perfect
Blunt (0.030 inch radius)
Upstream of Leading Edge
353 (streamwise) by 161 (wall
normal)
Wall Function k- $\omega$ with
Compressibility Correction and
Laminar Block upstream to simulate
transition trip
Isothermal
407.462 Kelvin
0.25 to 6.8
Yes
Elliptic
L2 Norm Residuals Stop Decreasing

The CFD solution was performed using the conditions of test 133.07 at 20 seconds. The measurement at that time indicated a wall shear of about $77 \mathrm{~Pa}$. The CFD on the other hand, predicted a wall shear of $121 \mathrm{~Pa}$. There were, however, some items of note concerning the modeling of the flow for the CFD solution. There was an unexplained pressure drop observed during testing that was not part of the initial CFD solution. The turning angle of the engine wall in the CFD model was therefore modified. This allowed the CFD solution to reproduce the experimental pressure drop. However, the flow phenomenon that caused the pressure drop in the experiment is unknown and likely is not modeled in the CFD. Also, CFD is arguably poor at predicting complex turbulent boundary layer flows. An additional modeling concern is that of transition to turbulent flow. The CFD analysis simply assumed transition to occur abruptly at the transition strip. In reality, transition is likely to be a more complex process. Given these facts, the disagreement between the CFD and the measurement may be nothing more than the result of the CFD solving a problem that is different than the experimental case based on the modeling.

When the CFD is compared to the heat flux estimates, the results agree much closer than the skin friction results. The CFD solution predicted a wall heat flux of $-111000 \mathrm{~W} / \mathrm{m}^{2}$. The 1-D method estimated heat flux of $-122000 \mathrm{~W} / \mathrm{m}^{2}$, while the inverse method estimated $-100000 \mathrm{~W} / \mathrm{m}^{2}$. The $1-\mathrm{D}$ method likely over-predicts the heat flux due to material properties and lateral convection that occur in the actual physical case. Therefore, the difference between the CFD and the inverse method is roughly $10 \%$. 


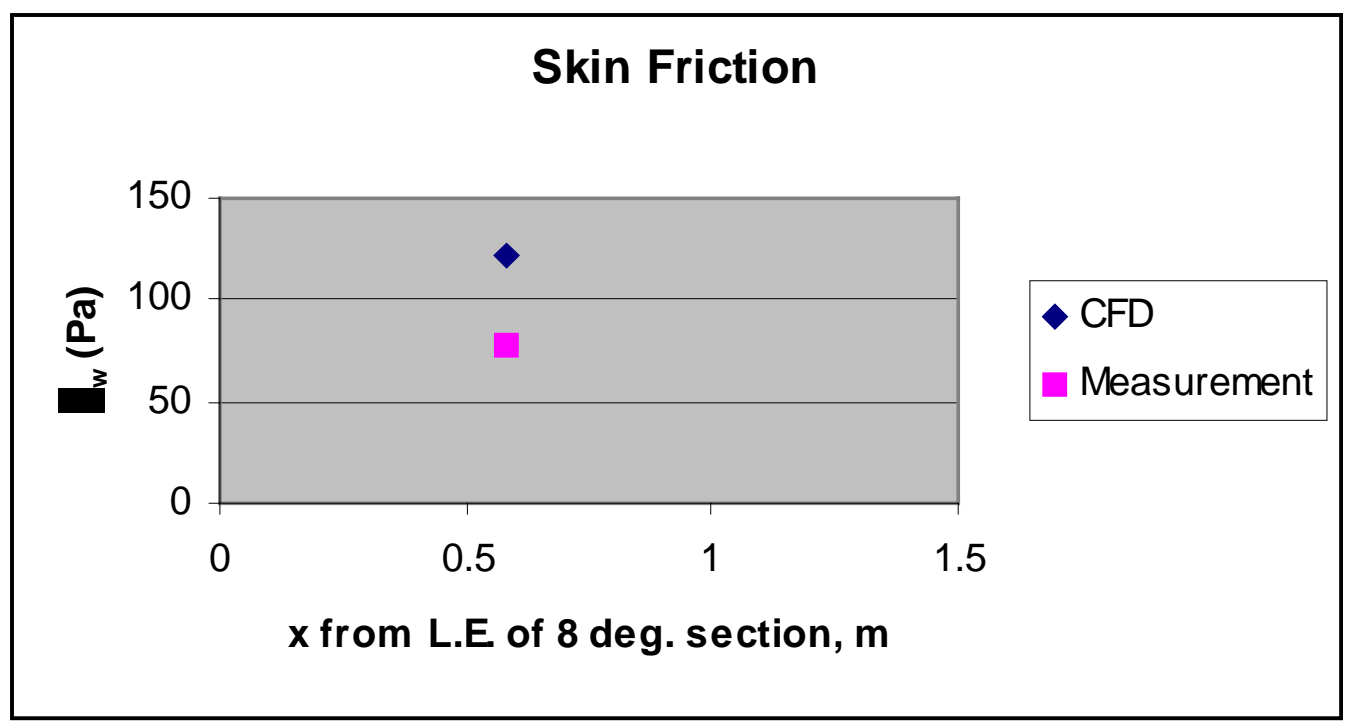

Figure 12: Wall Shear Comparison

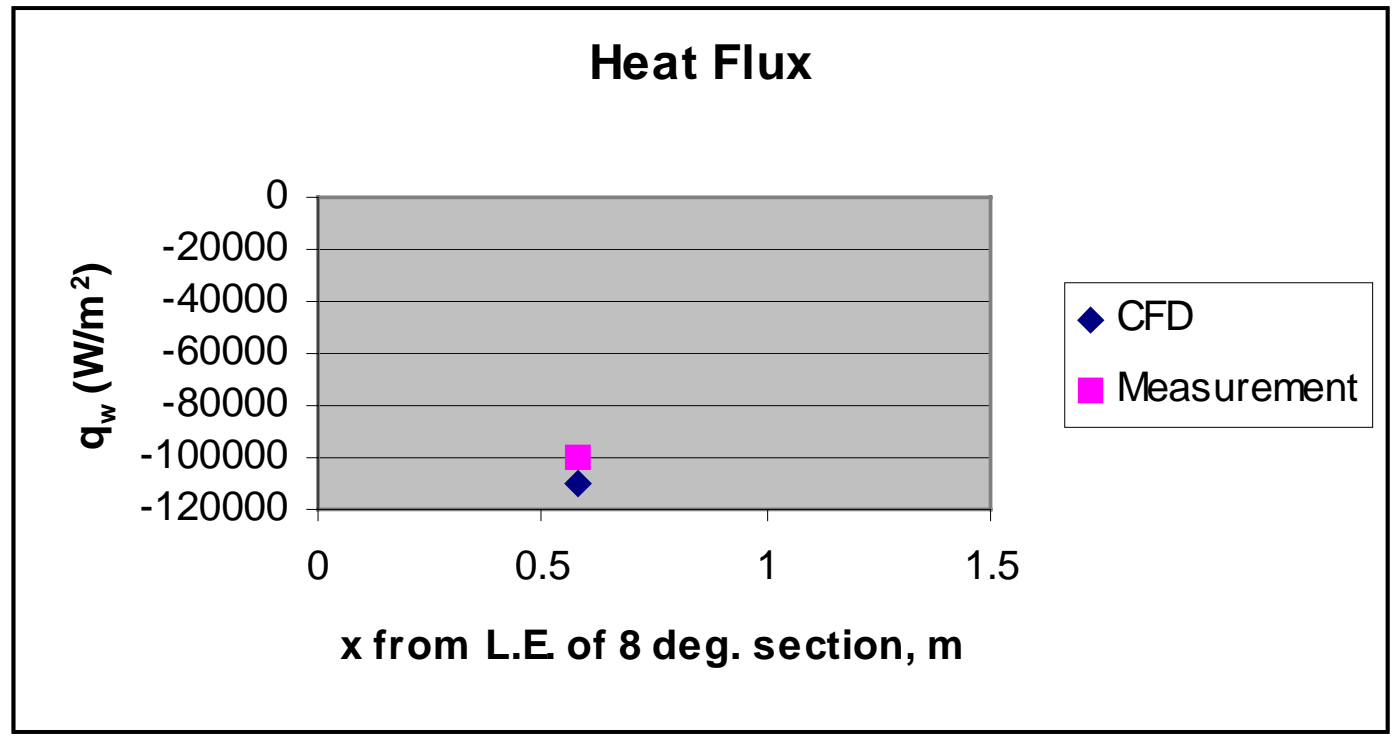

Figure 13: Heat Flux Comparison 


\section{VITA}

Theodore Brooke Smith was born the youngest of three children to Dean Richard and Nancy Irene Smith on August 16, 1975 in Beaver Falls, Pennsylvania. Two sisters, Kimberley and Leslie, preceded him. He attended Beaver County Christian School, where he graduated valedictorian in 1994. Subsequently, he obtained a Bachelor of Science in Engineering with a Mechanical Engineering concentration from Geneva College in May 1998. Upon graduation from Geneva, he entered the Aerospace \& Ocean Engineering Department at Virginia Polytechnic Institute and State University in pursuit of a Ph.D.

On July 3, 1998, Theodore Brooke Smith was married to Julie Ann Keller near Pittsburgh, Pennsylvania. Upon completing his studies at Virginia Tech in Fall 2001, Theodore and Julie moved to Manassas, Virginia, and Theodore began employment as a Senior Test Engineer at Atlantic Research Corporation, where he worked with ramjet engines.

Theodore B. Smith's other interests include church activities, running, reading, and various other outdoor activities.

Theodore B. Smith

September 2001

Blacksburg, Virginia 